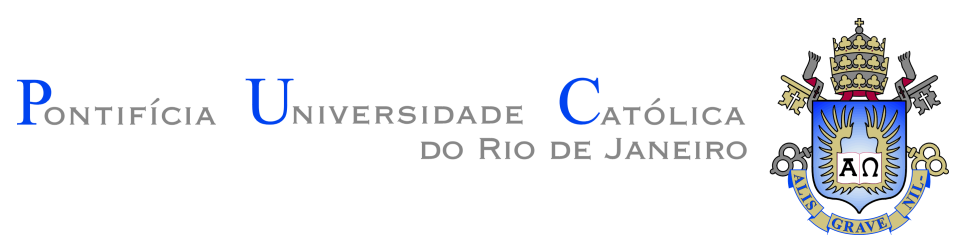

Camila Sobrinho Crispim

\title{
Dinâmicas minimais em conjuntos de Cantor e diagramas de Bratteli
}

Dissertação de Mestrado

Dissertação apresentada como requisito parcial para obtenção do grau de Mestre pelo Programa de Pós-graduação em Matemática Pura, do Departamento de Matemática da PUC-Rio.

Orientador: Prof. Lorenzo Justiniano Díaz Casado 


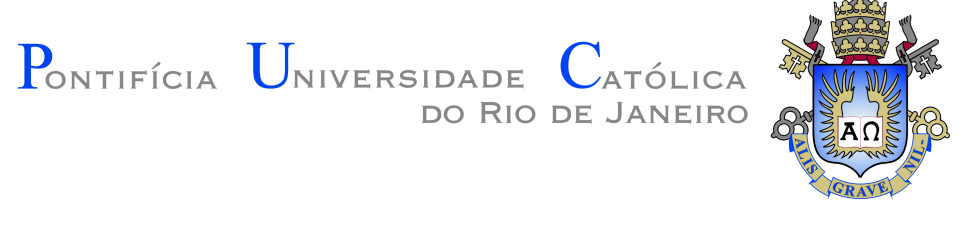

Camila Sobrinho Crispim

\section{Dinâmicas minimais em conjuntos de Cantor e diagramas de Bratteli}

Dissertação apresentada como requisito parcial para obtenção do grau de Mestre pelo Programa de Pós-graduação em Matemática Pura da PUC-Rio. Aprovada pela Comissão Examinadora abaixo:

Prof. Lorenzo Justiniano Díaz Casado Orientador Departamento de Matemática - PUC-Rio

Prof. Rafael Oswaldo Ruggiero Rodriguez Departamento de Matemática - PUC-Rio

Prof. Carlos Meniño Cotón Departamento de Análise - UFF

Prof. Ali Messaoudi

Departamento de Matemática - UNESP

Prof. Marcelo Sobottka Departamento de Matemática - UFSC 
Todos os direitos reservados. A reprodução, total ou parcial do trabalho, é proibida sem a autorização da universidade, do autor e do orientador.

\section{Camila Sobrinho Crispim}

Bacharel em Matemática pela Universidade Federal Fluminense (UFF) em 2018.

Ficha Catalográfica

Sobrinho Crispim, Camila

Dinâmicas minimais em conjuntos de Cantor e diagramas de Bratteli / Camila Sobrinho Crispim; orientador: Lorenzo Justiniano Díaz Casado. - 2021.

184 f: il. color. ; $30 \mathrm{~cm}$

Dissertação (mestrado) - Pontifícia Universidade Católica do Rio de Janeiro, Departamento de Matemática, 2021.

Inclui bibliografia

1. Matemática - Teses. 2. Sistemas Dinâmicos - Teses. 3. Conjunto de Cantor. 4. Diagrama de Bratteli. 5. Equivalência orbital. 6. Grupo abeliano ordenado. 7. Minimalidade. 8. Odômetro. I. Diaz, L.J.. II. Pontifícia Universidade Católica do Rio de Janeiro. Departamento de Matemática. III. Título. 


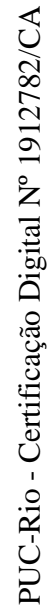

Aos meus pais. 


\section{Agradecimentos}

Agradeço aos meus pais por todo o suporte, carinho, dedicação e apoio em todas as fases da minha vida.

Ao meu orientador Lorenzo Díaz, por todos os ensinamentos, pelas diversas conversas com entusiasmo e paciência que foram de fundamental importância para o desenvolvimento deste trabalho.

À Maryam Hosseini pelas contribuições fornecidas ao último capítulo deste trabalho.

Aos professores Ali Messaoudi, Carlos Meniño Cotón, Marcelo Sobottka e Rafael Oswaldo Ruggiero por aceitarem fazer parte da banca.

À minha orientadora de iniciação científica, a professora Cybele Vinagre, por ter me incentivado e por ser uma amiga.

Ao Luiz pelo apoio e companheirismo durante todos esses anos e por sempre me incentivar a dar o meu melhor.

Aos meus amigos, em especial: Giovanna, Isabela, Guilherme e Raphael, que me acompanham desde a graduação.

Ao professor Bruno Santiago por ter me apresentado Sistemas Dinâmicos durante o curso de Equações Diferenciais Ordinárias na graduação.

À Capes, à Petrobras e à PUC-Rio pelo apoio financeiro.

O Presente trabalho foi realizado com apoio da Coordenação de Aperfeiçoamento de Pessoal de Nível Superior-Brasil (Capes) - Código de financiamento 001. 


\section{Resumo}

Sobrinho Crispim, Camila; Diaz, L.J.. Dinâmicas minimais em conjuntos de Cantor e diagramas de Bratteli. Rio de Janeiro, 2021. 184p. Dissertação de Mestrado - Departamento de Matemática, Pontifícia Universidade Católica do Rio de Janeiro.

Um diagrama de Bratteli $\mathfrak{B}$ é um objeto combinatório representado por um grafo dividido em infinitos níveis, cada um com número finito de vértices e de arestas entre vértices de níveis consecutivos. Além disso, todo vértice possui ligação com vértices dos níveis precedente e sucessor.

Estudamos, do ponto de vista topológico, o espaço dos caminhos infinitos formados pelas arestas de um diagrama de Bratteli, denotado por $X_{\mathfrak{B}}$. Estabelecemos uma relação de equivalência neste espaço, denominada AF. Quando é possível definir uma ordem parcial em $X_{\mathfrak{B}}$ o diagrama é dito ordenado; neste caso, definimos um homeomorfismo em $X_{\mathfrak{B}}$ denominado de função de Bratteli-Vershik. Consideramos sistemas dinâmicos minimais definidos em conjuntos de Cantor e associamos a estes diagramas de Bratteli ordenados.

Um exemplo paradigmático de um conjunto de Cantor é o espaço das sequências infinitas formadas por $0^{\prime} s$ e $1^{\prime} s$, munido de uma métrica apropriada. Neste espaço são definidas as funções odômetro. Definimos a relação de equivalência orbital, na qual duas sequências são equivalentes se estão na mesma órbita do odômetro, e a relação de equivalência de caudas, onde duas sequências são equivalentes se a partir de alguma entrada elas são iguais. Estudamos como estas duas relações estão relacionadas. Provamos que o odômetro diádico é um homeomorfismo minimal definido em um conjunto de Cantor e, portanto, pode ser associado a um diagrama de Bratteli ordenado.

Uma relação de equivalência é dita étale quando admite uma topologia gerada por uma ação local. Dois exemplos são as relações AF e orbital. Dada uma relação de equivalência étale $R$ em um espaço $X$, definimos um invariante algébrico $D(X, R)$. Construímos o grupo de dimensão de um diagrama de Bratteli. Provamos, então, que dado um diagrama de Bratteli $\mathfrak{B}$, seu grupo de dimensão é isomorfo a $D\left(X_{\mathfrak{B}}, R_{\mathfrak{B}}\right)$, onde $R_{\mathfrak{B}}$ é relação $\mathrm{AF}$ de $\mathfrak{B}$. Finalmente, estudamos sob quais condições um grupo abeliano ordenado é o grupo de dimensão de um diagrama de Bratteli.

Esta dissertação é baseada no livro de Ian F. Putnam "Cantor minimal systems", publicado em University Lecture Series, 70. American Mathematical Society, Providence, RI, 2018. [6]

\section{Palavras-chave}

Conjunto de Cantor; Diagrama de Bratteli; Equivalência orbital; Grupo abeliano ordenado; Minimalidade; Odômetro. 


\section{Abstract}

Sobrinho Crispim, Camila; Diaz, L.J. (Advisor). Minimal dynamics on Cantor sets and Bratteli diagrams. Rio de Janeiro, 2021. 184p. Dissertação de Mestrado - Departamento de Matemática, Pontifícia Universidade Católica do Rio de Janeiro.

A Bratteli diagram $\mathfrak{B}$ is a combinatorial object represented by a graph divided into infinite levels, each level with a finite number of vertices and edges between vertices of consecutive levels. Moreover, every vertex is connected to vertices of the preceding and successor levels.

We study, from a topological point of view, the space of infinite paths formed by the edges of a Bratteli diagram, denoted by $X_{\mathfrak{B}}$. We establish an equivalence relation on this space, called the AF relation. When it is possible to define a partial order in $X_{\mathfrak{B}}$ the Bratteli diagram is called ordered; in this case, we define a homeomorphism on $X_{\mathfrak{B}}$ called the Bratteli-Vershik function. We consider minimal dynamic systems defined on Cantor sets and associate to these systems ordered Bratteli diagrams.

A paradigmatic example of a Cantor set is the space of the infinite sequences formed by $0^{\prime} s$ and $1^{\prime} s$, equipped with an appropriate metric. In this space, are defined the odometer functions. We define the orbital equivalence relation, in which two elements of the Cantor set are equivalent if they are in the same orbit of the odometer, and the tail equivalence relation, where two sequences are equivalents if they differ in only finitely many entries. We study how these equivalence relations are related. We prove that the dyadic odometer is a minimal homeomorphism and, therefore, it can be associated to a ordered Bratteli diagram.

An equivalence relation is called étale if it admits a topology generated by a local action. Two examples are the AF equivalence relation and the orbital equivalence relation above. Given an étale equivalence relation $R$ on a space $X$ we define an algebraic invariant $D(X, R)$. We construct the dimension group of a Bratteli diagram. Then, we prove that given a Bratteli diagram $\mathfrak{B}$, its dimension group is isomorphic to $D\left(X_{\mathfrak{B}}, R_{\mathfrak{B}}\right)$, where $R_{\mathfrak{B}}$ is the AF equivalence relation of $\mathfrak{B}$. Finally, we study under which conditions an ordered abelian group is the dimension group for some Bratteli diagram.

This master thesis is based on the book by Ian F. Putnam "Cantor minimal systems", published in University Lecture Series, 70. American Mathematical Society, Providence, RI, 2018. [6]

\section{Keywords}

Abelian ordered group; Bratteli diagram; Cantor set; Minimality; odometer. 


\section{Sumário}

1 Introdução 11

1.1 Introdução 11

1.1.1 O odômetro diádico e diagrama de Bratteli 12

1.1.2 Diagramas de Bratteli e sistemas de Cantor minimais $\quad 15$

1.2 Organização desta dissertação 20

2 Odômetro $\quad 23$

2.1 Adição de sequências 23

2.2 Odômetro 23

2.3 Outra relação de equivalência 30

3 Conjuntos de Cantor $\quad 33$

3.1 Conceitos preliminares 33

3.2 Conjuntos de Cantor 41

4 Equivalência orbital 45

5 Diagrama de Bratteli $\quad 48$

5.1 Diagrama de Bratteli: definição e compacidade do espaço $X_{\mathfrak{B}} \quad 48$

5.2 Propriedade telescópica de um diagrama de Bratteli 55

5.3 Relação de equivalência AF 56

$\begin{array}{lll}5.4 & \text { Exemplos } & 59\end{array}$

$\begin{array}{lll}6 & \text { O modelo de Bratteli-Vershik } & 61\end{array}$

$\begin{array}{lll}6.1 & \text { Diagrama de Bratteli ordenado } & 61\end{array}$

6.2 As relações $R_{\varphi_{\mathfrak{B}}}$ e $R_{\mathfrak{B}} \quad 66$

7 Partições de Kakutani-Rokhlin $\quad 72$

$\begin{array}{lll}7.1 \text { Castelos } & 72\end{array}$

$\begin{array}{lll}7.1 .1 & \text { Construção de } \mathfrak{B} & 78\end{array}$

$\begin{array}{lll}\text { 7.1.2 Construção da conjugação entre } X_{\mathfrak{B}} \text { e } X & 79\end{array}$

8 Relações de equivalência étale $\quad 84$

8.1 Ações locais e relações de equivalência Étale 84

$8.2 R_{\mathfrak{B}}$ como uma relação de equivalência étale 88

8.2.1 Relação $A F$ em um conjunto de Cantor 92

8.3 $R_{\varphi}$ como uma relação de equivalência étale 97

9 Conceitos de Álgebra $\quad 100$

$\begin{array}{lll}9.1 & \text { Grupos } & 100\end{array}$

$\begin{array}{lll}9.2 & \text { Grupos abelianos ordenados } & 101\end{array}$

$\begin{array}{ll}10 \text { O invariante D } & 104\end{array}$

$\begin{array}{lll}10.1 \text { O grupo } C(X, \mathbb{Z}) & 104\end{array}$

$\begin{array}{lll}10.2 \text { O invariante } & 107\end{array}$ 
11 O grupo de dimensão de um diagrama de Bratteli

11.1 Limite indutivo de grupos

11.2 O grupo de dimensão de um diagrama de Bratteli 119

11.2.1 A função $h_{\mathfrak{B}_{n}} \quad 119$

11.2.2 O grupo de dimensão de um diagrama de Bratteli 122

$\begin{array}{ll}\text { 11.2.3 O grupo de dimensão e a propriedade telescópica } & 127\end{array}$

$\begin{array}{ll}\text { 11.2.4 O grupo de dimensão e a ordem unitária } & 128\end{array}$

$12 \mathrm{O}$ invariante para as relações AF e orbital 133

12.1 O invariante para relações-AF 133

12.2 O invariante para o modelo de Bratteli-Vershik 138

13 O Teorema de Effros-Handelman-Shen 144

13.1 O Teorema de Effros-Handelman-Shen 144

$\begin{array}{ll}\text { 13.1.1 O Teorema de Shen } & 147\end{array}$

13.1.2 Fim da prova do Teorema de Effros-Handelman-Shen 156

14 O Teorema de Bratteli-Elliot-Krieger $\quad 160$

14.1 Teorema de Bratteli-Elliot-Krieger 160

$\begin{array}{lll}15 & \text { Equivalência orbital forte } & 167\end{array}$

$\begin{array}{ll}15.1 \text { Cociclos orbitais } & 167\end{array}$

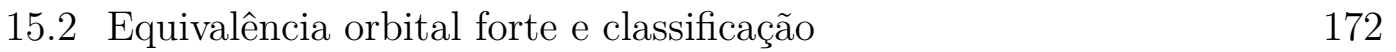

16 Equivalência orbital versus conjugação 181

$\begin{array}{ll}16.1 \text { Equivalência orbital versus conjugação } & 181\end{array}$

$\begin{array}{lr}\text { Referências bibliográficas } & 184\end{array}$ 


\section{Lista de figuras}

Figura 1.1 Uma rotação em $\mathbf{S}^{1} \quad 11$

$\begin{array}{lll}\text { Figura } 1.2 & \text { Grafo infinito } \mathfrak{B} . & 13\end{array}$

Figura 1.3 Diagrama de Bratteli que não é próprio 16

Figura 2.1 Ilustração da permutação dos cilindros de tamanho dois. 29

Figura 3.1 Ilustração de uma sequência de partições refinadoras. 36

Figura 5.1 Exemplo de um diagrama de Bratteli 48

Figura 5.2 Diagrama de Bratteli que representa $\{0,1\}^{\mathbb{N}} \quad 60$

Figura 5.3 Diagrama $\mathfrak{T}$, onde $X_{\mathfrak{T}}$ é um subconjunto de $\{0,1\}^{\mathbb{N}} . \quad 60$

Figura 6.1 Diagrama de Bratteli próprio. $\quad 65$

$\begin{array}{lll}\text { Figura 6.2 Diagrama de Bratteli } \mathfrak{T} & 65\end{array}$

$\begin{array}{lll}\text { Figura 6.3 Diagrama de Bratteli } \mathfrak{Q} & 66\end{array}$

$\begin{array}{lll}\text { Figura 7.1 Ilustração de um castelo. } & 75\end{array}$

Figura 11.1 Diagrama de Bratteli $\mathfrak{B} \quad 122$

Figura 11.2 Diagrama de Bratteli $\mathfrak{T}=(W, F, r, s) \quad 124$

Figura 11.3 Exemplo de diagrama de Bratteli com mais de um elemento em $V_{0}$. 129

Figura 13.1 Diagrama comutativo $\quad 155$

Figura 13.2 Diagrama comutativo 157

Figura 14.1 Diagrama de Bratteli $\mathfrak{E} . \quad 161$

Figura $14.2 \mathfrak{Q}(\mathfrak{B}, \mathfrak{T}) . \quad 162$

Figura 16.1 Primeiro passo da primeira etapa da Construção da transformação de Chacón.[1] 182

Figura 16.2 Segundo passo da primeira etapa da construção da transformação de Chacón.[1] 183

Figura 16.3 Fim da primeira etapa da construção da transformação de Chacón. [1] 183 


\section{Introdução}

\section{1}

\section{Introdução}

Na teoria dos sistemas dinâmicos um dos objetos centrais de estudo é o comportamento das órbitas. Isto é, dados um espaço métrico $(X, d)$, um homeomorfismo $\varphi: X \rightarrow X$ e um ponto $x$ de $X$, estudamos como se comporta o conjunto

$$
\mathcal{O}_{\varphi}(x):=\left\{\varphi^{n}(x) \mid n \in \mathbb{Z}\right\},
$$

denominado de órbita de $x$ por $\varphi$. Associada à função $\varphi: X \rightarrow X$ definimos a relação de equivalência orbital, denotada por $R_{\varphi}$, onde $x, y \in X$ são equivalentes se, e só se, existe $n \in \mathbb{Z}$ tal que $y=\varphi^{n}(x)$. A classe de equivalência de um ponto $x$ na relação orbital é o conjunto $\mathcal{O}_{\varphi}(x)$.

O homeomorfismo $\varphi: X \rightarrow X$ é dito minimal se para todo $x \in X$ o conjunto $\mathcal{O}_{\varphi}(x)$ é denso. Baseado nesta definição, dizemos que uma relação de equivalência definida no conjunto $X$ é minimal se todas suas classes de equivalência são densas. Dessa forma, a função $\varphi$ é minimal se e somente se a relação $R_{\varphi}$ é minimal.

Um exemplo muito simples de função minimal é a rotação no círculo

$$
\mathbf{S}^{1}:=\left\{e^{2 \pi i \theta} \mid 0 \leq \theta<2 \pi\right\},
$$

por um ângulo irracional $\alpha$, definida por

$$
\rho_{\alpha}\left(e^{2 \pi i \theta}\right)=e^{2 \pi i(\theta+\alpha)} .
$$

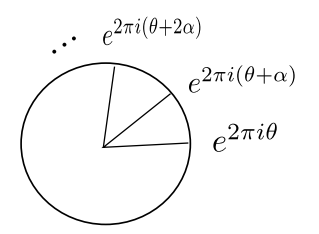

Figura 1.1: Uma rotação em $\mathbf{S}^{1}$ 
Como na rotação deslocamos sempre o mesmo ângulo, observamos que as rotações no círculo são isometrias.

O foco desta dissertação é estudar sistemas dinâmicos $(X, \varphi)$ tais que $X$ é um conjunto de Cantor e a função $\varphi$ é um homeomorfismo minimal.

Um conjunto de Cantor é um espaço métrico compacto, totalmente desconexo e sem pontos isolados. Veremos que todos os conjunto de Cantor são homeomorfos.

A partir de agora, consideraremos que $X$ é um conjunto de Cantor e $\varphi: X \rightarrow X$ um homeomorfismo. Veremos, em primeiro lugar, a maioria dos conceitos apresentados nesta dissertação para o exemplo mais simples e paradigmático de dinâmicas minimais em um conjunto Cantor, que generaliza as rotações irracionais: a função odômetro diádico.

\subsection{1}

\section{O odômetro diádico e diagrama de Bratteli}

Considere o espaço das sequências infinitas formadas por $0^{\prime} s$ e $1^{\prime} s$, denotado por $\{0,1\}^{\mathbb{N}}$. Denotamos um elemento de $\{0,1\}^{\mathbb{N}}$ por $\mathbf{x}=\left(x_{1} x_{2} \ldots\right)=$ $\left(x_{n}\right)_{n \geq 1}$, onde $x_{i} \in\{0,1\}$ para todo $i \geq 1$. Em particular, denotamos $\mathbf{0}=(00 \ldots)$ e $\mathbf{1}=(11 \ldots)$. Consideramos para $\{0,1\}^{\mathbf{N}}$ a métrica

$$
d(\mathbf{x}, \mathbf{y})=\left\{\begin{array}{l}
1, \quad \text { se } x_{1} \neq y_{1}, \\
\inf \left\{2^{-n} \mid n \geq 1, x_{i}=y_{i}, \text { para todo } 1 \leq i \leq n\right\}, \quad \text { se } x_{1}=y_{1}
\end{array}\right.
$$

Desta forma, o espaço métrico $\left(\{0,1\}^{\mathbb{N}}, d\right)$ é um conjunto de Cantor.

O odômetro diádico é a função definida por:

$$
\Phi:\{0,1\}^{\mathbf{N}} \rightarrow\{0,1\}^{\mathbf{N}}, \quad \Phi(\mathbf{x})=\mathbf{x}+(1000 \ldots)
$$

precisamos definir o que é somar o elemento $(1000 \ldots)$ a um elemento de $\{0,1\}^{\mathbb{N}}$. Primeiro, definimos que

$$
\mathbf{1}+(1000 \ldots)=\mathbf{0}
$$

Agora, considere $\mathbf{x}=\left(x_{1} x_{2} \ldots\right) \neq \mathbf{1}$, existe $n$ mínimo tal que $x_{n}=0$, definimos, então,

$$
\mathbf{x}+(1000 \ldots)=\left(00 \ldots 01 x_{n+1} x_{n+2} \ldots\right)
$$

Repare que, assim como nas rotações irracionais, no odômetro estamos sempre transladando um mesmo valor, portanto, o odômetro também é uma isometria.

Provamos que o odômetro é um homeomorfismo e que a relação de equivalência $R_{\Phi}$ é minimal. Portanto o odômetro diádico é um homeomorfismo minimal definido em um conjunto de Cantor. 


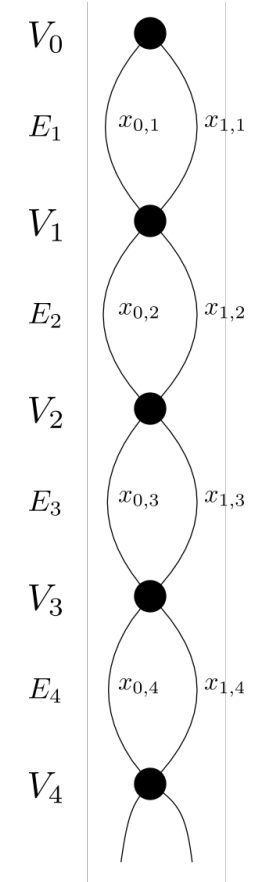

Figura 1.2: Grafo infinito $\mathfrak{B}$.

Considere o grafo infinito apresentado na Figura 1.2, este diagrama é um caso particular de diagrama de Bratteli. Note que $\mathfrak{B}$ está dividido em infinitos níveis de vértices $\left\{V_{n}\right\}_{n \geq 0}$, onde $V_{n}=\left\{v_{n}\right\}$ e de arestas $\left\{E_{n}\right\}_{n \geq 1}$, onde $E_{n}=\left\{x_{0, n}, x_{1, n}\right\}$. Repare, também, que não há nenhum vértice que não receba uma aresta. Assim, existem "caminhos infinitos" formados pelas arestas do grafo. Denotamos o espaço de todos os caminhos infinitos formado pelas arestas de $\mathfrak{B}$ por $X_{\mathfrak{B}}$ e um elemento de $X_{\mathfrak{B}}$ por $\mathbf{x}=\left(x_{i}\right)_{i \geq 1}$, onde $x_{i} \in\left\{x_{0, i}, x_{1, i}\right\}$ para todo $i \geq 1$. O espaço $X_{\mathfrak{B}}$ é compacto quando considerada uma métrica apropriada. Repare que como há uma escolha diádica para as entradas do caminho, o espaço $X_{\mathfrak{B}}$ é o mesmo que $\{0,1\}^{\mathbb{N}}$.

Definimos uma relação de equivalência em $X_{\mathfrak{B}}$, denominada relação $A F$ e denotada por $R_{\mathfrak{B}}$, onde dois elementos são equivalentes se diferem em um número finito de entradas. Podemos definir, para cada $n \geq 1$,

$$
x_{\min , n}:=x_{0, n}<x_{1, n}=: x_{\max , n} .
$$

Denominamos $x_{\min , n}$ e $x_{\max , n}$ como, respectivamente, as arestas mínima e máxima no nível $E_{n}$. Denotamos $\mathbf{x}_{\max }=\left(x_{\max , n}\right)_{n \geq 1}$ e $\mathbf{x}_{\min }=\left(x_{\min , n}\right)_{n \geq 1}$. Note que estes elementos estão unicamente determinados e estão em $X_{\mathfrak{B}}$.

Seja $\varphi_{\mathfrak{B}}: X_{\mathfrak{B}} \rightarrow X_{\mathfrak{B}}$ o homeomorfismo tal que

$$
\varphi_{\mathfrak{B}}\left(\mathbf{x}_{\max }\right)=\mathbf{x}_{\min }
$$


e se $\mathbf{x} \neq \mathbf{x}_{\max }$

$$
\varphi_{\mathfrak{B}}(\mathbf{x})=\left(x_{(\min , 1)}, \ldots, x_{(\min , n-1)}, x_{(\max , n)}, x_{n+1}, \ldots\right)
$$

onde $n$ é a primeira entrada de $\mathbf{x}$ que não é uma aresta máxima. A função $\varphi_{\mathfrak{B}}$ se assemelha com o odômetro diádico.

Denominamos $\varphi_{\mathfrak{B}}$ de função de Bratteli-Vershik associada a $\mathfrak{B}$. O homeomorfismo

$$
h: X_{\mathfrak{B}} \rightarrow\{0,1\}^{\mathbb{N}}, \quad h\left(\left(x_{i_{n}, n}\right)_{n \geq 1}\right)=\left(i_{n}\right)_{n \geq 1}, \quad i_{n} \in\{0,1\} \text { para todo } n \geq 1
$$

é uma conjugação entre os sistemas $\left(\{0,1\}^{\mathbb{N}}, \Phi\right)$ e $\left(X_{\mathfrak{B}}, \varphi_{\mathfrak{B}}\right)$. Isto é, $h: X_{\mathfrak{B}} \rightarrow$ $\{0,1\}^{\mathbb{N}}$ é um homeomorfismo e

$$
h \circ \varphi_{\mathfrak{B}}=\Phi \circ h
$$

Portanto, a um sistema dinâmico minimal associamos um diagrama de Bratteli.

Ainda no exemplo acima, para cada vértice $v_{n}$ consideramos o grupo abeliano

$$
\mathbb{Z} v_{n}=\left\{m v_{n} \mid m \in \mathbb{Z}\right\}
$$

Definimos, para cada $n>0$ um homomorfismo $h_{\mathfrak{B}_{n}}: \mathbb{Z} v_{n-1} \rightarrow \mathbb{Z} v_{n}$ por

$$
h_{\mathfrak{B}_{n}}\left(m v_{n-1}\right)=2 m v_{n} .
$$

Dado $\ell>0$, definimos, também

$$
h_{\mathfrak{B}_{n}}^{\ell}=h_{\mathfrak{B}_{\ell}} \circ \ldots h_{\mathfrak{B}_{n+2}} \circ h_{\mathfrak{B}_{n+1}}
$$

Repare que, pela definição do diagrama de Bratteli $\mathfrak{B}$, para todo $n>0$, existem duas arestas ligando o vértice $v_{n-1}$ ao vértice $v_{n}$. Consideramos então, a união disjunta dos grupos $\mathbb{Z} v_{n}$ :

$$
\bigsqcup_{n} \mathbb{Z} v_{n}=\left\{\left(m v_{n}, n\right) \mid n \geq 0, m \in \mathbb{Z}\right\}
$$

e estabelecemos uma relação de equivalência em $\bigsqcup_{n} \mathbb{Z} v_{n}$, onde dois elementos $\left(v_{n}, n\right)$ e $\left(v_{k}, k\right)$ são equivalentes se exitem inteiros não negativos $\ell$ e $k$ tais que

$$
h_{\mathfrak{B}_{n}}^{\ell}\left(v_{n}\right)=h_{\mathfrak{B}_{m}}^{k}\left(v_{m}\right) \quad \text { e } n+\ell=m+k .
$$

O grupo formado por todas as classes de equivalência desta relação é chamado de grupo de dimensão do diagrama de Bratteli $\mathfrak{B}$ e denotado por $D(\mathfrak{B})$. 
Mostraremos que $D(\mathfrak{B})$ é isomorfo ao grupo diádico

$$
\left\{\frac{p}{2^{n}} \mid p \in \mathbb{Z}, n \in \mathbb{N}\right\}
$$

Podemos representar a relação de equivalência $R_{\mathfrak{B}}$ pelo conjunto

$$
R_{\mathfrak{B}}=\left\{(\mathbf{x}, \mathbf{y}) \in X_{\mathfrak{B}} \times X_{\mathfrak{B}} \mid \mathbf{x} \sim_{\mathfrak{B}} \mathbf{y}\right\}
$$

Fixe $N>0$, denotamos o conjunto dos caminhos que ligam o vértice $v_{0}$ ao vértice $v_{N}$ por $E_{0, N}$ e um elemento de $E_{0, N}$ por $p=\left(p_{1}, \ldots, p_{N}\right)$. Definimos o cilindro centrado em $p$ por

$$
C(p)=\left\{\mathbf{x} \in X_{\mathfrak{B}} \mid\left(x_{1}, \ldots, x_{N}\right)=\left(p_{1}, \ldots, p_{N}\right)\right\}
$$

Agora, dados $p, q \in E_{0, N}$, considere o conjunto

$$
\gamma(p, q)=\left\{(\mathbf{x}, \mathbf{y}) \mid \mathbf{x} \in C(p), \mathbf{y} \in C(q) \text { e } x_{n}=y_{n} \text { para todo } n>N\right\}
$$

Provamos que a coleção formada pelo conjunto vazio e por todos os conjuntos $\gamma(p, q)$, onde $p, q \in E_{0, N}$ e $N \geq 1$, gera uma base para uma topologia do conjunto $R_{\mathfrak{B}}$ denominada étale. Observamos que a topologia étale não é a mesma que a induzida pela topologia produto.

Considere o grupo quociente

$$
D\left(X_{\mathfrak{B}}, R_{\mathfrak{B}}\right)=C\left(X_{\mathfrak{B}}, \mathbb{Z}\right) / B\left(X_{\mathfrak{B}}, R_{\mathfrak{B}}\right)
$$

onde $C\left(X_{\mathfrak{B}}, \mathbb{Z}\right)$ é o grupo das funções contínuas de $X_{\mathfrak{B}}$ em $\mathbb{Z}$ e $B\left(X_{\mathfrak{B}}, R_{\mathfrak{B}}\right)$ o subgrupo de $C\left(X_{\mathfrak{B}}, \mathbb{Z}\right)$ gerado pelas funções da forma $\mathcal{X}_{\pi_{2}(\gamma(p, q))}-\mathcal{X}_{\pi_{1}(\gamma(p, q))}$, onde $\mathcal{X}$ é a função característica e $\pi_{1}$ e $\pi_{2}$ são, respectivamente, as projeções na primeira e na segunda coordenada. Provamos que $D\left(X_{\mathfrak{B}}, R_{\mathfrak{B}}\right)$ é isomorfo ao grupo de dimensão $D(\mathfrak{B})$, portanto, também é isomorfo ao grupo diádico.

Agora que os conceitos já foram apresentados para um exemplo, vamos apresentá-los em um caso geral.

\subsection{2}

\section{Diagramas de Bratteli e sistemas de Cantor minimais}

Um Diagrama de Bratteli é um grafo infinito, denotado por

$$
\mathfrak{B}=\left(V=\left\{V_{n}\right\}_{n \geq 0}, E=\left\{E_{n}\right\}_{n \geq 1}, s=\left\{s_{n}\right\}_{n \geq 1}, r=\left\{r_{n}\right\}_{n \geq 1}\right),
$$


onde $V$ é conjunto dos vértices, $E$ o conjunto de pares e $V_{n}$ e $E_{n}$ são conjuntos finitos para todo $n$. Para cada $n>1$, temos que $s_{n}: E_{n} \rightarrow V_{n-1}$ e $r_{n}: E_{n} \rightarrow V_{n}$. Além disso, $s^{-1}\{v\} \neq \emptyset$ para todo $v \in \cup_{n \geq 1} V_{n}$ e $r^{-1}\{v\} \neq \emptyset$ para todo $v \in \cup_{n \geq 0} V_{n}$, isto é, não existe vértice que está desconectado. Assim, é possível formar caminhos infinitos com as arestas de $\mathfrak{B}$. Denotamos o espaço dos caminhos infinitos de $\mathfrak{B}$ por $X_{\mathfrak{B}}$.

Dizemos que duas arestas e e $w$ entre os níveis $V_{n-1}$ e $V_{n}$ são comparáveis se, e só se, incidem sobre o mesmo vértice em $V_{n}$, isto é, $r(e)=r(w)$. Assim, é possível ordenar as arestas que são comparáveis em $E_{n}$ e é possível determinar um elemento máximo e um elemento mínimo. Neste caso, $\mathfrak{B}$ é um diagrama de Bratteli ordenado.

O diagrama de Bratteli da Figura 1.2 possui apenas um caminho infinito formado somente por arestas máximas e um caminho infinito formado por arestas mínimas, mas há casos em que isso não ocorre. Por exemplo, na Figura 1.3, apresentamos um grafo em que isto não ocorre, já que todo vértice só recebe uma aresta, toda aresta é máxima e mínima ao mesmo tempo. Assim, temos mais de um caminho infinito formado somente por arestas máximas e mais de um caminho infinito formado somente por arestas mínimas.

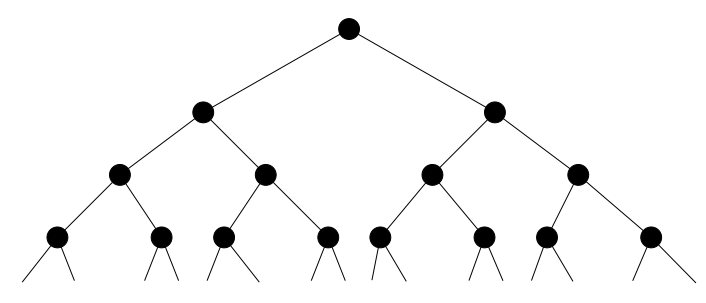

Figura 1.3: Diagrama de Bratteli que não é próprio

Se existir uma ordem em que o caminho infinito formado apenas por aresta máxima e o caminho infinito formado apenas por arestas mínimas são únicos, dizemos que $\mathfrak{B}$ é um diagrama de Bratteli ordenado e próprio. Denotamos por $\mathbf{x}_{\max }$ e por $\mathbf{x}_{\min }$ os caminhos formados somente por arestas máximas e mínimas, respectivamente.

Suponha que $\mathfrak{B}$ é um diagrama de Bratteli ordenado e próprio. Dado $\mathbf{x} \neq \mathbf{x}_{\max }$, existe $n>0$ tal que $x_{n}$ não é uma aresta máxima, considere que $n$ é mínimo com tal propriedade. Seja $y_{n}$ comparável com $x_{n}$ e seguinte a $x_{n}$ na ordem de $\mathfrak{B}$ e $\left(y_{1}, \ldots, y_{n-1}\right)$ um caminho formado somente por arestas mínimas 
ligando $v_{0}$ ao vértice que se origina a aresta $y_{n}$, definimos o homeomorfismo

$$
\begin{aligned}
\varphi_{\mathfrak{B}} & : X_{\mathfrak{B}} \rightarrow X_{\mathfrak{B}} \\
& \varphi_{\mathfrak{B}}\left(\mathbf{x}_{\max }\right)=\mathbf{x}_{\min }, \\
& \varphi_{\mathfrak{B}}(\mathbf{x})=\left(y_{1}, \ldots, y_{n}, x_{n+1}, x_{n+2}, \ldots\right), \text { se } \mathbf{x} \neq \mathbf{x}_{\max }
\end{aligned}
$$

Denominamos o homeomorfismo $\varphi_{\mathfrak{B}}$ de função de Bratteli-Vershik associada a $\mathfrak{B}$. Pela definição da função $\varphi_{\mathfrak{B}}$, temos que

$$
\varphi\left(y_{1}, \ldots, y_{n}, x_{n+1}, x_{n+2}, \ldots\right)=\left(\tilde{y}_{1}, y_{2}, y_{3}, \ldots, y_{n}, x_{n+1}, x_{n+2} \ldots\right\}
$$

onde $\tilde{y}_{1}$ é o elemento seguinte a $y_{1}$ na ordem considerada.

Mais uma vez, a função $\varphi_{\mathfrak{B}}$ é parecida com o odômetro diádico, mas desta vez há mais opções para as entradas.

Provaremos, que dados um conjunto de Cantor $X$ e um homeomorfismo minimal $\varphi: X \rightarrow X$, existe um diagrama de Bratteli ordenado e próprio $\mathfrak{B}$ tal que $(X, \varphi)$ e $\left(X_{\mathfrak{B}}, \varphi_{\mathfrak{B}}\right)$ são conjugados.

,Se $\gamma: U \rightarrow V$ é um homeomorfismo e $U, V \subset X$ são abertos, dizemos que $\gamma$ é um homeomorfismo parcial. Dizemos que $\Gamma$ é uma ação loca, se $\Gamma$ é uma coleção de homeomorfismos parciais em $X$ tal que:

1. se $\gamma \in \Gamma$, então $\gamma^{-1} \in \Gamma$;

2. se $\gamma_{1}$ e $\gamma_{2}$ estão em $\Gamma$, então $\gamma_{1} \circ \gamma_{2}$ e $\gamma_{1} \cap \gamma_{2}$ estão em $\gamma$;

3. a coleção de conjuntos

$$
\left\{U \subset X \mid U \text { é aberto e } \operatorname{id}_{U} \in \Gamma\right\}
$$

forma uma base para a topologia de $X$.

Para cada $\gamma \in \Gamma$, denotamos por $U_{\gamma}$ o seu domínio e representamos $\gamma$ como o par $\left(U_{\gamma}, \gamma\left(U_{\gamma}\right)\right)$. Veremos que a propriedade de que a interseção de dois homeomorfismo parciais de $\Gamma$ está em $\Gamma$ fornece informações topológicas interessantes.

Então definimos a relação de equivalência $R_{\Gamma}$ em $X$, onde dois elementos $x$ e $y$ estão associados, se existe $\gamma \in \Gamma$ tal que $y=\gamma(x)$. Podemos representar a relação $R_{\Gamma}$ como o subconjunto

$$
R_{\Gamma}:=\left\{(x, y) \in X \times X \mid(x, y) \in \bigcup_{\gamma \in \Gamma}\{(x, \gamma(x))\}, x \in U_{\gamma}\right\} \subset X \times X
$$


Provaremos que $\Gamma$ é base de uma topologia em $R_{\Gamma}$. Denominamos tal topologia de étale e $R_{\Gamma}$ de relação de equivalência étale. Dois exemplos de relação de equivalência étale são a relação de equivalência orbital e a relação de equivalência $R_{\mathfrak{B}}$, onde $\mathfrak{B}$ é um diagrama de Bratteli. Dizemos que duas relações de equivalência étale $R$ e $Q$ definidas em $X$ e $Y$, respectivamente, são isomorfas se existe um homeomorfismo $g: X \rightarrow Y$ tal que $g \times g: R \rightarrow Q$ é um homeomorfismo quando consideradas as topologias étales de $R$ e $Q$.

Dada uma relação de equivalência étale $R$ em um espaço métrico $X$, definimos o grupo quociente

$$
D(X, R)=C(X, \mathbb{Z}) / B(X, R)
$$

onde $C(X, \mathbb{Z})$ é grupo das funções contínuas de $X$ em $\mathbb{Z}$ e $B(X, R)$ o subgrupo de $C(X, \mathbb{Z})$ gerado pelas funções da forma $\mathcal{X}_{\pi_{2}(\gamma)}-\mathcal{X}_{\pi_{1}(\gamma)}$, onde $\pi_{1}$ e $\pi_{2}$ são, respectivamente, as projeções na primeira e na segunda coordenada e $\gamma \subset R$ é um homeomorfismo parcial compacto e aberto. Provamos que este grupo quociente é um invariante algébrico para relações de equivalência étale em espaços compactos e totalmente desconexos. Isto é, se $(X, R)$ e $(Y, Q)$ são relações de equivalência étale isomorfas, então os grupos $D(X, R)$ e $D(Y, Q)$ são isomorfos.

Dado um grupo abeliano $(G,+)$, dizemos que $G^{+}$é o cone positivo de $G$, se $G^{+}$é fechado para a operação de $G$,

$$
G^{+}-G^{+}=G \quad \text { e } \quad G^{+} \bigcap\left(-G^{+}\right)=\{0\} .
$$

Se o grupo $G$ possui um cone positivo, dizemos que $\left(G, G^{+}\right)$é um grupo abeliano ordenado. Dados $a, b \in G$, definimos que $a \leq b$ se, e só se, $b-a \in G^{+}$.

Dado um diagrama de Bratteli $\mathfrak{B}=\left(V=\left\{V_{n}\right\}_{n \geq 0}, E=\left\{E_{n}\right\}_{n \geq 1}, r, s\right)$, para cada $n \geq 0$, considerando os conjunto

$$
\mathbb{Z} V_{n}=\left\{\sum_{i=1}^{\# V_{n}} m_{i} v_{i}^{n} \mid m_{i} \in \mathbb{Z} \text { para todo } 1 \leq i \leq \# V_{n}\right\}
$$

e

$$
\mathbb{Z} V_{n}^{+}=\left\{\sum_{i=1}^{\# V_{n}} m_{i} v_{i}^{n} \mid m_{i} \geq 0 \text { para todo } 1 \leq i \leq \# V_{n}\right\}
$$

o par $\left(\mathbb{Z} V_{n}, \mathbb{Z} V_{n}\right)^{+}$forma um grupo abeliano ordenado.

Consideramos a união disjunta dos grupos $\mathbb{Z} V_{n}$

$$
\bigsqcup_{n} \mathbb{Z} V_{n}=\left\{(a, n) \mid n \geq 0, a \in \mathbb{Z} V_{n}\right\}
$$


e para cada $n>0$ um homomorfismo $h_{\mathfrak{B}_{n}}: \mathbb{Z} V_{n-1} \rightarrow \mathbb{Z} V_{n}$, definido por

$$
h_{\mathfrak{B}_{n}}\left(\sum_{i=1}^{\# V_{n-1}} m_{i} v_{i}^{n-1}\right)=\sum_{j=1}^{\# V_{n}}\left(\sum_{i=1}^{\# V_{n-1}} \#\left(s^{-1}\left\{v_{i}^{n-1}\right\} \cap r^{-1}\left\{v_{j}^{n}\right\}\right) m_{i}\right) v_{j}^{n} .
$$

Note que, dados $1 \leq i \leq \# V_{n-1}$ e $1 \leq j \leq \# V_{n+1}$ o número

$$
\#\left(s^{-1}\left\{v_{i}^{n-1}\right\} \cap r^{-1}\left\{v_{j}^{n}\right\}\right)
$$

é a quantidade de arestas que ligam o vértice $v_{i}^{n-1}$ ao vértice $v_{j}^{n}$. No caso do diagrama de Bratteli da Figura 1.2, há sempre duas arestas ligando vértices de níveis consecutivos.

Dado $\ell>0$, definimos a composição $h_{\mathfrak{B}, n}^{\ell}$ como no caso anterior.

Estabelecemos, também, uma relação de equivalência em $\bigsqcup_{n} \mathbb{Z} V_{n}$, onde dois elementos $(a, n)$ e $(b, m)$ estão relacionados se, e só se, existem inteiros não negativos $k$ e $\ell$, tais que

$$
h_{\mathfrak{B}_{n}}^{k}(a)=h_{\mathfrak{B}_{m}}^{\ell}(b) \quad \text { e } n+k=m+\ell .
$$

Definimos o conjunto de todas as classes de equivalência desta relação como o grupo de dimensão do diagrama de Bratteli $\mathfrak{B}$, denotado por $D(\mathfrak{B})$.

Provamos que se $\mathfrak{B}$ é um diagrama de Bratteli tal que $V_{0}$ possui somente um elemento, os invariantes $D\left(X_{\mathfrak{B}}, R_{\mathfrak{B}}\right)$ e $D\left(X_{\mathfrak{B}}, R_{\varphi_{\mathfrak{B}}}\right)$ são isomorfos ao grupo de dimensão $D(\mathfrak{B})$.

Um grupo abeliano ordenado $\left(G, G^{+}\right)$é dito perfurado se existem um $a \in G$ e um inteiro positivo $n$ tais que $a \notin G^{+}$e $n a \in G^{+}$. Caso contrário, dizemos que $\left(G, G^{+}\right)$é sem perfuração.

Agora, dizemos que $\left(G, G^{+}\right)$satisfaz a interpolação de Riesz se dados quaisquer $a, b, c, d \in G$, tais que $a, b \leq c, d$, existe $e \in G$ tal que $a, b \leq e \leq c, d$.

Um dos principais teoremas apresentados é o Teorema de EffrosHandelman-Shen que determina sob quais condições um grupo abeliano ordenado é o grupo de dimensão de algum diagrama de Bratteli. Este teorema é útil, porque através dele podemos obter diagramas de Bratteli, simplesmente, verificando se um grupo abeliano é enumerável, sem perfuração e satisfaz a interpolação de Riesz.

Outro teorema fundamental é o Teorema de Bratteli-Elliot-Krieger. Este teorema informa quando dados dois diagramas de Bratteli $\mathfrak{B}$ e $\mathfrak{T}$ os invariantes $D\left(X_{\mathfrak{B}}, R_{\mathfrak{B}}\right)$ e $D\left(X_{\mathfrak{T}}, R_{\mathfrak{T}}\right)$ são invariantes completos, isto é, as relações de equivalência $R_{\mathfrak{B}}$ e $R_{\mathfrak{T}}$ são isomorfas se, e só se, os invariantes $D\left(X_{\mathfrak{B}}, R_{\mathfrak{B}}\right)$ e $D\left(X_{\mathfrak{T}}, R_{\mathfrak{T}}\right)$ são isomorfos. 
Agora, voltamos às relações de equivalência. Dizemos que duas relações de equivalência $R$ e $Q$ definidas nos conjuntos de Cantor $X$ e $Y$, respectivamente, são equivalentes se existe um homeomorfismo $h: X \rightarrow Y$ tal que $h\left(\langle x\rangle_{R}\right)=$ $\langle h(x)\rangle_{Q}$. Em particular, se $\varphi: X \rightarrow X$ e $\psi: Y \rightarrow Y$ são homeomorfismo minimais e as relações orbitais $R_{\varphi}$ e $R_{\psi}$ são equivalentes dizemos que o homeomorfismo $h: X \rightarrow Y$ é uma equivalência orbital entre $(X, \varphi)$ e $(Y, \psi)$. Provamos que $h: X \rightarrow Y$ é uma equivalência orbital entre $(X, \varphi)$ e $(Y, \psi)$ se e somente se existem funções, denominadas cociclos orbitais, $m_{h}: X \rightarrow \mathbb{Z}$ e $n_{h}: Y \rightarrow \mathbb{Z}$ tais que

$$
h \circ \varphi(x)=\psi^{m_{h}(x)} \circ h(x), \quad \text { para todo } x \in X
$$

e

$$
h^{-1} \circ \psi(y)=\varphi^{n_{h}(y)} \circ h^{-1}(y), \quad \text { para todo } y \in Y .
$$

Uma equivalência orbital tal que seus cociclos orbitais possuem, cada um, no máximo um ponto de descontinuidade é dita forte.

Provamos, usando o Teorema de Bratteli-Elliot-Krieger, que dados conjuntos de Cantor $X$ e $Y$ e homeomorfismos minimais $\varphi: X \rightarrow X$ e $\psi: Y \rightarrow Y$, os invariantes $D\left(X, R_{\varphi}\right)$ e $D\left(Y, R_{\psi}\right)$ são isomorfos se e somente se existe uma equivalência orbital forte entre $(X, \varphi)$ e $(Y, \psi)$.

Concluímos a dissertação com uma breve discussão sobre quando uma equivalência orbital entre $(X, \varphi)$ e $(Y, \psi)$ é uma conjugação entre os sistemas $(X, \varphi)$ e $(Y, \psi)$.

\section{2}

\section{Organização desta dissertação}

Abaixo segue uma descrição específica do que é feito em cada capítulo:

No Capítulo 2, definiremos a função odômetro, a relação orbital associada ao odômetro e a relação de equivalência de caudas. Provaremos que a relação orbital associada ao odômetro é minimal. Além disto, estudaremos como a relação orbital e a relação de equivalência de caudas se relacionam.

No Capítulo 3, definiremos um conjunto de Cantor e conceitos necessário para mostrar que quaisquer dois conjuntos de Cantor são homeomorfos.

No Capítulo 4, definiremos a relação de equivalência orbital e o conceito de equivalência orbital entre duas relações de equivalência. Dados $X$ e $Y$ espaços conexos e homeomorfismos $\varphi: X \rightarrow X$ e $\psi: Y \rightarrow Y$ sem pontos periódicos, mostraremos que se existe uma equivalência orbital entre $R_{\varphi}$ e $R_{\psi}$, então existe uma conjugação entre $(X, \varphi)$ e $(Y, \psi)$ ou entre $(X, \varphi)$ e $\left(Y, \psi^{-1}\right)$. 
No Capítulo 5, definiremos na Seção 5.1 um diagrama de Bratteli e mostraremos que o espaço dos caminhos infinitos formado pelas arestas de um digrama de Bratteli, munido com uma métrica apropriada, é compacto. Dado um diagrama de Bratteli $\mathfrak{B}$ explicaremos como obter um novo diagrama de Bratteli a partir de $\mathfrak{B}$ através de uma subsequência. Estabeleceremos um homeomorfismo entre $X_{\mathfrak{B}}$ e o espaço dos caminhos infinitos formado pelas arestas deste novo diagrama. Definiremos a relação de equivalência AF e o que é um diagrama de Bratteli simples.

No Capítulo 6, definiremos, em primeiro lugar, um diagrama de Bratteli ordenado e próprio e a aplicação de Bratteli-Vershik. Também estudaremos o comportamento da relação orbital $R_{\varphi_{\mathfrak{B}}}$ quando $\mathfrak{B}$ é um diagrama de Bratteli ordenado e próprio.

No Capítulo 7, dados um conjunto de Cantor $X$ e um homeomorfismo minimal $\varphi: X \rightarrow X$, construiremos um diagrama de Bratteli $\mathfrak{B}$ ordenado simples e próprio, tal que os sistemas dinâmicos $(X, \varphi)$ e $\left(X_{\mathfrak{B}}, \varphi_{\mathfrak{B}}\right)$ são topologicamente conjugados. Para isto, definiremos a partição de KakutaniRokhlin, ou castelo, de um conjunto de Cantor $X$ e uma sequência de castelos refinadores de $X$. Mostraremos, também, a existência de uma sequência de castelos refinadores de um conjunto de Cantor. Construiremos o diagrama de Bratteli $\mathfrak{B}$ e definimos a conjugação entre $(X, R)$ e $\left(X_{\mathfrak{B}}, R_{\mathfrak{B}}\right)$.

No Capítulo 8, definiremos uma ação local, uma relação de equivalência étale e quando dois espaços $\left(X, R_{\Gamma}, \Gamma\right)$ e $\left(Y, Q_{\Upsilon}, \Upsilon\right)$ são isomorfos, onde $X$ e $Y$ são espaços métricos, $\Gamma$ e $\Upsilon$ ações locais e $R_{\Gamma}$ e $Y_{\Upsilon}$ são as relações de equivalência étale induzidas por $\Gamma$ e $\Upsilon$. Mostraremos que $R_{\mathfrak{B}}$ é uma relação de equivalência étale. Também definiremos o que é uma relação de equivalência AF em um espaço topológico qualquer $X$. Provaremos sob quais condições uma relação de equivalência étale definida em um conjunto de Cantor é uma relação de equivalência AF. Mostraremos que a relação orbital é uma relação de equivalência étale e que dados um conjunto de Cantor e um homeomorfismo minimal $\varphi: X \rightarrow X$, não existe nenhum diagrama de Bratteli $\mathfrak{B}$ tal que as relações $R_{\varphi}$ e $R_{\mathfrak{B}}$ são isomorfas.

Os objetos algébricos necessários para o que será feito a seguir serão tratados no Capítulo 9.

No Capítulo 10, definiremos o invariante algébrico $D(X, R)$, onde $X$ é um espaço métrico compacto e totalmente desconexo e $R$ é uma relação de equivalência étale definida em $X$. Provaremos que se $R$ e $Q$ são relações de equivalência étale definidas em $X$ e $Y$, respectivamente, tais que $(X, R)$ e $(Y, Q)$ são isomorfos então existe um isomorfismo ordenado entre os invariantes $D(Y, Q)$ e $D(X, R)$. 
No Capítulo 11, dado um diagrama de Bratteli $\mathfrak{B}$ definiremos o grupo de dimensão $D(\mathfrak{B})$ do diagrama de Bratteli $\mathfrak{B}$. Mostraremos que dado um diagrama de Bratteli $\mathfrak{B}$ e um diagrama de Bratteli $\mathfrak{T}$ obtido através de $\mathfrak{B}$ a partir de uma subsequência existe um isomorfismo entre $D(\mathfrak{B})$ e $D(\mathfrak{T})$.

No Capítulo 12 , estudaremos o invariante $D\left(X_{\mathfrak{B}}, R\right)$ quando $R$ é a relação $R_{\mathfrak{B}}$ e quando $R$ é a relação $R_{\varphi_{\mathfrak{B}}}$.

Nos Capítulos 13 e 14, provaremos o Teorema de Effros-Handelman-Shen e o Teorema de Bratteli-Elliot-Krieger, respectivamente.

No Capítulo 15, na Seção 15.1 definiremos o que são os cociclos orbitais associados a uma equivalência orbital. Na seção 15.2 definiremos o que é uma equivalência orbital forte e provaremos que dados conjuntos de Cantor $X$ e $Y$ e homeomorfismos minimais $\varphi: X \rightarrow X$ e $\psi: Y \rightarrow Y$, existe uma equivalência orbital forte entre $(X, \varphi)$ e $(Y, \psi)$ se e somente se existe um isomorfismo ordenado que preserva ordem unitária entre os invariantes $D\left(X, R_{\varphi}\right)$ e $D(Y, \psi)$.

Finalmente, no capítulo 16, apresentaremos um exemplo de quando uma equivalência orbital não é uma conjugação. 


\section{Odômetro}

O objetivo deste capítulo é definir a função odômetro e provar a sua minimalidade.

\section{1}

\section{Adição de sequências}

Denotamos por $\{0,1\}^{\mathbb{N}}$ o espaço das sequências infinitas de $0^{\prime} s$ e $1^{\prime} s$ e um elemento de $\{0,1\}^{\mathbb{N}}$ por $\mathbf{x}=\left(x_{n}\right)_{n \geq 1}$, tal que $x_{n} \in\{0,1\}$ para todo $n \geq 1$. Com o intuito de definir a função odômetro, precisamos definir a soma de dois elementos neste espaço, o que não pode ser feito da mesma forma que somamos números representados na base decimal, já que as sequências são infinitas e, portanto, não conseguimos determinar quem é o último elemento a direita. Por isso, precisamos somar da esquerda para direita. Dados dois elementos $\mathbf{x}=\left(x_{n}\right)_{n \geq 1}$ e $\mathbf{y}=\left(y_{n}\right)_{n \geq 1}$ em $\{0,1\}^{\mathbb{N}}$, definimos que

$$
\mathbf{x}+\mathbf{y}=\mathbf{z}=\left(z_{n}\right)_{n \geq 1}
$$

e consideramos a sequência auxiliar $\left\{w_{n}\right\}_{n \geq 1}$, onde se $x_{1}+y_{1}<2$, então $z_{1}=x_{1}+y_{1}$ e definimos $w_{1}=0$. Agora, se $x_{1}+y_{1}=2$, então $z_{1}=0$ e definimos $w_{1}=1$. Considere, agora, que $n>1$ e que já foram definidos os valores de $z_{n-1}$ e $w_{n-1}$. Se $x_{n}+y_{n}+w_{n-1}<2$, então $z_{n}=x_{n}+y_{n}+w_{n-1}$ e definimos que $w_{n}=0$. Caso, $x_{n}+y_{n}+w_{n-1} \geq 2$, temos que $z_{n}=x_{n}+y_{n}+w_{n-1}-2$ e definimos $w_{n}=1$. Dessa forma, quando somamos duas entradas iguais a 10 resultado é 0 e transportamos 1 para a soma das entradas seguintes.

\section{2}

\section{Odômetro}

Dado um espaço métrico $(X, d)$ e um homeomorfismo $\varphi: X \rightarrow X$, definimos a órbita de um ponto $x \in X$ por $f$ como o conjunto

$$
\mathcal{O}_{\varphi}(x)=\left\{\varphi^{n}(x) \mid n \in \mathbb{Z}\right\}
$$

Dizemos que a função $\varphi$ é minimal se $\mathcal{O}_{\varphi}(x)$ é um conjunto denso para todo $x \in X$. Neste caso, dizemos que $(X, \varphi)$ é um sistema dinâmico minimal. 
A partir de agora, considere que $X=\{0,1\}^{\mathbb{N}}$ e a função $\Phi: X \rightarrow X$ definida por

$$
\Phi(\mathbf{1})=\mathbf{0} \quad \text { e } \Phi(\mathbf{x})=\mathbf{x}+(1000 \ldots), \quad \text { se } \mathbf{x} \neq \mathbf{1}
$$

O objetivo desta seção é mostrar que $(X, \Phi)$ é um sistema dinâmico minimal. Para isto, precisamos de alguns conceitos.

Considere a função $d: X \times X \rightarrow \mathbb{R}$, definida por

$$
d(\mathbf{x}, \mathbf{y})=\left\{\begin{array}{l}
1, \quad \text { se } x_{1} \neq y_{1}, \\
\inf \left\{2^{-n} \mid n \geq 1, x_{i}=y_{i}, \text { para todo } 1 \leq i \leq n\right\}, \quad \text { se } x_{1}=y_{1}
\end{array}\right.
$$

Proposição 2.2.1. A função d é uma métrica no espaço $X$.

Prova. Mostraremos que a função $d$ satisfaz as propriedades da definição de métrica. Segue direto da definição que a função $d$ satisfaz a propriedade simétrica.

Mostraremos agora que $d$ satisfaz a propriedade reflexiva. Seja $\mathbf{x} \in X$. Pela definição da função $d$, temos que

$$
d(\mathbf{x}, \mathbf{x})=\lim _{n \rightarrow \infty} 2^{-n}=0
$$

Por outro lado, considere que $\mathbf{y}$ seja um elemento de $X$ tal que $d(\mathbf{x}, \mathbf{y})=0$. Então

$$
\inf \left\{2^{-n} \mid n \geq 0, x_{i}=y_{i} \text {, para todo } 0 \leq i \leq n\right\}=0 .
$$

Logo, $x_{i}=y_{i}$ para todo $i \geq 1$. Portanto, $\mathbf{x}=\mathbf{y}$ e $d$ satisfaz a propriedade reflexiva.

Por último, provaremos que $d$ satisfaz a desigualdade triangular. Sejam $\mathbf{x}, \mathbf{y}, \mathbf{z}$ elementos de $X$. Consideraremos, primeiro, o caso em que $d(\mathbf{x}, \mathbf{y})=1$. Neste caso, $x_{1} \neq y_{1}$, então $z_{1}$ é igual ou a $x_{1}$ ou a $y_{1}$. Portanto, $d(\mathbf{x}, \mathbf{z})=1$ ou $d(\mathbf{z}, \mathbf{y})=1$. Logo

$$
d(\mathbf{x}, \mathbf{y}) \leq d(\mathbf{x}, \mathbf{z})+d(\mathbf{z}, \mathbf{y})
$$

Agora, consideraremos o caso em que $d(\mathbf{x}, \mathbf{y}) \neq 1$. Neste caso, existe $n$ tal que $x_{i}=y_{i}$ para todo $1 \leq i \leq n$. Se $z_{1} \neq x_{1}=y_{1}$, temos que $d(\mathbf{x}, \mathbf{z})=d(\mathbf{y}, \mathbf{z})=1$ e, portanto a desigualdade triangular é satisfeita. Se $z_{1}=x_{1}=y_{1}$, existe $k$ tal que $z_{i}=x_{i}$ para todo $1 \leq i \leq k$. Caso $k \leq n$, temos que

$$
d(\mathbf{x}, \mathbf{z})+d(\mathbf{y}, \mathbf{z})=\frac{2}{2^{k}} \geq \frac{1}{2^{n}}=d(\mathbf{y}, \mathbf{z})
$$


Agora, se $k>n$, temos que $y_{n+1} \neq z_{n+1}$. Então,

$$
d(\mathbf{x}, \mathbf{z})+d(\mathbf{y}, \mathbf{z})=\frac{1}{2^{k}}+\frac{1}{2^{n}} \geq \frac{1}{2^{n}}=d(\mathbf{x}, \mathbf{y}) .
$$

Concluímos, assim, que $d$ é uma métrica e, portanto $(X, d)$ é um espaço métrico.

Definição 2.2.2 (Cilindros). Seja o conjunto

$$
[\mathbf{x}]_{n}=\left\{\mathbf{y} \in X \mid y_{1}=x_{1}, y_{2}=x_{2} \ldots y_{n}=x_{n}\right\}
$$

tais conjuntos são chamados de cilindros.

Quando for conveniente, usaremos as notações:

$$
\left[a^{n-1} b\right]_{n}=\left\{\mathbf{x} \in X \mid x_{i}=\text { a para todo } 1 \leq i \leq n-1 \text { e } x_{n}=b\right\}
$$

$e$

$$
\left[x_{1} x_{2} \ldots x_{n}\right]=\left\{\mathbf{y} \in X \mid y_{1}=x_{1}, y_{2}=x_{2} \ldots y_{n}=x_{n}\right\}
$$

Os cilindros geram a base da topologia em $(X, d)$, e temos que $X$ é a união de todos os $2^{n}$ cilindros de tamanho $n$. Note que se dois cilindros de mesmo tamanho são diferentes, então eles são disjuntos.

Denominamos a função $\Phi: X \rightarrow X$, definida na equação (2.2.1) por odômetro diádico. Repare que, na prática, de acordo com a definição de soma no espaço $X$, temos,

$$
\Phi(\mathbf{x})=\left\{\begin{array}{l}
1 x_{2} x_{3} \ldots, \quad \text { se } x_{1}=0 \\
00 \ldots\left(x_{i}=1\right) x_{i+1} x_{i+2} \ldots, \quad \text { se } x_{k}=1 \text { para todo } k<i \text { e } x_{i}=0 \\
\Phi(\mathbf{1})=\mathbf{0} .
\end{array}\right.
$$

Lema 2.2.3. Sejam $\mathbf{x}$ e $\mathbf{x}^{\prime} \in X$, definimos $\mathbf{y}=\Phi(\mathbf{x})$ e $\mathbf{y}^{\prime}=\Phi\left(\mathbf{x}^{\prime}\right)$. Se $x_{n}=x_{n}^{\prime}$ para todo $1 \leq n \leq N$ então $y_{n}=y_{n}^{\prime}$ para todo $1 \leq n \leq N$.

Prova. Considere que $i$ e $j$ são as primeiras entradas tais que $x_{i}=0$ e $x_{j}^{\prime}=0$. Consideraremos dois casos. O primeiro é quando $i=j$. Neste caso, $1 \leq i, j \leq N$. Pela definição da função $\Phi$,

$$
(\Phi(\mathbf{x}))_{\ell}=\left(\Phi\left(\mathbf{x}^{\prime}\right)\right)_{\ell}=0 \text { para todo } \ell<i, j, \quad(\Phi(\mathbf{x}))_{i}=\left(\Phi\left(\mathbf{x}^{\prime}\right)\right)_{j}=1
$$

e as demais entradas permanecem inalteradas. Portanto, $y_{n}=y_{n}^{\prime}$ para todo $1 \leq n \leq N$. 
Agora, consideramos o caso em que $i \neq j$. Neste caso temos que $N<i, j$, assim $y_{n}=y_{n}^{\prime}$ para todo $1 \leq n \leq N$. Concluímos, assim, a demonstração do lema.

Proposição 2.2.4. A função $\Phi$ é um homeomorfismo.

Prova. Considere a função $\varphi: X \rightarrow X$, definida por:

$$
\varphi(\mathbf{x})=\left\{\begin{array}{l}
0 x_{2} x_{3} \ldots, \quad \text { se } x_{1}=1 \\
11 \ldots\left(x_{i}=0\right) x_{i+1} x_{i+2} \ldots, \quad \text { se } x_{k}=0 \text { para todo } k<i \text { e } x_{i}=1 \\
\mathbf{1} \quad \text { se } \mathbf{x}=\mathbf{0}
\end{array}\right.
$$

Lema 2.2.5. A função $\varphi$ é a função inversa de $\Phi$.

Prova. Mostraremos que para todo $\mathrm{x} \in X$,

$$
\varphi \circ \Phi(\mathbf{x})=\mathbf{x} \quad \text { e } \quad \Phi \circ \varphi(\mathbf{x})=\mathbf{x}
$$

Seja $\mathbf{x} \in X$. Temos que

$\varphi(\Phi(\mathbf{x}))=\left\{\begin{array}{l}\varphi\left(1 x_{2} x_{3} \ldots\right), \quad \text { se } x_{1}=0, \\ \varphi\left(00 \ldots\left(x_{i}=1\right) x_{i+1} x_{i+2} \ldots\right), \quad \text { se } x_{k}=1 \text { para todo } k<i \text { e } x_{i}=0, \\ \varphi(\mathbf{0}) \quad \text { se } \mathbf{x}=\mathbf{1} .\end{array}\right.$

Pela definição da função $\varphi$, segue que

$\varphi(\Phi(\mathbf{x}))=\left\{\begin{array}{l}0 x_{2} x_{3} \ldots, \quad \text { se } x_{1}=0, \\ 11 \ldots\left(x_{i}=0\right) x_{i+1} x_{i+2} \ldots, \\ \mathbf{1} \quad \text { se } \mathbf{x}=\mathbf{1} .\end{array}\right.$

Então, $\varphi(\Phi(\mathbf{x}))=\mathbf{x}$. Analogamente, $\Phi(\varphi(\mathbf{x}))=\mathbf{x}$. Concluímos que $\varphi$ é a inversa da função $\Phi$.

Pelo Lema 2.2.5, $\Phi$ possui inversa e portanto é bijetiva. Com isso, resta provar que $\Phi$ e $\Phi^{-1}=\varphi$ são contínuas.

Dados $\mathbf{x}$ e $\mathbf{y} \in X$ tais que $[\mathbf{x}]_{N}=[\mathbf{y}]_{N}$, pelo Lema 2.2.3, temos que $[\Phi(\mathbf{x})]_{N}=[\Phi(\mathbf{y})]_{N}$. Então, dado $\epsilon>0$, tomemos $\delta=\epsilon$. Logo, se $d\left(\mathbf{x}, \mathbf{x}^{\prime}\right)<\delta$ então $d\left(\Phi(\mathbf{x}), \Phi\left(\mathbf{x}^{\prime}\right)\right)<\epsilon$. Com isso $\Phi$ é contínua. Um argumento análogo mostra que $\Phi^{-1}$ também é contínua. Concluímos, então, que a função $\Phi$ é um homeomorfismo. 
Definição 2.2.6 (Relação orbital). Seja $Y$ um espaço métrico e $\varphi: Y \rightarrow Y$ um homeomorfismo. Chamaremos de $R_{\varphi}$ a relação de equivalência em $Y$ dada por:

$$
x \sim_{R_{\varphi}} y \text { se e só se existe } n \in \mathbb{Z} \text { tal que } y=\varphi^{n}(x),
$$

denominada relação orbital.

Proposição 2.2.7. A relação orbital definida acima é uma relação de equivalência.

Prova. Mostraremos que $R_{\varphi}$ satisfaz as propriedades de relação de equivalência.

- Reflexiva: $x \sim_{R_{\varphi}} x$, basta tomar $n=0$.

- Simétrica: Sejam $x, y \in X$. Se $x \sim_{R_{\varphi}} y$ então existe $n \in \mathbb{N}$ tal que $\varphi^{n}(y)=x$, como $\varphi$ é um homeomorfismo, $\varphi^{(-n)}(x)=y$ então $y \sim_{R_{\varphi}} x$.

- Transitiva: Sejam $x, y, z \in X$ tais que $x \sim_{R_{\varphi}} y$ e $y \sim_{R_{\varphi}} z$ então existem $n, m \in \mathbb{N}$ tais que $\varphi^{n}(y)=x$ e $\varphi^{m}(z)=y$. Portanto, $\varphi^{n+m}(z)=x$, com isso, $x \sim_{R_{\varphi}} z$.

Concluímos, então, que $R_{\varphi}$ é uma relação de equivalência.

Para cada $x \in Y$, denotaremos sua classe de equivalência por $\langle x\rangle_{R_{\varphi}}$. Por definição da relação $R_{\varphi}$, temos que para cada $x \in Y,\langle x\rangle_{R_{\varphi}}$ é o conjunto $\mathcal{O}_{\varphi}(x)$. Note que a relação $R_{\varphi}$ é minimal se e somente se a função $\varphi$ é desa.

Definição 2.2.8 (Minimalidade). Uma relação de equivalência é dita minimal se toda classe de equivalência é densa.

Teorema 2.2.9. Seja $\Phi$ a função odômetro então a relação $R_{\Phi}$ é minimal.

Prova. Precisaremos de alguns resultados preliminares.

Lema 2.2.10. Para todo $k \geq 0$ temos que $\Phi^{2^{k}}\left([\mathbf{0}]_{k+1}\right)=\left[0^{k} 1\right]_{k+1}$ e que $\Phi^{2^{k}}\left(\left[0^{k} 1\right]_{k+1}\right)=[\mathbf{0}]_{k+1}$.

Prova. Provaremos, primeiro, que para todo $k \geq 0$,

$$
\Phi^{2^{k}}\left([\mathbf{0}]_{k+1}\right)=\left[0^{k} 1\right]_{k+1}
$$

A demonstração será dada por indução em $k$. Segue diretamente da definição da função $\Phi$ que $\Phi\left([\mathbf{0}]_{1}\right)=[\mathbf{1}]_{1}$. Agora, suponha, por indução, que o lema vale para todo $0 \leq i \leq k$, isto é, para todo $0 \leq i \leq k$.

$$
\Phi^{2^{i}}\left([\mathbf{0}]_{i+1}\right)=\left[0^{i} 1\right]_{i+1} .
$$


Provaremos que a afirmação é verdadeira para $k+1$.

$$
\begin{aligned}
\Phi^{2^{k+1}}\left([\mathbf{0}]_{k+2}\right) & =\Phi^{1+\sum_{j=0}^{k} 2^{j}}\left([\mathbf{0}]_{k+2}\right)= \\
& =\Phi \circ \Phi \circ \Phi^{2} \circ \cdots \circ \Phi^{2^{k-1}} \circ \Phi^{2^{k}}\left([\mathbf{0}]_{k+2}\right)= \\
& =\Phi \circ \Phi \circ \Phi^{2} \circ \cdots \circ \Phi^{2^{k-1}}\left(\left[0^{k} 10\right]_{k+2}\right)=\Phi\left(\left[1^{k+1} 0\right]_{k+2}=\left[0^{k+1} 1\right]_{k+2} .\right.
\end{aligned}
$$

Acima usamos a hipótese de indução para cada $\Phi^{2^{j}}$, onde $0 \leq j \leq k$.

Mostraremos agora que $\Phi^{2^{k}}\left(\left[0^{k} 1\right]_{k+1}\right)=[\mathbf{0}]_{k+1}$ para todo $k \geq 0$.

$$
\begin{aligned}
\Phi^{2^{k+1}}\left(\left[0^{k+1} 1\right]_{k+2}\right) & =\Phi^{1+\sum_{j=0}^{k} 2^{j}}\left(\left[0^{k+1} 1\right]_{k+2}\right)= \\
& =\Phi \circ \Phi \circ \Phi^{2} \circ \cdots \circ \Phi^{2^{k-1}} \circ \Phi^{2^{k}}\left(\left[0^{k+1} 1\right]_{k+2}\right)= \\
& =\Phi \circ \Phi \circ \Phi^{2} \circ \cdots \circ \Phi^{2^{k-1}}\left(\left[0^{k} 11\right]_{k+2}\right)=\Phi\left(\left[1^{k+1} 1\right]_{k+2}\right)=[\mathbf{0}]_{k+2} .
\end{aligned}
$$

Acima usamos que $\Phi^{2^{k}}\left([\mathbf{0}]_{k+1}\right)=\left[0^{k} 1\right]_{k+1}$, para todo $k>0$. Concluímos, então, a demonstração do lema.

Corolário 2.2.11. Dado $[\mathbf{0}]_{k}$, temos que $\Phi^{2^{k}}\left([\mathbf{0}]_{k}\right)=[\mathbf{0}]_{k}$.

Prova. Note que $[\mathbf{0}]_{k}=[\mathbf{0}]_{k+1} \cup\left[0^{k} 1\right]_{k+1}$, assim:

$$
\begin{aligned}
\Phi^{2^{k}}[\mathbf{0}]_{k} & =\Phi^{2^{k}}\left([\mathbf{0}]_{k+1} \cup\left[0^{k} 1\right]_{k+1}\right)=\Phi^{2^{k}}\left([\mathbf{0}]_{k+1}\right) \cup \Phi^{2^{k}}\left(\left[0^{k} 1\right]_{k+1}\right)= \\
& =\left[0^{k} 1\right]_{k+1} \cup[\mathbf{0}]_{k+1}=[\mathbf{0}]_{k} .
\end{aligned}
$$

Finalizamos, assim, a demonstração do corolário.

Lema 2.2.12. Seja $\mathbf{x} \in X$, os cilindros $\Phi^{i}\left([\mathbf{x}]_{k}\right)$, onde $0 \leq i \leq 2^{k}-1$, são dois a dois disjuntos.

Prova. Sem perda de generalidade, mostraremos a afirmação para o cilindro $[\mathbf{0}]_{k}$. A demonstração será por indução em $k$ :

- Para $k=1$, os cilindros $[\mathbf{0}]_{1}$ e $[\mathbf{1}]_{1}$ são disjuntos, $\Phi\left([\mathbf{0}]_{1}\right)=[\mathbf{1}]_{1}$ e $\Phi\left([\mathbf{1}]_{1}\right)=[\mathbf{0}]_{1}$.

- Suponha que o lema é verdadeiro para $k$, isto é, que os cilindros $\Phi^{i}\left([\mathbf{0}]_{k}\right)$ são dois a dois disjuntos, onde $0 \leq i \leq 2^{k}-1$.

- Mostraremos que a afirmação é verdadeira pra $k+1$ :

Temos que $[\mathbf{0}]_{k+1} \subset[\mathbf{0}]_{k}$, então $\Phi^{i}\left([\mathbf{0}]_{k+1}\right) \subset \Phi^{i}\left([\mathbf{0}]_{k}\right)$, usando a hipótese de indução e que $\Phi^{i}\left([\mathbf{0}]_{k+1}\right) \subset \Phi^{i}\left([\mathbf{0}]_{k}\right)$, temos que os cilindros $\Phi^{i}\left([\mathbf{0}]_{k+1}\right)$ 
são dois a dois disjuntos para todo $0 \leq i \leq 2^{k}-1$. Pelo Lema 2.2.10, temos que que:

$$
\Phi^{2^{i}}\left([\mathbf{0}]_{k+1}\right)=\left[0^{k} 1\right]_{k+1} \subset[\mathbf{0}]_{k},
$$

então os cilindros $\Phi^{j}\left(\left[0^{k} 1\right]_{k+1}\right)$ são dois a dois disjuntos para todo $0 \leq j \leq 2^{k}-1$, mas:

$$
\Phi^{j}\left(\left[0^{k} 1\right]_{k+1}\right)=\Phi^{j}\left(\Phi^{2^{k}}\left([\mathbf{0}]_{k+1}\right)\right)=\Phi^{2^{k}+j}\left([\mathbf{0}]_{k+1}\right)
$$

assim os cilindros $\Phi^{i}\left([0]_{k+1}\right)$ são dois a dois disjuntos para todo $0 \leq i \leq$ $2^{k+1}-1$.

Observação 2.2.13. Temos que $\Phi^{i}\left([\mathbf{0}]_{k}\right)$, onde $0 \leq i \leq 2^{k}-1$ não altera as entradas $0_{\ell}$ para todo $\ell>k$, já que precisamos de $2^{k}-1$ para alterar a entrada 0 .

Continuaremos com a demonstração do Teorema 2.2.9:

Prova. Seja $\mathbf{x} \in X$, precisamos mostrar que $\langle\mathbf{x}\rangle_{\varphi}$ é um conjunto denso, isto é, temos que mostrar que dado $U \subset X$ aberto, $U \cap\langle\mathbf{x}\rangle \neq \emptyset$. Como os cilindros são uma base da topologia de $X$, dado um aberto $U$ em $X$, existe cilindro $[\mathbf{p}]_{k} \subset U$. Pelo Lema 2.2.12, todos os cilindros $\Phi^{i}\left([\mathbf{p}]_{k}\right)$ são disjuntos para todo $0 \leq i \leq 2^{k}-1$. Assim, basta mostrar que $\Phi^{2^{k}}\left([\mathbf{p}]_{k}\right)=[\mathbf{p}]_{k}$. Desta forma, a função odômetro permuta todos os cilindros de tamanho $k$ com período $2^{k}$. Portanto, a órbita de todo cilindro de tamanho $k$ contém qualquer outro cilindro de tamanho $k$ e portanto, a órbita de qualquer ponto por $\Phi$ é densa.

É suficiente mostrar a afirmação para o cilindro $[\mathbf{0}]_{k}$ isto é, mostrar que $\Phi^{2^{k}}\left([\mathbf{0}]_{k}\right)=[\mathbf{0}]_{k}$, o que já foi feito no Corolário 2.2.11. Concluímos, assim, a demonstração do teorema.

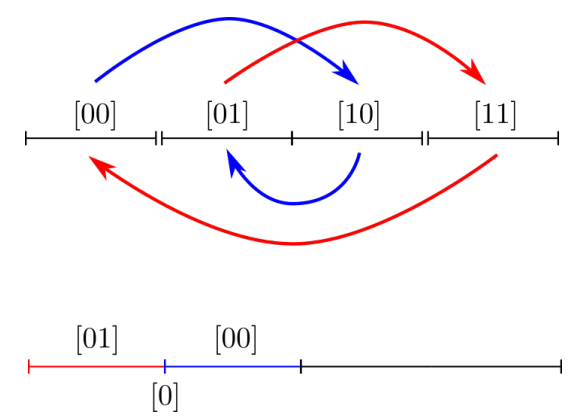

Figura 2.1: Ilustração da permutação dos cilindros de tamanho dois.

Provamos, então, que a relação de equivalência $R_{\Phi}$ é minimal. Então, o odômetro diádico é um homeomorfismo minimal. 


\section{3}

\section{Outra relação de equivalência}

Nesta seção iremos definir outra relação de equivalência no espaço $X$ com o objetivo de relacioná-la com $R_{\Phi}$.

Definição 2.3.1 (Equivalência de caudas). Chamaremos de $R$ a relação em $X$ dada por:

$$
\mathbf{x} \sim_{R} \mathbf{y} \text { se e só se existe } N \geq 0, x_{n}=y_{n} \text { para todo } n>N .
$$

Isto é, $\mathbf{x}$ e $\mathbf{y}$ são equivalentes se diferem apenas em um número finito de entradas.

Proposição 2.3.2. A relação $R$ é uma relação de equivalência.

- Reflexiva: $\mathbf{x} \sim_{R} \mathbf{x}$, já que basta tomar $N=0$;

- Simétrica: se $\mathbf{x} \sim_{R} \mathbf{y}$ então existe $N \geq 0$, tal que $x_{n}=y_{n}$ para todo $n>N$, como a igualdade é simétrica, temos que $\mathbf{y} \sim_{R} \mathbf{x}$;

- Transitiva: sejam $\mathbf{x}, \mathbf{y}, \mathbf{z} \in X$, tais que $\mathbf{x} \sim_{R} \mathbf{y}$ e $\mathbf{y} \sim_{R} \mathbf{z}$. Então existem $N_{1}, N_{2}$ tal que para todo $n>N_{1}, x_{n}=y_{n}$ e para todo $n>N_{2}, y_{n}=z_{n}$. Tomando $N=\max \left\{N_{1}, N_{2}\right\}$, tem-se que $x_{n}=z_{n}$ para todo $n>N$, então $\mathbf{X} \sim_{R} \mathbf{z}$.

Concluímos, portanto, que $R$ é uma relação de equivalência.

Denotaremos a classe de equivalência de $\mathbf{x} \in X$ em relação a $R$ por $\langle\mathbf{x}\rangle_{R}$.

Teorema 2.3.3. Valem as seguintes afirmações:

1. $\langle\mathbf{x}\rangle_{R} \subset\langle\mathbf{x}\rangle_{R_{\Phi}}$.

2. $R_{\Phi}$ é a menor relação de equivalência que contém $R$, isto é, toda classe de equivalência de $R$, está contida em alguma classe de equivalência de $R_{\Phi}$, e tal que $\mathbf{0} \sim \mathbf{1}$.

Prova. Precisaremos do seguinte resultado:

Lema 2.3.4. Sejam $\mathbf{x}, \mathbf{y} \in X$, se $\mathbf{x}$ e $\mathbf{y}$ são iguais menos em um número finito de entradas, então existe $k \in \mathbb{Z}$ tal que $\Phi^{k}(\mathbf{x})=\mathbf{y}$

Prova. Como $\mathbf{x}$ e $\mathbf{y}$ são iguais menos em um número finito de entradas, temos que existe $N>0$ tal que $x_{n}=y_{n}$ para todo $n>N$, consideramos que $N$ é o primeiro número tal que isso ocorre. Tomemos os cilindros $\left[x_{1} \ldots x_{N}\right]$ e $\left[y_{1} \ldots y_{N}\right]$, que são disjuntos. Temos que existe $0<k<2^{N}$ tal que: 


$$
\Phi^{k}\left(\left[x_{1} \ldots x_{N}\right]\right)=\left[y_{1} \ldots y_{N}\right] .
$$

Então $\Phi^{k}(\mathbf{x})=\mathbf{y}^{\prime}$, onde $\mathbf{y}^{\prime}$ está em $\left[y_{1} \ldots y_{N}\right]$. Temos que mostrar que $\mathbf{y}^{\prime}=\mathbf{y}$.

Consideramos o elemento $\left(0^{N} x_{N+1} x_{n+2} \ldots\right) \in[\mathbf{0}]_{N}$. Pela observação 2.2.13, a aplicação $\Phi^{i}\left([\mathbf{0}]_{N}\right)$, onde $0 \leq i \leq 2^{N}-1$ não altera as entradas $0_{\ell}$ para todo $\ell>N$. Temos que existe $k_{1}$ tal que $0 \leq k_{1} \leq 2^{N}-1$ e

$$
\Phi^{k_{1}}\left(\left(0^{N} x_{N+1} x_{N+2} \ldots\right)\right) \in\left[x_{1} \ldots x_{N}\right] .
$$

Então, temos que $\Phi^{k_{1}}\left(\left(0^{N} x_{N+1} x_{N+2} \ldots\right)\right)=\mathbf{x}$. Analogamente, existe $k_{2}$ tal que $0 \leq k_{2} \leq 2^{N}-1 \mathrm{e}$

$$
\Phi^{k_{2}}\left(\left(0^{N} x_{N+1} x_{N+2} \ldots\right)\right) \in\left[y_{1} \ldots y_{N}\right]
$$

Assim,

$$
\Phi^{k_{2}}\left(\left(0^{N} x_{N+1} x_{N+2} \ldots\right)\right)=\left(y_{1} \ldots y_{N} x_{N+1} x_{N+2} \ldots\right)=\mathbf{y} .
$$

Definimos $k=k_{2}-k_{1}$, então

$$
\Phi^{k}(\mathbf{x})=\Phi^{k_{2}-k_{1}}(\mathbf{x})=\Phi^{k_{2}}\left(\Phi^{-k_{1}}(\mathbf{x})\right)=\Phi^{k_{2}}\left(\left(0^{N} x_{N+1} x_{N+2} \ldots\right)\right)=\mathbf{y}
$$

Dessa forma finalizamos a prova do lema.

Continuaremos com a demonstração do teorema. Seja $\mathbf{x} \in X$. Dado $\mathbf{y} \in\langle\mathbf{x}\rangle_{R}$, pelo Lema 2.3.4, obtemos que $\mathbf{y} \in\langle\mathbf{x}\rangle_{\Phi}$, portanto $\langle\mathbf{x}\rangle_{R} \subset\langle\mathbf{x}\rangle_{\Phi}$. Provamos, então o item (1) do teorema.

Falta mostrar o item (2). Como $\Phi(\mathbf{1})=\mathbf{0}$, temos que $\mathbf{0} \sim_{\Phi} \mathbf{1}$. Agora, considere relação de equivalência $S$ tal que $R \subset S \subset R_{\Phi}$ e $\mathbf{0} \sim_{S} \mathbf{1}$. Veremos que $R_{\Phi} \subset S$, isto é que se $\mathbf{x} \sim_{R_{\Phi}} \mathbf{y}$ então $\mathbf{x} \sim_{S} \mathbf{y}$. Considere dois elementos $\mathbf{x}$ e $\mathbf{y}$ tais que $\mathbf{x} \sim_{R_{\Phi}} \mathbf{y}$. Então, existe $k \in \mathbb{Z}$ tal que $y=\Phi^{k}(\mathbf{x})$. Mostraremos que $\mathbf{x} \sim_{S} \Phi^{k}(\mathbf{x})$. Suponha, primeiro, que $\mathbf{x}, \Phi(\mathbf{x}), \Phi^{2}(\mathbf{x}), \ldots, \Phi^{k}(\mathbf{x})$ são todos diferentes de $\mathbf{1}$. Pela definição de odômetro, temos que cada $\Phi^{i}(\mathbf{x})$ difere de $\Phi^{(i+1)}(\mathbf{x})$ em um número finito de entradas para todo $0 \leq i \leq k$, assim temos que $\mathbf{x}$ e $\Phi^{k}(\mathbf{x})$ diferem em um número finito de entradas, então $\mathbf{x} \sim_{R} \Phi^{k}(\mathbf{x})$ e portanto, como $R \subset S, \mathbf{x} \sim_{S} \Phi^{k}(\mathbf{x})$.

Agora, suponha que existe $i \in\{0,1,2, \ldots, k\}$ tal que $\Phi^{i}(\mathbf{x})=\mathbf{1}$, consideremos que esse $i$ é o primeiro número que isso ocorre. Então $\mathbf{x}$ e $\Phi^{i}(\mathbf{x})=\mathbf{1}$ diferem em um número finito de entradas. $\operatorname{Logo} \mathbf{x} \sim_{R} \mathbf{1}$, então $\mathbf{x} \sim_{S} \mathbf{1}$. Como $\Phi(\mathbf{1})=(\mathbf{0})$, temos que se $j$ é tal que $i+1 \leq j \leq k, \Phi^{j}(\mathbf{x})$ considerados dois a dois diferem em um número finito de entradas, assim $\Phi^{k}(\mathbf{x}) \sim_{R} \mathbf{0}$ então $\Phi^{k}(\mathbf{x}) \sim_{S} \mathbf{0}$, como $\mathbf{1} \sim_{S} \mathbf{0}, \mathbf{x} \sim_{S} \mathbf{1}$ e $S$ é relação de equivalência segue que 
$\mathbf{x} \sim{ }_{S} \Phi^{k}(\mathbf{x})$.

Em qualquer um dos casos, mostramos que se $\mathbf{x} \sim_{R_{\Phi}} \mathbf{y}$ então $\mathbf{x} \sim_{S} \mathbf{y}$. Portanto, $R_{\Phi}=S$. Assim, terminamos a demonstração do item (2), e, portanto, a demonstração do teorema. 


\section{3}

\section{Conjuntos de Cantor}

O objetivo deste capítulo é definir o que é um conjunto de Cantor, que na maior parte do tempo será o nosso espaço de trabalho e mostrar que todos os conjuntos de Cantor são homeomorfos.

\section{1}

\section{Conceitos preliminares}

Nesta seção apresentaremos os conceitos necessários para definir um conjunto de Cantor e mostrar que quaisquer dois conjuntos de Cantor são homeomorfos.

Chamaremos de conjuntos clopen, os conjuntos que são simultaneamente abertos e fechados.

Definição 3.1.1 (Conjunto conexo). Um espaço métrico é conexo quando não pode ser decomposto como uma união disjunta de conjuntos abertos e não vazios. Se existe tal decomposição, o espaço métrico é desconexo.

Definição 3.1.2 (Espaço totalmente desconexo). Um espaço métrico é dito totalmente desconexo se seus únicos subconjuntos conexos são pontos ou o conjunto vazio.

Lema 3.1.3. Seja $(X, d)$ um espaço métrico compacto totalmente desconexo e seja $x \in X$ :

1. A interseção de todos os conjuntos clopen que contém $x$ é $\{x\}$;

2. Dado qualquer $r>0$, existe um conjunto clopen $U$ tal que $x \in U \subset$ $B(x, r)$. Isto é, os conjuntos clopen formam uma base para a topologia de $X$.

Prova. Definimos $\mathcal{C}$ como a coleção de todos os conjuntos clopen que contém $x$, $\mathcal{N}$ como a coleção de todos os conjuntos clopen que não contém $x$ e o conjunto.

$$
Y:=\bigcap_{C \in \mathcal{C}} C
$$

Mostraremos, primeiro, que $Y=\{x\}$. Por definição, $\{x\} \in \mathcal{C}, \emptyset \in \mathcal{N}$. Seja um conjunto $R$ que está em $\mathcal{C}$, então $X \backslash R$ não contém x e, portanto, está em $\mathcal{N}$. 
Analogamente, se $R$ está em $\mathcal{N}$, então $X \backslash R$ está em $\mathcal{C}$. Pela definição de $Y$, $x \in Y$ e portanto, $X \backslash Y$ está em $\mathcal{N}$. Como $Y$ é interseção de fechados, então $Y$ é fechado.

Suponha, por absurdo, que $Y$ contém algum ponto além de $x$. Como, por hipótese, $X$ é totalmente desconexo, $Y$ será desconexo. Com isso, existem $U$ e $V$ abertos em $X$ tal que $U \cap Y$ e $V \cap Y$ são disjuntos, não vazios e a união dos dois cobre $Y$.

Suponha que $x \in U$. Como $Y$ é fechado e $X$ é compacto, $Y$ também será compacto. Com isso, podemos tomar $\delta>0$ tal que:

$$
d(y, z) \geq \delta, y \in U \cap Y, z \in V \cap Y .
$$

Definimos o conjunto:

$$
W:=V \cap\left(\bigcup_{y \in V \cap Y} B\left(y, \frac{\delta}{2}\right)\right)
$$

O conjunto $W$ é aberto, já que $V$ e $\bigcup_{y \in V \cap Y} B\left(y, \frac{\delta}{2}\right)$ são abertos.

Afirmação 3.1.4. $W \cap Y=V \cap Y$.

Prova. Seja $y \in W \cap Y$ então $y \in W \subset V$, assim $y \in V \cap Y$. Portanto $W \cap Y \subset V \cap Y$. Falta mostrar que $V \cap Y \subset W \cap Y$. Temos que

$$
W \cap Y=V \cap\left(\bigcup_{y \in V \cap Y} B\left(y, \frac{\delta}{2}\right)\right) \cap Y \supset V \cap Y .
$$

Portanto, $W \cap Y=V \cap Y$. Finalizamos, então, a demonstração da afirmação.

Afirmação 3.1.5. $\bar{W} \cap(U \cap Y)=\emptyset$.

Prova. Seja $w_{n}$ uma sequência em $W$ que converge para $w$. Pela definição do conjunto $W$, para cada $n$, existe $y_{n} \in V \cap Y$, tal que

$$
d\left(y_{n}, w_{n}\right) \leq \frac{\delta}{2}
$$

Dado $u \in U \cap Y$, temos, utilizando a desigualdade triangular, que

$$
\begin{aligned}
d(w, u) \geq d\left(w_{n}, u\right)-d\left(w_{n}, w\right) & \geq d\left(y_{n}, u\right)-d\left(y_{n}, w_{n}\right)-d\left(w_{n}, w\right) \\
& \geq \delta-\frac{\delta}{2}-d\left(w_{n}, w\right) .
\end{aligned}
$$

Então, para todo $n$

$$
d(w, u) \geq \frac{\delta}{2}-d\left(w_{n}, w\right)
$$


Como $w_{n}$ converge para $w$,

$$
\lim _{n \rightarrow \infty} d\left(w_{n}, w\right)=0
$$

Como a equação 3.1.1 vale para todo $n$, temos que $d(w, u) \neq 0$. Então, $w$ não está em $U \cap Y$. Portanto, $\bar{W} \cap(U \cap Y)=\emptyset$.

Usando a afirmação acima temos que

$$
\bar{W} \backslash W \cap(U \cap Y)=\emptyset .
$$

Como, pela Afirmação 3.1.4, $V \cap Y=W \cap Y$, o conjunto $\bar{W} \backslash W$ também é disjunto de $V \cap Y$.

Portanto, $\bar{W} \backslash W$ é um subconjunto fechado de $X \backslash Y$ e como $X$ é compacto, $\bar{W} \backslash W$ também é compacto. Portanto, existe subcoleção finita de elementos de $\mathcal{N}$ que cobrem $\bar{W} \backslash W$, que denotaremos por $N_{1}, N_{2}, \ldots, N_{k}$. Definimos agora o conjunto

$$
Z:=N_{1} \cup \cdots \cup N_{k} \cup W
$$

Como cada $N_{i}$ é aberto e $W$ também, $Z$ é um conjunto aberto.

Agora, veremos que o conjunto $Z$ também é fechado.

$$
\begin{aligned}
\bar{Z} & =\overline{N_{1}} \cup \cdots \cup \overline{N_{k}} \cup \bar{W}=N_{1} \cup \cdots \cup N_{k} \cup \bar{W} \\
& =N_{1} \cup \cdots \cup N_{k} \cup(\bar{W}-W) \cup N_{1} \cup \cdots \cup N_{k} \cup W \subset N_{1} \cup \cdots \cup N_{k} \cup W \\
& \subset Z
\end{aligned}
$$

Portanto, $\bar{Z} \subset Z$, assim $Z$ é um conjunto clopen e não contém $x$. Então, $Z$ é um elemento da coleção $\mathcal{N}$ e portanto deve ser disjunto de $Y$, o que é uma contradição, já que

$$
Z \cap Y=N_{1} \cup \ldots N_{k} \cup W \cap Y \subset W \cap Y \neq \emptyset
$$

Concluímos, então que $Y=\{x\}$.

Falta mostrar que os conjuntos clopen formam uma base para a topologia de $X$.

Para cada $y$ tal que $d(x, y) \geq r$, existe um conjunto clopen $V_{y}$ contendo $y$, mas não contendo $x$. A coleção $\left\{V_{y}\right\}_{y}$ é uma cobertura de $X \backslash B(x, r)$. Como $B(x, r)$ é aberto, o conjunto $X \backslash B(x, r)$ é fechado, como $X$ é compacto, $X \backslash B(x, r)$ também é compacto. Por definição de compacidade, existe uma 
subcobertura finita de $\left\{V_{y}\right\}$, digamos $\left\{V_{y_{1}}, V_{y_{2}}, \ldots, V_{y_{k}}\right\}$. Isto é,

$$
X \backslash B(x, r) \subset \bigcup_{i=1}^{k} V_{Y_{i}},
$$

então,

$$
U:=\bigcap_{i=1}^{k}\left(X \backslash V_{y_{i}}\right) \subset B(x, r) .
$$

Cada $V_{y_{i}}$ é clopen então seus complementos também são, como U é formado por uma interseção de conjuntos clopen, $U$ também é clopen. Além disso, $x$ não está em nenhum $V_{y}$ então $x \in U$. Concluímos, assim, a demonstração do teorema.

Um conceito que também será necessário é o de partição de um espaço métrico.

Definição 3.1.6 (Partição). Seja $(X, d)$ um espaço métrico. Uma partição de $X$ é uma coleção finita de conjuntos clopen que são dois a dois disjuntos e a união é igual a $X$. Dada uma partição $\mathcal{P}$, definimos o seu diâmetro como:

$$
\operatorname{diam}(\mathcal{P})=\max \{\operatorname{diam}(U) \mid U \in \mathcal{P}\}
$$

Dadas duas partições $\mathcal{P}_{1}$ e $\mathcal{P}_{2}$ de $X$. Diz-se que $\mathcal{P}_{2}$ é mais fina que $\mathcal{P}_{1}$, $\left(\mathcal{P}_{1} \succeq \mathcal{P}_{2}\right)$, se todo elemento de $\mathcal{P}_{2}$ está contido em algum elemento de $\mathcal{P}_{1}$.

Definição 3.1.7 (Sequência de partições refinadoras). Seja $(X, d)$ espaço métrico compacto. Uma sequência de partições refinadoras é uma sequência $\left\{\mathcal{P}_{n}\right\}_{n \geq 1}$, tal que:

1. para cada $n, \mathcal{P}_{n}$ é uma partição de $X$;

2. para cada $n, \mathcal{P}_{n} \succeq \mathcal{P}_{n+1}$;

3. $\lim _{n \rightarrow \infty} \operatorname{diam}\left(\mathcal{P}_{n}\right)=0$.

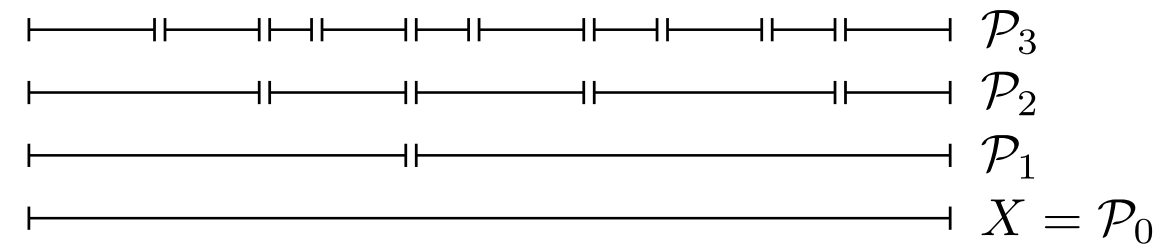

Figura 3.1: Ilustração de uma sequência de partições refinadoras. 
Proposição 3.1.8. Sejam $(X, d)$ um espaço métrico compacto e $\left\{\mathcal{P}_{n}\right\}_{n \geq 1}$ sequência de partições refinadoras, Se $U$ é um subconjunto clopen de $X$ então existe $n$ tal que

$$
U=\bigcup_{\left\{V \in \mathcal{P}_{n} \mid V \cap U \neq \emptyset\right\}} V
$$

Prova. Seja $U$ subconjunto clopen de $X$. Como, por definição de sequência de partições refinadoras, $\operatorname{diam}\left(\mathcal{P}_{n}\right) \rightarrow 0$, existe $n$ tal que $\operatorname{diam}\left(\mathcal{P}_{n}\right)<d(U, X \backslash U)$. Assim, se $V \in \mathcal{P}_{n}$ e $V \cap U \neq \emptyset$, então $V \subset U$. Então

$$
U=\bigcup_{\left\{V \in \mathcal{P}_{n} \mid V \cap U \neq \emptyset\right\}} V
$$

Concluímos a demonstração da proposição.

Definição 3.1 .9 (Ultramétrica). Seja $X$ um conjunto. Uma função $\mathfrak{d}$ : $X \times X \rightarrow[0, \infty)$ é uma ultramétrica se satisfaz as seguintes propriedades:

1. $\mathfrak{d}(x, y)=0$ se e só se $x=y$, para todos $x, y$ em $X$;

2. $\mathfrak{d}(x, y)=\mathfrak{d}(y, x)$, para todos $x, y$ em $X$;

3. $\mathfrak{d}(x, y) \leq \max \{\mathfrak{d}(x, z), \mathfrak{d}(z, y)\}$, para todos $x, y, z$ em $X$.

$O$ conjunto $X$ munido com a ultramétrica $\mathfrak{d}$ será chamado de espaço ultramétrico $(X, d)$.

Observação 3.1.10. Toda ultramétrica é uma métrica.

Prova. É necessário mostrar somente a desigualdade triangular. Dados $x, y, z \in$ $X$ temos que

$$
d(x, y) \leq \max \{d(x, z), d(z, y)\} \leq d(x, z)+d(z, y)
$$

Concluímos, assim, a demonstração da observação.

Definição 3.1.11. Sejam $(X, \mathfrak{d})$ espaço ultramétrico, $x$ em $X$ e $r>0$. Definimos a bola em $(X, \mathfrak{d})$ como:

$$
B_{\mathfrak{d}}(x, r):=\{y \in X \mid \mathfrak{d}(x, y)<r\}
$$

Proposição 3.1.12. Para todo $x$ em $X$ e todo $r>0$ o conjunto $B_{\mathfrak{d}}(x, r)$ é um conjunto aberto. 
Prova. Dados $y$ em $B_{\mathfrak{d}}(x, r)$ e $\delta=\min \{\mathfrak{d}(x, y), r-\mathfrak{d}(x, y)\}$, mostraremos que $B_{\mathfrak{d}}(y, \delta) \subset B_{\mathfrak{d}}(x, r)$. Considere $z$ em $B_{\mathfrak{d}}(y, \delta)$, temos que

$$
\mathfrak{d}(x, z) \leq \max \{\mathfrak{d}(x, y), \mathfrak{d}(y, z)\}=\mathfrak{d}(x, y)<r .
$$

Então $B_{\mathfrak{d}}(y, \delta) \subset B_{\mathfrak{d}}(x, r)$. Concluímos, então, que $B_{\mathfrak{d}}(x, r)$ é um conjunto aberto para todo $x \in X$ e todo $r>0$.

Teorema 3.1.13. Seja $(X, d)$ espaço métrico compacto. As seguintes afirmações são equivalentes:

1. o espaço $(X, d)$ é totalmente desconexo;

2. o espaço $(X, d)$ tem uma sequência de partições refinadoras;

3. existe uma ultramétrica $\mathfrak{d}$ em $X$ que gera a mesma topologia de $d$.

Lema 3.1.14. Seja $(X, \mathfrak{d})$ um espaço ultramétrico, então quaisquer duas bolas de de raio $r>0$ são iguais ou disjuntas.

Prova. Sejam $x, y \in X$ e $r>0$, suponha que

$$
B_{\mathfrak{o}}(x, r) \cap B_{\mathfrak{o}}(y, r) \neq \emptyset
$$

mas que as bolas não sejam iguais. Existem $p \in B_{\mathfrak{d}}(x, r) \cap B_{\mathfrak{d}}(y, r)$ e $q \in B_{\mathfrak{d}}(x, r)$ tal que $q$ não está em $B_{\mathfrak{d}}(y, r)$. Pelo item (3) da definição 3.1.9, temos que

$$
d(y, q) \leq \max \{d(y, p), d(p, q)\} \leq \max \{d(y, p), d(p, x), d(x, q)\} \leq r
$$

então $q \in B_{\mathfrak{o}}(y, r)$, o que é uma contradição.

Corolário 3.1.15. Se X é espaço ultramétrico compacto, dado $r>0$, a coleção $\left\{B_{\mathfrak{d}}(x, r)\right\}_{x \in X}$ é uma partição de $X$.

Prova. Pelo lema acima, a coleção $\left\{B_{\mathfrak{d}}(x, r)\right\}_{x \in X}$ é formada por elementos disjuntos. Além disso, fixe $y \in X$, temos que

$$
B_{\mathfrak{d}}(y, r)=X \backslash \bigcup_{x \in X, x \neq y} B_{\mathfrak{d}}(x, r)
$$

Então, $B_{\mathfrak{d}}(y, r)$ é o complementar de um conjunto aberto e, portanto, fechado. Concluímos que a coleção $\left\{B_{\mathfrak{d}}(x, r)\right\}_{x \in X}$ é uma partição de $X$. 
Continuaremos, neste momento, com a demonstração do Teorema 3.1.13:

$(1) \Rightarrow(2)$ Suponha que $(X, d)$ é totalmente desconexo, construiremos uma sequência de partições refinadoras em $(X . d)$. Definimos $\mathcal{P}_{0}=\{X\}$, temos que $\mathcal{P}_{0}$ é uma partição de $X$, definimos também $r_{0}=\operatorname{diam}(X)$.

Considere uma sequência decrescente $\left(r_{n}\right)_{n \geq 1}$, tal que $r_{n} \rightarrow 0$. Suponha que já construímos, para algum $n \geq 0$, uma partição $\mathcal{P}_{n}$, tal que $\operatorname{diam}(U) \leq r_{n}$ para todo $U$ em $\mathcal{P}_{n}$, construiremos uma partição $\mathcal{P}_{n+1}$, tal que $\operatorname{diam}(U) \leq r_{n+1}$ para todo $U$ em $\mathcal{P}_{n+1}$ e todo elemento de $\mathcal{P}_{n+1}$ está contido em algum elemento de $\mathcal{P}_{n}$, isto é, uma partição mais fina que $\mathcal{P}_{n}$.

Para cada $U$ em $\mathcal{P}_{n}, U$ e $X \backslash U$ são conjuntos clopen em $X$ compacto, portanto $U$ e $X \backslash U$ são compactos. Existe $\delta_{U}>0$, tal que, $d(x, y) \geq \delta_{U}$ para todo $x$ em $U$ e todo $y$ em $X \backslash U$. Definimos:

$$
\delta_{n}=\min \left\{\delta_{U} \mid U \in \mathcal{P}_{n}\right\}
$$

Assim, se $x$ e $y$ estão em elementos diferentes de $\mathcal{P}_{n}$, então $d(x, y) \geq \delta_{n}$.

Pelo Lema 3.1.3, existe um conjunto clopen $U_{x}$ tal que:

$$
x \in U_{x} \subset B\left(x, \min \left\{\delta_{n}, r_{n+1}\right\}\right) .
$$

A coleção $\left\{U_{x}\right\}_{x \in X}$ é uma cobertura de $X$, como $X$ é compacto, existe uma subcobertura finita, digamos $U_{1}, U_{2}, \ldots, U_{N}$, consideraremos que tal subcobertura é minimal, isto é não admite subcobertura própria. Assim, para cada $1 \leq i \leq k$, temos que $U_{i}$ é um conjunto clopen e $\operatorname{diam}\left(U_{i}\right)<\min \left\{\delta_{n}, r_{n+1}\right\}$. Definimos $V_{1}=U_{1}$ e indutivamente $V_{k}=U_{k} \backslash V_{k-1}$ para $k \geq 2$, temos que:

- Para cada $k, V_{k}$ é clopen,

- Como a subcobertura $\left\{U_{i}\right\}_{i=1}^{N}$ é minimal, cada $V_{k}$ é não vazio e está contido em um único elemento da partição $\mathcal{P}_{n}$.

- Assim para cada $V_{k}$, existe $U_{x_{k}}$ em $\mathcal{P}_{n}$, tal que $V_{k} \subset U_{x_{k}}$ então $\operatorname{diam}\left(V_{k}\right) \leq \operatorname{diam}\left(U_{x_{k}}\right) \leq r_{n+1}$.

Portanto, definindo:

$$
\mathcal{P}_{n+1}=\left\{V_{1}, \ldots, V_{N}\right\}
$$

Temos que $\mathcal{P}_{n+1}$ é partição de $X$ mais fina que $\mathcal{P}_{n}$ e que $\operatorname{diam}(V) \leq r_{n+1}$ para todo $V$ de $\mathcal{P}_{n+1}$. Então $\left\{\mathcal{P}_{n}\right\}_{n \geq 0}$ é sequência de partições refinadoras de $(X, d)$. Assim, terminamos a prova de $(1) \Rightarrow(2)$

$(2) \Rightarrow(3)$ Suponha que o espaço $(X, d)$ possui uma sequência de partições refinadoras $\left\{\mathcal{P}_{n}\right\}_{n \geq 0}$. Para cada partição $\mathcal{P}_{n}$, definimos $\delta_{n}$ como fizemos na 
equação (3.1.2). Defina:

$\mathfrak{d}(x, y)=\left\{\begin{array}{l}0, \quad \text { se } x \text { e } y \text { estão no mesmo elemento de } \mathcal{P}_{n} \text { para todo } \mathrm{n}, \\ \delta_{n}, \quad n \text { mínimo tal que } x \text { e } y \text { estão em elementos diferentes de } \mathcal{P}_{n} .\end{array}\right.$

Afirmação 3.1.16. A função d é uma ultramétrica.

Prova. Mostraremos que $\mathfrak{d}$ satisfaz as condições da definição de ultramétrica.

- É claro que $\mathfrak{d}(x, x)=0$. Agora, considere $x, y$ em $X$ tais que $\mathfrak{d}(x, y)=0$. Por definição, $x$ e $y$ estão no mesmo elemento de $\mathcal{P}_{n}$ para todo $n \geq 0$, então

$$
d(x, y) \leq \max \left\{\operatorname{diam}(U) \mid U \in \mathcal{P}_{n}\right\}=\operatorname{diam}\left(\mathcal{P}_{n}\right) \text { para todo } n \geq 0
$$

$\operatorname{Mas}\left\{\mathcal{P}_{n}\right\}_{n \geq 0}$ é sequência de partições refinadoras, logo

$$
\lim _{n \rightarrow \infty} \operatorname{diam}\left(\mathcal{P}_{n}\right)=0
$$

$\operatorname{assim} d(x, y)=0$ e, portanto, $x=y$.

- Também é claro que $\mathfrak{d}(x, y)=\mathfrak{d}(y, x)$.

- Sejam $x, y, z$ em $X$. Se $\mathfrak{d}(x, y)=0$ é claro que

$$
\mathfrak{d}(x, y) \leq \max \{\mathfrak{d}(x, z), \mathfrak{d}(z, y)\}
$$

Considere que $\mathfrak{d}(x, y) \neq 0$, então existe $n \geq 0$ tal que $x$ e $y$ estão em elementos diferentes de $\mathcal{P}_{n}$, consideremos esse $n$ mínimo, então $\mathfrak{d}(x, y)=\delta_{n}$. Temos que $x$ e $z$ ou $y$ e $z$ estão em elementos diferentes de $\mathcal{P}_{n}$. Afirmamos que $x$ e $z$ estão em elementos de $\mathcal{P}_{n}$, então $\mathfrak{d}(x, z) \geq \delta_{n}$, já que $x$ e $z$ podem estar em elementos diferentes de $\mathcal{P}_{i}$, onde $i<n$, e como $\left\{\delta_{n}\right\}_{n \geq 0}$ é uma sequência decrescente, $\delta_{i}>\delta_{n}$. Se $y$ e $z$ não estão em elementos diferentes de $\mathcal{P}_{n}$ então $\max \{\mathfrak{d}(x, z), \mathfrak{d}(y, z)\}=\mathfrak{d}(x, z)$, portanto $\mathfrak{d}(x, y) \leq \max \{\mathfrak{d}(x, z), \mathfrak{d}(z, y)\}$.

Assim, de fato, o é uma ultramétrica.

Falta mostrar que $\mathfrak{d}$ gera a mesma topologia de $d$. Sejam $x$ em $X$ e $r>0$. Como $\delta_{n} \rightarrow 0$, existe $\delta_{n}$, tal que $r>\delta_{n}$. Se $y \notin B(x, r)$ então $d(x, y) \geq r>\delta_{n}$, $\operatorname{assim} x$ e $y$ estão em elementos diferentes de $\mathcal{P}_{n}$ e portanto $\mathfrak{d}(x, y) \geq \delta_{n}$. Logo $y \notin B_{\mathfrak{d}}\left(x, \delta_{n}\right)$. Concluímos que $B_{\mathfrak{d}}\left(x, \delta_{n}\right) \subset B(x, r)$.

Agora, dados $x$ em $X$ e $r>0$, existe $k$ tal que $\delta_{k}<r$. Mostraremos que $B\left(x, \delta_{k}\right) \subset B_{\mathfrak{d}}(x, r)$. Seja $y \in B\left(x, \delta_{k}\right)$, tal que $y \neq x$, então $d(x, y)<\delta_{k}$ e 
portanto $x$ e $y$ estão no mesmo elemento de $\mathcal{P}_{k}$. Como, por hipótese, $\left\{\mathcal{P}_{n}\right\}_{n \geq 0}$ é sequência de partições refinadoras, temos que $x$ e $y$ estão no mesmo elemento de $\mathcal{P}_{i}$ para todo $0 \leq i \leq k, \mathfrak{d}(x, y)=\delta_{l}$, onde $l>k$. Mas a sequência $\left\{\delta_{n}\right\}_{n \geq 0}$ é decrescente, então $\mathfrak{d}(x, y)=\delta_{\ell}<\delta_{k}$, portanto $y \in B_{\mathfrak{d}}\left(x, \delta_{k}\right)$, como $\delta_{k}<r$, temos que $B\left(x, \delta_{k}\right) \subset B_{\mathfrak{v}}(x, r)$. E assim terminamos a prova de $(2) \Rightarrow(3)$.

$(3) \Rightarrow(1)$ : Seja $(X, \mathfrak{d})$ espaço ultramétrico compacto. Mostraremos que todo subconjunto de $X$ com mais de dois pontos é desconexo. Seja $Y \subset X$, tal que $Y$ contém dois pontos distintos, digamos $x$ e $y$. Definimos $r=\frac{\mathfrak{o}(x, y)}{2}$, pelo Lema 3.1.14, temos que $B_{\mathfrak{v}}(x, r)$ e $B_{\mathfrak{v}}(y, r)$ são conjuntos clopen disjuntos em $X$ e além disso são não vazios, portanto $Y$ não é conexo.

Finalizamos, assim, a demonstração do Teorema 3.1.13.

\section{2}

\section{Conjuntos de Cantor}

O conjunto conhecido como conjunto ternário de Cantor, que é formado pelos números no intervalo $[0,1]$ tais que quando expressados em base 3 o dígito 1 não aparece, é um espaço métrico compacto, totalmente desconexo e sem pontos isolados, assim como, o conjunto $\{0,1\}^{\mathbb{N}}$ apresentado no Capítulo 2. Existem mais conjuntos que possuem simultaneamente essas três propriedades e veremos que eles podem ser relacionados.

Definição 3.2.1 (Conjunto de Cantor). Um conjunto de Cantor é qualquer espaço métrico (não vazio), compacto e totalmente desconexo sem pontos isolados.

Dados um conjunto finito $G$ e um espaço métrico $(X, d)$, dizemos que a função $g: G \rightarrow \mathcal{P}(X)$ é subordinada à partição $\mathcal{P}_{n}$ de $X$ se para cada $i$ em $G, g(i)$ é um elemento da partição $\mathcal{P}_{n}$. Para simplificar, usaremos a notação $g_{\mathcal{P}_{n}}: G \rightarrow \mathcal{P}_{n}$. A função $g_{\mathcal{P}_{n}}: U \rightarrow \mathcal{P}_{n}$ é sobrejetiva quando para cada elemento $U$ de $\mathcal{P}_{n}$ existe $i$ em $G$ tal que $g(i)=U$.

Teorema 3.2.2. Quaisquer dois conjuntos de Cantor são homeomorfos.

Prova. Precisaremos de um resultado preliminar.

Lema 3.2.3. Sejam $(X, d)$ espaço métrico compacto sem pontos isolados e $\left\{\mathcal{P}_{n}\right\}_{n \geq 1}$ uma sequência de partições refinadoras de $(X, d)$. Considere $n \geq 1, G$ um conjunto finito e $g_{\mathcal{P}_{n}}: G \rightarrow \mathcal{P}_{n}$ uma sobrejeção subordinada à $\mathcal{P}_{n}$. Então existem $m>n$ e uma função sobrejetora $f: \mathcal{P}_{m} \rightarrow G$ tais que para todo $V$ em $\mathcal{P}_{m}$ temos que $V \subset g_{\mathcal{P}_{n}} \circ f(V)$. 
Prova. Dado $U$ em $\mathcal{P}_{n}$, como $g_{\mathcal{P}_{n}}^{-1}(U) \subset G, g_{\mathcal{P}_{n}}^{-1}(U)$ é um subconjunto finito de $G$. Agora, $U$ é um conjunto infinito, caso fosse finito os elementos de $U$ seriam pontos isolados de $X$. Definimos o conjunto $F_{U}$ como um subconjunto finito de $U$ com mais do que $\# g^{-1}(U)$ elementos. Note que como os elementos da partição são disjuntos, a coleção $\left\{F_{U}\right\}_{U \in \mathcal{P}_{n}}$ é formada por conjuntos dois a dois disjuntos. Definimos o conjunto

$$
F:=\bigcup_{U \in \mathcal{P}_{n}} F_{U}
$$

e $\delta$ como o mínimo da distância entre dois elementos de $F$. Como $\mathcal{P}_{n}$ é uma coleção finita e para cada $U \in \mathcal{P}_{n}, F_{U}$ é um conjunto finito, o conjunto $F$ é finito.

Como $\left\{\mathcal{P}_{n}\right\}_{n \geq 1}$ é uma sequência de partições refinadoras, podemos escolher $m>n$ tal que $\operatorname{diam}\left(\mathcal{P}_{m}\right)<\delta$. Seja $U$ em $\mathcal{P}_{n}$, se tomarmos quaisquer dois elementos de $F_{U}$, a distância entre eles é pelo menos $\delta>\operatorname{diam}\left(\mathcal{P}_{m}\right) \geq \operatorname{diam}(V)$ para todo $V$ em $\mathcal{P}_{m}$. Então quaisquer dois elementos de $F_{U}$ não podem estar no mesmo elemento de $\mathcal{P}_{m}$. Portanto:

$$
\#\left\{V \in \mathcal{P}_{m} \mid V \subset U\right\} \geq \# g_{\mathcal{P}_{n}}^{-1}(U)
$$

Então, qualquer função

$$
f_{U}:\left\{V \in \mathcal{P}_{m} \mid V \subset U\right\} \rightarrow g_{\mathcal{P}_{n}}^{-1}(U)
$$

é uma sobrejeção.

Definimos, a função $f: \mathcal{P}_{m} \rightarrow G$, tal que dado $V$ em $\mathcal{P}_{m}, f(V)=f_{U}(V)$, onde $U$ é o elemento de $\mathcal{P}_{n}$ que contém $V$. A função $f$ é sobrejetora.

Agora, mostraremos que para todo $V$ em $\mathcal{P}_{m}, V \subset g_{\mathcal{P}_{n}} \circ f(V)$. Seja $V$ em $\mathcal{P}_{m}, f(V)=i$, tal que $i$ está em $g_{\mathcal{P}_{n}}^{-1}(U)$ e $U$ contém $V$, portanto:

$$
g_{\mathcal{P}_{n}}(f(V))=g_{\mathcal{P}_{n}}(i)=U \supset V
$$

Então $f$ é a sobrejeção desejada entre $\mathcal{P}_{m}$ e $G$. Concluímos, assim, a demonstração do Lema 3.2.3.

Agora continuaremos a prova do Teorema 3.2.2. Sejam $X$ e $Y$ dois espaços métricos não vazios, compactos, totalmente desconexos e sem pontos isolados. Como os espaços são totalmente desconexos, pelo Teorema 3.1.13 temos que existe uma sequência de partições refinadoras para cada um dos espaços, digamos $\left\{\mathcal{P}_{n}\right\}_{n \geq 1}$ para $X$ e $\left\{\mathcal{Q}_{n}\right\}_{n \geq 1}$ para $Y$. Podemos assumir que $\mathcal{P}_{1}=X$. 
Definiremos indutivamente uma sequência de inteiros crescente $n_{1}<n_{2}<$ ... e funções sobrejetoras:

$$
\begin{gathered}
f_{k}: \mathcal{P}_{n_{k}} \rightarrow \mathcal{Q}_{n_{k-1}} \quad k>1, k \text { impar } \\
g_{k}: \mathcal{Q}_{n_{k}} \rightarrow \mathcal{P}_{n_{k-1}} \quad k>1, k \text { par }
\end{gathered}
$$

tais que

$$
\begin{gathered}
V \subset f_{k} \circ g_{k+1}(V), \quad k \text { ímpar, } V \in \mathcal{Q}_{n_{k+1}}, \\
U \subset g_{k} \circ f_{k+1}(U), \quad k \text { par }, U \in \mathcal{P}_{n_{k+1}} .
\end{gathered}
$$

Começaremos definindo $n_{1}=1$ e $n_{2}=2$. Como a partição $\mathcal{P}_{1}$ possui só um elemento, temos somente uma escolha para definir $g_{2}: \mathcal{Q}_{2} \rightarrow \mathcal{P}_{1}$, que será uma sobrejeção. Usando o Lema 3.2.3, existe $n_{3}>2$ e uma sobrejeção $f_{3}: \mathcal{P}_{n_{3}} \rightarrow \mathcal{Q}_{2}$, tal que $U \subset g_{2} \circ f_{3}(U)$ para todo $U$ em $\mathcal{P}_{n_{3}}$. Agora usando o Lema 3.2.3 para $f_{3}$, existe $n_{4}>n_{3}$ e uma sobrejeção $g_{4}: \mathcal{Q}_{4} \rightarrow \mathcal{P}_{3}$, tal que $V \subset f_{3} \circ g_{4}(V)$ para todo $V$ em $\mathcal{Q}_{n_{4}}$.

Agora, suponha que já definimos as sobrejeções $g_{i-1}, f_{i}$ e $g_{i+1}$ para todo $3 \leq i \leq k$, tal que $k$ é ímpar, onde $V \subset f_{i} \circ g_{i+1}(V)$ para todo $V$ em $\mathcal{Q}_{n_{i+1}}$ e $U \subset g_{i-1} \circ f_{i}(U)$ para todo $U$ em $\mathcal{P}_{n_{i}}$.

Pela hipótese de indução, $g_{k+1}: \mathcal{Q}_{n_{k+1}} \rightarrow \mathcal{P}_{n_{k}}$ é sobrejeção, pelo Lema 3.2.3, existe $n_{k+2}>n_{k+1}$ e sobrejeção $f_{k+2}: \mathcal{P}_{n_{k+2}} \rightarrow \mathcal{Q}_{n_{k-1}}$ tal que $U \subset g_{k+1} \circ f_{k+2}(U)$ para todo $U$ em $\mathcal{P}_{n_{k+2}}$. Usando o Lema 3.2 .3 para a função $f_{k+2}$, existe $n_{k+3}>n_{k+2}$ e sobrejeção $g_{k+3}: \mathcal{Q}_{n_{k+3}} \rightarrow \mathcal{P}_{n_{k+2}}$ tal que $V \subset f_{k+2} \circ g_{k+3}(V)$ para todo $V$ em $\mathcal{Q}_{n_{k+3}}$. Se $k$ é par, a hipótese de indução é que já foram definidas as funções $f_{i-1}, g_{i}$ e $f_{i+1}$ para todo $1 \leq i \leq k$. As funções $g_{k+2}$ e $f_{k+3}$ são definidas de forma análoga.

Definiremos, agora, a função $f: X \rightarrow Y$ ponto a ponto. Fixe $x$ em $X$, explicaremos como iremos definir $f(x)$, para cada $k \geq 1$, denotaremos por $U_{x}^{k}$ o único elemento de $\mathcal{P}_{n_{k}}$ que contém $x$. Como $\left\{\mathcal{P}_{n_{k}}\right\}$ é sequência de partições refinadoras e $\left\{n_{k}\right\}_{k \geq 1}$ é sequência crescente, $U_{x}^{k+2} \subset U_{x}^{k}$ para todo $k \geq 1$. Pela construção que fizemos acima, $g_{k+1} \circ f_{k+2}\left(U_{x}^{k+2}\right)$ está em $P_{n_{k}}$ e contém $U_{x}^{k+2}$. Mas, $U_{x}^{k}$ é o único elemento de $\mathcal{P}_{n_{k}}$ que contém $x$, assim: $g_{k+1} \circ f_{k+2}\left(U_{x}^{k+2}\right)=U_{x}^{k}$. Aplicando $f_{k}$ a ambos os lados obtemos:

$$
f_{k}\left(U_{x}^{k}\right)=f_{k} \circ g_{k+1} \circ f_{k+2}\left(U_{x}^{k+2}\right)=f_{k} \circ\left[g_{k+1}\left(f_{k+2}\left(U_{x}^{k+2}\right)\right)\right] .
$$

Por (3.2.1), sabemos que $f_{k+2}\left(U_{x}^{k+2}\right) \subset f_{k} \circ\left[g_{k+1}\left(f_{k+2}\left(U_{x}^{k+2}\right)\right)\right]$. Então, usando a equação (3.2.3), obtemos que

$$
f_{k+2}\left(U_{x}^{k+2}\right) \subset f_{k}\left(U_{x}^{k}\right)
$$


Portanto, a sequência $\left\{f_{k}\left(U_{x}^{k}\right)\right\}_{k \geq 3}$ de conjuntos clopen é decrescente e, pela definição de $f_{k}, f_{k}\left(U_{x}^{k}\right)$ está em $\mathcal{Q}_{n_{k-1}}$, como o diâmetro da sequência $\left\{\mathcal{Q}_{n_{k}}\right\}$ tende à zero, temos que:

$$
\bigcap_{k \geq 3} f_{k}\left(U_{x}^{k}\right):=\{f(x)\}
$$

Analogamente, podemos definir $g: Y \rightarrow X$ como se segue, dado $y$ em $Y$, para cada $k \geq 1$, denotaremos por $V_{y}^{k}$ o único elemento de $\mathcal{Q}_{n_{k}}$ que contém $y$ e definimos

$$
\bigcap_{k \geq 2} g_{k}\left(V_{y}^{k}\right):=\{g(y)\}
$$

Afirmação 3.2.4. Dados $x$ em $X$ e y em $Y$, temos que $g \circ f(x)=x e$ $f \circ g(y)=y$.

Prova. Dado $k$ par, existe $U_{x}^{k+1}$ em $\mathcal{P}_{n_{k+1}}$ tal que,

$$
\begin{aligned}
f(x) \in f_{k+1}\left(U^{k+1}\right)=V_{f(x)}^{k} & \Rightarrow g(f(x)) \in g_{k}\left(V_{f(x)}^{k}\right)=U_{x}^{k-1} \\
& \Rightarrow g(f(x)) \in \bigcap_{k} U_{x}^{k-1}=\{x\} .
\end{aligned}
$$

Concluímos que $g(f(x))=x$ para todo $x$ em $X$. A demonstração de que para todo $y \in Y, f(g(y))=y$ é análoga.

Podemos afirmar, então que $f$ e $g$ são sobrejeções, já que dados $x$ em $X$ e $y$ em $Y, y$ é a imagem de $g(y)$ por $f$ e $x$ é a imagem de $f(x)$ por $g$.

Afirmação 3.2.5. As funções $f$ e $g$ são injetivas.

Prova. Sejam $x_{1}$ e $x_{2}$ em $X$,

$$
f\left(x_{1}\right)=f\left(x_{2}\right) \Rightarrow g\left(f\left(x_{1}\right)\right)=g\left(f\left(x_{2}\right)\right) \Rightarrow x_{1}=x_{2} .
$$

Então $f$ é injetiva. Mostra-se de forma análoga que $g$ é injetiva.

Portanto, $f$ e $g$ são bijeções e inversas uma da outra.

Para que $f$ e $g$ sejam homeomorfismos falta verificar que as funções $f$ e $g$ são funções contínuas. É suficiente mostrar que $f$ é contínua. Fixe $x$ em $X$. Dados $\epsilon>0$ e $\left\{U_{x}^{k}\right\}_{k}$, existe $k$ ímpar tal que $\operatorname{diam}\left(\mathcal{Q}_{n_{k-1}}\right)<\epsilon$. Então se $z$ está em $U_{x}^{k}, f(z)$ está em $f_{k}\left(U_{x}^{k}\right)$, que contém $f(z)$ e é um está contido em um elemento da partição $\mathcal{Q}_{n_{k-1}}$, portanto, $d_{Y}(f(z), f(y))<\epsilon$.

Concluímos, portanto, que $f$ é um homeomorfismo entre $X$ e $Y$. 


\section{4}

\section{Equivalência orbital}

Neste capítulo, iremos definir o que uma relação de equivalência orbital. Quando for conveniente denotaremos uma relação de equivalência $R$ em um espaço $X$ como o subconjunto

$$
R=\left\{(x, y) \in X \times X \mid x \sim_{R} y\right\} \subset X \times X
$$

Definição 4.0.1 (Conjugação topológica). Considere $\left(X, d_{X}\right)$ e $\left(Y, d_{Y}\right)$ como espaços métricos compactos e $\varphi: X \rightarrow X$ e $\psi: Y \rightarrow Y$ dois homeomorfismos. Uma função $h: X \rightarrow Y$ é dita uma conjugação topológica se é um homeomorfismo e $h \circ \varphi=\psi \circ h$. Se existe tal função $h$, dizemos que $(X, \varphi)$ e $(Y, \psi)$ são conjugados.

Definição 4.0.2. Sejam $R$ e $Q$ relações de equivalência nos espaços métricos $X$ e $Y$, respectivamente. Dizemos que um homeomorfismo $h: X \rightarrow Y$ é uma equivalência entre as relações $R$ e $Q$ se

$$
h\left(\langle x\rangle_{R}\right)=\langle(h(x))\rangle_{Q}
$$

Se $R$ e $Q$ forem relações de equivalência orbitais associadas aos homeomorfismos $\varphi: X \rightarrow X$ e $\psi: Y \rightarrow Y$, respectivamente, dizemos que $h: X \rightarrow Y$ é uma equivalência orbital entre $(X, \varphi)$ e $(Y, \psi)$

Observação 4.0.3. Se h é uma conjugação entre $(X, \varphi)$ e $(Y, \psi)$, então h é uma equivalência orbital.

Prova. Seja $a \in X$ e $b=h(a) \in Y$. Como $h$ é conjugação, temos que dado $n \in Z$

$$
h\left(\varphi^{n}(a)\right)=\psi^{n}(h(a))=\psi^{n}(b) .
$$

Dado $x \in X$, suponha que $b \in h\left(\langle x\rangle_{R_{\varphi}}\right)$. Então, $a \in\langle x\rangle_{R_{\varphi}}$ e portanto existe inteiro $k$ tal que

$$
\varphi^{k}(a)=x .
$$

Então,

$$
h\left(\varphi^{k}(a)\right)=h(x),
$$


$\log O$

$$
h(x)=\psi^{k}(h(a))=\psi^{k}(b) .
$$

Portanto, por definição de $R_{\psi}$, temos que $b \in\langle h(x)\rangle_{R_{\psi}}$. Analogamente, mostramos a inclusão contrária.

Teorema 4.0.4. Sejam $X$ e $Y$ espaços métricos compactos e conexos. Considere $\varphi: X \rightarrow X$ e $\psi: Y \rightarrow Y$ homeomorfismos sem pontos periódicos. Se $h: X \rightarrow Y$ é uma equivalência orbital entre $R_{\varphi}$ e $R_{\psi}$, então o h é uma conjugação entre $(X, \varphi)$ e $(Y, \psi)$ ou é uma conjugação entre $(X, \varphi)$ e $\left(Y, \psi^{-1}\right)$.

Prova. Seja $x \in X$, como $x \sim_{R_{\varphi}} \varphi(x)$ e $h$ é uma equivalência orbital, temos que $h(x) \sim_{R_{\psi}} h(\varphi(x))$. Então, existe inteiro $n$ tal que $h(\varphi(x))=\psi^{n}(h(x))$. Suponha, por absurdo, que existe inteiro $m \neq n$, tal que $h(\varphi(x))=\psi^{m}(h(x))$. Então, $\psi^{m}(h(x))=\psi^{n}(h(x))$ e, portanto, $\psi^{m-n}(h(x))=h(x)$. Como $\psi$ não possui pontos periódicos, temos que $m=n$.

Para cada inteiro $n$, considere o conjunto

$$
X_{n}:=\left\{x \in X \mid h(\varphi(x))=\psi^{n}(h(x))\right\}
$$

Logo a coleação $\left\{X_{n}\right\}_{n \in \mathbb{Z}}$ é formada por conjuntos dois a dois disjuntos e

$$
\bigcup_{n} X_{n}=X
$$

Lema 4.0.5. Para todo $n \in Z$, o conjunto $X_{n}$ é fechado.

Prova. Fixe $n \in Z$ e considere uma sequência $\left\{x_{k}\right\}_{k \geq 0}$ em $X_{n}$ tal que $x_{k}$ converge para um ponto $x$. Para cada $k>0$, temos que

$$
h\left(\varphi\left(x_{k}\right)\right)=\psi^{n}\left(h\left(x_{k}\right)\right) .
$$

Então,

$$
\lim _{k \rightarrow \infty} h\left(\varphi\left(x_{k}\right)\right)=\lim _{k \rightarrow \infty} \psi^{n}\left(h\left(x_{k}\right)\right) .
$$

Como as funções $h, \varphi$ e $\psi$ são contínuas, temos que

$$
h(\varphi(x))=\psi^{n}(h(x))
$$

e, portanto, $x \in X$. Concluímos, assim, que $X_{n}$ é um conjunto fechado.

Usando o lema acima o conjunto $X$ pode ser escrito como união enumerável de conjuntos fechados e dois a dois disjuntos. Como $X$ é espaço métrico compacto e conexo temos que existe $n \in \mathbb{Z}$ tal que $X_{n}=X$. Assim,

$$
h \circ \varphi(x)=\psi^{n} \circ h(x), \quad \text { para todo } x \in X \text {. }
$$


Analogamente, existe $m>0$ tal que

$$
h^{-1} \circ \psi(y)=\varphi^{m} \circ h^{-1}(y), \quad \text { para todo } y \in Y \text {. }
$$

Mostraremos que $n=1$ e $m=1$ ou $n=-1$ e $m=-1$. Dado $x \in X$, temos que $h \circ \varphi(x) \in Y$, portanto

$$
h^{-1} \circ \psi \circ(h \circ \varphi)(x)=\varphi^{m} \circ\left(h^{-1} \circ h\right) \circ \varphi(x) .
$$

Então,

$$
h^{-1} \circ \psi^{n+1} \circ h(x)=\varphi^{m+1} .
$$

Aplicando a função $h$, obtemos que

$$
\psi^{n+1} \circ h(x)=h \circ \varphi^{m+1} .
$$

Logo,

$$
\psi^{n+1} \circ h(x)=\psi^{n(m+1} \circ h(x) .
$$

Como, por hipótese, o homeomorfismo $\psi$ não possui pontos periódicos, temos que $n(m+1)-(n+1)=0$. Então, $n m=1$. Já que $n, m \in \mathbb{Z}$, concluímos que

$$
n=m=1 \quad \text { ou } \quad n=m=-1 .
$$

Finalizamos, então, a demonstração do teorema. 


\section{5 \\ Diagrama de Bratteli}

O objetivo deste capítulo é generalizar a relação de equivalência de caudas $R$ vista no Capítulo 2. Para isto, iremos definir um objeto combinatório representado por um grafo infinito que possui caminhos infinitos formados pelas suas arestas. O espaço de todos estes caminhos, munido de uma métrica apropriada, generaliza o espaço $\{0,1\}^{\mathbf{N}}$ com a métrica considerada no Capítulo 2 .

\section{1}

Diagrama de Bratteli: definição e compacidade do espaço $X_{\mathfrak{B}}$

Definição 5.1.1 (Diagrama de Bratteli). Um diagrama de Bratteli, é uma 4-upla $\mathcal{B}=(V, E, r, s)$, onde $V=\left\{V_{n}\right\}_{n \geq 0}$ e $E=\left\{E_{n}\right\}_{n \geq 1}$ são sequências infinitas de conjuntos finitos, não vazios e dois a dois disjuntos e $s=\left\{s_{n}\right\}_{n \geq 1}$ e $r=\left\{r_{n}\right\}_{n \geq 1}$ são sequências de funções, tais que:

$-V_{0}=\left\{v_{0}\right\}$.

- $s_{n}: E_{n} \rightarrow V_{n-1}$ e $s^{-1}\{v\} \neq \emptyset$ para todo $v \in \cup_{n \geq 1} V_{n}$.

- $r_{n}: E_{n} \rightarrow V_{n}$ e $r^{-1}\{v\} \neq \emptyset$ para todo $v \in \cup_{n \geq 0} V_{n}$.

Os conjuntos $V_{n}$ são chamados de vértices e os conjuntos $E_{n}$ são chamados de arestas. As funções $r_{n}$ e $s_{n}$ são chamadas, respectivamente, de posto e fonte.

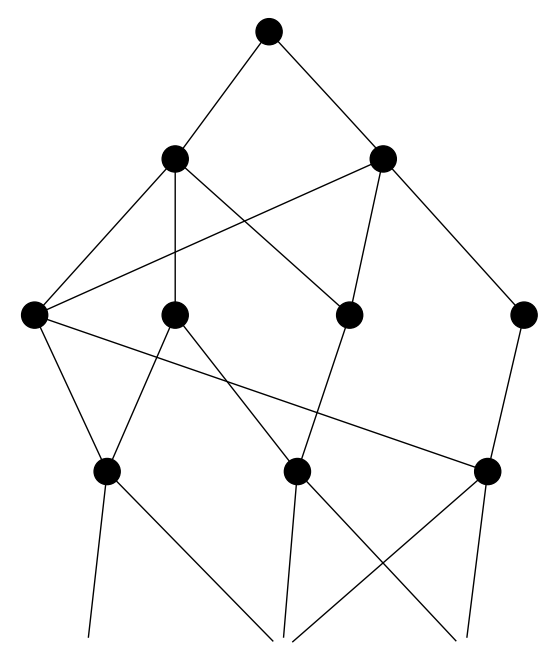

Figura 5.1: Exemplo de um diagrama de Bratteli 
O nosso objetivo é dado um diagrama de Bratteli $\mathfrak{B}$ construir um espaço métrico $X_{\mathfrak{B}}$, compacto, totalmente desconexo e também uma relação de equivalência em $X_{\mathfrak{B}}$.

Definição 5.1.2. Sejam $\mathfrak{B}=(V, E, r, s)$ um diagrama de Bratteli e $n, m \in \mathbb{N}$, tais que $0 \leq m<n$, o conjunto $E_{m, n}$ abaixo é o conjunto de todos os caminhos de $V_{m}$ para $V_{n}$.

$$
E_{m, n}=\left\{\left(p_{m+1}, p_{m+2}, \ldots, p_{n}\right) \mid p_{i} \in E_{i}, r\left(p_{i}\right)=s\left(p_{i+1}\right), m<i \leq n\right\} .
$$

Dados $v \in V_{m}$ e $w \in V_{n}$ denotamos por $E(v, w)$ o conjunto de todos os caminhos de de $v$ até $w$. Definimos o conjunto:

$$
X_{\mathfrak{B}}:=\left\{\left(x_{1}, x_{2}, \ldots\right) \mid x_{n} \in E_{n}, r\left(x_{n}\right)=s\left(x_{n+1}\right), n \geq 1\right\} .
$$

Denotaremos o elemento $\left(x_{1}, x_{2}, \ldots\right)$ de $X_{\mathfrak{B}}$ por $\mathbf{x}$. Quando for conveniente usaremos a notação compacta $\mathbf{x}=\left(x_{i}\right)_{i \geq 1}$.

Note que o conjunto $X_{\mathfrak{B}}$ é formado por todos os caminhos infinitos começando em $v_{0}$.

Também definimos a função $d: X_{\mathfrak{B}} \times X_{\mathfrak{B}} \rightarrow[0, \infty)$ como:

$$
d(\mathbf{x}, \mathbf{y})=\left\{\begin{array}{l}
1, \quad \text { se } x_{1} \neq y_{1}, \\
\inf \left\{2^{-n} \mid x_{i}=y_{i} \text { para todo } 1 \leq i \leq n\right\}
\end{array}\right.
$$

Proposição 5.1.3. O espaço $\left(X_{\mathfrak{B}}, d\right)$ é ultramétrico.

Prova. Mostraremos que a função $d$ satisfaz as condições da definição de ultramétrica.

$-d(\mathbf{x}, \mathbf{y})=0 \Leftrightarrow \inf \left\{2^{-n} \mid x_{i}=y_{i}\right.$ para todo $\left.1 \leq i \leq n\right\}=0 \Leftrightarrow x_{i}=y_{i}$ para todo $i \geq 1 \Leftrightarrow \mathbf{x}=\mathbf{y}$.

- É claro que $d(\mathbf{x}, \mathbf{y})=d(\mathbf{y}, \mathbf{x})$.

- Dados $\mathbf{x}, \mathbf{y}$ e $\mathbf{z}$ em $X_{\mathfrak{B}}$, mostraremos que $d(\mathbf{x}, \mathbf{y}) \leq \max \{d(\mathbf{x}, \mathbf{z}), d(\mathbf{z}, \mathbf{y})\}$. Se $d(\mathbf{x}, \mathbf{z})=1$ ou $d(\mathbf{y}, \mathbf{z})=1$ não há o que fazer. Considere que $d(\mathbf{x}, \mathbf{z})$ e $d(\mathbf{y}, \mathbf{z})$ são ambos diferentes de 1 . Então, há três casos. Ou existem $i \leq j$ positivos tais que $d(\mathbf{x}, \mathbf{z})=\frac{1}{2^{i}}$ e $d(\mathbf{z}, \mathbf{y})=\frac{1}{2^{j}}$, ou uma das distâncias é igual a zero, ou ambas são iguais a zero. Consideremos o caso em que $d(\mathbf{x}, \mathbf{z})=\frac{1}{2^{i}}$ e $d(\mathbf{z}, \mathbf{y})=\frac{1}{2^{j}}$. Neste caso $x_{k}=z_{k}$ para todo $1 \leq k \leq i$, $z_{k}=y_{k}$ para todo $1 \leq k \leq j, x_{i+1} \neq z_{i+1}$ e $y_{j+1} \neq z_{j+1}$, portanto $x_{k}=y_{k}$ para todo $1 \leq k \leq i$ e $x_{i+1} \neq y_{i+1}$. Portanto

$$
d(\mathbf{x}, \mathbf{y})=\frac{1}{2^{i}} \leq \max \{d(\mathbf{x}, \mathbf{z}), d(\mathbf{y}, \mathbf{z})\}
$$


Agora, suponha que $d(\mathbf{x}, \mathbf{z})=0$, então $\mathbf{x}=\mathbf{z}$. Se $d(\mathbf{z}, \mathbf{y})=0$, então $\mathbf{y}=\mathbf{z}$ e portanto $\mathbf{x}=\mathbf{y}$ e $d(\mathbf{x}, \mathbf{y})=0$. Caso $d(\mathbf{y}, \mathbf{z}) \neq 0$, existe $i>0$, tal que $y_{k}=z_{k}=x_{k}$ para todo $1 \leq k \leq i$ e $y_{i+1} \neq z_{i+1}$, logo

$$
d(\mathbf{x}, \mathbf{y})=\frac{1}{2^{i}} \leq \max \{d(\mathbf{x}, \mathbf{z}), d(\mathbf{z}, \mathbf{y})\}
$$

Concluímos a demonstração de que $d$ é uma ultramétrica.

Dados $\mathbf{x} \in X_{\mathfrak{B}}$ e $r>0$, denotaremos por $B(\mathbf{x}, r)$ a bola aberta centrada em $\mathbf{x}$ e de raio $r$.

Definição 5.1.4 (Cilindro). Seja $\mathfrak{B}=(V, E, r, s)$ um diagrama de Bratteli, para qualquer $n \geq 1$ e $p \in E_{0, n}$, tais que $p=\left(p_{1}, \ldots, p_{n}\right)$, definimos o cilindro:

$$
C(p)=\left\{\mathbf{x} \in X_{\mathfrak{B}} \mid\left(x_{1}, \ldots, x_{n}\right)=\left(p_{1}, \ldots, p_{n}\right)\right\} .
$$

Seja $\mathfrak{B}=(V, E, r, s)$ um diagrama de Bratteli. Dado $n>0$ e $p \in E_{0, n}$. Se $e \in E_{n+1}$, definimos

$$
p e:=\left(p_{1}, \ldots, p_{n}, e\right) .
$$

Que chamaremos de concatenação do caminho $p$ com a aresta $e$.

Proposição 5.1.5. Seja $\mathfrak{B}=(V, E, r, s)$ um diagrama de Bratteli. As seguintes afirmações são verdadeiras:

1. Para qualquer $\mathbf{x}$ em $X_{\mathfrak{B}}$ e $n \geq 1$,

$$
B\left(\mathbf{x}, 2^{-n+1}\right)=C\left(x_{1}, \ldots, x_{n}\right)
$$

2. Para qualquer $n \geq 1$ e quaisquer dois caminhos $p$ e q em $E_{0, n}$, temos que $C(p)=C(q)$ se e só se $p=q$, caso contrário são disjuntos.

3. Para todo $n \geq 1$, a coleção $\{C(p)\}_{p \in E_{0, n}}$ é uma partição de $X_{\mathfrak{B}}$, que chamaremos de $\mathcal{P}_{n}$.

4. Dado $n \geq 1$. Para todo $p$ em $E_{0, n}$, temos:

$$
C(p)=\bigcup_{e \in E_{n+1}, s(e)=r(p)} C(p e),
$$

e os conjuntos da união são dois a dois disjuntos.

5. A sequência $\left\{\mathcal{P}_{n}\right\}_{n \geq 0}$ é uma sequência de partições refinadoras para $X_{\mathfrak{B}}$. 
Prova.

1. Dados $\mathbf{x}$ em $X_{\mathfrak{B}}$ e $n \geq 1$. Primeiro, mostraremos, que

$$
B\left(\mathbf{x}, 2^{-n+1}\right) \subset C\left(x_{1}, \ldots, x_{n}\right) .
$$

Seja $\mathbf{y}$ em $B\left(\mathbf{x}, 2^{-n+1}\right)$, temos que

$$
d(\mathbf{x}, \mathbf{y})=\inf \left\{2^{-k} \mid x_{i}=y_{i} \text { para todo } 0 \leq i \leq k\right\}<2^{-n+1},
$$

então $x_{i}=y_{i}$ para todo $0 \leq i \leq k$, onde $k \geq n$, caso contrário $d(\mathbf{x}, \mathbf{y})>2^{-n+1}$, então $\mathbf{y}$ está em $C\left(x_{1}, \ldots, x_{n}\right)$. Falta mostrar que

$$
C\left(x_{1}, \ldots, x_{n}\right) \subset B\left(\mathbf{x}, 2^{-n+1} .\right.
$$

Seja $\mathbf{z}$ em $C\left(x_{1}, \ldots, x_{n}\right)$, por definição, $x_{i}=z_{i}$ para todo $0 \leq i \leq n$, $\operatorname{assim} d(\mathbf{x}, \mathbf{z}) \leq 2^{-n}<2^{-n+1}$, portanto $\mathbf{z}$ está em $B\left(\mathbf{x}, 2^{-n+1}\right)$.

2. Dados $n \geq 1$ e $p, q$ em $E_{0, n}$. Suponha que $C(p)=C(q)$, então $p$ está em $C(q)$, assim, $p=\left(p_{1}, \ldots, p_{n}\right)=q$. Agora, se $p=q$, é direto que $C(p)=C(q)$. Se $p \neq q$, existe $1 \leq i \leq n$ tal que $p_{i} \neq q_{i}$, assim se $\mathbf{x}$ está em $C(p)$ então $\mathbf{x}$ não pode estar em $C(q)$. Da mesma forma, se $\mathbf{x}$ está em $C(q)$ então x não pode estar em $C(p)$. Portanto $C(p)$ e $C(q)$ são disjuntos.

3. É claro que para cada $n \geq 0, X_{\mathfrak{B}}=\cup_{p \in E_{0, n}} C(p)$.

Pelo item 2, a coleção $\{C(p)\}_{p \in E_{0, n}}$ é formada por elementos dois a dois disjuntos.

Pelo item 1, cada elemento de $\{C(p)\}_{p \in E_{0, n}}$ é aberto. Como

$$
\cup_{p \in E_{0, n}} C(p)=X_{\mathfrak{B}}
$$

cada $C(p)$ também será fechado, já que

$$
X \backslash C(p)=\bigcup_{q \in E_{0, n} \cdot q \neq p} C(q) .
$$

Portanto, $C(p)$ é conjunto clopen para todo $p$ em $E_{0, n}$.

Concluímos que $\{C(p)\}_{p \in E_{0, n}}$ é partição de $X_{\mathfrak{B}}$ para todo $n \geq 1$.

4. Dados $n \geq 1$ e $p$ em $E_{n, 0}$. 
Primeiro mostraremos que

$$
C(p) \subset \bigcup_{e \in E_{n+1}, s(e)=r\left(p_{n}\right)} C(p e)
$$

Seja $\mathbf{x}=\left(x_{i}\right)_{i \geq 1}$ em $C(p)$, então $\mathbf{x}$ está em $C\left(p x_{n+1}\right)$, já que $s\left(x_{n+1}\right)=$ $r\left(p_{n}\right)$.

Agora, mostraremos que

$$
\bigcup_{e \in E_{n+1}, s(e)=r\left(p_{n}\right)} C(p e) \subset C(p) .
$$

Seja y um elemento da união. Existe $e$ em $E_{n+1}$, tal que y está em $C(p e)$, então $\left(y_{1}, \ldots, y_{n}, y_{n+1}\right)=p e, \operatorname{assim}\left(y_{1}, \ldots, y_{n}\right)=p$ e portanto $\mathbf{y}$ está em $C(p)$.

Concluímos que

$$
c(p)=\bigcup_{e \in E_{n+1}, s(e)=r\left(p_{n}\right)} C(p e) .
$$

5. Provaremos que $\left\{\mathcal{P}_{n}\right\}_{n \geq 1}$ satisfaz as três condições da definição de sequência de partições refinadoras.

- Pelo item 3, temos que cada $\mathcal{P}_{n}$ é uma partição de $X_{\mathfrak{B}}$.

- Dados $n \geq 1$ e $p$ em $E_{0, n}$, pelo item 4 , cada $C(p)$ em $\mathcal{P}_{n}$ é a união de elementos em $\mathcal{P}_{n+1}$. Então $\mathcal{P}_{n+1}$ é mais fina que $\mathcal{P}_{n}$ para todo $n \geq 1$.

- Dado $n \geq 1$, temos pelo item 1 que $C(p)=B\left(\mathbf{p}, 2^{-n+1}\right)$. Assim

$$
\operatorname{diam}\left(\mathcal{P}_{n}\right)=2^{-n+1} \text { para todo } n \geq 1,
$$

portanto $\lim _{n \rightarrow \infty} \operatorname{diam}\left(\mathcal{P}_{n}\right)=0$.

Finalizamos, então, a demonstração da proposição.

Teorema 5.1.6. Seja $\mathfrak{B}=(V, E, r, s)$ um diagrama de Bratteli, o espaço métrico $\left(X_{\mathfrak{B}}, d\right)$ é compacto.

Prova. O espaço $\left(X_{\mathfrak{B}}, d\right)$ é limitado, já que $X_{\mathfrak{B}} \subset B(\mathbf{x}, 1)$ para todo $\mathbf{x}$ em $X_{\mathfrak{B}}$.

Seja $A \subset X_{\mathfrak{B}}$ um subconjunto infinito, mostraremos que $A$ possui um ponto de acumulação. Temos que

$$
A=\bigcup_{e \in E_{1}} A \cap C(e)
$$


O conjunto $E_{1}$ é finito, como $A$ é infinito, um dos elementos da união tem que ser infinito. Assim existe $e_{1} \in E_{1}$, tal que $A \cap C\left(e_{1}\right)$ é infinito. Suponha, indutivamente, que para $n$, existe $e_{n}$ em $E_{n}$, tal que $A \cap C\left(e_{1}, \ldots, e_{n}\right)$ é um conjunto infinito. Sabemos pelo item (4) da Proposição 5.1.5 que:

$$
A \cap C\left(e_{1}, \ldots, e_{n}\right)=\bigcup_{e \in E_{n+1}, s(e)=r\left(e_{n-1}\right)} A \cap C\left(e_{1}, \ldots, e_{n}, e\right)
$$

Como, $A \cap C\left(e_{1}, \ldots, e_{n}\right)$ é um conjunto infinito pela hipótese de indução, existe $e_{n+1}$ em $E_{n+1}$ tal que $A \cap C\left(e_{1}, \ldots, e_{n}, e_{n+1}\right)$ é infinito.

Definimos, indutivamente, $\mathbf{x}=\left(e_{1}, e_{2}, \ldots\right)$, temos que $\mathbf{x}$ está em $X_{\mathfrak{B}}$. Dados $r>0$ e $n \geq 1$, tais que $2^{-n+1}<r$, temos que:

$$
B(x, r) \cap A \supset B\left(x, 2^{-n+1}\right) .
$$

Usando o item 1 da Proposição 5.1.5, temos:

$$
B\left(x, 2^{-n+1}\right) \cap A=C\left(e_{1}, \ldots, e_{n}\right) \cap A,
$$

assim

$$
C\left(e_{1}, \ldots, e_{n}\right) \cap A \subset B(x, r) \cap A .
$$

Como $A \cap C\left(e_{1}, \ldots, e_{n}\right)$ é um conjunto infinito para todo $n \geq 1$, então $B(x, r) \cap A$ também é um conjunto infinito, portanto $B(x, r) \cap A \backslash\{x\}$ é um conjunto não vazio. Concluímos então que $x$ é um ponto de acumulação de $A \subset X_{\mathfrak{B}}, \operatorname{assim}\left(X_{\mathfrak{B}}, d\right)$ é espaço métrico compacto.

Lema 5.1.7. Dados um diagrama de Bratteli $\mathfrak{B}=(V, E, r, s)$ e $m, n \geq 0$, tais que $n>m$, temos que para todo $w$ em $V_{n}$ existe $v$ em $V_{m}$ ligado a $w$ por um caminho em $E_{m, n}$.

Prova. Como $r^{-1}\{w\} \neq \emptyset$ para todo $w$ em $V_{n}$ para todo $n \geq 1$, existe $v_{n-1}$ em $V_{n-1}$ ligado a $w$ por algum caminho em $E_{n-1, n}$. Indutivamente e concatenando os caminhos, existe $v$ em $V_{m}$ ligado a $w$ por algum caminho em $E_{m, n}$.

Teorema 5.1.8. Dado um diagrama de Bratteli $\mathfrak{B}=(V, E, r, s)$. Suponha que $F$ é um subconjunto infinito de E, tal que

$$
s(F) \subset r(F) \cup\left\{v_{0}\right\} .
$$

Então F contém um caminho infinito. 
Prova. Seja $F$ um subconjunto infinito de $E$ que satisfaça as hipóteses do teorema, isto é, para todo e em $F \backslash E_{1}$ existe aresta $u(e)$ em $F$ tal que $s(e)=r(u(e))$. Observe que $s\left(E_{1}\right)=\left\{v_{0}\right\}$.

Lema 5.1.9. Dado $n \geq 1$, definimos o conjunto

$$
F_{0, n}=\left\{\left(f_{1}, f_{2}, \ldots, f_{n}\right) \in E_{0, n} \mid f_{i} \in F \text { para todo } 1 \leq i \leq n\right\}
$$

Temos que $F_{0, n}$ é não vazio.

Prova. Como cada $E_{i}$ é um conjunto finito e, por hipótese, $F$ é infinito, existe um elemento em $F$ que não está em $E_{1} \cup \cdots \cup E_{n-1}$, digamos que esteja em $E_{m}$. Denotaremos tal elemento por $e_{m}$. Temos que $m \geq n$.

Por hipótese, existe em $F$ aresta em $E_{m-1}$, digamos $e_{m-1}$, tal que $s\left(e_{m}\right)=r\left(e_{m-1}\right)$. Indutivamente, para cada $1 \leq k \leq n-1$, existe em $F$ uma aresta $e_{m-k}$ em $E_{m-k}$ tal que $s\left(e_{m-k+1}\right)=r\left(e_{m-k}\right)$. Então o caminho $\left(e_{1}, e_{2}, \ldots, e_{m}\right)$ está em $F$ e como $m \geq n$, o caminho $\left(e_{1}, \ldots, e_{n}\right)$ está em $F_{0, n}$. Provando o lema.

Voltamos, agora, para a demonstração do Teorema 5.1.8. Agora, para cada $n \geq 1$, definimos

$$
X_{n}:=\left\{\mathbf{x} \in X_{\mathfrak{B}} \mid\left(x_{1}, x_{2}, \ldots, x_{n}\right) \in F_{0, n}\right\}=\bigcup_{p \in F_{0, n}} C(p) .
$$

Temos que, para cada $n \geq 1$

1. $X_{n}$ é não vazio, já que $F_{0, n}$ é não vazio.

2. $X_{n}$ é clopen, já que cada $C(p)$ é clopen e existe um número finito de elementos em $F_{0, n}$.

3. $X_{n+1} \subset X_{n}$.

Como $X_{\mathfrak{B}}$ é compacto, as condições (1), (2), (3), implicam que $\bigcap_{n \geq 1} X_{n}$ é não vazio. Pela definição do conjunto $X_{n}$, temos que qualquer elemento de $\bigcap_{n \geq 1} X_{n}$ é um caminho infinito e está em $F$. 


\section{2}

\section{Propriedade telescópica de um diagrama de Bratteli}

Agora, apresentaremos a noção de passar a uma subsequência em um diagrama de Bratteli, que será chamada de propriedade telescópica de uma diagrama de Bratteli.

Definição 5.2.1. Dados $\mathfrak{B}=(V, E, r, s)$ um diagrama de Bratteli e uma sequência estritamente crescente de inteiros $m_{0}=0<m_{1}<m_{2}<\ldots$. Definimos o diagrama de Bratteli $\mathfrak{T}=(W, F, \bar{r}, \bar{s})$ obtido a partir de $\mathfrak{B}$ através da subsequência $\left\{m_{k}\right\}_{k \in \mathbb{N}}$,como se segue:

$-W_{n}=V_{m_{n}} ;$

- $F_{n}=E_{m_{n-1}, m_{n}}$

$-\bar{s}=\left\{\bar{s}_{n}\right\}_{n \geq 1}$, onde $\bar{s}: F_{n}=E_{m_{n-1}, m_{n}} \rightarrow W_{n-1}=V_{m_{n-1}}$, para todo $n \geq 1$, tal que $\bar{s}^{-1}\{v\}$ é não vazio para todo $v$ em $\cup_{n \geq 0} F_{n}$;

$-\bar{r}=\left\{r_{n}\right\}_{n \geq 1}$, onde $\bar{r}: F_{n}=E_{m_{n-1}, m_{n}} \rightarrow W_{n}=V_{m_{n}}$, para todo $n \geq 1$, tal que $\bar{r}^{-1}\{v\}$ é não vazio para todo $v$ em $\cup_{n \geq 1} F_{n}$.

Assim, dado

$$
\mathbf{x}=\left(x_{1}, x_{2}, \ldots\right)
$$

em $X_{\mathfrak{B}}$, o caminho

$$
\overline{\mathbf{x}}=\left(\left(x_{1}, \ldots, x_{m_{1}}\right),\left(x_{m_{1}+1}, \ldots, x_{m_{2}}\right), \ldots\right)
$$

está em $X_{\mathfrak{T}}$. Definimos, então, a função de blocos

$$
f=f_{\mathfrak{B}, \mathfrak{T}}: X_{\mathfrak{B}} \rightarrow X_{\mathfrak{T}},
$$

tal que

$$
f\left(x_{1}, x_{2}, \ldots\right)=\left(\left(x_{1}, \ldots, x_{m_{1}}\right),\left(x_{m_{1}+1}, \ldots, x_{m_{2}}\right), \ldots\right) .
$$

Proposição 5.2.2. Se $\mathfrak{T}=(W, F, \bar{r}, \bar{s})$ é obtido a partir de $\mathfrak{B}=(V, F, r, s)$ através de uma subsequência $\left\{m_{k}\right\}_{k \geq 1}$ então a função de blocos

$$
f=f_{\mathfrak{B}, \mathfrak{T}}: X_{\mathfrak{B}} \rightarrow X_{\mathfrak{T}}
$$

é um homeomorfismo.

Prova. Como $X_{\mathfrak{B}}$ e $X_{\mathfrak{T}}$ são espaços métricos compactos, basta mostrar que $f$ é uma bijeção contínua. Claramente, a função $f$ é injetiva e sobrejetiva. Agora, 
iremos mostrar que $f$ é contínua. Dado $\epsilon>0$, existe $r$ em $\mathbb{N}$, tal que $\frac{1}{2^{r}}<\epsilon$. Seja $i$ em $\mathbb{N}$, tal que

$$
m_{r}<i \leq m_{r+1} .
$$

Dado $\mathbf{x}$ em $X_{\mathfrak{B}}$. Se $\mathbf{y}$ em $X_{\mathfrak{B}}$ é tal que $d(\mathbf{x}, \mathbf{y})<\frac{1}{2^{i}}$ então $d(f(\mathbf{x}), f(\mathbf{y}))<\frac{1}{2^{r}}<\epsilon$. Então $f$ é contínua. Concluímos que $f$ é um homeomorfismo.

\section{3}

\section{Relação de equivalência AF}

Já mostramos que o espaço $\left(X_{\mathfrak{B}}, d\right)$ é um espaço ultramétrico e compacto. Agora, definiremos uma relação de equivalência neste espaço.

Definição 5.3.1 (Relação-AF). Seja $\mathfrak{B}=(V, E, r, s)$ um diagrama de Bratteli. Chamaremos $R_{\mathfrak{B}}$ a relação em $X_{\mathfrak{B}}$ dada por:

$\mathbf{x} \sim_{\mathfrak{B}} \mathbf{y}$ se e só se $\mathbf{x}$ e $\mathbf{y}$ são iguais menos em um número finito de entradas.

Em particular, existe $i_{0}$ tal que $x_{i}=y_{i}$ para todo $i>i_{0}$.

A sigla AF significa aproximadamente finita.

Proposição 5.3.2. A relação- $A F$ é uma relação de equivalência.

Prova. Mostraremos que a relação-AF satisfaz as propriedades:

- Reflexiva: É claro que $\mathbf{x} \sim_{\mathfrak{B}} \mathbf{x}$.

- Simétrica: Também é claro que se $\mathbf{x} \sim_{\mathfrak{B}} \mathbf{y}$ então $\mathbf{y} \sim_{\mathfrak{B}} \mathbf{x}$.

- Transitiva: Dados $\mathbf{x}, \mathbf{y}$ e $\mathbf{z}$ em $X_{\mathfrak{B}}$ tais que $\mathbf{x} \sim_{\mathfrak{B}} \mathbf{y}$ e $\mathbf{y} \sim_{\mathfrak{B}} \mathbf{z}$. Como $\mathbf{x}$ difere de $\mathbf{y}$ em um número finito de entradas e $\mathbf{y}$ difere em um número finito de entradas de $\mathbf{z}$ então $\mathbf{x}$ e $\mathbf{z}$ também diferem em um número finito de entradas, assim $\mathbf{x} \sim_{\mathfrak{B}} \mathbf{z}$.

Denotaremos a classe de equivalência de um elemento $\mathbf{x}$ em $X_{\mathfrak{B}}$ por $\langle\mathbf{x}\rangle_{R_{\mathfrak{B}}}$. A proposição abaixo apresenta o efeito de passar a uma subsequência na relação-AF.

Proposição 5.3.3. Dado $\mathfrak{B}=(V, E, r, s)$ um diagrama de Bratteli $e$ o diagrama $\mathfrak{T}=(W, F, \bar{r}, \bar{s})$ obtido a partir de $\mathfrak{B}$ através de uma subsequência $m_{0}=0<m_{1}<\ldots$ Seja $f$ o homeomorfismo da Proposição 5.2.2. Então $f\left(\langle\mathbf{x}\rangle_{R_{\mathfrak{B}}}\right)=\langle f(\mathbf{x})\rangle_{R_{\mathfrak{T}}}$. 
Prova. Primeiro mostraremos que dado $\mathrm{x}$ em $X_{\mathfrak{B}}$,

$$
f\left(\langle\mathbf{x}\rangle_{R_{\mathfrak{B}}}\right) \subset\langle f(\mathbf{x})\rangle_{R_{\mathfrak{x}}}
$$

Seja $\mathbf{y}$ em $f\left(\langle\mathbf{x}\rangle_{R_{\mathfrak{B}}}\right)$, então existe $\mathbf{z}$ em $\langle\mathbf{x}\rangle_{R_{\mathfrak{B}}}$, tal que $f(\mathbf{z})=\mathbf{y}$. Temos que $\mathbf{y}$ está em $\langle f(\mathbf{x})\rangle_{R_{\mathfrak{x}}}$. Suponha por absurdo que isso não aconteça. Como $f(\mathbf{z})=\mathbf{y}$, $\mathbf{z}$ e $\mathbf{y}$ iriam ter um número diferentes de entradas, o que é uma contradição.

Agora, provaremos que

$$
\langle f(\mathbf{x})\rangle_{R_{\mathfrak{T}}} \subset f\left(\langle\mathbf{x}\rangle_{R_{\mathfrak{B}}}\right) .
$$

Seja $\mathbf{y}$ em $\langle f(\mathbf{x})\rangle_{R_{\mathfrak{x}}}$. Como $f$ é sobrejeção, existe $\mathbf{z}$ em $X_{\mathfrak{B}}$ tal que

$$
f(\mathbf{z})=\left(\left(z_{1}, \ldots, z_{m_{1}}\right),\left(z_{m_{1}+1}, \ldots, z_{m_{2}}\right) \ldots\right)=\mathbf{y}
$$

Como y está em $\langle f(\mathbf{x})\rangle_{R_{\mathfrak{T}}}, \mathbf{y}$ e $f(\mathbf{x})=\left(\left(x_{1}, \ldots, x_{m_{1}}\right),\left(x_{m_{1}+1}, \ldots, x_{m_{2}}\right) \ldots\right)$ diferem em um número finito de entradas, então $\mathbf{z} \sim \mathbf{x}$, assim $\mathbf{y}=f(\mathbf{z}) \in f\left(\langle\mathbf{x}\rangle_{R_{\mathfrak{B}}}\right)$. Concluímos, assim, a demonstração da proposição.

Pela Proposição 5.3.3, as relações de equivalência $R_{\mathfrak{B}}$ e $R_{\mathfrak{T}}$ são equivalentes.

Definição 5.3.4. Dizemos que um diagrama de Bratteli $\mathfrak{B}=(V, E, r, s)$ possui todas as arestas conectadas quando para todo vértice $v$ em $V_{n-1}$ e todo $w$ em $V_{n}$ existe uma aresta e em $E_{n}$, tal que $s(e)=v$ e $r(e)=w$.

Teorema 5.3.5. Seja $\mathfrak{B}=(V, E, r, s)$ um diagrama de Bratteli. As seguintes afirmações são equivalentes:

1. Para cada $m \geq 0$ e cada vértice $v$ em $V_{m}$, existe $n(v)>m$ tal que para todo $w$ em $V_{n}$ existe um caminho de $v$ para $w$.

2. Para cada $m \geq 0$, existe $n(m)>m$ tal que para todo $v$ em $V_{m}$ e para todo $w$ em $V_{n}$ existe um caminho de $v$ para $w$.

3. Existe um diagrama de Bratteli $\mathfrak{T}=(W, F, \bar{r}, \bar{s})$ obtido a partir de $\mathfrak{B}$ através de uma subsequência, que possui todas as arestas conectadas.

4. A relação de equivalência $R_{\mathfrak{B}}$ é minimal.

Prova. Mostraremos que as afirmações são equivalentes.

$(1) \Rightarrow(2)$ : Dados $m \geq 0$ e $v$ em $V_{m}$, existe $n(v)>m$, tal que para todo $w$ em $V_{n(v)}$, existe um caminho de $v$ para $w$. Definimos

$$
n:=\max \left\{n(v) \mid v \in V_{m}\right\} .
$$


Então, pelo Lema 5.1.7, para todo $v$ em $V_{m}$ e todo $w$ em $V_{n}$, existe um caminho de $v$ para $w$.

$(2) \Rightarrow(3)$ : Seja $m_{0}=0$, existe $m_{1}>0$ tal que, para todo $v$ em $V_{m_{1}}$ existe um caminho entre $v_{0}$ e $v$. Indutivamente, para cada $m_{i}$, existe $m_{i+1}>m_{i}$, tal que para todo $v$ em $V_{m_{i}}$ e todo $w$ em $V_{m_{i+1}}$, existe um caminho entre $v$ e $w$.

Definimos então a sequência crescente $m_{0}<m_{1}<m_{2}<\ldots$ e obtemos o diagrama $\mathfrak{T}$ a partir de $\mathfrak{B}$ através da subsequência $\left\{m_{k}\right\}_{k \geq 0}$.

Falta mostrar que $\mathfrak{T}$ possui todas as arestas conectadas. Seja $v$ em $W_{n-1}$, então $v$ está em $V_{m_{n-1}}$. Por hipótese, existe um caminho $e(v, w)$ entre todo $v$ em $V_{m_{n-1}}=W_{n-1}$ e todo $w$ em $V_{m_{n}}=W_{n}$. Como $e(w, v)$ liga $v$ a $w$, temos que $\bar{s}(e(v, w))=v$ e $\bar{r}(e(v, w))=w$.

$(3) \Rightarrow(4)$ : Seja $\mathfrak{T}$ o diagrama de Bratteli obtido a partir $\mathfrak{B}$ por uma subsequência $\left\{m_{k}\right\}_{k \geq 0}$. Dados $\mathbf{x}$ e $\mathbf{y}$ em $X_{\mathfrak{B}}$ é suficiente mostrar que

$$
C\left(y_{1}, \ldots, y_{m_{1}}\right) \cap\langle\mathbf{x}\rangle_{R_{\mathfrak{B}}} \neq \emptyset .
$$

Definimos $v_{y}=r\left(y_{m_{1}}\right)$. Dado $w=s^{-1}\left(x_{m_{2}+1}\right)$ em $W_{2}=V_{m_{2}}$, existe $e\left(v_{y}, w\right)$ aresta em $F_{n}=E_{m_{1}, m_{2}}$ ligando $v_{y}$ a $w$, já que $\mathfrak{T}$ possui todas as arestas conectadas. Assim,

$$
\left(y_{1}, \ldots, y_{n}, e\left(v_{y}, w\right), x_{m_{2}+1}, \ldots\right) \sim_{\mathfrak{B}} \mathbf{x}
$$

Além disso, $\left(y_{1}, \ldots, y_{n}, e\left(v_{y}, w\right), x_{m_{2}+1}, \ldots\right)$ está em $C\left(y_{1}, \ldots, y_{m_{1}}\right)$. Portanto,

$$
C\left(y_{1}, \ldots, y_{m_{1}}\right) \cap\langle\mathbf{x}\rangle_{R_{\mathfrak{B}}} \neq \emptyset .
$$

$(4) \Rightarrow(1)$ : Suponha, por absurdo, que existam $m \geq 0$ e $v$ em $V_{m}$ tal que para todo $m+k>m$ existe $w$ em $V_{m+k}$ que não é ligado a $v$ por nenhum caminho. Construiremos, indutivamente, um ponto $\mathbf{x}$ em $X_{\mathfrak{B}}$ e uma sequência $\left\{\bar{y}_{k}\right\}_{k \geq 1}$, onde cada elemento $\bar{y}_{k}$ está em $E_{m, m+k}$ e é disjunto do caminho $\left(x_{m+1}, \ldots, x_{m+k}\right)$. Faremos a prova por indução em $k$.

- Caso $k=1$ : Existe $w_{1}$ em $W_{m+1}$ que não é ligado a a $v$ por nenhum caminho em $E_{m, m+1}$. Definimos, então $x_{m+1}$ como algum elemento de $s^{-1}(v)$ e $\bar{y}_{1}$ como alguma aresta do conjunto $r^{-1}\left(w_{1}\right)$. Por hipótese, $s^{-1}(v)$ e $r^{-1}\left(w_{1}\right)$ são disjuntos, pois caso contrário haveria um caminho ligando $v$ a $w_{1}$, o que é uma contradição. Então $x_{m+1}$ e $\bar{y}_{1}$ são disjuntos.

- Suponha que já construímos, para todo $1 \leq i \leq k$, as arestas $x_{m+i} \in E_{m+i}$ e a concatenação $\left(x_{m+1}, \ldots, x_{m+i}\right) \in E_{m, m+i}$ e o caminho $\bar{y}_{i}$, de forma que $\left(x_{m+1}, \ldots, x_{m+i}\right)$ e $\bar{y}_{i}$ são disjuntos. 
- Construiremos o elemento $x_{m+k+1} \in E_{m+k+1}$ e $\bar{y}_{k+1}$. Temos que existe $w_{k+1}$ em $V_{m+k+1}$ que não é ligado a $v$ por nenhum caminho. Definimos então $x_{m+k+1}$ como algum elemento de $s^{-1}\left(r\left(x_{m+k}\right)\right)$.

Definimos $\bar{y}_{k+1}$ como algum caminho em $E_{m, m+k+1}$, tal que $y_{m+k+1} \in$ $r^{-1}\left(w_{m+k+1}\right)$. Os caminhos $\left(x_{m+1}, \ldots, x_{m+k+1}\right)$ e $\bar{y}_{k+1}$ são disjuntos, já que $w_{m+k+1}$ não é ligado a $v$ por nenhum caminho.

Seja $\left(x_{1}, x_{2}, \ldots, x_{m}\right)$ algum caminho entre $v_{0}$ e $v$, definimos

$$
\mathbf{x}=\left(x_{1}, \ldots, x_{m}, x_{m+1}, \ldots, x_{m+k}, \ldots\right)
$$

e a sequência $\left\{\mathbf{y}_{k}\right\}_{k \geq 1}$, tal que para cada $k, \mathbf{y}_{k}$ é um elemento de $X_{\mathfrak{B}}$ que contém o caminho $\bar{y}_{k}$ definido acima. Como, pelo Teorema 5.1.6, $X_{\mathfrak{B}}$ é compacto, temos que a sequência $\left\{\mathbf{y}_{k}\right\}_{k \geq 1}$ possui subsequência convergente, digamos que para o ponto $\mathbf{y}$. Repare que y não está em $\langle\mathbf{x}\rangle_{R_{\mathfrak{B}}}$, o que é uma contradição, já que $R_{\mathfrak{B}}$ é minimal.

Definição 5.3.6 (Diagrama de Bratteli simples). Se um diagrama de Bratteli satisfaz quaisquer uma das três primeiras condições do Teorema 5.3.5, dizemos que ele é simples.

\section{4}

\section{Exemplos}

Considere o diagrama de Bratteli $\mathfrak{B}$ apresentado na Figura 5.2. O conjunto $X_{\mathfrak{B}}$ representa o espaço $\{0,1\}^{\mathbb{N}}$. Neste caso a relação $R_{\mathfrak{B}}$ é a relação de equivalência de caudas apresentada na Definição 2.3.1.

Temos que $\mathcal{B}$ satisfaz a condição 1 do Teorema 5.3.5, portanto a relação de equivalência $R_{\mathcal{B}}$ é minimal. 


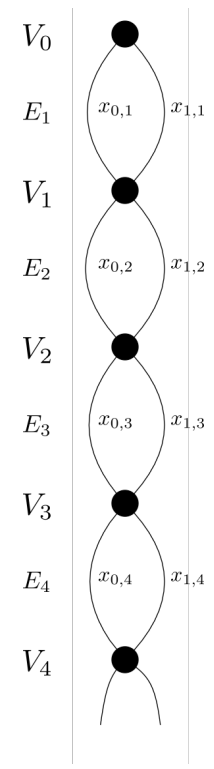

Figura 5.2: Diagrama de Bratteli que representa $\{0,1\}^{\mathbb{N}}$

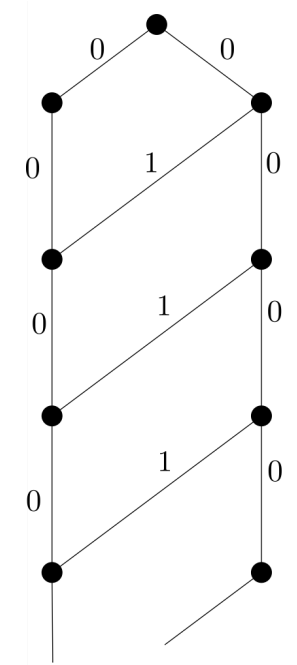

Figura 5.3: Diagrama $\mathfrak{T}$, onde $X_{\mathfrak{T}}$ é um subconjunto de $\{0,1\}^{\mathbb{N}}$.

Agora, considere o diagrama de Bratteli $\mathfrak{T}$ da Figura 5.3. Note que o espaço $X_{\mathfrak{T}}$ é formado pelas sequências infinitas $\mathbf{x}=\left(x_{n}\right)_{n \geq 1}$, tais que $x_{n}=0$ para todo $n \geq 1$ ou existe $i \geq 1$ tal que $x_{i}=1$ e $x_{j}=0$ para todo $i \neq j$. Considere o conjunto

$$
X=\left\{\frac{1}{2^{i}} \mid i \geq 0\right\}
$$

Existe um homeomorfismo natural entre $X_{\mathfrak{T}}$ e $X$.

Seja o vértice $v_{1,1}$. Para todo $i>1$, não há caminho que ligue $v_{1,1}$ ao vértice $v_{2, i}$. Logo, o diagrama $\mathfrak{T}$ não satisfaz a condição 1 do Teorema 5.3 .5 e, portanto, a relação $R_{\mathfrak{T}}$ não é minimal. 


\section{6}

\section{O modelo de Bratteli-Vershik}

No capítulo anterior, definimos um diagrama de Bratteli $\mathfrak{B}$ e a este diagrama associamos o espaço $X_{\mathfrak{B}}$. Definimos a relação de equivalência $R_{\mathfrak{B}}$ que é uma generalização da relação $R$ definida no Capítulo 2. No Capítulo 2 também definimos a relação $R_{\varphi}$. Agora, o objetivo é definir uma relação de equivalência no espaço $X_{\mathfrak{B}}$ que generalize a relação $R_{\varphi}$.

\section{1}

\section{Diagrama de Bratteli ordenado}

Definição 6.1.1 (Ordem parcial). Dado um conjunto $X$, uma relação $\geq$ é dita uma ordem parcial se satisfaz as seguintes propriedades:

- Reflexiva: dado $x \in X, x \geq x$.

- Antissimétrica: Dados $x, y \in X$, se $x \geq y$ e $y \geq x$, então $x=y$.

- Transitiva: Sejam $x, y, z \in X$. Se $x \geq y$ e $y \geq z$ então $x \geq z$.

Definição 6.1.2 (Diagrama de Bratteli ordenado). Um diagrama de Bratteli ordenado é formado por um diagrama de Bratteli $\mathfrak{B}=(V, E, r, s)$ e uma ordem parcial $\geq$ em $E$, tal que duas arestas $e_{1}$ e $e_{2}$ podem ser comparadas se e só se $r\left(e_{1}\right)=r\left(e_{2}\right)$. Denotaremos tal diagrama por $\mathfrak{B}=(V, E, r, s, \geq)$.

Os conjuntos de arestas máximas e minímas serão denotados por $E_{\max } e$ $E_{\min }$, respectivamente.

Definição 6.1.3 (Árvore geradora). Dado um diagrama de Bratteli ordenado $\mathfrak{B}=(V, E, r, s, \geq)$. Um subconjunto de arestas $A$ é chamado de árvore geradora se para qualquer $v$ em $V$ existe um único caminho formado por arestas de $A$ de $v_{0}$ para $v$.

Proposição 6.1.4. Os conjuntos $E_{\max } e E_{\min }$ são árvores geradoras.

Prova. Dado $v$ em $V_{n}$, consideramos $v^{\prime}$ como o primeiro vértice tal que existem pelo menos duas arestas distintas $e_{1}$ e $e_{2}$ tais que $r\left(e_{1}\right)=r\left(e_{2}\right)=v^{\prime}$. Só uma destas arestas pode ser máxima, já que elas tem extremidade no mesmo vértice. Portanto, existe somente um caminho em $E_{\max }$ ligando $v$ a $v_{0}$. A situação é análoga para $E_{\text {min }}$. 
Dado um diagrama de Bratteli $\mathfrak{B}=(V, E, r, s)$ e um subconjunto $F$ de $E$, denotaremos o conjunto dos caminhos infinitos de $F$ por $X_{F}$.

Dado um diagrama de Bratteli ordenado $\mathfrak{B}=(V, E, r, s, \geq)$, definimos os conjuntos $X_{E_{\max }}$ e $X_{E_{\min }}$ como os conjuntos dos caminhos infinitos formados pelas arestas de $E_{\max }$ e $E_{\min }$, respectivamente. Definiremos uma função $\phi_{\mathfrak{B}}$ associada a $\mathfrak{B}$, que será um homeomorfismo entre $X_{\mathfrak{B}} \backslash X_{E_{\max }}$ e $X_{\mathfrak{B}} \backslash X_{E_{\min }}$.

Dado $\mathbf{x}=\left(x_{i}\right)_{i \geq 1}$ em $X_{\mathfrak{B}} \backslash X_{E_{\max }}$, existe $n$ tal que $x_{n}$ é a primeira aresta que não está em $E_{\max }$, então existe aresta $y_{n}$, tal que $r\left(x_{n}\right)=r\left(y_{n}\right)$ e $y_{n}$ é o elemento seguinte a $x_{n}$, que está unicamente determinado. Como $E_{\min }$ é árvore geradora existe um único caminho em $E_{\min }$ ligando $v_{0}$ a $s\left(y_{n}\right)$, denotemos tal caminho por $\left(y_{1}, \ldots, y_{n-1}\right)$. Assim, definimos a função

$$
\begin{aligned}
\phi_{\mathfrak{B}}: & X_{\mathfrak{B}} \backslash X_{E_{\max }} \rightarrow X_{\mathfrak{B}} \backslash X_{E_{\min }} \\
& \phi_{\mathfrak{B}}(\mathbf{x})=\left(y_{1}, \ldots, y_{n}, x_{n+1}, \ldots\right) .
\end{aligned}
$$

Proposição 6.1.5. Dado um diagrama de Bratteli ordenado $\mathfrak{B}=(V, E, r, s, \geq)$, a função $\phi_{\mathfrak{B}}$ é um homeomorfismo.

Prova. Dado y em $X_{\mathfrak{B}} \backslash X_{E_{\min }}$, tal que $y_{n}$ é a primeira entrada que não está em $E_{\text {min }}$. Existe aresta $x_{n}$, tal que $r\left(y_{n}\right)=r\left(x_{n}\right)$ e $x_{n}$ é o elemento seguinte a $y_{n}$. Como $E_{\max }$ é uma árvore geradora, existe um único caminho em $E_{\max }$ ligando $v_{0}$ a $s\left(x_{n}\right)$, denotemos tal caminho por $\left(x_{1}, \ldots, x_{n-1}\right)$. Definimos a função

$$
\begin{aligned}
\psi_{\mathfrak{B}}: & X_{\mathfrak{B}} \backslash X_{E_{\min }} \rightarrow X_{\mathfrak{B}} \backslash X_{E_{\max }} \\
& \psi_{\mathfrak{B}}(\mathbf{y})=\left(x_{1}, \ldots, x_{n}, y_{n+1}, \ldots\right) .
\end{aligned}
$$

Mostraremos que $\psi_{\mathfrak{B}}$ á a inversa de $\phi_{\mathfrak{B}}$.

Dado $\mathbf{x}$ em $X_{\mathfrak{B}} \backslash X_{\max }$, seja $x_{n}$ a primeira entrada que não está em $E_{\max } \mathrm{e}$ $y_{n}$ a aresta seguinte a $x_{n}$. Seja $\left(y_{1}, \ldots, y_{n-1}\right)$ o único caminho em $E_{\text {min }}$ ligando $v_{0}$ a $s\left(x_{n}\right)$, temos que:

$$
\psi_{\mathfrak{B}} \circ \phi_{\mathfrak{B}}(\mathbf{x})=\psi_{\mathfrak{B}}\left(\left(y_{1}, \ldots, y_{n-1}, y_{n}, x_{n+1}, \ldots\right)\right)=\left(x_{1}, \ldots, x_{n}, x_{n+1}, \ldots\right)=\mathbf{x}
$$

Agora, dado y em $X_{\mathfrak{B}} \backslash X_{\min }$, seja $y_{n}$ a primeira entrada que não está em $E_{\text {min }} \mathrm{e}$ $x_{n}$ a aresta seguinte a $y_{n}$. Seja $\left(x_{1}, \ldots, x_{n-1}\right)$ o único caminho em $E_{\max }$ ligando $v_{0}$ a $s\left(y_{n}\right)$, temos que:

$$
\phi_{\mathfrak{B}} \circ \psi_{\mathfrak{B}}(\mathbf{y})=\phi_{\mathfrak{B}}\left(\left(x_{1}, \ldots x_{n-1}, x_{n}, y_{n+1}, \ldots\right)\right)=\left(y_{1}, \ldots, y_{n}, y_{n+1}, \ldots\right)=\mathbf{y}
$$

Portanto, $\psi_{\mathfrak{B}}$ é a inversa de $\phi_{\mathfrak{B}}$. Logo $\phi_{\mathfrak{B}}$ é bijeção. 
Falta mostrar que $\phi_{\mathfrak{B}}$ e $\psi_{\mathfrak{B}}$ são contínuas. Provaremos a continuidade de $\phi_{\mathfrak{B}}$. A continuidade de $\psi_{\mathfrak{B}}$ se prova de forma análoga. Dados x em $X_{\mathfrak{B}} \backslash X_{E_{\max }}$ e $\epsilon>0$, existem $\ell>0$ tal que $x_{\ell}$ é a primeira entrada que não é máxima $n$ em $\mathbb{N}$, tal que $2^{-n}=\epsilon$. Considere $\delta=\epsilon$, mostraremos que se $\mathbf{z}$ está em $B(\mathbf{x}, \delta)$, então $\phi_{\mathfrak{B}}(\mathbf{z})$ está em $B\left(\phi_{\mathfrak{B}}(\mathbf{x}), \epsilon\right)$.

Digamos que $\phi_{\mathfrak{B}}(\mathbf{x})=\left(y_{1}, \ldots, y_{\ell}, x_{l+1}, \ldots\right)$. Temos dois casos. O primeiro é quando $\ell \leq k$. Neste caso, temos que $x_{i}=z_{i}$ e que $x_{i}$ e $z_{i}$ estão em $E_{\max }$ para todo $0 \leq i \leq \ell-1$, portanto $\left(x_{1}, \ldots, x_{\ell-1}\right)$ e $\left(z_{1}, \ldots, z_{\ell-1}\right)$ serão levados por $\varphi_{\mathfrak{B}}$ no mesmo caminho mínimo $\left(y_{1}, \ldots, y_{\ell-1}\right)$. Além disso, $x_{\ell}=z_{l}$, assim $y_{\ell}$ também é o elemento seguinte para $z_{\ell}$. Então, temos que

$$
\phi_{\mathfrak{B}}(\mathbf{z})=\left(y_{1}, \ldots, y_{\ell}, z_{\ell+1}, \ldots\right)=\left(y_{1}, \ldots, y_{\ell}, x_{\ell+1} \ldots, x_{k}, z_{k+1}, \ldots\right) .
$$

Assim, $\left(\phi_{\mathfrak{B}}(\mathbf{x})\right)_{i}=\left(\phi_{\mathfrak{B}}(\mathbf{z})\right)_{i}$ para todo $0 \leq i \leq k, \log 0 \phi_{\mathfrak{B}}(\mathbf{z}) \in B\left(\phi_{\mathfrak{B}}(\mathbf{x}), 2^{-k}\right) \subset$ $B\left(\phi_{\mathfrak{B}}(\mathbf{x}), \epsilon\right)$.

Agora considere que $\ell>k$. Temos que $x_{i}=z_{i}$ e que $x_{i}$ e $z_{i}$ estão em $E_{\max }$ para todo $1 \leq i \leq \ell-1$, portanto $\left(x_{1}, \ldots, x_{\ell-1}\right)$ e $\left(z_{1}, \ldots, z_{\ell-1}\right)$ são levados por $\phi_{\mathfrak{B}}$ no mesmo caminho mínimo $\left(y_{1}, \ldots, y_{\ell-1}\right)$. Assim, $\left(\phi_{\mathfrak{B}}(\mathbf{x})\right)_{i}=\left(\varphi_{\mathfrak{B}}\right)(\mathbf{z})_{i}$ para todo $1 \leq i \leq \ell-1$. Então $\phi_{\mathfrak{B}}(\mathbf{z}) \in B\left(\phi_{\mathfrak{B}}(\mathbf{x}), 2^{-(\ell-1)}\right) \subset B\left(\phi_{\mathfrak{B}}(\mathbf{x}), \epsilon\right)$.

Concluímos, assim, a demonstração da proposição.

Definição 6.1.6 (Diagrama de Bratteli próprio). Dado um diagrama de Bratteli ordenado $\mathfrak{B}=(V, E, r, s, \geq)$, dizemos que $\mathfrak{B}$ é próprio se $E_{\max }$ e $E_{\min }$ possuem, cada um, exatamente um caminho infinito. Também dizemos que $\geq$ é uma ordem própria em $(V, E, r, s)$.

Em um diagrama de Bratteli ordenado próprio, denotamos por $\mathbf{x}_{\max } \mathrm{e}$ $\mathbf{x}_{\text {min }}$ os únicos caminhos infinitos de $E_{\max }$ e $E_{\min }$, respectivamente. Neste caso, podemos estender o domínio da função $\phi_{\mathfrak{B}}$ para $X_{\mathfrak{B}}$. Definimos a extensão de $\phi_{\mathfrak{B}}$ por

$$
\begin{aligned}
\varphi_{\mathfrak{B}} & : X_{\mathfrak{B}} \rightarrow X_{\mathfrak{B}} \\
& \left.\varphi_{\mathfrak{B}}\right|_{X_{\mathfrak{B}} \backslash X_{E_{\max }}}=\phi_{\mathfrak{B}} \mathrm{e} \\
& \varphi_{\mathfrak{B}}\left(\mathbf{x}_{\max }\right)=\mathbf{x}_{\text {min }}
\end{aligned}
$$

Teorema 6.1.7. Dado um diagrama de Bratteli ordenado próprio $\mathfrak{B}=$ $(V, E, r, s, \geq)$, a função $\varphi_{\mathfrak{B}}$ é um homeomorfismo.

Prova. Por hipótese, $\mathfrak{B}$ é próprio, logo $X_{E_{\max }}=\left\{\mathbf{x}_{\max }\right\}$, assim

$$
X_{\mathfrak{B}} \backslash X_{E_{\max }}=X_{\mathfrak{B}} \backslash\left\{\mathbf{x}_{\max }\right\} .
$$


Além disso, $\varphi_{\mathfrak{B}}\left(\mathbf{x}_{\max }\right)=\mathbf{x}_{\min }$, então

$$
X_{\mathfrak{B}} \backslash X_{E_{\min }}=X_{\mathfrak{B}} \backslash\left\{\varphi_{\mathfrak{B}}\left(\mathbf{x}_{\max }\right)\right\}
$$

Pela Proposição 6.1.5, $\left.\varphi_{\mathfrak{B}}\right|_{X_{E_{\max }} \backslash\left\{x_{\max }\right\}}$ é um homeomorfismo. Então é suficiente mostrar que $\varphi_{\mathfrak{B}}$ é contínua em $\mathbf{x}_{\max }$. Se $\mathbf{x}_{\max }$ é ponto isolado não há o que fazer. Agora, considere que $\mathbf{x}_{\max }$ não é um ponto isolado. Definimos a sequência $\left\{\mathbf{x}_{n}\right\}_{n \geq 1}$, onde para cada $n \geq 1, \mathbf{x}_{n}=\left(x_{n, i}\right)_{n \geq 1}$ está em $X_{\mathfrak{B}}$ e $x_{n, i}$ está em $E_{\max }$ para todo $1 \leq i \leq n$. Temos que $\lim _{n \rightarrow \infty} \mathbf{x}_{n}=\mathbf{x}$. Para cada $n \geq 1, f\left(\mathbf{x}_{n}\right)=\mathbf{y}_{n}$, onde $y_{i, n}$ está em $E_{\min }$ para todo $1 \leq i \leq n$, assim $\lim _{n \rightarrow \infty} f\left(\mathbf{x}_{n}\right)=\mathbf{x}_{\text {min }}=f\left(\mathbf{x}_{\max }\right)$. Concluímos que $\varphi_{\mathfrak{B}}$ é contínua em $\mathbf{x}_{\max } \mathrm{e}$ portanto é um homeomorfismo.

Chamaremos a função $\varphi_{\mathfrak{B}}: X_{\mathfrak{B}} \rightarrow X_{\mathfrak{B}}$ de aplicação de Bratteli-Vershik associada ao diagrama de Bratteli $\mathfrak{B}$.

Teorema 6.1.8. Dado um diagrama de Bratteli $\mathfrak{B}=(V, E, r, s)$. Se $\mathfrak{B}$ possui todas as arestas conectadas, então $\mathfrak{B}$ possui uma ordem própria.

Prova. Para cada $n \geq 1$, denotamos os vértices em $V_{n}$ por $v_{i}^{n}$, onde $1 \leq i \leq \# V_{n}:=m(n)$. Dado $v_{j}^{n}$ em $V_{n}$, como $\mathfrak{B}$ possui todas as arestas conectadas, temos que $r^{-1}(v)=V_{n-1}$. Definimos, então $e_{n, i, j}$ a aresta em $E_{n}$ que liga $v_{i}^{n-1}$ em $V_{n-1}$ a $v_{j}^{n}$ em $V_{n}$. Escolhemos como aresta mínima $e_{(n, 1, j)}$ e como aresta máxima $e_{(n, m(n-1), j)}$. Temos que $r\left(e_{(n-1,1,1)}\right)=s\left(e_{(n, 1,1)}\right)$ e $s\left(e_{(n-1, m(n-2), m(n-1))}\right)=r\left(e_{(n, m(n-1), m(n))}\right)$ para todo $n$, assim definimos:

$$
\begin{gathered}
\mathbf{x}_{\min }=\left(e_{(1,1,1)}, e_{(2,1,1)} \ldots e_{(n, 1,1)}, \ldots\right) \mathrm{e} \\
\mathbf{x}_{\max }=\left(e_{(1, m(0), m(1))}, e_{(1, m(1), m(2))}, \ldots, e_{(1, m(n-1), m(n))}, \ldots\right) .
\end{gathered}
$$

Mostraremos que $\mathbf{x}_{\min }$ é o único caminho mínimo. Suponha que existe outro caminho mínimo $\mathbf{y}$ diferente de $\mathbf{x}_{m i n}$, então existe para algum $n$ vértice $v_{i}^{n}$, onde $i$ é diferente de 1 e $v_{i}^{n}$ é a imagem por $r$ de alguma aresta mínima em $E_{n}$, mas, por definição, nenhuma aresta em $s\left(v_{i}^{n}\right)$ é mínima. Portanto y não pode ser um caminho mínimo. Analogamente mostramos que $\mathbf{x}_{\max }$ é o único caminho máximo.

Então construímos uma ordem própria para o diagrama $\mathfrak{B}$ e portanto terminamos a demonstração.

Exemplo 6.1.9. O diagrama de Bratteli da Figura 6.1 é próprio. 


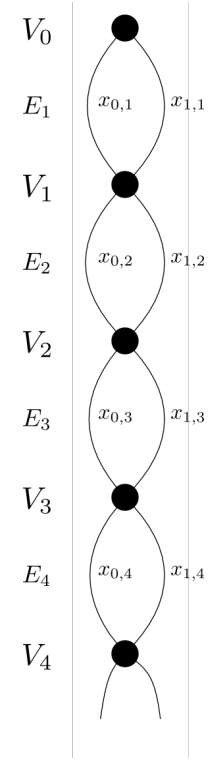

Figura 6.1: Diagrama de Bratteli próprio.

Definimos que $x_{\min , n}=x_{0,1}$ e que $x_{\max , n}=x_{1,1}$. Assim, só há um caminho infinito formado somente por arestas máximas e somente um formado por arestas mínimas

Exemplo 6.1.10. Os diagramas $\mathfrak{T}$ e $\mathfrak{Q}$ apresentados, respectivamente, nas figuras 6.2 e 6.3 não são próprios, já que cada vértice só recebe uma aresta. Portanto, todo aresta é máxima e também minima.

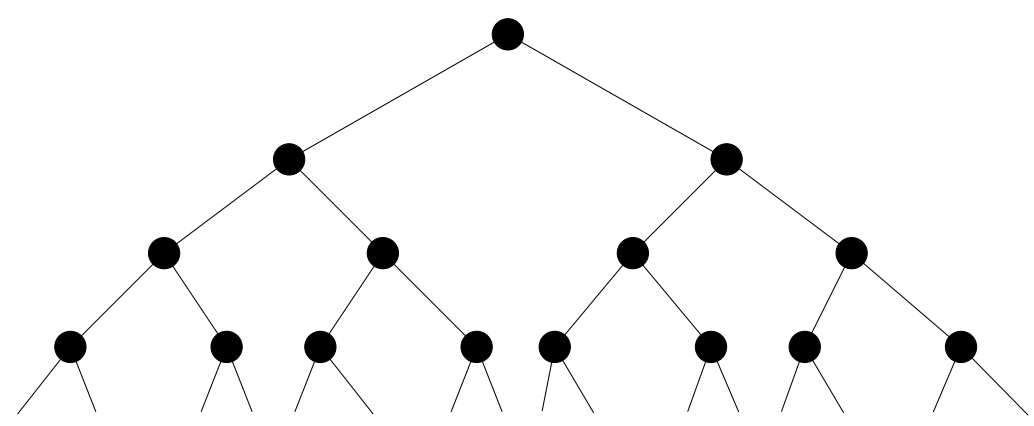

Figura 6.2: Diagrama de Bratteli $\mathfrak{T}$ 


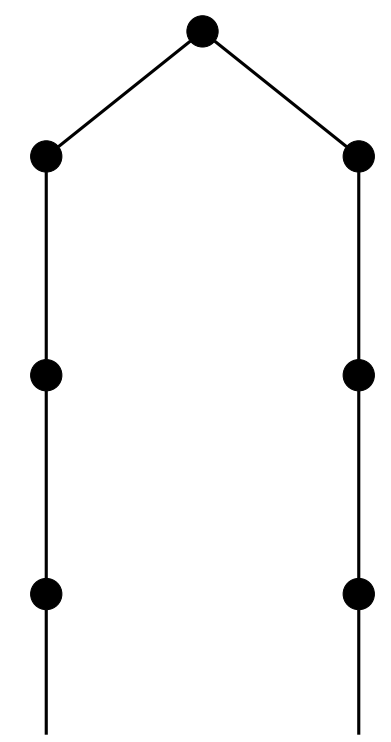

Figura 6.3: Diagrama de Bratteli $\mathfrak{Q}$

\section{2}

As relações $R_{\varphi_{\mathfrak{B}}}$ e $R_{\mathfrak{B}}$

Seja $\mathfrak{B}$ um diagrama de Bratteli. O objetivo desta seção é estudar a relação entre $R_{\varphi_{\mathfrak{B}}}$ e $R_{\mathfrak{B}}$. Lembre que dados x e y em $X_{\mathfrak{B}}$ definimos:

$-\mathbf{x} \sim_{R_{\mathfrak{B}}} \mathbf{y}$ se e só se $\mathbf{x}$ e $\mathbf{y}$ diferem em um número finito de entradas. Em particular, existe $n \geq 1$ tal que $x_{i}=y_{i}$ para todo $i>n$. Representamos a classe de equivalência de $\mathbf{x}$ por $\langle\mathbf{x}\rangle_{R_{\mathfrak{B}}}$.

$-\mathbf{x} \sim_{R_{\varphi_{\mathfrak{B}}}} \mathbf{y}$ se e só se existe inteiro $k$ tal que $\varphi_{\mathfrak{B}}^{k}(\mathbf{x})=\mathbf{y}$. Representamos a classe de equivalência de $\mathbf{x}$ por $R_{\varphi_{\mathfrak{B}}}$.

Para cada $n \geq 0$, consideramos a sub-relação de equivalência $R_{\mathfrak{B}, n}$ de $R_{\mathfrak{B}}$ em $X_{\mathfrak{B}}$, tal que $\mathbf{x} \sim_{R_{\mathfrak{B}, n}} \mathbf{y}$ se e só se $x_{i}=y_{i}$ para todo $i>n$. Denotaremos a classe de equivalência de $\mathbf{x}$ por $\langle\mathbf{x}\rangle_{R_{\mathfrak{B}, n}}$. Note que para todo $\mathbf{x} \in X_{\mathfrak{B}}$, $\langle\mathbf{x}\rangle_{R_{\mathfrak{B}, n}} \subset\langle\mathbf{x}\rangle_{R_{\mathfrak{B}}}$.

Agora iremos analisar o comportamento da relação orbital $R_{\varphi_{\mathfrak{B}}}$ quando $\mathfrak{B}$ é um diagrama de Bratteli ordenado próprio.

Teorema 6.2.1. Dado um diagrama de Bratteli ordenado $\mathfrak{B}=(V, E, r, s, \geq)$. As seguintes afirmações são verdadeiras:

1. Se $\mathbf{x} \notin X_{E_{\max }}$ entãa $\mathbf{x} \sim_{R_{\mathfrak{B}}} \varphi_{\mathfrak{B}}(\mathbf{x})$.

2. $R_{\mathfrak{B}}$ é a menor relação de equivalência na qual $\mathbf{x} \sim_{R_{\mathfrak{B}}} \varphi_{\mathfrak{B}}(\mathbf{x})$ para todo $\mathbf{x}$ em $X_{\mathfrak{B}} \backslash X_{E_{\max }}$. 
3. Se $\mathfrak{B}$ é próprio então $\langle\mathbf{x}\rangle_{R_{\mathfrak{B}}} \subset\langle\mathbf{x}\rangle_{R_{\varphi_{\mathfrak{B}}}}$ para todo $\mathbf{x}$ em $X_{\mathfrak{B}}$ e $\mathbf{x}_{\max } \sim_{R_{\varphi_{\mathfrak{B}}}}$ $\mathbf{x}_{\min }$.

4. Se $\mathfrak{B}$ é próprio, então $R_{\varphi_{\mathfrak{B}}}$ é a menor relação de equivalência tal que para todo $\mathbf{x}$ em $X_{\mathfrak{B}},\langle\mathbf{x}\rangle_{R_{\mathfrak{B}}} \subset\langle\mathbf{x}\rangle_{R_{\varphi_{\mathfrak{B}}}}$ e $\mathbf{x}_{\max } \sim_{R_{\varphi_{\mathfrak{B}}}} \mathbf{x}_{\min }$.

Prova. Precisaremos do seguinte lema:

Lema 6.2.2. Considere um diagrama de Bratteli ordenado $\mathfrak{B}=(V, E, r, s, \geq)$, $\mathbf{x}$, y em $X_{\mathfrak{B}}$ e $n \geq 1$. Se $\mathbf{x} \sim_{R_{\mathfrak{B}, n}} \mathbf{y}$ e $x_{n}<y_{n}$ então existe $k>0$ tal que $\varphi_{\mathfrak{B}}^{i}(\mathbf{x})$ não está em $X_{E_{\max }}$ para todo $0 \leq i<k$ e $\varphi_{\mathfrak{B}}^{k}(\mathbf{x})=\mathbf{y}$.

Prova. A prova será por indução em $n$. Há uma pequena diferença para o caso $n=1$ e portanto ele será feito separadamente.

Dados $\mathbf{x}$ e $\mathbf{y}$ em $X_{\mathfrak{B}}$ tais que $\mathbf{x} \sim_{R_{\mathfrak{B}, 1}} \mathbf{y}$ e $x_{1}<y_{1}$. Se $m$ é a quantidade de arestas entre $x_{1}$ e $y_{1}$, segue, pela definição de $\varphi_{\mathfrak{B}}$, que $\varphi^{m+1}(\mathbf{x})=\mathbf{y}$.

O caso $n=2$ será importante para entender o caso geral e portanto ele também será feito.

- Caso $\mathrm{n}=2$ : por hipótese $\mathbf{x} \sim_{R_{\mathfrak{B}, 2}} \mathbf{y}$ e $x_{2}<y_{2}$. Temos que $r\left(x_{2}\right)=r\left(y_{2}\right)=v$, Listamos na ordem $\geq$ todas as arestas entre $x_{2}$ e $y_{2}$ levadas por $r$ em $v$ :

$$
x_{2}=e_{2,1}<e_{2,2}<\cdots<e_{2, m}=y_{2},
$$

onde $m \geq 2$.

Agora ordenamos as arestas em $E_{1}$ :

$$
e_{1,1}<e_{1,2}<\cdots<e_{1, r}
$$

Então, temos dois casos. Se $x_{1}=e_{1, r}$ :

$$
\varphi_{\mathfrak{B}}^{1}(\mathbf{x})=\left(e_{1,1}, e_{2,2}, x_{3}, \ldots\right)
$$

Agora, se $x_{1}=e_{1, j}$, onde $j \neq r$, temos:

$$
\varphi_{\mathfrak{B}}^{r-j}(\mathbf{x})=\left(e_{1, r}, e_{2,1}=x_{2}, x_{3}, \ldots\right)
$$

Definimos $j-r=\ell$. Então

$$
\varphi_{\mathfrak{B}}^{\ell+1}(\mathbf{x})=\left(e_{1,1}, e_{2,2}, x_{3}, \ldots\right)
$$

Agora, temos que:

$$
\varphi_{\mathfrak{B}}^{\ell+1+r}(\mathbf{x})=\left(e_{1,1}, e_{2,3}, x_{3}, \ldots\right)
$$


Seguindo indutivamente, obtemos:

$$
\varphi_{\mathfrak{B}}^{\ell+1+r(m-2)}(\mathbf{x})=\left(e_{1,1}, e_{2, m}=y_{2}, x_{3}, \ldots\right)
$$

para $m \geq 2$. Temos que $y_{1}=e_{1, j^{\prime}}$, então

$$
\varphi_{\mathfrak{B}}^{\ell+1+r(m-2)+j^{\prime}-1}(\mathbf{x})=\left(y_{1}, y_{2}, x_{3}, \ldots\right)
$$

Como, por hipótese $\mathbf{x} \sim \mathbf{y}_{R_{\mathfrak{B}, 2}}$ temos que $x_{i}=y_{i}$ para todo $i>2$, então

$$
\varphi_{\mathfrak{B}}^{\ell+1+r(m-2)+j^{\prime}-1}(\mathbf{x})=\left(y_{1}, y_{2}, x_{3}, \ldots\right)=\mathbf{y}
$$

- Suponha que $\mathbf{x} \sim_{R_{\mathfrak{B}, i}} \mathbf{y}$ para $1 \leq i \leq n-1$ e que existe $k$ tal que $\varphi_{\mathfrak{B}}^{j}(\mathbf{x})$ não está em $X_{E_{\max }}$ para todo $0 \leq j<k$ e $\varphi_{\mathfrak{B}}^{k}(\mathbf{x})=\mathbf{y}$.

- Dados $\mathbf{x}$ e $\mathbf{y}$ em $X_{\mathfrak{B}}$, tais que $\mathbf{x} \sim_{R_{E, n}} \mathbf{y}$ e $x_{n}<y_{n}$. Listaremos em ordem todas as arestas levadas por $r$ em $r\left(x_{n}\right)=r\left(y_{n}\right)$ :

$$
x_{n}=e_{n, 1}<e_{n, 2}<\cdots<e_{n, m}=y_{n} .
$$

Como $E_{\max }$ e $E_{\min }$ são árvores geradoras, podemos definir para cada $i \geq 1$, os caminhos $p_{i}$ e $q_{i}$ como os únicos caminhos mínimo e máximo, respectivamente, que ligam $v_{0}$ a $s\left(e_{i}\right)$. Consideremos a lista de pontos:

$$
\begin{aligned}
& \mathbf{x}=\left(x_{1}, x_{2}, \ldots, x_{n-1} \quad, \quad x_{n}=e_{1} \quad, \quad x_{n+1}, \ldots\right) \\
& \mathbf{z}_{1}=\left(q_{1} \quad, \quad e_{1} \quad, x_{n+1}, \ldots\right) \\
& \mathbf{w}_{2}=\left(p_{2} \quad, \quad e_{2} \quad, x_{n+1}, \ldots\right) \\
& \mathbf{z}_{2}=\left(\begin{array}{lll}
q_{2} & \left.e_{2} \quad, x_{n+1}, \ldots\right)
\end{array}\right) \\
& \mathbf{w}_{3}=\left(\quad p_{3} \quad, \quad e_{3} \quad, x_{n+1}, \ldots\right) \\
& \mathbf{z}_{3}=\left(q_{3} \quad, \quad e_{3} \quad, x_{n+1}, \ldots\right) \\
& \mathbf{z}_{m-1}=\left(q_{m-1} \quad, \quad e_{m-1} \quad, x_{n+1}, \ldots\right) \\
& \mathbf{w}_{m}=\left(p_{m} \quad, \quad e_{m} \quad, x_{n+1}, \ldots\right) \\
& \mathbf{y}=\left(y_{1}, y_{2}, \ldots, y_{n-1} \quad, \quad y_{n}=e_{m} \quad, \quad x_{n+1}, \ldots\right) \text {. }
\end{aligned}
$$

Denotaremos o segundo ponto da lista por $\mathbf{z}$, temos que $\mathbf{x} \sim_{R_{E, n-1}} \mathbf{y}$. Se $q_{1}=x_{1}, \ldots, x_{n-1}$ então $\mathbf{x}$ e $\mathbf{z}$ são iguais. Suponha que são diferentes então existe $t$ tal que $x_{t}$ é a primeira aresta que não está em $E_{\max } \mathrm{e}$ $1 \leq t \leq n-1$. Usando a hipótese de indução temos que existe $k_{1} \geq 0$, tal que $\varphi_{\mathfrak{B}}^{k_{1}}(\mathbf{x})=\mathbf{z}$. Temos, pela definição de $\varphi_{\mathfrak{B}}$, que $\varphi_{\mathfrak{B}}$ aplicada ao segundo ponto é o terceiro ponto. 
Usando os argumentos acima alternadamente, obtemos que cada ponto da lista é alguma potência inteira de $\varphi_{\mathfrak{B}}$ aplicada ao ponto anterior. Assim, existe $K>0$, tal que $\varphi_{\mathfrak{B}}^{K}(\mathbf{x})=\mathbf{y}$.

Finalizamos, então, a demonstração do lema.

Agora voltaremos à demonstração do Teorema 6.2.1.

1. Se $\mathbf{x} \notin X_{E_{\max }}$, a função $\varphi_{\mathfrak{B}}$ altera apenas um número finito de entradas e após a primeira entrada que é igual a $\mathbf{x}_{\min }$ não há alterações. Então existe $n>0$ tal que $x_{i}=\left(\varphi_{\mathfrak{B}}(\mathbf{x})\right)_{i}$ para todo $i>n$. Portanto $\mathbf{x} \sim_{R_{\mathfrak{B}}} \varphi_{\mathfrak{B}}(\mathbf{x})$.

2. Suponha que existe relação de equivalência $R$ tal que $\langle\mathbf{x}\rangle_{R} \subset\langle\mathbf{x}\rangle_{R_{\mathfrak{B}}}$ para todo $\mathbf{x}$ em $X_{\mathfrak{B}}$ e $\mathbf{x} \sim_{R} \varphi_{\mathfrak{B}}(\mathbf{x})$ para todo $\mathbf{x}$ em $X_{\mathfrak{B}} \backslash X_{E_{\max }}$.

Sejam $\mathbf{x}$ e $\mathbf{y}$ distintos em $X_{\mathfrak{B}} \backslash E_{\max }$ tais que $\mathbf{x} \sim_{R_{\mathfrak{B}}} \mathbf{y}$. Pelo Lema 6.2.2, temos que existe $m>0$ tal que $\varphi_{\mathfrak{B}}^{m}(\mathbf{x})=\mathbf{y}$ e nenhum dos pontos $\mathbf{x}, \varphi_{\mathfrak{B}}(\mathbf{x}), \ldots, \varphi_{\mathfrak{B}}^{m-1}(\mathbf{x})$ está em $X_{E_{\max }}$. Então, por hipótese, para cada $0 \leq i<m, \varphi_{\mathfrak{B}}^{i}(\mathbf{x}) \sim_{R} \varphi^{i+1}(\mathbf{x})$. Por transitividade, $\mathbf{x} \sim_{R} \mathbf{y}$. Assim. $\langle\mathbf{x}\rangle_{R_{\mathfrak{B}}} \subset\langle\mathbf{x}\rangle_{R}$ e, portanto, $R=R_{\mathfrak{B}}$.

3. Por hipótese, $\mathfrak{B}$ é próprio. Pela definição de $\varphi_{\mathfrak{B}}$ temos que, $\varphi_{\mathfrak{B}}\left(\mathbf{x}_{\max }\right)=$ $\mathbf{x}_{\min }$, então $\mathbf{x}_{\max } \sim_{R_{\varphi_{\mathfrak{B}}}} \mathbf{x}_{\min }$. Além disso, temos que $\mathbf{x} \sim_{\varphi_{\mathfrak{B}}} \varphi_{\mathfrak{B}}(\mathbf{x})$ para todo $\mathbf{x}$ em $X_{\mathfrak{B}} \backslash X_{E_{\max }}$. Pelo item anterior, concluímos que $\langle\mathbf{x}\rangle_{R_{\mathfrak{B}}} \subset$ $\langle\mathbf{x}\rangle_{R_{\varphi_{\mathfrak{B}}}}$.

4. Seja relação de equivalência $R$, tal que $\langle\mathbf{x}\rangle_{R_{\mathfrak{B}}} \subset\langle\mathbf{x}\rangle_{R} \subset\langle\mathbf{x}\rangle_{R_{\varphi_{\mathfrak{B}}}}$ e que $\mathbf{x}_{\max } \sim_{R} \mathbf{x}_{\text {min }}$. Então $\mathbf{x} \sim_{R} \varphi_{\mathfrak{B}}(\mathbf{x})$ para todo $\mathbf{x} \in X_{\mathfrak{B}}$. Pela transitividade da relação de equivalência $R$, temos que $\mathbf{x} \sim_{R} \varphi_{\mathfrak{B}}^{m}(\mathbf{x})$ para todo $\mathbf{x} \in X_{\mathfrak{B}}$ e $m \geq 0$. Como $R$ é simétrica, também temos que $\varphi_{\mathfrak{B}}^{m}(\mathbf{x}) \sim_{R} \mathbf{x}$ para todo $\mathbf{x} \in X_{\mathfrak{B}}$ e $m \geq 0$. Agora, considere $m<0$, temos que $\varphi_{\mathfrak{B}}^{-m}\left(\varphi_{\mathfrak{B}}^{m}(\mathbf{x})\right) \sim_{R} \varphi_{\mathfrak{B}}^{m}(x)$. Portanto, $\mathbf{x} \sim_{R} \varphi_{\mathfrak{B}}^{m}(\mathbf{x})$ para todo $\mathbf{x} \in X_{\mathfrak{B}} \mathrm{e}$ todo $m<0$. Concluímos, então que $\langle\mathbf{x}\rangle_{R_{\varphi_{\mathfrak{B}}}} \subset\langle\mathbf{x}\rangle_{R}$, logo $R=R_{\varphi_{\mathfrak{B}}}$.

Concluímos, então, a demonstração do teorema.

Agora iremos apresentar um resultado sobre a minimalidade de $R_{\mathfrak{B}}$.

Teorema 6.2.3. Dado um diagrama de Bratteli próprio $\mathfrak{B}=(V, E, r, s, \geq)$. As seguintes afirmações são equivalentes:

1. a relação de equivalência $R_{\varphi_{\mathfrak{B}}}$ é minimal;

2. a relação de equivalência $R_{\mathfrak{B}}$ é minimal; 
3. o diagrama $\mathfrak{B}$ é simples.

Prova. Pelo Teorema 5.3.5, temos que (2) e (3) são equivalentes e pelo Teorema 6.2.1 temos que $(2) \Rightarrow(1)$, então falta mostrar que $(1) \Rightarrow(2)$. Suponha que $R_{\varphi_{\mathfrak{B}}}$ é minimal. Pelo Teorema 6.2.1, temos que para todo $\mathbf{x} \in X_{\mathfrak{B}} \backslash\left\{\mathbf{x}_{\max }\right\}$, $\langle\mathbf{x}\rangle_{R_{\mathfrak{B}}}=\langle\mathbf{x}\rangle_{\varphi_{\mathfrak{B}}}$. Como $R_{\varphi_{\mathfrak{B}}}$ é minimal, para todo $\mathbf{x} \in X_{\mathfrak{B}} \backslash\left\{\mathbf{x}_{\max }\right\}$ a classe de equivalência $\langle\mathbf{x}\rangle_{R_{\mathfrak{B}}}$. Portanto, só falta mostrar que $\left\langle\mathbf{x}_{\max }\right\rangle_{R_{\mathfrak{B}}}$ é densa. Sejam $\mathbf{z} \in X_{\mathfrak{B}}$ e $r<0$. Existe $n>0$ tal que $2^{-n}<r$. Definimos o elemento

$$
\mathbf{y}=\left(z_{1}, \ldots, z_{n} x_{\max _{n+1}}, x_{\max _{n+2}}, \ldots\right)
$$

Por definição,

$$
\mathbf{y} \in B(z, r) \bigcap\left\langle\mathbf{x}_{\max }\right\rangle_{R_{\mathfrak{B}}} .
$$

Concluímos, então que $\left\langle\mathbf{x}_{\max }\right\rangle_{R_{\mathfrak{B}}}$ é densa e, portanto, finalizamos a demonstração do teorema.

No Capítulo 2 definimos a soma no espaço $\{0,1\}^{\mathbb{N}}$. Considere, agora, o espaço de sequências infinitas $\mathbf{x}=\left(x_{i}\right)_{i \geq 1}$, onde para cada $i \geq 1$, existe um número natural $a_{n}>1$, tal que $x_{i} \in\left\{0,1, \ldots, a_{n}-1\right\}$. Podemos representar este espaço por

$$
X=\prod_{n=1}^{\infty}\left\{0,1, \ldots, a_{n}-1\right\} .
$$

Dados x e y em $X$, definimos $z_{1}=\left(x_{1}+y_{1}\right) \bmod a_{1}$,

$$
w_{1}= \begin{cases}1, & \text { se } x_{1}+y_{1} \geq a_{1} \\ 0, & \text { se } x_{1}+y_{1}<a_{1}\end{cases}
$$

Para $i \geq 1$, definimos

$$
w_{i+1}= \begin{cases}1, & \text { se } x_{i}+y_{i} \geq a_{i} \\ 0, & \text { se } x_{i}+y_{i}<a_{i}\end{cases}
$$

Definimos, também, $z_{i+1}=\left(w_{i}+x_{i+1}+y_{i+1}\right) \bmod a_{i+1}$. Por fim,

$$
\mathbf{x}+\mathbf{y}:=\mathbf{z}=\left(z_{i}\right)_{i \geq 1}
$$

Considere o diagrama de Bratteli tal que $V_{n}=\left\{v_{n}\right\}$ para todo $n \geq 0$ e que para cada $n \geq 1, E_{n}=\left\{0,1, \ldots, a_{n}-1\right\}$. Como para cada $n \geq 0$, o conjunto $V_{n}$ é unitário, as funções $r$ e $s$ estão unicamente determinadas. Ademais, se denotamos um elemento de $E_{n}$ por $e_{i, n}$, onde $1 \leq i \leq a_{n}-1$, temos que $r\left(e_{i, n}\right)=r\left(e_{j, n}\right)$ para todo $1 \leq i, j \leq a_{n}-1$. Então todas as arestas em $E_{n}$ 
podem ser comparadas. Para cada $n \geq 1$, definimos a ordem natural: $e_{i, n} \geq e_{j, n}$ se e somente se $i \geq j$. Portanto, formamos um diagrama de Bratteli ordenado e próprio $\mathfrak{B}=\left(V=\left\{V_{n}\right\}_{n \geq 0}, E=\left\{E_{n}\right\}_{n \geq 0}, r, s, \geq\right)$. Temos que

$$
X_{\mathfrak{B}}=\prod_{n=1}^{\infty}\left\{0,1, \ldots, a_{n}-1\right\}
$$

A transformação de Bratteli-Vershik associada ao diagrama $\mathfrak{B}, \varphi_{\mathfrak{B}}$, é igual, neste caso, a adição do elemento $(1000 \ldots)$ a um elemento do espaço $X_{\mathfrak{B}}$. Chamamos o sistema $\left(X_{\mathfrak{B}}, \varphi_{\mathfrak{B}}\right)$ de odômetro.

Como o diagrama $\mathfrak{B}$ possui somente um vértice em cada nível, temos que $\mathfrak{B}$ é simples. Então, pelo Teorema 6.2 .3 , a relação de equivalência $R_{\varphi_{\mathfrak{B}}}$ é minimal, e, portanto, o sistema dinâmico $\left(X_{\mathfrak{B}}, \varphi_{\mathfrak{B}}\right)$ é minimal. 


\section{7}

\section{Partições de Kakutani-Rokhlin}

O objetivo deste capítulo é mostrar que dados um conjunto de Cantor $X$, um homeomorfismo minimal $\varphi: X \rightarrow X$ e $y \in X$, existem um diagrama de Bratteli $\mathfrak{B}=(V, E, r, s, \geq)$ ordenado, simples e próprio e uma conjugação $h: X_{\mathfrak{B}} \rightarrow X$ e $h\left(\mathbf{x}_{\max }\right)=y$. Para isto precisaremos definir alguns conceitos.

\section{1}

\section{Castelos}

Nesta seção dados um conjunto de Cantor, um homeomorfismo minimal $\varphi: X \rightarrow X$ e um subconjunto clopen não vazio de $X$, produziremos uma partição do espaço $X$ associada à função $\varphi$ e ao subconjunto $Y$.

Lema 7.1.1. Sejam $X$ um espaço métrico compacto $e \varphi: X \rightarrow X$ um homeomorfismo minimal. Se $Y$ é um subconjunto fechado de $X$, tal que $\varphi(Y) \subset Y$ então ou $Y=X$ ou $Y=\emptyset$.

Prova. Suponha que $Y \neq \emptyset$. Então existe $y \operatorname{em~} Y$. Como $\varphi$ é minimal, temos que

$$
\overline{\bigcup_{i=0}^{\infty} \varphi^{i}(y)}=X .
$$

Mas, $\varphi(Y) \subset Y$, então

$$
\overline{\bigcup_{i=0}^{\infty} \varphi^{i}(y)} \subset Y .
$$

Então $X \subset Y$ e portanto $Y=X$.

Lema 7.1.2. Sejam $X$ um conjunto de Cantor, $\varphi: X \rightarrow X$ um homeomorfismo minimal e $Y$ um subconjunto de $X$ clopen e não vazio. Então:

1. Para cada y em $Y$, o conjunto

$$
\left\{n \in \mathbb{N}, n \geq 1 \mid \varphi^{n}(y) \in Y\right\}
$$

é não vazio. 
2. A função $\lambda_{Y}: Y \rightarrow \mathbb{N}$

$$
\lambda_{Y}(y)=\inf \left\{n \in \mathbb{N}, n \geq 1 \mid \varphi^{n}(y) \in Y\right\}
$$

está bem definida e para cada y em $Y$ é contínua.

3. O conjunto $\lambda_{Y}(Y)$ é finito.

Prova. Veremos que as afirmações são verdadeiras.

1. Dado $y$ em $Y$, como $Y$ é aberto existe vizinhança $V_{y}$ de $Y$, tal que $V_{y} \subset Y$. Como $\varphi$ é minimal, $\mathcal{O}_{\varphi}(y) \cap V_{y} \neq \emptyset$. Portanto o conjunto

$$
\left\{n \in \mathbb{N} n \geq 1 \mid \varphi^{n}(y) \in Y\right\}
$$

é não vazio.

2. Pelo item anterior, temos que a função está bem definida. Dado y em $Y$, considere sequência $\left\{x_{k}\right\}_{k \in \mathbb{N}}$ de elementos de $Y$ tal que

$$
\lim _{k \rightarrow \infty} x_{k} \rightarrow y
$$

Definimos $\lambda_{Y}(y)=n$. Como $\varphi$ é homeomorfismo, temos que $\varphi^{n}$ é função contínua. Assim,

$$
\lim _{k \rightarrow \infty} \varphi^{n}\left(x_{k}\right)=\varphi^{n}(y) \in Y .
$$

Então $\lambda_{Y}\left(x_{k}\right) \leq n$. Provaremos que $\lambda_{Y}\left(x_{k}\right) \rightarrow n$. Suponha que $\lambda_{Y}\left(x_{k}\right) \rightarrow$ $n^{\prime} \neq n$. Se $n^{\prime}<n$, temos que $\varphi^{n^{\prime}}\left(x_{k}\right)$ está $Y$ para todo $k$. Como $\varphi$ é contínua e $Y$ é fechado, temos que $\varphi^{n^{\prime}}(y)$ está em $Y$, o que é uma contradição.

3. Suponha que $\lambda_{Y}(Y)$ seja infinito, então existe sequência $\left\{y_{k}\right\}_{k \geq 1}$ de elementos de $Y$, tal que $\lambda_{Y}\left(y_{k}\right) \rightarrow \infty$. Como $Y$ é fechado e $X$ é compacto, temos que $Y$ é compacto. Portanto, $\left\{y_{k}\right\}_{k \geq 1}$ converge, a menos de uma subsequência, para um ponto $y_{\infty}$ em Y. Como $\lambda_{Y}$ é contínua

$$
\lim _{k \rightarrow \infty} \lambda_{Y}\left(y_{k}\right)=\lambda\left(y_{\infty}\right)=\infty
$$

o que é uma contradição. Portanto, $\lambda_{Y}(Y)$ é um conjunto finito.

Nesta seção consideraremos que $X$ é um conjunto de Cantor, $\varphi: X \rightarrow X$ um homeomorfismo minimal. Seja $Y$ um subconjunto não-vazio e clopen de $X$. Suponha que $\left\{Y_{1}, Y_{2}, \ldots, Y_{K}\right\}$ seja uma partição de $Y$ formada por conjuntos não-vazios e clopen tais que para cada $i \geq 1$, a função $\lambda_{Y}$ é constante em $Y_{i}$. Denotamos $\lambda_{Y}\left(Y_{i}\right)=J_{i}$. 
Agora, para cada $1 \leq i \leq K$ e $1 \leq j \leq J_{i}$, definimos o conjunto

$$
Y(i, j)=\varphi^{j}\left(Y_{i}\right)
$$

Lema 7.1.3. Os conjuntos

$$
\left\{Y(i, j) \mid 1 \leq i \leq K, 1 \leq j \leq J_{I}\right\}
$$

formam uma partição de $X$.

Prova. Como para cada $1 \leq i \leq K$, o conjunto $Y_{i}$ é não vazio, temos que $\varphi^{j}\left(Y_{i}\right)$ é não vazio para cada $1 \leq i, 1 \leq j \leq J_{i}$.

A função $\varphi$ é um homeomorfismo então $\varphi^{n}$ é um homeomorfismo para todo $n$ em $\mathbb{Z}$. Assim, como para cada $1 \leq i \leq K, Y_{i}$ é clopen, temos que $\varphi^{j}\left(Y_{i}\right)=Y(i, j)$ é clopen para cada $1 \leq i \leq I$ e $1 \leq j \leq J_{i}$.

Agora, mostraremos que os conjuntos $Y(i, j)$ são dois a dois disjuntos. Isto é, que,

$$
Y\left(i_{1}, j_{1}\right) \cap Y\left(i_{2}, j_{2}\right)=\emptyset \text {, para cada } 1 \leq i_{1}, i_{2} \leq I \text { e } 1 \leq j_{1}, j_{2}<J_{i_{1}},
$$

a não ser que $i_{1}=i_{2}$ e $j_{1}=j_{2}$.

É claro que se $j_{1} \neq j_{2}$ e $i_{1}=i_{1}$, temos que

$$
Y\left(i_{1}, j_{2}\right) \bigcap Y\left(i_{2}, j_{2}\right)=\emptyset
$$

Agora, suponha que $i_{1} \neq i_{2}$. Dados $1 \leq j_{1}, j_{2} \leq J_{i_{1}}$

$$
Y\left(i_{1}, j_{1}\right)=\varphi^{j_{1}}\left(Y_{i_{1}}\right) \text { e } Y\left(i_{2}, j_{2}\right)=\varphi^{j_{2}}\left(Y_{i_{2}}\right)
$$

Se $j_{1}=j_{2}$, claramente temos que $Y\left(i_{1}, j_{1}\right) \cap Y\left(i_{1}, j_{2}\right)=\emptyset$. Agora, se $j_{1} \neq j_{2}$, suponha que existem elementos $y_{i_{1}}$ e $y_{i_{2}}$ em $Y_{i_{1}}$ e $Y_{i_{2}}$, respectivamente, tais que $\varphi^{j}\left(y_{i_{1}}\right)=\varphi^{j_{2}}\left(y_{i_{2}}\right)$. Podemos supor que $j_{2}>j_{1}$, então

$$
\varphi^{j_{2}-j_{1}}\left(y_{i_{2}}\right)=y_{i_{1}} \in Y_{i_{1}}
$$

o que é uma contradição, já que $\lambda_{Y}\left(Y_{i_{1}}\right)=J_{i_{1}}>j_{2}-j_{1}$.

Portanto, só falta mostrar que

$$
\bigcup_{i=1}^{K} \bigcup_{j=1}^{J_{i}} Y(i, j)=X
$$


Temos, por definição que

$$
\mathcal{O}_{\varphi}(y) \subset \bigcup_{i=1}^{K} \bigcup_{j=1}^{J_{i}} Y(i, j)
$$

para todo $y$ em $Y$. O conjunto $\bigcup_{i=1}^{K} \bigcup_{j=1}^{J_{i}} Y(i, j)$ é fechado, já que é união finita de conjuntos fechados. Como $\varphi$ é minimal, temos que $\mathcal{O}_{\varphi}(y)$ é densa para todo $y$ em $Y$, assim

$$
X=\overline{\mathcal{O}_{\varphi}(y)}=\overline{\bigcup_{i=1}^{K} \bigcup_{j=1}^{J_{i}} Y(i, j)}=\bigcup_{i=1}^{K} \bigcup_{j=1}^{J_{i}} Y(i, j) .
$$

Concluímos, assim, a demonstração do lema.

Definição 7.1.4 (Castelo). Sejam $X$ um conjunto de Cantor, $\varphi$ um homeomorfismo minimal e $Y$ subconjunto clopen não vazio de X. Qualquer partição da forma

$$
\left\{Y(i, j) \mid 1 \leq i \leq K, 1 \leq j \leq J_{i}\right\}
$$

de X é chamada de uma partição Kakutani-Rokhlin centrada em Y ou de castelo.

Quando a partição $\left\{Y_{1}, \ldots, Y_{K}\right\}$ de $Y$ for tal que os números $J_{i}$ são dois a dois distintos para todo $1 \leq i \leq K$, o castelo é canônico e será denotado por $\mathcal{K}_{\varphi}\left(Y, Y_{1}, \ldots, Y_{k}\right)$.

Para $1 \leq i \leq K$ fixo, chamamos os conjuntos $\left\{Y(i, j) \mid 1 \leq j \leq J_{i}\right\}$ de torre.

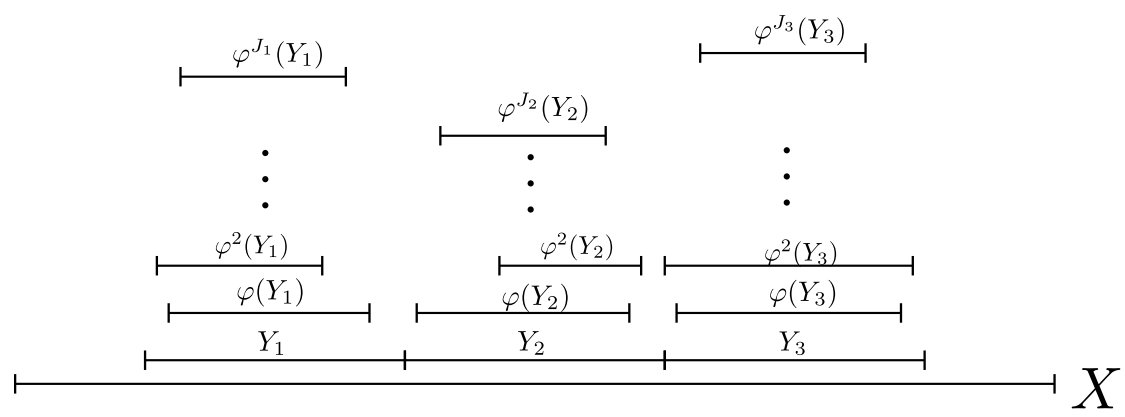

Figura 7.1: Ilustração de um castelo.

Lema 7.1.5. Sejam $X$ espaço métrico compacto e $f: X \rightarrow X$ função contínua. Para qualquer $\epsilon>0$ e $J \geq 1$, existe $\delta>0$ tal que

$$
\operatorname{diam}\left(f^{j}(B(x, \delta))\right)<\epsilon
$$

para todo $x$ em $X$ e $1 \leq j \leq J$. 
Prova. Temos que $f$ é contínua em um compacto, portanto $f$ é uniformemente contínua. Assim, dado $J \geq 1$, temos que $f^{j}$ é uniformemente contínua para todo $1 \leq j \leq J$. Então dado $\epsilon>0$, existe $\delta>0$ tal que

$$
f^{j}(B(x, \delta)) \subset B\left(f^{j}(x), \frac{\epsilon}{2}\right)
$$

para todo $x$ em $X$ e todo $1 \leq j \leq J$. Então, dados $\epsilon>0$ e $J \geq 1$, existe $\delta>0$ tal que

$$
\operatorname{diam}\left(f^{j}(B(x, \delta))\right)<\epsilon,
$$

para todo $x$ em $X$ e todo $1 \leq j \leq J$

Lema 7.1.6. Seja $X$ um conjunto de Cantor, $\varphi$ um homeomorfismo minimal e $Y$ um subconjunto clopen e não vazio de $X$. Dada uma partição $\mathcal{P}$ de $X$ existe um castelo centrado em $Y$ que é mais fino que $\mathcal{P}$.

Prova. Sejam uma partição $\mathcal{P}=\left\{X_{n}\right\}_{n \geq 1}$ de $X$ e $Y$ subconjunto clopen não vazio de $X$. Definimos

$$
\left.\epsilon=\min _{n} \operatorname{diam}\left(X_{n}\right) \quad \text { e } \quad J=\max \left\{\lambda_{Y}(Y)\right)\right\}
$$

Pelo Lema 7.1.5 existe $\delta$ tal que

$$
\operatorname{diam}\left(\varphi^{j}(B(x, \delta))<\epsilon\right.
$$

para todo $x$ em $X$ e $1 \leq j \leq J$. Consideramos uma partição $\left\{Y_{1}, \ldots, Y_{K}\right\}$ de $\mathrm{Y}$ formada por conjuntos clopen não vazios tal que para cada $1 \leq i \leq K, Y_{i}$, $\operatorname{diam}\left(Y_{i}\right)<\delta$. Então, temos que

$$
\left\{Y(i, j) \mid 1 \leq i \leq K, 1 \leq j \leq J_{i}\right\}
$$

é partição de $X$ e que

$$
\operatorname{diam}(Y(i, j))<\epsilon
$$

para todo $1 \leq i \leq K$ e $1 \leq j \leq J_{i}$.

Agora, definimos o castelo

$$
\mathcal{Q}=\left\{Y(i, j) \cap X_{N} \mid 1 \leq i \leq K, 1 \leq j \leq J_{i}, 1 \leq N \leq \#(\mathcal{P})\right\}
$$

Temos que $\mathcal{Q}$ é centrado em $Y$ e é mais fino que $\mathcal{P}$. 
Com isso mostramos que qualquer partição $\mathcal{P}$ de um conjunto de Cantor admite um castelo como partição mais fina que $\mathcal{P}$. Mas, pelo Teorema 3.1.13 que todo espaço totalmente desconexo admite uma sequência de partições refinadoras. Isso nos leva a seguinte definição:

Definição 7.1.7 (Sequência de castelos refinadores). Se para cada $n \geq 1$, $\mathcal{Q}_{n}=\left\{Y_{n}(i, j) \mid 1 \leq I_{n}, 1 \leq j \leq J_{n, i}\right\}$ é um castelo centrado em um conjunto clopen $Y_{n}$, dizemos que $\left\{\mathcal{Q}_{n}\right\}_{n \geq 1}$ é uma sequência de castelos refinadores se:

1. a sequência $\left\{\mathcal{Q}_{n}\right\}$ é uma sequência refinadora de partições;

2. para cada $n \geq 2$, os conjuntos $Y_{n}$ e $\varphi\left(Y_{n}\right)$ estão contidos em apenas um elemento de $\mathcal{Q}_{n-1}$.

3. Os conjuntos

$$
\bigcap_{n=1}^{\infty} Y_{n} e \bigcap_{n=1}^{\infty} \varphi\left(Y_{n}\right)
$$

são unitários.

Teorema 7.1.8. Sejam $X$ um conjunto de Cantor, $\varphi$ um homeomorfismo minimal em $X$ e y um elemento de $X$. Existe uma sequência de castelos refinadores centrados em conjuntos clopen $\left\{Y_{n}\right\}_{n \geq 1}$, tais que

$$
\bigcap_{n=1}^{\infty} Y_{n}=\{y\} e \bigcap_{n=1}^{\infty} \varphi\left(Y_{n}\right)=\{\varphi(y)\}
$$

Prova. Considere a sequência de conjuntos clopen

$$
Y_{0}=X \supset Y_{1} \supset Y_{2} \supset \ldots,
$$

tais que

$$
\bigcap_{n=1}^{\infty} Y_{n}=\{y\}
$$

e uma sequência de partições refinadoras $\left\{\mathcal{P}_{n}\right\}_{n \geq 1}$ de $X$. Construiremos indutivamente uma sequência de castelos $\left\{\mathcal{Q}_{n}\right\}_{n \geq 1}$, onde para cada $n \geq 1$, $\mathcal{Q}_{n}$ é centrado em $Y_{n}$ mais fino que $\mathcal{P}_{n}$.

- Etapa $n=1$ : Pelo Lema 7.1.6, existe um castelo $\mathcal{Q}_{1}$ centrado em $Y_{1}$ e mais fino que $\mathcal{P}_{1}$. Podemos escrever

$$
\mathcal{Q}_{1}=\left\{Y_{1}(i, j) \mid 1 \leq K_{1}, 1 \leq j \leq J_{1, i}\right\}
$$

- Etapa $n=2$ : Caso $Y_{2}$ não esteja contido somente em um elemento de $\mathcal{Q}_{1}$, escolhemos um conjunto clopen contido em $Y_{2}$ que contenha $y$ tal que 
isso aconteça. Agora, pelo lema 7.1.6, existe um castelo $\mathcal{Q}_{2}$ centrado em $Y_{2}$ e mais fino que a partição $\mathcal{P}_{2}$.

- Suponha que construímos castelo $\mathcal{Q}_{\ell}$ centrado em $Y_{\ell}$ e mais fino que $\mathcal{Q}_{\ell}$, tal que $Y_{\ell}$ está contido em apenas um elemento de $\mathcal{Q}_{\ell-1}$ para todo $2 \leq \ell \leq n$.

- Caso $Y_{n+1}$ não esteja contido em apenas um elemento de $\mathcal{Q}_{n}$, escolhemos um conjunto clopen contido em $Y_{n+1}$ tal que isso aconteça. Agora, pelo Lema 7.1.6, existe castelo $\mathcal{Q}_{n+1}$ centrado em $Y_{n+1}$ e mais fino que $\mathcal{P}_{n+1}$.

Temos, portanto, que a sequência $\{\mathcal{Q}\}_{n \geq 1}$ satisfaz as condições 1 e 2 da Definição 7.1.7. Mostraremos que também satisfaz a condição 3.

Como para cada $n \geq 1, Y_{n}$ é um conjunto fechado, contém $y$ e

$$
Y_{1} \supset Y_{2} \supset \ldots
$$

temos que

$$
\bigcap_{n=1}^{\infty} Y_{n}=\{y\}
$$

Além disso, temos que para cada $n \geq 1, \varphi\left(Y_{n}\right)$ é um conjunto clopen que contém $\varphi(y)$ e

$$
\varphi\left(Y_{1}\right) \supset \varphi\left(Y_{2}\right) \supset \ldots,
$$

assim

$$
\bigcap_{n=1}^{\infty} \varphi\left(Y_{n}\right)=\{\varphi(y)\}
$$

Então $\left\{\mathcal{Q}_{n}\right\}_{n \geq 1}$ também satisfaz a condição 3 e portanto é uma sequência de castelos refinadores.

\subsection{1}

\section{Construção de $\mathfrak{B}$}

Seja $\mathfrak{B}$ um diagrama de Bratteli, lembremos que na Definição 5.1.2 definimos o conjunto $X_{\mathfrak{B}}$ como o conjunto dos caminhos infinitos de $\mathfrak{B}$.

Agora, dados um conjunto de Cantor $X$ e $\varphi: X \rightarrow X$ um homeomorfismo minimal construiremos um diagrama de Bratteli simples e próprio $\mathfrak{B}=$ $(V, E, r, s, \geq)$ tal que $X_{\mathfrak{B}}$ é topologicamente conjugado a $X$.

Dada uma sequência de subconjuntos clopen $\left\{Y_{n}\right\}_{n \geq 1}$ de $X$, onde para cada $n \geq 1,\left\{Y_{n, 1}, \ldots, Y_{n, I_{n}}\right\}$ é partição de $Y_{n}$. Pelo Teorema 7.1.8, existe uma sequência $\left\{Q_{n}\right\}_{n \geq 1}$ de castelos refinadores de $X$, onde

$$
\mathcal{Q}_{n}=\left\{Y_{n}(i, j) \mid 1 \leq i \leq I_{n}, 1 \leq j \leq J_{n, i}\right\}
$$


e está centrado no conjunto $Y_{n}$.

À sequência de castelos refinadores acrescentamos o castelo trivial

$$
Q_{0}=\left\{Y_{0}(1,1)=X \mid I_{0}=1, J_{0,1}=1\right\}
$$

Estamos prontos para definir o diagrama de Bratteli

$$
\mathfrak{B}=\left(V=\left(V_{n}\right)_{n \geq 1}, E=\left(E_{n}\right)_{n \geq 1}, r, s, \geq\right) .
$$

Para cada $n \geq 1$, definimos:

$$
V_{n}=\left\{1,2, \ldots, I_{n}\right\}
$$

e

$$
\begin{gathered}
E_{n}=\left\{(\delta, i, j) \mid 1 \leq \delta \leq I_{n-1}, 1 \leq i \leq I_{n}, 1 \leq j \leq J_{n, i}\right. \\
\text { onde } \left.Y_{n}(i, j) \subset Y_{n-1}(\delta, 1)\right\}
\end{gathered}
$$

E definimos as funções $s: E_{n} \rightarrow V_{n-1}$ e $r: E_{n} \rightarrow V_{n}$ :

$$
s(\delta, i, j)=\delta \quad \text { e } \quad r(\delta, i, j)=i .
$$

Dado $n \geq 1$, denotemos por $\left(\delta_{n}, i_{n}, j_{n}\right)$ um elemento de $E_{n}$ e um elemento de $X_{\mathfrak{B}}$ por $\mathbf{x}=\left(\delta_{n}, i_{n}, j_{n}\right)_{n \geq 1}$. Assim, $r\left(\delta_{n}, i_{n}, j_{n}\right)=s\left(\delta_{n+1}, i_{n+1}, j_{n+1}\right)$ para todo $n \geq 1$, ou seja $i_{n}=\delta_{n+1}$ para todo $n \geq 1$.

Teorema 7.1.9. O diagrama de Bratteli $\mathfrak{B}$ é simples e próprio.

Prova. Mostraremos que $\mathfrak{B}$ possui todas as arestas conectadas e portanto será simples e próprio. Sejam $n \geq 1$ e $i_{n-1}$ em $V_{n-1}$. Como $\left\{\mathcal{Q}_{n}\right\}_{n \geq 0}$ é sequência de castelos refinadores, dado $i_{n}$ em $V_{n}$, existe $j_{n}$ tal que

$$
Y_{n}\left(i_{n}, j_{n}\right) \subset Y_{n-1}\left(i_{n-1}, 1\right)
$$

Então $\left(i_{n-1}, i_{n}, j_{n}\right)$ é aresta entre $i_{n-1}$ e $i_{n}$. Então $\mathfrak{B}$ possui todas as arestas conectadas.

\subsection{2}

\section{Construção da conjugação entre $X_{\mathfrak{B}}$ e $X$}

Construiremos uma conjugação entre as funções $\varphi_{\mathfrak{B}}: X_{\mathfrak{B}} \rightarrow X_{\mathfrak{B}} \mathrm{e}$ $\varphi: X \rightarrow X$. Para isso precisaremos de algumas etapas etapas preliminares. 
Lema 7.1.10. Dado $\mathbf{x}=\left(\delta_{n}, i_{n}, j_{n}\right)_{n \geq 1}$ em $X_{\mathfrak{B}}$. Considere a sequência $k_{n}=k_{n}(\mathbf{x})$ onde

$$
k_{1}=j_{1} \quad k_{n}=k_{n-1}+j_{n}-1 \quad \bmod J_{n, i_{n}} .
$$

Então para todo $n \geq 1$, temos que

$$
Y_{n+1}\left(i_{n+1}, k_{n+1}\right) \subset Y_{n}\left(i_{n}, k_{n}\right) .
$$

Observação 7.1.11. Considere $n>1$, se $k_{n-1}+j_{n}-1>J_{n, i_{n}}$ adotaremos $k_{n}=k_{n-1}+j_{n}-1 \bmod J_{n, i_{n}}$, já que dado $1 \leq \ell \leq J_{n, i_{n}}$,

$$
Y_{n}\left(i_{n}, J_{n, i_{n}}+\ell\right)=Y_{n}\left(i_{n}, \ell\right)
$$

Voltaremos, agora, a demonstração do Lema 7.1.10.

Prova. Dado $n \geq 1$, temos, por definição do conjunto $E_{n}$, que

$$
Y_{n+1}\left(i_{n+1}, j_{n+1}\right) \subset Y_{n}\left(\delta_{n+1}, 1\right) .
$$

Mas, $r\left(\delta_{n}, i_{n}, j_{n}\right)=s\left(\delta_{n+1}, i_{n+1}, j_{n+1}\right)$, portanto $\delta_{n+1}=i_{n} \mathrm{e}$

$$
Y_{n+1}\left(i_{n+1}, j_{n+1}\right) \subset Y_{n}\left(i_{n}, 1\right)
$$

Então, de acordo com a definição dos conjuntos $Y(i, j)$, temos

$$
\varphi^{j_{n+1}}\left(Y_{n+1, i_{n+1}}\right) \subset \varphi\left(Y_{n, i_{n}}\right) \Rightarrow \varphi^{j_{n+1}-1}\left(Y_{n+1, i_{n+1}}\right) \subset Y_{n, i_{n}}
$$

Portanto, para todo $k$ :

$$
\varphi^{k+j_{n+1}-1}\left(Y_{n+1, i_{n+1}}\right) \subset \varphi^{k}\left(Y_{n, i_{n}}\right)
$$

Definimos $k_{n}=k$. Assim

$$
\varphi^{k_{n}+j_{n+1}-1}\left(Y_{n+1, i_{n+1}}\right) \subset \varphi^{k_{n}}\left(Y_{n, i_{n}}\right) .
$$

mas, $k_{n}+j_{n+1}-1=k_{n+1}$, então

$$
Y_{n+1}\left(i_{n+1}, k_{n+1}\right) \subset Y_{n}\left(i_{n} \cdot k_{n}\right),
$$

provando assim o Lema.

Pelo Lema 7.1.10, $\left\{Y_{n}\left(i_{n}, k_{n}\right)\right\}_{n \geq 1}$ é uma sequência de conjuntos encai- 
xados e fechados. Além disso, $\left\{\mathcal{Q}_{n}\right\}_{n \geq 1}$ é sequência de partições refinadoras, então

$$
\operatorname{diam}\left(Y_{n}\left(\left(i_{n}, k_{n}\right)\right) \rightarrow 0\right.
$$

Portanto

$$
\bigcap_{n=1}^{\infty} Y_{n}\left(i_{n}, k_{n}\right)=\{x\} .
$$

Podemos, então, definir a função

$$
\begin{aligned}
& h: X_{\mathfrak{B}} \rightarrow X \\
& h\left(\left(\delta_{n}, i_{n}, j_{n}\right)_{n \geq 1}\right)=\bigcap_{n=1}^{\infty} Y_{n}\left(i_{n}, k_{n}\right) \\
& h\left(\mathbf{x}_{\max }\right)=\bigcap_{n=1}^{\infty} Y_{n, i_{n}} .
\end{aligned}
$$

Lema 7.1.12. A função $h: X_{\mathfrak{B}} \rightarrow X$ é um homeomorfismo.

Prova. Como $X$ é compacto, basta mostar que $h$ é uma bijeção contínua. É claro que $h$ é injetiva.

Agora, mostraremos que $h$ é sobrejetiva. Seja $x$ em $X$. Construiremos x em $X_{\mathfrak{B}}$, tal que $h(\mathbf{x})=x$.

Dado $n \geq 1$, como

$$
\left\{Y_{n}(i, j) \mid 1 \leq i \leq I_{n}, 1 \leq j \leq J_{n, i}\right\}
$$

é partição de $X$, temos que existem $i_{n}$ e $j_{n}$, tais que $x$ está em $Y_{n}\left(i_{n}, j_{n}\right)$. Como $\left\{\mathcal{Q}_{n}\right\}_{n \geq 1}$ é sequência de partições refinadoras, temos que para cada $n \geq 1$, $Y_{n}\left(i_{n}, j_{n}\right)$ está contido somente em um elemento de $\mathcal{Q}_{n-1}$. Além disso $x$ está em $Y_{n}\left(i_{n}, j_{n}\right)$ e em $Y_{n-1}\left(i_{n-1}, j_{n-1}\right)$, então

$$
Y_{n}\left(i_{n}, j_{n}\right) \subset Y_{n-1}\left(i_{n-1}, j_{n-1}\right) .
$$

$\operatorname{Como} \operatorname{diam}\left(\mathcal{Q}_{n}\right) \rightarrow 0$, temos que

$$
\bigcap Y_{n}\left(i_{n}, j_{n}\right)=\{x\} .
$$

Reescrevendo (7.1.2), temos que para todo $n \geq 1$,

$$
\varphi^{j_{n}}\left(Y_{n, i_{n}}\right) \subset \varphi^{j_{n-1}}\left(Y_{n-1, i_{n-1}}\right) .
$$

Então

$$
\varphi^{j_{n}-j_{n-1}+1}\left(Y_{n, i_{n}}\right) \subset \varphi\left(Y_{n-1, i_{n-1}}\right) .
$$

Definimos

$$
\mathbf{x}=\left(i_{n-1}, i_{n}, \ell_{n}\right)_{n \geq 1}
$$


onde

$$
\ell_{1}=j_{1} \quad \text { e } \quad \ell_{n}=j_{n}-j_{n-1}+1 \text { se } n>1
$$

Temos que

$$
h(\mathbf{x})=\bigcap Y_{n}\left(i_{n}, k_{n}\right)=\bigcap Y_{n}\left(i_{n}, j_{n}\right)=\{x\} .
$$

Falta mostrar que $h$ é contínua. Seja $\mathbf{x}=\left(\delta_{n}, i_{n}, j_{n}\right)$ em $X$. Dado $\epsilon>0$, como $\left\{\mathcal{Q}_{n}\right\}_{n \geq 1}$ é sequência de partições refinadoras, existe $n_{0}>0$, tal que $\operatorname{diam}\left(\mathcal{Q}_{n}\right)<\epsilon$ para todo $n>n_{0}$. Existe $r$ em $\mathbb{N}$, tal que $2^{-r}<\epsilon$. Definimos

$$
\delta=\min \left\{2^{-r}, 2^{-n_{0}}\right\}
$$

e seja $\mathbf{y}=\left(\lambda_{n}, \ell_{n}, p_{n}\right)$ em $X_{\mathfrak{B}}$ tal que

$$
d_{X_{\mathfrak{B}}}(\mathbf{x}, \mathbf{y})<\delta
$$

Então, para todo $N>r$,

$$
\left(\delta_{N}, i_{N}, j_{N}\right)=\left(\lambda_{N}, \ell_{N}, p_{N}\right)
$$

Portanto, para todo $N>r$ temos que $h(\mathbf{x})$ está em $Y_{N}\left(i_{N}, j_{N}\right)$ e em $Y_{N}\left(\ell_{N}, p_{N}\right)$ e além disso, temos que $\operatorname{diam}\left(Y_{n}\left(i_{n}, j_{N}\right)\right)<\epsilon$ e $\operatorname{diam}\left(Y_{n}\left(\ell_{N}, p_{N}\right)\right)<\epsilon$, então

$$
Y_{N}\left(i_{N}, j_{N}\right)=Y_{N}\left(\ell_{N}, p_{N}\right)
$$

Assim, $d_{X}(h(\mathbf{x}), h(\mathbf{y}))<\epsilon$.

Teorema 7.1.13. A função h é uma conjugação entre $\varphi_{\mathfrak{B}}$ e $\varphi$.

Prova. Como pelo Lema 7.1.12, $h$ é um homeomorfismo só falta mostrar que $h \circ \varphi_{\mathfrak{B}}=\varphi \circ h$. Para isto, mostraremos que $h^{-1} \circ \varphi=\varphi_{\mathfrak{B}} \circ h^{-1}$.

Dado $x \in X$, escrevemos

$$
\bigcap Y_{n}\left(i_{n}, j_{n}\right)=\{x\}
$$

Suponha que $N$ é a primeira entrada em que $j_{N} \neq j_{N, i_{n}}$. Iremos, então, identificar cada $J_{n, i_{n}}$ com o número 0 , já que

$$
Y_{n}\left(i_{n}, J_{n, i_{n}}\right)=Y_{n, i_{n}}
$$

Como apresentado na demonstração do Lema 7.1.12, temos que

$$
h^{-1}(x)=\left(i_{n-1}, i_{n}, \ell_{n}\right)_{n \geq 1}=\mathbf{x},
$$


onde

$$
\ell_{1}=j_{1} \quad \text { e } \quad \ell_{n}=j_{n}-j_{n-1}+1 \text { se } n>1
$$

Assim,

$$
h^{-1}(\varphi(x))=h^{-1} \varphi\left(\bigcap Y_{n}\left(i_{n}, j_{n}\right)\right) \subset h^{-1}\left(\bigcap Y_{n}\left(i_{n}, j_{n}+1\right)\right)=\left(i_{n-1}, i_{n}, \bar{\ell}_{n}\right)_{n \geq 1}
$$

Como identificamos, para todo $n, J_{n, i_{n}}$ com 0 , temos que

$$
\begin{aligned}
& \bar{\ell}_{n}=1 \quad \text { se } 1 \leq n \leq N-1, \\
& \bar{\ell}_{N}=j_{N}+1 \quad \text { e } \\
& \bar{\ell}_{n}=j_{n}-j_{n-1}+1 \quad \text { se } n>N .
\end{aligned}
$$

Assim

$$
h^{-1}(\varphi(x))=\varphi_{\mathfrak{B}}\left(\left(i_{n-1}, i_{n}, \ell_{n}\right)_{n \geq 1}\right)=\varphi_{\mathfrak{B}}\left(h^{-1}(x)\right)
$$




\section{8 \\ Relações de equivalência étale}

Nas seções anteriores, dados um conjunto de Cantor $X$, um homeomorfismo minimal $\varphi: X \rightarrow X$ e um diagrama de Bratteli $\mathfrak{B}$, estudamos dois tipos de relações de equivalência: $R_{\mathfrak{B}}$ em $X_{\mathfrak{B}}$ e $R_{\varphi}$ em $X$. Apesar delas serem relacionadas, vimos que são diferentes. O objetivo desta seção é estudar uma classe mais geral de relações de equivalência em um conjunto de Cantor $X$, onde $R_{\mathfrak{B}}$ e $R_{\varphi}$ são casos específicos.

\section{1}

\section{Ações locais e relações de equivalência Étale}

Agora, trabalharemos com espaços topológicos $\mathcal{X}=(X, \mathcal{T})$. Por motivo de conveniência denotaremos as funções $f: X \rightarrow X$ como conjuntos de pares ordenados:

$$
f=\{(x, f(x)) \mid x \in X\} .
$$

Precisamos estabelecer algumas notações. Primeiro a de composição de duas funções $f: X \rightarrow X$ e $g: X \rightarrow X$ :

$$
f \circ g=\{(x, z) \mid \text { existe } y, \quad(x, y) \in f,(y, z) \in g\} .
$$

Definição 8.1.1 (Homeomorfismo parcial). Sejam $\mathcal{X}$ e $\mathcal{Y}$ espaços topológicos. Dados $U \subset \mathcal{X}$ e $V \subset \mathcal{Y}$ subconjuntos abertos e $\gamma: U \rightarrow V$ um homeomorfismo, dizemos que $\gamma$ é um homeomorfismo parcial. Se $\mathcal{X}$ e $\mathcal{Y}$ forem totalmente desconexos, os subconjuntos $U$ e $V$ devem ser clopen.

Denotaremos por $\pi_{1}: X \times X \rightarrow X$ a projeção canônica sobre a primeira coordenada e por $\pi_{2}: X \times X \rightarrow X$ a projeção canônica sobre a segunda coordenada. Dado um conjunto aberto $U$ de $\mathcal{X}$, seja $\operatorname{id}_{U}$ a identidade definida em $U$ :

$$
\operatorname{id}_{U}=\{(x, x) \mid x \in U\}
$$

Definimos $f \cap g$, como a função $f$, definida no conjunto de pontos onde $f$ e $g$ assumem o mesmo valor.

Consideremos o espaço topológico $\mathcal{X} \times \mathcal{X}$ e os subconjuntos $A, B, C, D$ de $X$. Dadas duas funções $f: A \rightarrow B$ e $g: C \rightarrow D$, vistas como conjuntos 
podemos considerar a interseção $f \cap g$.

Seja $\mathcal{B}=\left\{U_{\alpha}\right\}_{\alpha}$ uma coleção de de subconjuntos de um espaço $X$. Dizemos que $\mathcal{B}$ é uma base para uma topologia de $X$ quando:

$-X=\bigcup_{\alpha} U_{\alpha}$.

- Dado qualquer subconjunto finito $I$ de índices, $\bigcap_{\alpha \in I} U_{\alpha} \in X$.

Definição 8.1.2 (Ação local). Seja $\mathcal{X}=(X, \mathcal{T})$ espaço topológico e $\Gamma$ uma coleção de homeomorfismos parciais de $\mathcal{X}$. A coleção $\Gamma$ é uma ação local em $\mathcal{X}$ se

1. A coleção de conjuntos $\left\{U \in \mathcal{T} \mid \operatorname{id}_{U} \in \Gamma\right\}$ forma uma base para a topologia de $X$.

2. Se $\gamma$ está em $\Gamma$ então $\gamma^{-1}$ também está.

3. Se $\gamma_{1}, \gamma_{2}$ estão em $\Gamma$ então $\gamma_{1} \circ \gamma_{2}$ também está.

4. Se $\gamma_{1}, \gamma_{2}$ estão em $\Gamma$ então $\gamma_{1} \cap \gamma_{2}$ também está.

Quando for conveniente denotaremos os elementos de $\Gamma$ por $(\gamma, U, V=\gamma(U))$, onde $U$ é o domínio de definição de $\gamma$.

Observação 8.1.3 (Interseção de funções). Se os elementos de $\Gamma$ fossem simplesmente homeomorfismos, as condições (1) (2) e (3) diriam que os elementos de $\Gamma$ formam um grupo.

Já a condição (4) nos diz que se dois homeomorfismo parciais são iguais em um ponto, então devem ser iguais em um vizinhança deste ponto. Já que $\gamma_{1} \cap \gamma_{2}$ é a função $\gamma_{1}$ definida no conjunto de pontos onde as funções $\gamma_{1}$ e $\gamma_{2}$ possuem a mesma imagem. Mas o conjunto de pontos onde duas funçôes são iguais é um conjunto fechado. Para $\gamma_{1} \cap \gamma_{2}$ ser um homeomorfismo parcial, este conjunto deve ser também aberto.

Seja $\gamma$ em $\Gamma$, associamos a $\gamma$ o aberto $U_{\gamma}$ em $\mathcal{X}$, onde $\gamma$ está definida. Assim, também associamos a $\gamma$ o aberto do produto

$$
\left\{(x, \gamma(x)) \mid x \in U_{\gamma}\right\} \subset X \times X
$$

Definimos

$$
R_{\Gamma}=\bigcup_{\gamma \in \Gamma}\left\{(x, \gamma(x)) \mid x \in U_{\gamma}\right\}
$$

Que é uma coleção de abertos em $X \times X$. Assim, dados dois pontos $x$ e $y$ em $X$, dizemos que

$$
x \sim y \text { se e somente se }(x, y) \in R_{\Gamma} .
$$


Isto é, se e somente se existe $\gamma$ em $\Gamma$ tal que $y=\gamma(x)$.

Proposição 8.1.4. Se $\Gamma$ é uma ação local no espaço $\mathcal{X}$, então $R_{\Gamma}$ é uma relação de equivalência em $X$.

Prova. Mostraremos que $R_{\Gamma}$ satisfaz as propriedades:

- Simétrica: Seja $x$ em $X$, temos que $i d: X \rightarrow X$ é um homeomorfismo parcial, assim $(x, x) \in R_{\Gamma}, \log 0 x \sim x$.

- Reflexiva: Dados $x$ e $y$ tais que $x \sim y$, então existe $\gamma \in \Gamma$, tal que $y=\gamma(x)$. Pela condição (2) da Definição 8.1.2, temos que $\gamma^{-1} \in \Gamma$. Dessa forma, $x=\gamma^{-2}(y)$ e portanto $y \sim x$.

- Transitiva: Dados $x, y$ e $z \in X$ tais que $x \sim y$ e $y \sim z$. Então existe $\gamma_{1}$ e $\gamma_{2}$ tais que

$$
y=\gamma_{1}(x) \text { e } z=\gamma_{2}(y)
$$

Temos que

$$
z=\gamma_{2}\left(\gamma_{1}(x)\right)=\gamma_{2} \circ \gamma_{1}(x)
$$

Pela condição (3) da Definição 8.1.2, temos que $\gamma_{2} \circ \gamma_{1}$ está em $\Gamma$. Portanto, $x \sim z$.

Concluímos, assim, que $R_{\Gamma}$ é uma relação de equivalência.

Repare que dado $x \in X,\langle x\rangle_{\Gamma}=\{\gamma(x) \mid \gamma \in \Gamma\}$.

Teorema 8.1.5. Se $\Gamma$ é uma ação local no espaço $\mathcal{X}$ totalmente desconexo, então $\Gamma$ é base de uma topologia em $R_{\Gamma}$, que será denotada por $\mathcal{E}_{\Gamma}$. Com a topologia $\mathcal{E}_{\Gamma}$, as funções $\pi_{1}, \pi_{2}: R_{\Gamma} \rightarrow \mathcal{X}$ são homeomorfismos locais. Neste, caso chamamos $R_{\Gamma}$ de relação de equivalência étale e $\mathcal{E}_{\Gamma}$ de topologia étale.

Prova. Pela definição de $R_{\Gamma}$ em 8.1.1, temos que os elementos de $\Gamma$ formam uma cobertura de $R_{\Gamma}$. Além, pela condição (4) da Definição 8.1.2, temos que $\Gamma$ é fechado para a interseção. Assim $\Gamma$ é base para a topologia de $R_{\Gamma}$.

O conjunto $R_{\Gamma}$ é uma coleção de abertos de $X \times X$ que podem ser escritos da forma $\gamma=(V, \gamma(V))$, onde $\gamma$ é um homeomorfismo parcial em $\Gamma$. Assim, podemos considerar a restrição de $\pi_{1}: R_{\Gamma} \rightarrow X$, ao conjunto $\gamma$ :

$$
\pi_{1_{\mid \gamma}}: \gamma=(V, \gamma(V)) \rightarrow V, \quad(x, \gamma(x)) \rightarrow x,
$$

que iremos denotar por $\pi_{1_{\gamma}}$.

Agora, mostraremos que $\pi_{1_{\gamma}}$ é um homeomorfismo local, portanto $\pi_{1}$ é um homeomorfismo parcial. 
Como $\gamma$ é homeomorfismo local e $\mathcal{X}$ é totalmente desconexo, temos que $\pi_{1}(\gamma)=V$ é um conjunto clopen e que $\pi_{1_{\gamma}}$ é uma bijeção.

Seja $U$ subconjunto clopen de $X$, tal que $i_{U}$ está em $\Gamma$. Temos que $U \cap \pi_{1}(\gamma) \subset \pi_{1}(\gamma)$ é aberto em $\pi_{1}(\gamma)$ e usando que $\pi_{1_{\gamma}}$ é bijeção obtemos que $\pi_{1}^{-1}(U \cap s(\gamma)) \subset \gamma \mathrm{e}$

$$
\pi_{1}^{-1}(U \bigcap s(\gamma))=\operatorname{id}_{U} \circ \gamma
$$

Mas, $\operatorname{id}_{U}$ e $\gamma$ estão $\Gamma$, logo pela condição (3) da Definição 8.1.2, temos que $\pi_{1}^{-1}(U \cap s(\gamma)) \subset X \times X$ está em $\Gamma$, portanto $\pi_{1}^{-1}(U \cap s(\gamma))$ é aberto em $\gamma$. Pela condição (1) da Definição 8.1.2, podemos concluir que $\pi_{1_{\gamma}}$ é contínua.

Falta mostrar que $\pi_{1_{\gamma}}^{-1}$ é contínua. Dado $\beta$ em $\Gamma$, temos que $\gamma \cap \beta$ está em $\Gamma$ e que os conjuntos $\{\beta \cap \gamma \mid \beta \in \Gamma\}$ formam uma base para a topologia relativa de $\gamma$ em $\cup \Gamma$. Temos, portanto que $\beta \cap \gamma$ é homeomorfismo local e então $\left(\pi_{1}^{-1}\right)^{-1}(\beta \bigcap \gamma)=\pi_{1}(\beta \bigcap \gamma)$ é subconjunto aberto de $\pi_{1}(\gamma)$. Concluímos que $\pi_{1_{\gamma}}^{-1}$ é contínua. A demonstração de que $\pi_{2}$ é um homeomorfismo parcial é análoga e por isso será omitida.

Corolário 8.1.6. Sejam X espaço métrico compacto e totalmente desconexo, $\Gamma$ uma ação local em $X$ e $R_{\Gamma}$ a relação de equivalência étale associada a $\Gamma$. Se $\gamma$ é aberto em $R_{\Gamma}$ e um homeomorfismo parcial, então $\gamma$ é compacto em $R_{\Gamma}$. Em particular, cada elemento de $\Gamma$ é compacto e aberto.

Prova. Como $X$ é totalmente desconexo temos que $\pi_{1}(\gamma)$ e $\pi_{2}(\gamma)$ são conjuntos clopen, em particular fechados. Como $X$ é compacto os conjuntos $\pi_{1}(\gamma)$ e $\pi_{2}(\gamma)$ são compactos. Mas, pelo Teorema 8.1.5 as funções $\pi_{1}$ e $\pi_{2}$ são homeomorfismo locais então $\gamma=(U, \gamma(U))$ é homeomorfo a $\pi_{1}(\gamma)$ e $\pi_{2}(\gamma)$. Portanto $\gamma$ é compacto.

Definição 8.1.7 (Conjunto $R$-invariante). Seja $R$ uma relação de equivalência no espaço $\mathcal{X}$. Dizemos que $A \subset X$ é $R$-invariante se para qualquer $x$ em $A$ temos que $\langle x\rangle_{R} \subset A$.

Lembre que um homeomorfismo $f: X \rightarrow X$ é minimal se e somente se os únicos fechados invariantes são $X$ e o conjunto vazio. Temos um resultado similar para relações de equivalência:

Proposição 8.1.8. Uma relação de equivalência étale $R$ associada a ação local $\Gamma$ em um conjunto de Cantor $X$ é minimal se e somente se os únicos fechados $R$-invariantes são $X$ e o conjunto vazio. 
Prova. Suponha que $R$ é minimal e que existe subconjunto $A \subset X$ tal que $A$ é fechado $R$-invariante. Dado $x$ em $A$ temos $\langle x\rangle_{R} \subset A$. Assim $\overline{\langle x\rangle_{R}} \subset \bar{A}$. Como $R$ é minimal e $A$ é fechado temos que $X=A$. Provamos, assim, a ida.

Agora, suponha que os únicos fechados $R$-invariantes são $X$ e o vazio. Dado $x$ em $X$ mostraremos que $\langle x\rangle_{R}$ é densa.

Lema 8.1.9. Seja y em $\overline{\langle x\rangle_{R}}$ e $(y, z)$ em $R$ então z está em $\overline{\langle x\rangle_{R}}$.

Seja $y$ em $\overline{\langle x\rangle_{R}}$ e $(y, z)$ em R. Provaremos que $z$ está em $\overline{\langle x\rangle_{R}}$. Como $R$ é relação de equivalência Étale, temos que existe homeomorfismo parcial $\gamma$ em $R$, tal que $(y, z)$ está em $\gamma$. Seja $\left(y_{k}\right)_{k \geq 1}$ sequência em $\overline{\langle x\rangle_{R}}$, tal que $y_{k} \rightarrow y$. Assim, existe $k_{0}$, tal que para todo $k>k_{0}, y_{k}$ está em $\pi_{1}(\gamma)$. Assim, podemos encontrar sequência $\left(y_{k}, z_{k}\right)$, tal que para todo $k>k_{0},\left(y_{k}, z_{k}\right)$ está em $\gamma$. Como $y_{k} \rightarrow y$ e $\gamma$ é contínua, temos que $z_{k} \rightarrow z$. Além disso, temos que $y_{k}$ está em $\langle x\rangle_{R}$ e $\left(y_{k}, z_{k}\right)$ está em $R$ para todo $k$, então $z_{k}$ está em $\langle x\rangle_{R}$ para todo $k$. Concluímos que $z$ está em $\overline{\langle x\rangle_{R}}$.

Pelo Lema 8.1.9, temos que $\overline{\langle x\rangle_{R}}$ é um conjunto invariante. Mas, $\overline{\langle x\rangle_{R}}$ é um conjunto fechado, como os únicos conjuntos fechados $R$-invariantes são $X$ e $\emptyset \mathrm{e}$ como $\overline{\langle x\rangle_{R}} \neq \emptyset$, temos que $\overline{\langle x\rangle_{R}}=X$. Portanto a relação de equivalência $R$ é minimal.

Definição 8.1.10. Sejam $\mathcal{X}=(X, \mathcal{T})$ e $\mathcal{Y}=(Y, \mathcal{S})$ espaços topológicos e $\Gamma$ e $\Upsilon$ ações locais em $\mathcal{X}$ e $\mathcal{Y}$, respectivamente. Dadas $R_{\Gamma}$ a relação de equivalência em $\mathcal{X}$ com topologia étale induzida por $\Gamma$ e $Q_{\Upsilon}$ a relação de equivalência em $\mathcal{Y}$ com topologia étale induzida por $\Upsilon$. Um isomorfismo entre $\left(\mathcal{X}, R_{\Gamma}, \Gamma\right)$ e $\left(\mathcal{Y}, Q_{\Upsilon}, \Upsilon\right)$ é um homeomorfismo $h: X \rightarrow Y$ tal que

$$
h \times h: R_{\Gamma} \rightarrow Q_{\Upsilon}
$$

é um homeomorfismo entre $(R, \Gamma)$ e $(Q, \Upsilon)$.

\section{2}

\section{$R_{\mathfrak{B}}$ como uma relação de equivalência étale}

Neste capítulo, mostraremos que dado um diagrama de Bratteli $\mathfrak{B}$ a relação de equivalência $R_{\mathfrak{B}}$ é uma relação de equivalência étale. Para isto, lembre a Definição 5.1.4.

Definição 8.2.1. Dado um diagrama de Bratteli $\mathfrak{B}=(V, E, r, s)$ e p e $q \in$ $E_{0, N} \operatorname{com} r(p)=r(q)$, definimos

$$
\gamma(p, q)=\left\{(\mathbf{x}, \mathbf{y}) \in X_{\mathfrak{B}} \times X_{\mathfrak{B}} \mid \mathbf{x} \in C(p), \mathbf{y} \in C(q), x_{n}=y_{n}, \forall n>N\right\} .
$$


Podemos pensar em $\gamma(p, q)$ como uma função que vai de $C(p)$ em $C(q)$ definida como segue:

$$
\gamma_{(p, q)}: C(p) \rightarrow C(q), \quad \gamma_{(p, q)}(\mathbf{x})=\left(q_{1}, \ldots, q_{N}, x_{N+1}, x_{N+2}, \ldots\right) .
$$

Proposição 8.2.2. Sejam um diagrama de Bratteli $\mathfrak{B}=(V, E, r, s), n \geq 1$ e p e q em $E_{0, N} \operatorname{com} r(p)=r(q)$. Então se verifica

1. $\gamma(p, q)^{-1}=\gamma(q, p)$.

2. $\gamma(p, q)$ é um homeomorfismo parcial entre $C(p)$ e $C(q)$.

3. $\gamma(p, p)=i d_{C(p)}$.

4. Seja b tal que $r(b)=r(q)$, então $\gamma(q, b) \circ \gamma(p, q)=\gamma(p, b)$, caso contrário é vazio.

5. Sejam a e $b$ tais que $r(a)=r(b)$ então $\gamma(p, q) \cap \gamma(a, b)=\gamma(p, q)$ se $p=a$ e $q=b$, caso contrário é vazio.

$$
\text { 6. } \gamma(p, q)=\bigcup_{e \in E_{N}+1, s(e)=r(p)=r(q)} \gamma(p e, q e) .
$$

Prova.

1. Seja x em $C(p)$, temos

$$
\begin{aligned}
\gamma_{(q, p)} \circ \gamma_{(p, q)}(\mathbf{x}) & =\gamma_{(q, p)}\left(\left(q_{1}, \ldots, q_{N}, x_{N+1}, \ldots\right)\right) \\
& =\left(p_{1}, \ldots, p_{N}, x_{N+1}, \ldots\right)=\mathbf{x} .
\end{aligned}
$$

Analogamente, mostramos que dado y em $C(q)$ temos que

$$
\gamma_{(p, q)} \circ \gamma_{(q, p)}(\mathbf{y})=\mathbf{y}
$$

Portanto, $\gamma(p, q)^{-1}=\gamma(q, p)$.

2. Pelo item anterior temos que $\gamma(p, q)$ é bijeção. Falta mostrar que $\gamma(p, q)^{-1}$ e $\gamma(q, p)$ são contínuas. Seja $\mathbf{x} \in C(p), \epsilon>0$ e $r>N$. Definimos $\delta=2^{-r}$ e consideremos $\mathbf{y}$ em $C(p)$ tal que $\mathbf{y}$ está em $B(\mathbf{x}, \delta)$, assim $x_{i}=y_{i}$ para todo $i \leq r$. Temos que

$$
\begin{gathered}
\gamma_{(p, q)}(\mathbf{x})=\left(p_{1}, \ldots p_{N}, x_{N+1}, \ldots\right) e \\
\gamma_{(p, q)}(\mathbf{y})=\left(p_{1}, \ldots, p_{N}, y_{n+1}, \ldots\right) .
\end{gathered}
$$


Mas, $x_{i}=y_{i}$ para todo $i \leq r$, então

$$
\gamma_{(p, q)}(\mathbf{y}) \in B\left(\gamma_{(p, q)}(\mathbf{x}), 2^{-r}\right) \subset B\left(\gamma_{(p, q)}(\mathbf{x}), \epsilon\right) .
$$

A demonstração de que $\gamma(p, q)^{-1}$ é contínua é análoga. Concluímos que $\gamma(p, q)$ é um homeomorfismo.

3. Segue direto da definição.

4. Reescrevendo de acordo com a notação de funções, mostraremos que

$$
\gamma_{(q, b)} \circ \gamma_{(p, q)}(\mathbf{x})=\gamma_{(p, b)}(\mathbf{x})
$$

para todo $\mathbf{x}$ em $C(p)$. Dado $\mathbf{x}$ em $C(p)$, temos que

$$
\gamma_{(q, b)} \circ \gamma_{(p, q)}(\mathbf{x})=\gamma_{(q, b)}\left(\left(q_{1}, \ldots, q_{N}, x_{N+1}, \ldots\right)\right)=\left(b_{1}, \ldots, b_{N}, x_{N+1}, \ldots\right)
$$

Por outro lado,

$$
\gamma_{(p, b)}(\mathbf{x})=\left(b_{1}, \ldots, b_{N}, x_{N+1}, \ldots\right) .
$$

Se $a \neq q$, temos que existe $N$ tal que $C(a) \neq C(q)$, mas já mostramos que quando dois cilindros são diferentes eles são disjuntos. Assim não podemos tomar a composição das funções.

5. Por definição, temos que

$$
\begin{aligned}
\gamma(p, q) \bigcap \gamma(a, b)=\{(\mathbf{x}, \mathbf{y}) \mid \mathbf{x} \in C(p) \bigcap C(a), \\
\\
\left.\quad \mathbf{y} \in C(q) \bigcap C(b), x_{n}=y_{n}, n>N\right\} .
\end{aligned}
$$

Se $p=a$ e $q=b$, temos que

$$
C(p) \bigcap C(a)=C(p) \quad \text { e } \quad C(q) \bigcap C(b)=C(q),
$$

portanto $\gamma(p, q) \cap \gamma(a, b)=\gamma(p, q)$. Agora se $p \neq a$ ou $q \neq b$, temos que

$$
C(p) \bigcap C(a)=\emptyset \quad \text { ou } \quad C(q) \bigcap C(b)=\emptyset,
$$

então $\gamma(p, q) \cap \gamma(a, b)=\emptyset$.

6. Provaremos, primeiro, que

$$
\gamma(p, q) \subset \bigcup_{e \in E_{N}+1,} \bigcup_{s(e)=r(p)=r(q)} \gamma(p e, q e) .
$$


Dado $(\mathbf{x}, \mathbf{y}) \in \gamma(p, q)$, temos que $x \in C\left(p x_{n+1}\right), y \in C\left(q y_{N+1}\right)$ e que $x_{n}=y_{n}$ para todo $n>N+1$. Mas $x_{N+1}=y_{N+1}$, então $(\mathbf{x}, \mathbf{y})$ está em $\gamma\left(p x_{N+1}, q x_{N+1}\right)$, note que $r\left(p x_{N+1}\right)=r\left(q x_{N+1}\right)$. Assim

$$
(\mathbf{x}, \mathbf{y}) \in \bigcup_{e \in E_{N}+1,} \bigcup_{s(e)=r(p)=r(q)} \gamma(p e, q e)
$$

Portanto

$$
\gamma(p, q) \subset \bigcup_{e \in E_{N}+1,} \bigcup_{s(e)=r(p)=r(q)} \gamma(p e, q e) .
$$

Agora mostraremos a inclusão contrária. Seja

$$
(\mathbf{x}, \mathbf{y}) \in \bigcup_{e \in E_{N}+1,} \bigcup_{s(e)=r(p)=r(q)} \gamma(p e, q e)
$$

Temos que existe $e$ em $E_{N+1}$ tal que $\mathbf{x}$ está em $C(p e)$, y está em $C(q e)$ e $x_{n}=y_{n}$ para todo $n>N+1$. Então $x_{N+1}=y_{N+1}=e$, portanto $(\mathbf{x}, \mathbf{y})$ está em $\gamma(p, q)$.

Concluímos assim a demonstração da proposição.

Dado um diagrama de Bratteli $\mathfrak{B}=(V, E, r, s)$, denotemos por $\Gamma_{\mathfrak{B}}$ a coleção formada pelo conjunto vazio e por todos os homeomorfismos parciais $\gamma(p, q)$, onde $p, q$ estão em $E_{0, N}$ e $N \geq 1$. Para adequação com a notação do capítulo reescrevemos $R_{\mathfrak{B}}$ da Definição 5.3.1, como:

$R_{\mathfrak{B}}=\left\{(\mathbf{x}, \mathbf{y}) \in X_{\mathfrak{B}} \times X_{\mathfrak{B}} \mid \mathbf{x}\right.$ e $\mathbf{y}$ diferem em um número finito de entradas $\}$.

Estamos prontos para provar o seguinte teorema:

Teorema 8.2.3. Seja $\mathfrak{B}=(V, E, r, s)$ um diagrama de Bratteli. A coleção $\Gamma_{\mathfrak{B}}$ é uma ação local e a relação de equivalência $R_{\mathfrak{B}}$ é gerada por $\Gamma_{\mathfrak{B}}$.

Prova. Primeiro, veremos que $\Gamma_{\mathfrak{B}}$ é uma ação local. Para isso, precisamos mostrar que $\Gamma$ satisfaz as condições da Definição 8.1.2.

1. Os cilindros $C(p)$ são uma base para $X_{\mathfrak{B}}$. Além disso, temos que $i d_{C(p)}=\gamma(p, p)$ está em $\Gamma_{\mathfrak{B}}$ para todo $p$.

2. Dados $n \geq 1$ e $p, q$ em $E_{0, N}$, seja $\gamma(p, q)$ em $\Gamma_{\mathfrak{B}}$, temos pelos itens (1) e (2) da Proposição 8.2.2 que $\gamma(p, q)^{-1}$ está em $\Gamma_{\mathfrak{B}}$.

3. Seja $n \geq 1$ e $p, q, a, b$ em $E_{0, N}$. Pelo item (4) da Proposição 8.2.2, temos que $\gamma(p, q) \circ \gamma(a, b)=\gamma(p, b)$ ou é igual ao conjunto vazio. Em qualquer um dos casos $\gamma(p, q) \circ \gamma(a, b)$ está em $\Gamma_{\mathfrak{B}}$. 
4. Seja $n \geq 1$ e $p, q, a, b$ em $E_{0, N}$. Pelo item (5), temos que $\gamma(p, q) \cap \gamma(a, b)=$ $\gamma(p, q)$ ou é igual ao conjunto vazio. Em qualquer um dos casos $\gamma(p, q) \cap \gamma(a, b)$ está em $\Gamma_{\mathfrak{B}}$.

Portanto, $\Gamma_{\mathfrak{B}}$ é uma ação local. Podemos, então, usar o Teorema 8.1.5 para afirmar que $R_{\Gamma_{\mathfrak{B}}}$ é uma relação de equivalência étale. Segue direto da definição que $R_{\Gamma_{\mathfrak{B}}}=R_{\mathfrak{B}}$.

Podemos concluir que dado diagrama de Bratteli $\mathfrak{B}=(V, E, r, s), R_{\mathfrak{B}}$ é uma relação de equivalência étale com topologia gerada pela ação local $\Gamma_{\mathfrak{B}}$, que iremos denotar por $\mathcal{T}_{\mathfrak{B}}$.

Agora veremos um exemplo onde $\mathcal{T}_{\mathfrak{B}}$ difere da topologia produto. Considere o diagrama de Bratteli $\mathfrak{B}$ apresentado na Figura 6.2 e os elementos de $X_{\mathfrak{B}}$ :

$$
\begin{aligned}
\mathbf{x} & =\mathbf{0} \\
\mathbf{y} & =(1,0,1,0,0,0, \ldots) \\
\mathbf{z}_{k} & =\left(0, \ldots, 0, z_{k}=1,0, \ldots\right) .
\end{aligned}
$$

Temos que $\mathbf{y}$ e $\mathbf{z}_{k}$ estão em $\langle\mathbf{x}\rangle_{R_{\mathfrak{B}}}$ para todo $k \geq 1$. Além disso,

$$
d\left(x, \mathbf{z}_{k}\right)=2^{1-k} \rightarrow 0 .
$$

Então $\mathbf{z}_{k} \rightarrow \mathbf{x}$, e portanto

$$
\left(\mathbf{z}_{k}, \mathbf{y}\right) \rightarrow(\mathbf{x}, \mathbf{y})
$$

na topologia produto. Veremos que $\left(\mathbf{z}_{k}, \mathbf{y}\right)$ não converge para $(\mathbf{x}, \mathbf{y})$ na topologia étale.

Sejam $p=(0,0,0)$ e $q=(1,0,1)$ em $E_{0,3}$, temos que $\left(\mathbf{z}_{k}, \mathbf{y}\right) \in \gamma(p, q)$ para todo $k \leq 3$. Entretanto, se $k \geq 4$, temos que $x_{k} \neq y_{k}$, portanto $\left(\mathbf{z}_{k}, \mathbf{y}\right)$ não está em $\gamma(p, q)$ para $k \geq 4$. Então $\left(\mathbf{z}_{k}, \mathbf{y}\right)$ não converge para $(\mathbf{x}, \mathbf{y})$ na topologia étale.

\subsection{1}

\section{Relação $A F$ em um conjunto de Cantor}

Nesta seção definiremos o que é uma relação $A F$ em um conjunto de Cantor.

Teorema 8.2.4. Considere $\mathfrak{B}=(V, E, r, s)$ um diagrama de Bratteli. $N a$ topologia étale $\mathcal{T}_{\mathfrak{B}}$ em $R_{\mathfrak{B}}$ temos que para cada $N \geq 1$, a relação de equivalência $R_{\mathfrak{B}, N}$ é aberta e compacta. 
Prova. Dado $N \geq 1$, temos que $R_{\mathfrak{B}, N}$ é a união finita de elementos de $R_{\mathfrak{B}}$. Mas cada elemento de $R_{\mathfrak{B}}$ é aberto e compacto, portanto $R_{\mathfrak{B}, N}$ também é aberto e compacto.

Definição 8.2.5 (Relação de equivalência AF). Sejam $X$ espaço topológico e $R$ uma relação de equivalência em $X$. Dizemos que $R$ é relação de equivalência AF se existe um diagrama de Bratteli $\mathfrak{B}$ tal que $(X, R)$ é isomorfo a $\left(X_{\mathfrak{B}}, R_{\mathfrak{B}}\right)$.

Teorema 8.2.6. Sejam $X$ um conjunto de Cantor e $R$ uma relação de equivalência étale em $X$. Se existe uma sequência de relações de equivalência

$$
R_{0} \subset R_{1} \subset \cdots \subset R
$$

tal que

1. $R_{N}$ é aberto e compacto em $R$ para cada $N \geq 0$,

2. $\bigcup_{N=1}^{\infty} R_{N}=R$,

então $R$ é relação de equivalência $A F$.

Prova. Construiremos o diagrama de Bratteli $\mathfrak{B}=(V, E, r, s)$, tal que $X_{\mathfrak{B}}$ será homeomorfo a $X$. Como $X$ é um conjunto de Cantor, existe uma sequência de partições refinadoras $\left\{\mathcal{P}_{n}\right\}_{n \geq 0}$ de $X$, onde para cada $n \geq 0, \mathcal{P}_{n}=\left\{U_{n, i}\right\}_{i=1}^{k(n)}$. Veja o Teorema 3.1.13.

Por hipótese, para cada $n \geq 0, R_{n}$ é compacta, então cada $R_{n}$ possui um número finito de classes de equivalência. Assim,

$$
X=\bigcup_{j=1}^{l(n)}\left\langle x_{n, j}\right\rangle_{R_{n}} .
$$

Definimos a família de conjuntos clopen $\left\{\mathcal{Q}_{n}\right\}_{n \geq 0}$, tal que para cada $n \geq 0$

$$
\mathcal{Q}_{n}=\left\{A_{n, i, j} \mid 1 \leq i \leq k(n), 1 \leq j \leq l(n)\right\}
$$

onde $A_{n, i, j}=U_{n, i} \bigcap\left\langle x_{n, j}\right\rangle_{R_{n}}$. Repare que algumas dessas interseções podem ser vazias, consideraremos somente as não vazias. Assim, para cada $n \geq 0, \mathcal{Q}_{n}$ possui um número finito de elementos.

Como $\left\{\mathcal{P}_{n}\right\}_{n \geq 0}$ é sequência de partições refinadoras de $X,\left\{Q_{n}\right\}_{n \geq 0}$ também é. Então dado $x$ em $X$, para cada $n>0$ existem únicos $i(x)_{n} \mathrm{e}$ $j(x)_{n}$, tais que $x \in A_{n, i(x)_{n}, j(x)_{n}}$. Além disso a sequência $\left\{A_{n, i(x)_{n}, j(x)_{n}}\right\}_{n \geq 0}$ é 
uma sequência de compactos encaixados. Portanto

$$
\{x\}=\bigcap_{n=1}^{\infty} A_{n, i(x)_{n}, j(x)_{n}} .
$$

Agora estamos aptos a definir os vértices e as arestas. Para cada $n \geq 0$, definimos:

$$
\begin{aligned}
& V_{n}=\left\{v=(n, i, j) \mid 1 \leq i \leq k(n), 1 \leq j \leq l(n) \text { e } A_{v}=A_{n, i, j} \neq \emptyset\right\} \\
& E_{n}=\left\{(v, w) \mid v \in V_{n}, w \in V_{n+1} \text { tais que } A_{v, n} \bigcap A_{w, n+1} \neq \emptyset\right\}
\end{aligned}
$$

Repare que $\#\left(V_{n}\right)=\#\left(\mathcal{Q}_{n}\right)$ e se $A_{v, n} \bigcap A_{w, n+1} \neq \emptyset$ então $A_{w, n+1} \subset A_{v, n}$.

Por último, definimos as funções $s: E_{n} \rightarrow V_{n-1}$ e $r: E_{n} \rightarrow V_{n}$ :

$$
s(v, w)=v \quad \text { e } \quad r(v, w)=w .
$$

Considere $v=(n, i, j) \in V_{n}$, temos que existem $i(v)_{n-1}$ e $j(v)_{n-1}$ tal que

$$
A_{n, i, j} \subset A_{n-1, i(v)_{n-1}, j(v)_{n-1}} .
$$

Portanto $r^{-1}(v) \neq \emptyset$. Analogamente, concluí-se que $s^{-1}(v) \neq \emptyset$. Portanto $\mathfrak{B}=(V, E, r, s)$ é um diagrama de Bratteli.

Temos que $\mathbf{x}=\left(v_{n}, w_{n}\right)_{n \geq 0}$ está em $X_{\mathfrak{B}}$ se e somente se para todo $n \geq 0, s\left(v_{n+1}, w_{n+1}\right)=r\left(v_{n}, w_{n}\right)$, então $v_{n+1}=w_{n}$. Portanto, podemos escrever $\mathbf{x}=\left(v_{n}, v_{n+1}\right)_{n \geq 0}$, onde cada $v_{n} \in V_{n}$.

Agora definimos a função $h: X_{\mathfrak{B}} \rightarrow X$ tal que

$$
h(\mathbf{x})=h\left(\left(v_{n}, v_{n+1}\right)_{n \geq 0}\right)=\bigcap_{n=1}^{\infty} A_{v_{n}}=\{x\} .
$$

Observação 8.2.7. A função $h$ está bem definida já que $\left\{A_{v_{n}}\right\}_{n \geq 0}$ é uma sequência de compactos encaixados.

Proposição 8.2.8. A função $h \times h$ é um homeomorfismo entre $\left(R_{\mathfrak{B}}, \mathcal{E}_{\mathfrak{B}}\right)$ e $(R, \mathcal{E})$.

Lema 8.2.9. A função $h$ é um homeomorfismo.

Prova. Pela discussão feita acima temos que $h$ é uma bijeção. Como $X_{\mathfrak{B}}$ e $X$ são compactos, veja o Teorema 5.1.6, basta mostrar que $h$ é contínua.

Sejam $\mathbf{x}=\left(x_{n}\right)_{n \geq 1}=\left(v_{n} \cdot v_{n+1}\right)_{n \geq 0}$ em $X_{\mathfrak{B}}$ e $\epsilon>0$. Existe $r$ tal que $2^{-r}<\epsilon$. Como $\left\{\mathcal{Q}_{n}\right\}$ é sequência de partições refinadoras, existe $n_{0}$ tal que para todo $n>n_{0}, \operatorname{diam}\left(\mathcal{Q}_{n}\right)<\epsilon$. Definimos $\delta=\min \left\{2^{-r}, 2^{-n_{0}}\right\}$. Dado $\mathbf{y}=\left(y_{n}\right)_{n \geq 1}=\left(w_{n}, w_{n+1}\right)_{n \geq 0}$ tal que $d_{\mathfrak{B}}(\mathbf{x}, \mathbf{y})<\delta$, temos que $x_{i}=y_{i}$ para todo 
$i \leq \max \left\{r, n_{0}\right\}, \operatorname{assim} h(\mathbf{x})$ e $h(\mathbf{y})$ estão no mesmo elemento de $\mathcal{Q}_{n}$ para todo $n<\max \left\{r, n_{0}\right\}, \log 0 d_{X}(h(\mathbf{x}), h(\mathbf{y}))<\epsilon$.

Lema 8.2.10. Para cada $N>0, h \times h\left(R_{\mathfrak{B}, N}\right)=R_{N}$.

Prova. Fixemos $N \geq 0$.

Mostraremos, primeiro, que $h \times h\left(R_{\mathfrak{B}, N}\right) \subset R_{N}$. Seja $(\mathbf{x}, \mathbf{y}) \in R_{\mathfrak{B}, N}$, temos, por definição, que $x_{i}=y_{i}$ para todo $i \geq N$. Assim, $h(\mathbf{x})$ e $h(\mathbf{y})$ estão no mesmo elemento de $\mathcal{Q}_{i}$ para todo $i \geq N$, em particular estão no mesmo elemento de $\mathcal{Q}_{N}$ e como os elementos de $\mathcal{Q}_{N}$ estão contidos em classes de equivalência, concluímos que $(h(\mathbf{x}), h(\mathbf{y})) \in R_{N}$.

Agora, provaremos que $R_{N} \subset h \times h\left(R_{\mathfrak{B}, N}\right)$. Seja $(x, y) \in R_{N}$. Existem x e $\mathbf{y}$ em $X_{\mathfrak{B}}$ tais que $h(\mathbf{x})=x$ e $h(\mathbf{y})=y$. Existe $q$ tal que $(\mathbf{x}, \mathbf{y}) \in R_{\mathfrak{B}, q}$. Veremos que $q \leq N$. Suponha que $q>n$. Temos que $x_{i}=y_{i}$ para todo $i \geq q$. Assim $x, y$ estão em $A_{q, i(x)_{q}, j(x)_{q}}$, como $\mathcal{Q}_{n}$ é sequência de partições refinadoras, temos que existem $i_{n}$ e $j_{n}$ tais que

$$
A_{q, i(x)_{q}, i(x)_{q}} \subset A_{N, i_{N}, j_{N}}=U_{N, i} \bigcap\left\langle x_{N, j}\right\rangle_{R_{N}} .
$$

Então $(\mathbf{x}, \mathbf{y}) \in A_{n, i_{n}, j_{n}}$ para todo $n \geq N$. Portanto, $(\mathbf{x}, \mathbf{y}) \in R_{\mathfrak{B}, N}$.

Concluímos assim a demonstração do lema.

Usando o lema acima temos

$$
h \times h\left(R_{\mathfrak{B}}\right)=h \times h\left(\bigcup_{n=1}^{\infty} R_{\mathfrak{B}, n}\right)=\bigcup_{n=1}^{\infty}\left(h \times h\left(R_{\mathfrak{B}, n}\right)\right)=\bigcup_{n=1}^{\infty} R_{n}=R .
$$

Portanto $h \times h$ é uma bijeção entre $R_{\mathfrak{B}}$ e $R$.

Para finalizar a demonstração do teorema falta mostrar que $h \times h\left(\mathcal{E}_{\mathfrak{B}}\right)=\mathcal{E}$.

Seja $U$ um aberto de $R$, temos que para cada $N \geq 0, U \cap R_{N}$ é um aberto em $R_{N}$. Pelo Lema 8.2.10

$$
(h \times h)^{-1}\left(U \bigcap R_{N}\right) \subset R_{\mathfrak{B}, N}
$$

Como $R_{\mathfrak{B}, N}$ é aberto em $R_{\mathfrak{B}}$, temos que $(h \times h)^{-1}\left(U \cap R_{N}\right)$ também é. Assim $\mathcal{E} \subset h \times h\left(\mathcal{E}_{\mathfrak{B}}\right)$.

Agora, dados $n \geq 0$ e $p, q$ em $E_{0, n}$, considere $\gamma(p, q)$ um aberto de $R_{\mathfrak{B}}$.

Lembre que dado um diagrama de Bratteli $\mathfrak{B}=(V, E, r, s)$ e uma sequência de inteiros $\left\{m_{k}\right\}_{k \in \mathbb{N}}$, podemos obter um novo diagrama de Bratteli $\mathfrak{T}_{m_{k}}=\mathfrak{T}=(W, F, \bar{r}, \bar{s})$. Veja Definição 5.2.1. Lembre também da função de 
blocos $f_{m_{k}}=f: X_{\mathfrak{B}} \rightarrow X_{\mathfrak{T}}$, associada à sequência $\left\{m_{k}\right\}$, definida por

$$
f\left(x_{1}, x_{2}, \ldots\right)=\left(\left(x_{1}, \ldots, x_{m_{1}}\right),\left(x_{m_{1}+1}, \ldots, x_{m_{2}}\right), \ldots\right)
$$

Lembre que denotamos $f(\mathbf{x})=\overline{\mathbf{x}}=\left(\bar{x}_{1}, \ldots, \bar{x}_{n}\right)$. Pela Proposição 5.2.2, a função $f$ é um homeomorfismo.

Teorema 8.2.11. Sejam um diagrama de Bratteli $\mathfrak{B}=(V, E, r, s)$ e $\mathfrak{T}=$ $(W, F, \bar{r}, \bar{s})$ o diagrama obtido a partir de $\mathfrak{B}$ através de uma subsequência $\left\{m_{k}\right\}_{k \in \mathbb{N}}$. A função de blocos é um isomorfismo entre $\left(X_{\mathfrak{B}}, R_{\mathfrak{B}}\right)$ e $\left(X_{\mathfrak{T}, R_{\mathfrak{T}}}\right)$.

Prova. Como $f$ é um homeomorfismo só falta mostrar que $f \times f\left(R_{\mathfrak{B}}\right)=R_{\mathfrak{T}}$ e que, $f \times f\left(\mathcal{T}_{\mathfrak{B}}\right)=\mathcal{T}_{\mathfrak{T}}$.

Para a primeira igualdade precisamos mostrar que dado $k \geq 0$,

$$
R_{\mathfrak{T}, k} \subset f \times f\left(R_{\mathfrak{B}, m}\right) \subset R_{\mathfrak{T}, k+1}, \quad \text { para todo } m_{k} \leq m \leq m_{k+1} .
$$

Assim,

$$
\bigcup_{k} R_{\mathfrak{T}, k} \subset \bigcup_{m} f \times f\left(R_{\mathfrak{B}, m}\right) \subset \bigcup_{k} R_{\mathfrak{T}, k} .
$$

Então $f \times f\left(R_{\mathfrak{B}}\right)=R_{\mathfrak{T}}$.

Agora provaremos a equação 8.2.2.

Seja $(\overline{\mathbf{x}}, \overline{\mathbf{y}}) \in R_{\mathfrak{T}, k}$. Como $f$ é homeomorfismo existem $\mathbf{x}$ e $\mathbf{y}$ em $X_{\mathfrak{B}}$ tais que $f \times f(\mathbf{x}, \mathbf{y})=(\overline{\mathbf{x}}, \overline{\mathbf{y}})$. Como $(\overline{\mathbf{x}}, \overline{\mathbf{y}})$ está em $R_{\mathfrak{T}, k}$ temos que $\overline{x_{i}}=\overline{y_{i}}$ para todo $i>k$, mas $\overline{x_{i}}=\left(x_{m_{i}-1}, \ldots, x_{m_{i}}\right)$ e $\overline{y_{i}}=\left(y_{m_{i}-1}, \ldots, y_{m_{i}}\right)$. Então $x_{\ell}=y_{\ell}$ para todo $\ell>m_{k}$ e portanto $(\mathbf{x}, \mathbf{y}) \in R_{\mathfrak{B}, m}$ para $m_{k} \leq m \leq m_{k+1}$.

Agora, seja $(\mathbf{x}, \mathbf{y})$ em $R_{\mathfrak{B}, m}$, onde $m$ é tal que $m_{k} \leq m \leq m_{k+1}$. Temos que $x_{i}=y_{i}$ para todo $i>m$, em particular para todo $i>m_{k+1}$. Então $\overline{x_{i}}=\overline{y_{i}}$ para todo $i>k+1$ então $(\overline{\mathbf{x}}, \overline{\mathbf{y}}) \in R_{\mathfrak{T}, k+1}$.

Provaremos que $f \times f\left(\mathcal{T}_{\mathfrak{B}}\right)=\mathcal{T}_{\mathfrak{T}}$. Observe que os conjuntos

$$
\left\{\gamma(\bar{p}, \bar{q}) \mid \bar{p}, \bar{q} \in F_{0, m} \text { e } r(\bar{p})=r(\bar{q})\right\}
$$

formam uma base para $\mathcal{T}_{\mathfrak{T}}$, mas se $\bar{p}, \bar{q}$ estão em $F_{0, m}$, podemos escrever

$$
\bar{p}=\left(\bar{p}_{i}\right)_{i=1}^{m} \quad \text { e } \quad \bar{q}=\left(\bar{q}_{i}\right)_{i=1}^{m}
$$

onde para cada $i \geq 1$,

$$
\bar{p}_{i}=\left(p_{j}\right)_{j=n_{i-1}+1}^{n_{i}} \quad \text { e } \quad \bar{q}_{i}=\left(q_{j}\right)_{j=n_{i-1}+1}^{n_{i}}
$$


Definimos os elementos

$$
p=\left(p_{i}\right)_{i=1}^{n_{m}} \quad \text { e } \quad q=\left(q_{i}\right)_{i=1}^{n_{m}}
$$

Então $p$ e $q$ estão em $E_{0, n_{m}}$, assim $\gamma(\bar{p}, \bar{q}) \subset \mathcal{T}_{\mathfrak{T}}$ é a imagem de $\gamma(p, q) \subset \mathcal{T}_{\mathfrak{B}}$ por $f \times f$. Portanto $\mathcal{T}_{\mathfrak{T}} \subset f \times f\left(\mathcal{T}_{\mathfrak{B}}\right)$.

Por fim, considere $f \times f(\gamma(p, q))$ em $f \times f\left(\mathcal{T}_{\mathfrak{B}}\right)$, pela condição 6 da Proposição 8.2.2, podemos escrever

$$
\gamma(p, q)=\bigcup_{\bar{p}, \bar{q} \in E_{m_{k}}} \gamma(\bar{p}, \bar{q})
$$

Assim, $f \times f(\gamma(p, q))$ pode ser escrito como a união de elementos que estão em $\mathcal{T}_{\mathfrak{T}}$.

\section{3}

\section{$R_{\varphi}$ como uma relação de equivalência étale}

Nesta seção consideraremos um espaço métrico, compacto e totalmente desconexo $X$ e um homeomorfismo $\varphi: X \rightarrow X$ sem pontos fixos. Iremos estudar a relação de equivalência $R_{\varphi}$ sob o ponto de vista topológico. Para o caso particular em que $X$ é um diagrama de Bratteli $\mathfrak{B}$ veremos a relação entre $R_{\varphi}$ e $R_{\mathfrak{B}}$

Definimos o conjunto

$$
\Gamma_{\varphi}:=\left\{\varphi_{\mid U}^{n} \mid n \in \mathbb{Z} \text { e } U \subset X \text { é clopen }\right\}
$$

Lembre que dados $x, y \in X,(x, y) \in R_{\varphi}$ se e só se existe $n$ em $\mathbb{Z}$, tal que $y=\varphi^{n}(x)$.

Teorema 8.3.1. A relação de equivalência $R_{\varphi}$ é uma relação de equivalência étale associada a ação local $\Gamma_{\varphi}$.

Lema 8.3.2. A coleção $\Gamma_{\varphi}$ é uma ação local em $X$.

Prova. Veremos primeiro que $\Gamma_{\varphi}$ satisfaz as condições da Definição 8.1.2.

1. Para todo aberto $U$ em $X$, temos que $i d_{U} \in \Gamma_{\varphi}$. Basta tomar $n=0$.

2. Dados $n \in \mathbb{Z}$, seja $\varphi_{\mid U}^{n}$ em $\Gamma_{\varphi}$, temos que $\left(\varphi_{\mid U}^{n}\right)^{-1}=\varphi_{\mid \varphi^{n}(U)}^{-n}$, que por sua vez está em $\Gamma_{\varphi}$.

3. Sejam $n_{1}, n_{2}$ em $\mathbb{Z}$ e $U_{1}, U_{2}$ subconjuntos clopen de $X$, tais que $\varphi^{n_{1}}\left(U_{1}\right) \subset$ $U_{2}$, temos que

$$
\varphi_{\mid U_{1}}^{n_{1}} \circ \varphi_{\mid U_{2}}^{n_{2}}=\varphi_{\mid U_{1}}^{n_{1}+n_{2}} \in \Gamma_{\varphi}
$$


4. Sejam $n_{1}, n_{2}$ em $\mathbb{Z}$ e $U_{1}, U_{2}$ subconjuntos clopen de $X$, temos

$$
\varphi_{\mid U_{1}}^{n_{1}} \bigcap \varphi_{\mid U 2}^{n_{2}}=\varphi_{\mid\left\{U_{1} \bigcap U_{2} \mid \varphi^{n_{1}}(x)=\varphi^{n_{2}}(x)\right\}}^{n_{1}}
$$

O conjunto $\left\{U_{1} \cap U_{2} \mid \varphi^{n_{1}}(x)=\varphi^{n_{2}}(x)\right\} \subset U_{1}$ é clopen e como $\varphi_{\mid U_{1}}^{n_{1}}$ é homeomorfismo parcial, temos que $\varphi_{\mid U_{1}}^{n_{1}} \cap \varphi_{U \mid 2}^{n_{2}}$ está em $\Gamma_{\varphi}$.

Falta ver que $\Gamma_{\varphi}$ é uma base para alguma topologia de $R_{\varphi}$. Por definição, temos que os elementos de $\Gamma_{\varphi}$ cobrem $R_{\varphi}$ e pelo item 4 concluímos que $\Gamma_{\varphi}$ é base para alguma topologia de $R_{\varphi}$. Então a relação de equivalência $R_{\varphi}$ é étale com topologia étale associada a $\Gamma_{\varphi}$.

Teorema 8.3.3. Seja $\varphi$ um homeomorfismo sem pontos fixos no espaço métrico $X$. Considere $X \times \mathbb{Z}$ com a topologia produto e $R_{\varphi}$ com topologia étale associada a ação local $\Gamma_{\varphi}$. A função

$$
h: X \times \mathbb{Z} \rightarrow R_{\varphi}, \quad h((x, n))=\left(x, \varphi^{n}(x)\right)
$$

\section{é um homeomorfismo.}

Prova. Seja $(x, y)$ em $R_{\varphi}$, existe $n \in \mathbb{Z}$ tal que $y=\varphi^{n}(x)$, então $h(x, n)=(x, y)$ e portanto $h$ é sobrejetora. Como $\varphi$ é injetora, temos que $h$ também é. Definimos, então $h^{-1}\left(\left(x, \varphi^{n}(x)\right)\right)=(x, n)$. Precisamos mostrar que $h$ e $h^{-1}$ são contínuas.

Seja $(x, n) \in X \times \mathbb{Z}$. Considere $\epsilon>0$, como $\varphi^{n}$ é contínua existe $\delta \in(0,1)$ tal que $\varphi^{n}(B(x, \delta)) \subset B\left(\varphi^{n}(x), \epsilon\right)$. Então $h(B((x, n), \delta)) \subset B(h((x, n)), \epsilon)$. Portanto $h$ é contínua.

A demonstração de que $h^{-1}$ é contínua é análoga e por isso será omitida. Concluímos que $h$ é um homeomorfismo.

Definição 8.3.4. Uma relação de equivalência étale $R$ é compactamente gerada se existe um conjunto compacto $K \subset R$ tal que se $Q$ é uma relação de equivlência e $K \subset Q \subset R$ então $Q=R$.

Proposição 8.3.5. Sejam duas relações de equivalência $(X, R)$ e $(Y, Q)$ isomorfas. Se $R$ é compactamente gerada então $Q$ também é compactamente gerada.

Prova. Como $(X, R)$ e $(Y, Q)$ são isomorfas existe homeomorfismo $h: X \rightarrow Y$ tal que $h \times h: R \rightarrow Q$ é homeomorfismo.

Seja $K \subset Q$ compacto e $Q_{1}$ outra relação de equivalência tal que $K \subset Q_{1} \subset Q$. Definimos $M \subset R_{1} \subset R$ tais que

$$
(h \times h)^{-1}(K)=M \quad \text { e } \quad(h \times h)^{-1}\left(Q_{1}\right)=R_{1},
$$


onde $M$ é compacto e $R_{1}$ é relação de equivalência. Mas, $R$ é compactamente gerada, então $R_{1}=R$, assim $Q_{1}=h \times h\left(R_{1}\right)=h \times h(R)=Q$ e portanto $Q$ é compactamente gerada.

Teorema 8.3.6. Sejam $X$ um conjunto de Cantor $e \varphi: X \rightarrow X u m$ homeomorfismo minimal. Não existe nenhum diagrama de Bratteli $\mathfrak{B}=$ $(V, E, r, s)$ tal que $\left(X, R_{\varphi}, \mathcal{E}_{\varphi}\right)$ e $\left(X_{\mathfrak{B}}, R_{\mathfrak{B}}, \mathcal{E}_{\mathfrak{B}}\right)$ são isomorfos.

Prova. Precisaremos dos seguintes lemas:

Lema 8.3.7. A relação de equivalência $R_{\varphi}$ é compactamente gerada.

Prova. Considere a função $h(x, n)=\left(x, \varphi^{-n}(x)\right)$ do Teorema 8.3.3. Como $h$ é um homeomorfismo e X é compacto, temos que $\varphi=h(X \times n)$ é compacto em $R_{\varphi}$, onde interpretamos $\varphi$ como um conjunto.

Suponha que existe relação de equivalência $Q$ tal que $\varphi \subset Q \subset R_{\varphi}$. Dado $(x, y) \in R_{\varphi}$, temos que existe $k \in \mathbb{Z}$ tal que $y=\varphi^{k}(x)$. Temos que $\left(\varphi^{n}(x), \varphi^{n+1}(x)\right) \in \varphi$ para todo $n \geq 0$, portanto $\left(\varphi^{n}(x), \varphi^{n+1}(x)\right) \in Q$ para todo $n \geq 0$. Como $Q$ é relação de equivalência temos que $(x, y) \in Q$. Então $Q=R_{\varphi}$ e $R_{\varphi}$ é compactamente gerada.

Lema 8.3.8. A relação de equivalência $R_{\mathfrak{B}}$ não é compactamente gerada.

Prova. Suponha por absurdo, que $R_{\mathfrak{B}}$ é compactamente gerada. Então existe um compacto $K$ tal que se $Q$ é relação de equivalência e $K \subset Q \subset R_{\mathfrak{B}}$ então $Q=R_{\mathfrak{B}}$. Temos que a sequência

$$
R_{\mathfrak{B}, 0} \subset R_{\mathfrak{B}, 1} \subset \ldots
$$

é uma cobertura aberta de $R_{\mathfrak{B}}$. Como $K \subset R_{\mathfrak{B}}$ também é de $K$. Assim, existe $N$ tal que $K \subset R_{\mathfrak{B}, N} \subset R_{\mathfrak{B}}$. Como, por hipótese $R_{\mathfrak{B}}$ é compactamente gerada, temos que $R_{\mathfrak{B}, N}=R_{\mathfrak{B}}$, o que é uma contradição. Concluímos que $R_{\mathfrak{B}}$ não é compactamente gerada.

Pela Proposição 8.3.5 e pelos Lemas 8.3 .7 e 8.3 .8 não existe isomorfismo entre $\left(X, R_{\varphi}\right)$ e $\left(X_{\mathfrak{B}}, R_{\mathfrak{B}}\right)$.

Repare que pelo Teorema 7.1.13, dado um conjunto de Cantor $X$ e um homeomorfismo minimal $\varphi: X \rightarrow X$, sempre existe um diagrama de Bratteli $\mathfrak{B}$ tal que $X$ é homeomorfo a $X_{\mathfrak{B}}$. Então existe um homeomorfismo $h: X \rightarrow X_{\mathfrak{B}}$. Pelo Teorema 8.3.6, a função $h \times h$ não é um homeomorfismo entre $\left(R_{\varphi}, \mathcal{E}_{\varphi}\right)$ e $\left(R_{\mathfrak{B}}, \mathcal{E}_{\mathfrak{B}}\right)$, ao contrário do que ocorreria se considerássemos a topologia produto. 


\section{9 \\ Conceitos de Álgebra}

Neste capítulo apresentaremos conceitos e resultados de álgebra que serão importantes para os próximos capítulos.

\section{1}

\section{Grupos}

Definição 9.1.1 (Grupo, subgrupo, grupo abeliano). Um grupo é um par $(G,+)$, onde $G$ é um conjunto e $\cdot$ é uma operação binária em $G$ que satisfaz as seguintes propriedades:

1. Associativa: Dados $a, b, c \in G,(a+b)+c=a+(b+c)$.

2. Elemento neutro: existe um elemento e em $G$ tal que $a+e=e+a=a$ para todo a em $G$.

3. Inverso: para cada elemento a em $G$ existe um elemento b, que chamamos de elemento inverso, tal que $a+b=b+a=e$.

Um subconjunto $H$ de $G$ é u subgrupo se é um grupo com a operação de $G$.

Um grupo é dito abeliano quando sua operação é comutativa

Quando não houver confusão denotaremos o grupo $(G,+)$ por $G$.

Em um grupo seu elemento neutro e seu elemento inverso são únicos. Denotamos o elemento inverso de $a$ em $G$ por $a^{-1}$.

Os grupos que iremos trabalhar são abelianos.

Definição 9.1.2 (Semigrupo, subsemigrupo). Um conjunto $G$ é um semigrupo se é dotado de uma operação binária associativa e possui um elemento neutro.

Um subconjunto $H$ de $G$ é dito um subsemigrupo do semigrupo $G$ se é fechado para a operação de $G$.

Observação 9.1.3. Todo grupo é um semigrupo.

Teorema 9.1.4. Sejam $G$ e $H$ grupos. Se $f: G \rightarrow H$ é um homomorfismo. Então $\operatorname{Im}(f) \cong G / \operatorname{Ker}(f)$. 


\section{2}

\section{Grupos abelianos ordenados}

Definição 9.2.1 (Cone positivo). Seja $(G,+)$ um grupo abeliano e $H$ um subconjunto de $G$. Dizemos que $H$ é o cone positivo de $G$ se satisfaz as seguintes propriedades:

1. $H$ é um subsemigrupo de $G$.

2. $H-H=\{g=a-b \mid a, b \in H\}=G$,

3. $H \cap(-H)=\{g \in G \mid g \in H e-g \in H\}=\{0\}$.

Um elemento u de $H$ é chamado de uma ordem unitária se para todo a em $G$ existe um inteiro $n$ positivo tal que $n u-a \in H$.

Definição 9.2.2 (Grupo abeliano ordenado). Seja $(G,+)$ um grupo abeliano. Se existe cone positivo $H$ de $G$, dizemos que $(G, H,+)$ é um grupo abeliano ordenado.

Se $(G, H,+)$ é um grupo abeliano ordenado, definimos

$$
a \leq b \text { se e somente se } b-a \in H .
$$

Proposição 9.2.3. Seja $(G, H,+)$ um grupo abeliano ordenado. A relação $\leq$ em (9.2.1) é uma ordem parcial em $G$.

Prova. Vamos mostrar que $\leq$ satisfaz as propriedades:

- Reflexiva: Seja $g \in G$, temos que $g-g=0 \in H$ então $g \leq g$.

- Antisimétrica: Sejam $a$ e $b$ em $G$ tais que $a \leq b$ e $b \leq a$, então $b-a \in H$ e $a-b \in H$, em particular $b-a$ e $a-b \in H \cap(-H)$. Pela condição (1) da Definição 9.2 .1 , temos que $a-b=0$ e portanto $a=b$.

- Transitividade: Sejam $a, b, c$ em $G$ tais que $a \leq b$ e $b \leq c$, então $b-a \in H$ e $c-b \in H$. Como $H$ é semigrupo, temos que

$$
(b-a)+(c-b) \in H .
$$

Como $G$ é grupo abeliano,

$$
(b-a)+(c-b)=(b-b)+(c-a)=0+(c-a)=c-a .
$$

Então $c-a \in H$ e portanto $a \leq c$.

Concluímos, assim, a demonstração da proposição. 
Corolário 9.2.4. Sejam $a, b, c$ em $G, a \leq b$ se e somente se $a+c \leq b+c$.

Prova. Temos que

$$
\begin{array}{r}
a \leq b \Leftrightarrow b-a \in H \Leftrightarrow(b-a)+(c-c) \in H \Leftrightarrow \\
(b+c)-(a+c) \in H \Leftrightarrow a+c \leq b+c
\end{array}
$$

Concluímos, assim a demonstração do corolário.

A partir de agora, iremos denotar o cone positivo de um grupo $G$ por $G^{+}$.

Um exemplo de grupo abeliano parcialmente ordenado é o grupo aditivo $(\mathbb{Z},+)$ com cone positivo $\mathbb{Z}^{+}$

Seja $(G,+)$ um grupo e considere que $A$ é um subconjunto finito de $G$. Dado $a \in A$, definimos

$$
n a=a+a+\cdots+a \text {, onde } a \text { aparece } n \text { vezes. }
$$

Definição 9.2.5 (Grupo abeliano livre). Sejam $(G,+)$ um grupo e A qualquer subconjunto finito de G. Definimos

$$
\mathbb{Z} A:=\left\{\sum_{a \in A} n_{a} a \mid n_{a} \in \mathbb{Z}, a \in A\right\}
$$

$O$ conjunto $\mathbb{Z} A$ é chamado de grupo abeliano livre gerado por $A$. Para $\mathbb{Z} A$ consideramos a operação:

$$
\sum_{a \in A} m_{a} a+\sum_{a \in A} n_{a} a=\sum_{a \in A}\left(m_{a}+n_{a}\right) a .
$$

Observamos que $(\mathbb{Z} A,+)$ é um grupo abeliano.

Proposição 9.2.6. O conjunto

$$
\mathbb{Z} A^{+}=\left\{\sum_{a \in A} n_{a} a \mid n_{a} \in \mathbb{Z}^{+} \text {para todo } a \in A\right\}
$$

é um cone positivo de $\mathbb{Z} A$.

Prova. Mostraremos que $\mathbb{Z} A^{+}$satisfaz as condições da Definição 9.2.1.

1. Segue direto da equação 9.2 .2 que $\mathbb{Z} A^{+}$é subsemigrupo de $\mathbb{Z} A$.

2. Seja $g \in \mathbb{Z} A^{+}-\mathbb{Z} A^{+}$, então $g=h_{1}-h_{2}$, onde $h_{1}$ e $h_{2}$ estão em $\mathbb{Z} A^{+}$. Então

$$
h_{1}-h_{2}=\sum_{a \in A} n_{a} a-\sum_{a \in A} m_{a} a=\sum_{a \in A}\left(n_{a}-m_{a}\right) a .
$$

Temos que $n_{a}-m_{a}$ está em $\mathbb{Z}$, portanto $h_{1}-h_{2} \in G$. 
Agora, considere $g \in \mathbb{Z} A$, então $g=\sum_{a \in A} n_{a} a$, onde $n_{a} \in \mathbb{Z}$. Temos que para cada $a \in A$, podemos escrever $n_{a}=m_{a}-p_{a}$, onde $m_{a}$ e $p_{a}$ estão em $\mathbb{Z}^{+}$. Assim,

$$
g=\sum_{a \in A} m_{a} a-\sum_{a \in A} p_{a} a \in \mathbb{Z} A^{+}-\mathbb{Z} A^{+} .
$$

3. Seja $g \in \mathbb{Z} A \cap(-\mathbb{Z} A)$, então $g=\sum_{a \in A} n_{a} a$, onde $n_{a} \in \mathbb{Z}^{+}$e $n_{a} \in-\mathbb{Z}^{+}$, então $n_{a}=0$ e portanto $g=0$.

Concluímos, assim, a demonstração da proposição.

Definição 9.2.7 (Isomorfismo ordenado). Sejam $\left(G, G^{+}\right)$e $\left(H, H^{+}\right)$grupos abelianos ordenados. Um isomorfismo ordenado entre $\left(G, G^{+}\right)$e $\left(H, H^{+}\right)$é um isomorfismo $\alpha: G \rightarrow H$, tal que $\alpha\left(G^{+}\right)=H^{+}$.

Se u é uma ordem unitária de $\left(G, G^{+}\right)$, v é uma ordem unitária de $\left(H . H^{+}\right)$, o isomorfismo $\alpha$ é dito ordenado que preserva ordem unitária se $\alpha(u)=v$. 
10

\section{O invariante $\mathrm{D}$}

Neste capítulo, associada a uma relação de equivalência étale $(X, R)$ definiremos um grupo denotado por $D(X, R)$ e mostraremos que $D(X, R)$ é um invariante algébrico. Isto é, se $(X, R)$ e $(Y, Q)$ são relações de equivalência étale isomorfas, então $D(X, R)$ e $D(Y, Q)$ também são.

\section{1}

\section{O grupo $C(X, \mathbb{Z})$}

Seja $X$ um espaço topológico compacto. Definimos o conjunto

$$
C(X, \mathbb{Z})=\{f: X \rightarrow \mathbb{Z} \mid f \text { é contínua }\}
$$

Dadas $f$ e $g$ em $C(X, \mathbb{Z})$, definimos:

$$
(f+g)(x)=f(x)+g(x), \quad x \in X
$$

Proposição 10.1.1. O par $(C(X, \mathbb{Z}),+)$ é um grupo abeliano.

Prova. Como $f(x) \in Z$ para todo $x \in X$, a operação + comuta.

Agora, mostraremos que $(C(X, \mathbb{Z}),+)$ satisfaz as propriedades da definição de grupo.

1. Sejam $f, g$ e $h$ em $C(X, \mathbb{Z})$, temos que

$$
[(f+g)+h](x)=(f+g)(x)+h(x)=(f(x)+g(x))+h(x) .
$$

Como $f(x), g(x)$ e $h(x)$ estão em $\mathbb{Z}$, temos que

$$
(f(x)+g(x))+h(x)=f(x)+(g(x)+h(x))=[f+(g+h)(x)] .
$$

2. Considere a função $\mathbf{0}(x)=0$. Mostraremos que $\mathbf{0}$ é o elemento neutro. Seja $f \in C(X, \mathbb{Z})$

$$
(\mathbf{0}+f)(x)=\mathbf{0}(x)+f(x)=0+f(x)=x=(f+\mathbf{0})(x) .
$$


3. Dada $f \in C(X, \mathbb{Z})$ provaremos que o inverso de $f$ é $-f$ :

$$
(f+(-f))(x)=f(x)+(-f)(x)=0 .
$$

Então $C(X, \mathbb{Z})$ é um grupo abeliano.

Suponha que $X$ é conexo. Dada $f$ em $C(X, \mathbb{Z})$, como $f$ é contínua, o conjunto $f(X) \subset \mathbb{Z}$ é conexo. Como os únicos conjuntos conexos de $Z$ são coonjuntos unitários a função $f$ é uma função constante, o que não é nosso interesse. De fato, $f$ é constante em cada componente conexa.

A partir de agora consideraremos que $X$ é um espaço topológico compacto e totalmente desconexo.

Dado $E \subset X$ clopen, definimos $\mathcal{X}_{E}: X \rightarrow \mathbb{Z}$ :

$$
\mathcal{X}_{E}(x)= \begin{cases}0, & x \notin E \\ 1, & x \in E\end{cases}
$$

Observação 10.1.2. A função $\mathcal{X}_{E}$ está em $C(X, \mathbb{Z})$ para todo $E \subset X$ clopen.

Seja $x \in X$ e $\epsilon>0$. Ou $x \in E$ ou $x \in X \backslash E$. Como $E$ é clopen e, $E$ e $X \backslash E$ são abertos, portanto existe $\delta>0$ tal que $B(x, \delta) \subset E$ ou $B(x, \delta) \subset X \backslash E$. Em qualquer um dos dois casos

$$
\mathcal{X}_{E}(B(x, \delta)) \subset B(x, \epsilon)
$$

Portanto, $\mathcal{X}_{E}$ é contínua.

Observação 10.1.3. Se $f \in C(X, \mathbb{Z})$ então $f(X)$ é um conjunto finito.

Seja $n \in f(X)$, o conjunto $f^{-1}(\{n\})$ é clopen, em particular aberto. Temos que

$$
X=\bigcup_{n \in f(X)} f^{-1}(\{n\}) .
$$

Como $X$ é compacto conjunto $f(X)$ tem que ser finito.

Segue direto da observação anterior que dada $f \in C(X, \mathbb{Z})$, a coleção $\left\{f^{-1}(\{n\}\}\right)_{n \in f(X)}$ é uma partição de $X$. Assim, dado $x \in X$

$$
f(x) \in f\left(\bigcup_{n \in f(X)} f^{-1}(\{n\})\right)=\bigcup_{n \in f(X)}\{n\} .
$$


Como os conjuntos $\{n\}$ são dois a dois disjuntos, existe um único $N(x)=N \in$ $f(X)$ tal que $f(x)=N$. Então, temos que

$$
f=\sum_{n \in f(X)} n \mathcal{X}_{f^{-1}(\{n\})}
$$

Pelo Teorema 3.1.13, $X$ admite uma sequência de partições refinadoras $\left\{\mathcal{P}_{n}\right\}_{n \geq 1}$, onde para cada $n \geq 1 \mathcal{P}_{n}=\left\{U_{j}\right\}_{j=1}^{k_{n}}$. Definimos, para cada $n \geq 1$, o conjunto

$$
C\left(\mathcal{P}_{n}, \mathbb{Z}\right)=\left\{f \in C(X, \mathbb{Z}) \mid f \text { é constante em cada elemento de } \mathcal{P}_{n}\right\}
$$

Temos que $C\left(\mathcal{P}_{n}, \mathbb{Z}\right)$ é um subgrupo de $C(X, \mathbb{Z})$ para todo $n \geq 1$.

Proposição 10.1.4. As seguintes afirmações são verdadeiras:

1. Para todo $n \geq 1, C\left(\mathcal{P}_{n}, \mathbb{Z}\right) \subset C\left(\mathcal{P}_{n+1}, \mathbb{Z}\right)$.

2. Para todo $n \geq 1, C\left(\mathcal{P}_{n}, \mathbb{Z}\right)$ é isomorfo a $\left(\mathbb{Z}^{k_{n}},+\right)$.

3. $\bigcup_{n=1}^{\infty} C\left(\mathcal{P}_{n}, \mathbb{Z}\right)=C(X, \mathbb{Z})$.

Prova.

1. Como $\mathcal{P}_{n+1}$ refina $\mathcal{P}_{n}$, temos que cada elemento de $\mathcal{P}_{n+1}$ está contido em algum elemento de $\mathcal{P}_{n}$. Assim se $f$ é constante em cada elemento de $\mathcal{P}_{n}$ também será constante em cada elemento de $\mathcal{P}_{n+1}$.

2. Fixe $n \geq 1$, seja $\mathcal{P}_{n}=\left\{U_{k}\right\}_{j=1}^{k_{n}}$. Definimos a função

$$
h: \mathbb{Z}^{k_{n}} \rightarrow C\left(\mathcal{P}_{n}, \mathbb{Z}\right), \quad h\left(i_{1}, \ldots, i_{k_{n}}\right):=\sum_{j=1}^{k_{n}} i_{k} \mathcal{X}_{U_{k}}
$$

Claramente $h$ é uma bijeção. Agora, considere $\left(i_{1}, \ldots, i_{k_{n}}\right)$ e $\left(l_{1}, \ldots, l_{k_{n}}\right) \in$ $\mathbb{Z}^{k_{n}}$, temos que

$$
\begin{aligned}
h\left(\left(i_{1}, \ldots, i_{k_{n}}\right)+\left(l_{1}, \ldots, l_{k_{n}}\right)\right) & =h\left(\left(i_{1}+l_{1}, \ldots, i_{k_{n}}+l_{k_{n}}\right)\right) \\
& =\sum_{j=1}^{k_{n}}\left(i_{j}+l_{j}\right) \mathcal{X}_{U_{k}} \\
& =\sum_{j=1}^{k_{n}}\left[i_{k} \mathcal{X}_{U_{j}}+l_{j} \mathcal{X}_{U_{j}}\right] \\
& =\sum_{j=1}^{k_{n}} i_{j} \mathcal{X}_{U_{j}}+\sum_{j=1}^{k_{n}} l_{j} \mathcal{X}_{U_{j}} \\
& =h\left(\left(i_{1}, \ldots, i_{k_{n}}\right)\right)+h\left(\left(l_{1}, \ldots, l_{k_{n}}\right)\right) .
\end{aligned}
$$


Portanto, $h$ é um isomorfismo entre $C\left(\mathcal{P}_{n}, \mathbb{Z}\right)$ e $\mathbb{Z}^{k_{n}}$.

3. Como para cada $n \geq 1, C\left(\mathcal{P}_{n}, \mathbb{Z}\right)$ é um subgrupo de $C(X, \mathbb{Z})$, temos que $\cup_{n=1}^{\infty} C\left(\mathcal{P}_{n}, \mathbb{Z}\right) \subset C(X, \mathbb{Z})$.

Falta provar que $C(X, \mathbb{Z}) \subset \cup C\left(\mathcal{P}_{n}, \mathbb{Z}\right)$. Par isso, precisamos mostrar que dada $f: X \rightarrow \mathbb{Z}$ em $C(X, \mathbb{Z})$, existe $n \geq 1$, tal que $f$ é constante em cada elemento de $\mathcal{P}_{n}$.

Como para cada $n \geq 1$ o conjunto $f^{-1}(\{n\})$ é clopen, podemos usar a Proposição 3.1.8, para afirmar que existe $m(n)$ tal que $f^{-1}(\{n\})$ é a união de elementos de $\mathcal{P}_{m(n)}$. Então $\mathcal{X}_{f^{-1}(\{n\})}$ está em $C\left(\mathcal{P}_{m}, \mathbb{Z}\right)$. Mas,

$$
f=\sum_{n \in f(X)} n \mathcal{X}_{f^{-1}(\{n\})}
$$

então $f \in \bigcup_{n=1}^{\infty} C\left(\mathcal{P}_{n}, \mathbb{Z}\right)$.

Concluímos que $\bigcup_{n=1}^{\infty} C\left(\mathcal{P}_{n}, \mathbb{Z}\right) \subset C(X, \mathbb{Z})$.

Finalizamos, assim, a demonstração do teorema.

\section{2}

\section{0 invariante}

Nesta seção consideraremos um espaço métrico $X$ compacto e totalmente desconexo. Agora que as ferramentas foram apresentadas, definiremos o invariante para uma relação de equivalência étale de $X$.

Seja $R$ uma relação de equivalência étale de $X$. Definimos o subgrupo $B(X, R)$ de $C(X, \mathbb{Z})$ como o subgrupo gerado por todas as funções da forma $\mathcal{X}_{\pi_{2}(\gamma)}-\mathcal{X}_{\pi_{1}(\gamma)}$, onde $\gamma \subset R$ é um homeomorfismo parcial compacto e aberto. Podemos, então, definir o grupo quociente

$$
D(X, R)=C(X, \mathbb{Z}) / B(X, R)
$$

Dada $f \in C(X, \mathbb{Z})$, denotamos sua classe em $D(X, R)$ por $\langle f\rangle$. Por último definimos

$$
D(X, R)^{+}=\{\langle f\rangle \mid f \geq 0\}
$$

Lema 10.2.1. Seja $\Gamma$ um ação local em $X$ e $R_{\Gamma}$ a relação de equivalência étale gerada por $\Gamma$. Então $B\left(X, R_{\Gamma}\right)$ é um grupo gerado por

$$
\left\{\mathcal{X}_{\pi_{2}(\gamma)}-\mathcal{X}_{\pi_{1}(\gamma)} \mid \gamma \in \Gamma\right\}
$$


Prova. Precisamos mostrar que dado $\gamma$ homeomorfismo parcial $\gamma$ compacto e aberto em $R, \mathcal{X}_{\pi_{2}(\gamma)}-\mathcal{X}_{\pi_{1}(\gamma)}$ está no subgrupo de $B\left(X, R_{\Gamma}\right)$ gerado por $\left.\mathcal{X}_{\pi_{2}(\gamma)}-\mathcal{X}_{\pi_{1}(\gamma)} \mid \gamma \in \Gamma\right\}$, que denotaremos por $H$.

Pelo Corolário 8.1.6, $\gamma$ é compacto em $R$. Como $\Gamma$ forma uma base para a topologia étale de $R$, para cada $(x, y)$ em $R$ existe uma vizinhança $\gamma_{(x, y)}$ tal que $(x, y) \in \gamma_{(x, y)} \subset \gamma$ e $\gamma=\bigcup_{(x, y) \in \gamma} \gamma_{(x, y)}$. Tais vizinhanças são apenas restrições de $\gamma$ cujos domínios podem ter interseção não vazia.

Como $\gamma$ é compacto, existe subcobertura finita, digamos $\left\{\gamma_{1}, \gamma_{2}, \ldots, \gamma_{k}\right\}$. Portanto

$$
\begin{aligned}
\mathcal{X}_{\pi_{2}(\gamma)}-\mathcal{X}_{\pi_{1}(\gamma)} & =\mathcal{X}_{\cup_{i=1}^{k} \pi_{2}\left(\gamma_{i}\right)}-\mathcal{X}_{\cup i=1}^{k} \pi_{1}\left(\gamma_{i}\right)=\mathcal{X}_{\cup_{i=1}^{k} \pi_{2}\left(\gamma_{k}\right)}-\mathcal{X}_{\cup_{i=1}^{k} \pi_{1}\left(\gamma_{k}\right)} \\
& =\sum_{1 \leq L \leq k}(-1)^{L} \sum_{i_{1}<\cdots<i_{L}} \mathcal{X}_{\pi_{2}\left(\gamma_{i_{1}} \cap \cdots \cap \pi_{1}\left(\gamma_{i_{L}}\right)\right.}-\mathcal{X}_{\pi_{1}\left(\gamma_{i_{1}} \cap \cdots \cap \pi_{1}\left(\gamma_{i_{L}}\right)\right.} \\
& =\sum_{1 \leq L \leq k}(-1)^{L} \sum_{i_{1}<\cdots<i_{L}} \mathcal{X}_{\pi_{2}\left(\gamma_{i_{1}} \cap \cdots \cap \gamma_{i_{L}}\right)}-\mathcal{X}_{\pi_{1}\left(\gamma_{i_{1}} \cap \cdots \cap \gamma_{i_{L}}\right)} \in H
\end{aligned}
$$

Concluímos, assim, a demonstração do lema.

Teorema 10.2.2. Sejam $(X, R)$ e $(Y, Q)$ relações de equivalência étale em espaços compactos e totalmente desconexos. Se $h: X \rightarrow Y$ é um homeomorfismo que define um isomorfismo entre $R$ e $Q$ então existe um isomorfismo, $\bar{g}$, entre $D(Y, Q)$ e $D(X, R)$, tal que:

1. $\bar{g}\left(D(Y, Q)^{+}\right)=D(X, R)^{+}$.

2. $\bar{g}\left(\left\langle\mathbf{1}_{Y}\right\rangle\right)=\left\langle\mathbf{1}_{X}\right\rangle$.

Lema 10.2.3. Se $h: X \rightarrow Y$ é um homeomorfismo, então a função

$$
g: C(Y, \mathbb{Z}) \rightarrow C(X, \mathbb{Z}), \quad g(f)=f \circ h
$$

é um isomorfismo entre os grupos abelianos ordenados que preserva ordem unitária.

Prova. Primeiro mostraremos que $g$ é um isomorfismo. Para isto precisamos mostrar que $g$ é um homomorfismo bijetivo.

Sejam duas funções $f_{1}$ e $f_{2}$ em $C(Y, \mathbb{Z})$ tais que $f_{1} \neq f_{2}$. Temos que

$$
g\left(f_{1}\right)=f_{1} \circ h \neq f_{2} \circ h=g\left(f_{2}\right) .
$$

Portanto $g$ é uma função injetora. Agora, seja $f$ em $C(X, \mathbb{Z})$, temos que

$$
g\left(f \circ h^{-1}\right)=\left(f \circ h^{-1}\right) \circ h=f \circ\left(h^{-1} \circ h\right)=f .
$$


Concluímos que $g$ também é uma função sobrejetora e portanto é uma bijeção. Provaremos que $g$ é um homomorfismo, sejam duas funções $f_{1}$ e $f_{2}$ em $C(Y, \mathbb{Z})$,

$$
g\left(f_{1}+f_{2}\right)=\left[f_{1}+f_{2}\right] \circ h=f_{1} \circ h+f_{2} \circ h=g\left(f_{1}\right)+g\left(f_{2}\right) .
$$

Então $g$ é um isomorfismo.

Por último, mostraremos que $g$ preserva ordem unitária. Seja $\ell$ uma ordem unitária em $C(Y, \mathbb{Z})^{+}$, então para todo $f \in C(Y, \mathbb{Z})$, existe $n$ tal que $n \ell-f \in C(Y, \mathbb{Z})^{+}$, isto é, $n \ell \leq f$. Como $h$ é homeomorfismo, para todo $y \in Y$, existe $x \in X$ tal que $h(x)=y$. Assim

$$
n \ell(y) \leq f(y) \Rightarrow n \ell(h(x)) \leq f(h(x)) \Rightarrow n(\ell \circ h)(x) \leq(f \circ h)(x) .
$$

Como $x$ é arbitrário, temos que $n(\ell \circ h) \leq f \circ h$, portanto $n g(\ell) \leq g(f)$ e $n g(\ell)-f \in C(X, \mathbb{Z})^{+}$. Concluímos que $g$ preserva ordem unitária. Finalizamos, então, a prova do lema.

Lema 10.2.4. Se h define um isomorfismo entre $R$ e $Q$, então

$$
g(B(Y, Q))=B(X, R)
$$

Prova. Como $R$ e $Q$ são relações de equivalência étale, existem ações locais $\Gamma$ e $\Delta$ que geram, respectivamente, $R$ e $Q$.

Provaremos primeiro que $g(B(Y, Q)) \subset B(X, R)$. Seja $f \in B(Y, Q)$, pelo Lema 10.2.1

$$
f=\sum_{\delta \in \Delta} n(\delta)\left(\mathcal{X}_{r(\delta)}-\mathcal{X}_{s(\delta)}\right)
$$

Então,

$$
\begin{aligned}
g(f) & =\left[\sum_{\delta \in \Delta} n(\delta)\left(\mathcal{X}_{r(\delta)}-\mathcal{X}_{s(\delta)}\right)\right] \circ g \\
& =\left[\sum_{\delta \in \Delta} n(\delta) \mathcal{X}_{r(\delta)}-\sum_{\delta \in \Delta} n(\delta) \mathcal{X}_{s(\delta)}\right] \circ g \\
& =\sum_{\delta \in \Delta} n(\delta) \mathcal{X}_{r(\delta)} \circ g-\sum_{\delta \in \Delta} n(\delta) \mathcal{X}_{s(\delta)} \circ g \\
& =\sum_{\delta \in \Delta} n(\delta) \mathcal{X}_{h^{-1}(r(\delta))}-\sum_{\delta \in \Delta} n(\delta) \mathcal{X}_{h^{-1}(s(\delta))}
\end{aligned}
$$

Definimos $\gamma:=\left(h^{-1}(r(\delta)), h^{-1}(s(\delta))\right)$, então $\gamma=(h \times h)^{-1}(\delta)$. Como $(h \times h)$ é homeomorfismo e $(h \times h)(R)=Q$, temos que $\gamma$ é um homeomorfismo parcial de $\Gamma$. Usando mais uma vez o Lema 10.2.1, temos que $g(f) \in B(X, R)$. 
Agora mostraremos que $B(X, R) \subset g(B(Y, Q))$. Como já vimos em (10.2.1), $g\left(f \circ h^{-1}\right)=f$. Demonstramos, analogamente ao que foi feito acima, que $f \circ h^{-1}$ está em $B(Y, Q)$. Portanto $B(X, R) \subset g(B(Y, Q))$.

Concluímos que $g(B(Y, Q))=B(X, R)$.

Agora, podemos terminar a demonstração do Teorema 10.2.2.

Definimos a função $\bar{g}: D(Y, Q) \rightarrow D(X, R)$ tal que

$$
\bar{g}(\langle f\rangle)=\langle g(f)\rangle
$$

Sejam $f \in C(Y, \mathbb{Z}), f_{1}$ e $f_{2}$ em $\langle f\rangle$. Temos que

$$
\left\langle f_{1}\right\rangle=\left\langle f_{2}\right\rangle \Rightarrow\left\langle f_{1}-f_{2}\right\rangle=\langle\mathbf{0}\rangle
$$

Aplicando $\bar{g}$ aos dois lados da equação (10.2.2), temos

$$
\left\langle g\left(f_{1}-f_{2}\right)\right\rangle=\langle g(\mathbf{0})\rangle
$$

Como $g$ é isomorfismo,

$$
\left\langle g\left(f_{1}\right)-g\left(f_{2}\right)\right\rangle=\langle\mathbf{0}\rangle \Rightarrow\left\langle g\left(f_{1}\right)\right\rangle=\left\langle g\left(f_{2}\right)\right\rangle .
$$

Portanto, a função $\bar{g}$ está bem definida.

Agora veremos que $\bar{g}$ é um isomorfismo. Sejam $f_{1}$ e $f_{2}$ em $C(Y, \mathbb{Z})$, segue que

$$
\bar{g}\left(f_{1}+f_{2}\right)=\left\langle g\left(f_{1}+f_{2}\right)\right\rangle .
$$

Como $g$ é isomorfismo,

$$
\left\langle g\left(f_{1}+f_{2}\right)\right\rangle=\left\langle g\left(f_{1}\right)+g\left(f_{2}\right)\right\rangle=\left\langle g\left(f_{1}\right)\right\rangle+\left\langle g\left(f_{2}\right)\right\rangle=\bar{g}\left(f_{1}\right)+\bar{g}\left(f_{2}\right) .
$$

Então $\bar{g}$ é um homomorfismo.

Falta mostrar que $\bar{g}$ é uma bijeção.

Dada $\ell$ em $D(X, R)$, como $g$ é uma sobrejeção existe $f \in C(Y, \mathbb{Z})$ tal que $g(f)=\ell$, então $\bar{g}(f)=\ell$. Portanto $g$ é uma sobrejeção.

Agora, considere $f_{1}$ e $f_{2}$ em $C(Y, \mathbb{Z})$ tais que $\bar{g}\left(f_{1}\right)=\bar{g}\left(f_{2}\right)$. Então

$$
\left\langle g\left(f_{1}\right)\right\rangle=\left\langle g\left(f_{2}\right)\right\rangle \Rightarrow\left\langle g\left(f_{1}-f_{2}\right)\right\rangle=\langle\mathbf{0}\rangle .
$$

Como $g$ é isomorfismo,

$$
\left\langle g\left(f_{1}-f_{2}\right)\right\rangle=\langle g(\mathbf{0})\rangle .
$$

Como $g(\mathbf{0})$ está em $B(X, R)$ que é igual a $g(B(Y, Q))$, temos que $f\left(f_{1}-f_{2}\right)$ está em $g(B(Y, Q))$. Assim, $f_{1}-f_{2} \in B(Y, Q)$. Portanto, como $B(Y, Q)$ é normal (é 
abeliano):

$$
\left\langle f_{1}-f_{2}\right\rangle=\langle\mathbf{0}\rangle \Rightarrow\left\langle f_{1}\right\rangle-\left\langle f_{2}\right\rangle .
$$

Concluímos que $\bar{g}$ também é uma função injetiva. Portanto é uma bijeção.

Então $\bar{g}$ é um isomorfismo. Falta mostrar que satisfaz as condições (1) e $(2)$.

1. Seja $\langle f\rangle \in D(Y, Q)^{+}$.

$$
\bar{g}(f)=\langle g(f)\rangle=\langle f \circ h\rangle .
$$

Como $\langle f\rangle \in D(Y, Q)^{+}$, temos que $f \geq 0$, então $f \circ h \geq 0$. Portanto, $\bar{g}(f) \in D(X, R)^{+}$. Provamos, então, que $\bar{g}\left(D(Y, Q)^{+}\right) \subset D(X, R)^{+}$. Mostraremos, agora, a inclusão contrária. Seja $\langle F\rangle \in D(X, R)^{+}$. Como $\bar{g}$ é sobrejetiva, existe $\langle f\rangle \in D(Y, Q)$ tal que $\bar{g}(\langle f\rangle)=\langle F\rangle$.

Afirmação 10.2.5. $\langle f\rangle \in D(Y, Q)^{+}$.

Prova. Por hipótese, $F \geq 0$. Além disso, por definição da função $\bar{g}$ temos que

$$
\langle f \circ h\rangle\langle F\rangle .
$$

Então, $f \circ h \geq 0$. Já que $h$ é um homeomorfismo, obtemos que $f \geq 0$.

Portanto $\langle F\rangle \in \bar{g}\left(D(Y, Q)^{+}\right)$. Concluímos que

$$
\bar{g}\left(D(Y, Q)^{+}\right)=D(X, R)^{+}
$$

2. Seja $1 \in C(Y, \mathbb{Z})$, temos

$$
\bar{g}(\langle\mathbf{1}\rangle)=\langle g(\mathbf{1})\rangle=\langle\mathbf{1} \circ h\rangle=\langle\mathbf{1}\rangle .
$$

Finalizamos, assim a demonstração do teorema. 
11

\section{O grupo de dimensão de um diagrama de Bratteli}

O objetivo deste capítulo é obter, a partir de um diagrama de Bratteli, um grupo abeliano ordenado. Para isto precisamos de alguns conceitos

\section{1}

\section{Limite indutivo de grupos}

Considere sequências de grupos abelianos disjuntos $\left\{G_{n}\right\}_{n \geq 0}$ e sequências de homomorfismos $\left\{\alpha_{n}\right\}_{n \geq 1}$, tais que $\alpha_{n}: G_{n-1} \rightarrow G_{n}$. Definimos

$$
\begin{gathered}
\mathbf{G}=\bigsqcup_{n} G_{n}:=\left\{(a, n) \mid n \geq 0, a \in G_{n}\right\} \quad \text { e } \\
\alpha: \bigsqcup_{n} G_{n} \rightarrow \bigsqcup_{n} G_{n}, \quad \alpha(a, n)=\left(\alpha_{n+1}(a), n+1\right) .
\end{gathered}
$$

O conjunto $\bigsqcup_{n} G_{n}$ não é um grupo.

Definição 11.1.1. Consideraremos a relação $R_{\mathbf{G}}$ em $\mathbf{G}$ dada por:

$$
(a, m) \sim_{\mathbf{G}}(b, n) \text { se e só se existem } l, k \in \mathbb{Z}^{+} \text {tais que } \alpha^{l}(a, m)=\alpha^{k}(b, n) .
$$

Em particular, $m+l=n+k$.

Proposição 11.1.2. A relação $R_{\mathbf{G}}$ é uma relação de equivalência.

Prova. Observe que as propriedades reflexiva e simétrica são imediatas. Mostraremos que também satisfaz a propriedade transitiva.

Sejam $(a, m),(b, n)$ e $(c, p)$ em $\bigsqcup_{n} G_{n}$, tais que $(a, m) \sim_{\mathbf{G}}(b, n)$ e $(b, n) \sim_{\mathbf{G}}(c, q)$. Então existem inteiros não negativos $i, j, k, l$ tais que

$$
\alpha^{i}(a, m)=\alpha^{j}(b, n) \quad \text { e } \quad \alpha^{k}(b . n)=\alpha^{l}(c, q) .
$$

Então,

$$
m+i=n+j \quad \text { e } \quad n+k=q+l .
$$

Se $k \geq j$, então

$$
m+i+k-j=n+k=q+l
$$


e portanto, $\alpha^{i+k-j}(a, m)=\alpha^{l}(c, q)$. Se $k<j$, então

$$
n+k+j-k=q+l+j-k=m+i,
$$

então $\alpha^{i}(a, m)=\alpha^{l+j-k}(c, q)$. Em ambos os casos temos que $(a, m) \sim_{\mathbf{G}}(c, q)$.

Concluímos, assim, que $R_{\mathrm{G}}$ é uma relação de equivalência.

Iremos denotar por $\langle a, m\rangle_{\mathbf{G}}$ a classe de equivalência de $(a, m)$ em $R_{\mathbf{G}}$ e por $\left(\mathbf{G}_{\infty}, \alpha_{\infty}\right)$ o conjunto de todas as classes de equivalência.

Quando não houver confusão iremos denotar a classe de equivalência de $(a, n)$ somente por $\langle a, n\rangle$.

Dado um elemento $(a, n)$ em $\mathbf{G}$, temos que $(a, n) \sim_{\mathbf{G}} \alpha^{k}(a, n)$ para todo $k \geq 0$. O elemento $\alpha^{k}(a, n)$ está em $G_{n+k} \times(n+k)$. Dessa forma, para todo $m \geq 0$, existe $(b, m)$ tal que $\langle a, n\rangle=\langle b, m\rangle$.

$\operatorname{Dados}(a, m)$ e $(b, n)$ em $\mathbf{G}$, definimos a operação + em $\left(\mathbf{G}_{\infty}, \alpha_{\infty}\right)$ por:

$$
\langle a, m\rangle_{\mathbf{G}}+\langle b, n\rangle_{\mathbf{G}}=\langle a+c, m\rangle_{\mathbf{G}}
$$

onde $c$ é tal que $\langle b, n\rangle_{\mathbf{G}}=\langle c, m\rangle_{\mathbf{G}}$.

Precisamos ver que a operação + está bem definida. Consideraremos os representantes das classes do mesmo nível. Sejam $\left(a_{1}, n\right),\left(b_{1}, n\right),\left(a_{2}, m\right)$ e $\left(b_{2}, m\right)$ em $\mathbf{G}$, tais que $\left\langle a_{1}, n\right\rangle=\left\langle a_{2}, m\right\rangle$ e $\left\langle b_{1}, n\right\rangle=\left\langle b_{2}, m\right\rangle$. Então, existem $k_{1}, k_{2}>0$ e $l_{1}, l_{2}>0$, tais que

$$
k_{1}+n=k_{2}+m, \quad l_{1}+n=l_{2}+m
$$

e

$$
\alpha^{k_{1}}\left(a_{1}, n\right)=\alpha^{k_{2}}\left(a_{2}, m\right) \quad \text { e } \quad \alpha^{l_{1}}\left(b_{1}, n\right)=\alpha^{l_{2}}\left(b_{2}, m\right) .
$$

Suponha que $k_{1} \leq l_{1}$. Então

$$
k_{2}=k_{1}+n-m \leq l_{1}+n-m=l_{2} .
$$

Temos que

$$
\begin{aligned}
\alpha^{l_{1}}\left(a_{1}, n\right) & =\alpha^{l_{1}-k_{1}} \circ \alpha^{k_{1}}\left(a_{1}, n\right)=\alpha^{l_{1}-k_{1}} \circ \alpha^{k_{2}}\left(a_{2}, m\right) \\
& =\alpha^{l_{1}-k_{1}} \circ \alpha^{k_{1}+n-m}\left(a_{2}, m\right)=\alpha^{l_{1}+n-m}\left(a_{2}, m\right) .
\end{aligned}
$$


Então,

$$
\begin{aligned}
\alpha^{l_{1}}\left(\left(a_{1}, n\right)+\left(b_{1}, n\right)\right)=\alpha^{l_{1}}\left(a_{1} . n\right)+\alpha^{l_{1}}\left(b_{1}, n\right) & =\alpha^{l_{2}}\left(a_{2}, m\right)+\alpha^{l_{2}}\left(b_{2}, m\right) \\
& =\alpha^{l_{2}}\left(\left(a_{2}, m\right)+\left(b_{2}, m\right)\right) .
\end{aligned}
$$

Portanto,

$$
\left\langle a_{1}, n\right\rangle+\left\langle b_{1}, n\right\rangle=\left\langle a_{1}+b_{1}, n\right\rangle=\left\langle a_{2}+b_{2}, m\right\rangle=\left\langle a_{2}, m\right\rangle+\left\langle b_{2}, m\right\rangle .
$$

Concluímos, assim, que a operação está bem definida.

Lema 11.1.3. O conjunto $\left(\mathbf{G}_{\infty}, \alpha_{\infty}\right)$ munido com a operação + definida acima é um grupo. Além disso, Para cada $n \geq 0$, a função $\beta_{n}: G_{n} \rightarrow\left(\mathbf{G}_{\infty}, \alpha_{\infty}\right)$, definida por

$$
\beta_{n}(a)=\langle a, n\rangle
$$

é um homomorfismo e $\beta_{n+1} \circ \alpha_{n+1}=\beta_{n}$.

Prova. Como a operação + está bem definida e como, para cada $n \geq 0, G_{n}$ é um grupo, então $\left(\mathbf{G}_{\infty}, \alpha_{\infty},+\right)$ é um grupo.

Um comentário conveniente é que dados $n$ e $m$, temos que $(0, n) \sim_{\mathbf{G}}(0, m)$. Então temos um único elemento neutro em $\left(\mathbf{G}_{\infty}, \alpha_{\infty}\right)$ e iremos representá-lo por 0 .

Seja $(a, n)$ em $G_{n}$, temos que

$$
\beta_{n+1} \circ \alpha_{n+1}(a)=\beta_{n+1}\left(\alpha_{n+1}(a), n+1\right)=\left\langle\alpha_{n+1}(a), n+1\right\rangle .
$$

Então, precisamos mostrar que $\left\langle\alpha_{n+1}(a), n+1\right\rangle=\langle a, n\rangle$. Isso acontece porque $\left(\alpha_{n+1}(a), n+1\right) \sim_{\mathbf{G}}(a, n)$.

Proposição 11.1.4. Sejam $\left\{G_{n}\right\}_{n \geq 0}$ uma sequência de grupos abelianos e $\left\{\alpha_{n}\right\}_{n \geq 1}$ uma sequência de homomorfismos, onde $\alpha_{n}: G_{n-1} \rightarrow G_{n}$. Considere $\left(\mathbf{G}_{\infty}, \alpha_{\infty}\right)$ e $\beta_{n}$ como acima. Valem as seguintes afirmações:

1. Se $\alpha_{n}$ é o homomorfismo constante igual a zero para um número infinito de indices $n$, então

$$
\left(\mathbf{G}_{\infty}, \alpha_{\infty}\right)=0
$$

2. Se existe $N \geq 0$, tal que para todo $n \geq N$, $\alpha_{n}$ é um isomorfismo então $\beta_{N}$ é um isomorfismo. 
Prova. Mostraremos, primeiro, o item (1) da proposição. Seja ( $a, n)$ em G. Por hipótese, existe $M \geq 0$ tal que $\alpha_{k}$ é o homomorfismo constante igual a 0 para todo $k \geq M$. Então, seja $k \geq M$, temos

$$
\alpha^{k}(a, n)=\left(\alpha^{k}(a), n+k\right)=(0, n+k) .
$$

Portanto, $(a, n) \sim_{\mathbf{G}}(0, n+k)$. Como $(a, n)$ é um elemento qualquer, temos

$$
\left(G_{\infty}, \alpha_{\infty}\right)=0
$$

Agora, demonstraremos o item (2) da proposição. Como $\beta_{N}$ é homomorfismo, falta mostrar que é bijeção.

Provaremos que $\beta_{n}$ é injetiva. Sejam $a$ e $b$ em $G_{N}$, tais que $a \neq b$. Suponha, por absurdo que $\beta_{N}(a)=\beta_{N}(b)$, então $(a, N) \sim_{\mathbf{G}}(b, N)$. Portanto existe inteiro $k \geq 0$ tal que

$$
\alpha^{k}(a)=\alpha^{k}(b)
$$

então, como $\alpha_{i}$ é injetiva para todo $i \geq K$, temos que $a=b$, o que é uma contradição.

Por último, precisamos mostrar que $\beta_{N}$ é sobrejetiva.

Lema 11.1.5. Para todo $k \geq 1$,

$$
\beta_{N}\left(G_{N}\right)=\beta_{N+k}\left(G_{N+k}\right) .
$$

Prova. Seja $g_{N+1} \in G_{N+1}$, temos que como $\alpha_{N+1}$ é sobrejeção, existe $g_{n} \in G_{N}$ tal que

$$
\alpha_{N+1}\left(g_{N}\right)=g_{N+1} \text {. }
$$

Então,

$$
\beta_{N+1}\left(g_{N+1}\right)=\beta_{N+1}\left(\alpha_{N+1}\left(g_{N+1}\right)\right)=\beta_{N}\left(g_{N}\right) \in \beta_{N}\left(G_{N}\right) .
$$

Portanto, $\beta_{N+1}\left(G_{N+1}\right) \subset \beta_{N}\left(G_{N}\right)$. Indutivamente, provamos que para todo $k \geq 0, \beta_{N+K}\left(G_{N+k}\right) \subset \beta_{N}\left(G_{N}\right)$.

Mostraremos a inclusão contrária. Dado $k \geq 0$, definimos

$$
\alpha_{N}^{K}:=\alpha_{N+K} \circ \ldots \alpha_{N+1} .
$$

Seja $g \in \beta_{N}\left(G_{N}\right)$, existe $g_{N} \in G_{N}$ tal que $\beta_{n}\left(g_{n}\right)=g$. Mas, pela definição da função $\beta_{N}$, segue que

$$
g=\beta_{N}\left(g_{N}\right)=\beta_{N+k} \circ \alpha_{N}^{k}\left(g_{n}\right) \in \beta_{N+k}\left(G_{N+k}\right)
$$


Provamos, então, que para todo $k \geq 0$,

$$
\beta_{N}\left(G_{N}\right)=\beta_{N+k}\left(G_{N+k}\right)
$$

Agora, mostraremos que,

$$
\left(\mathbf{G}_{\infty}, \alpha_{\infty}\right)=\beta_{N}\left(G_{N}\right)
$$

Seja $\langle a, n\rangle \in\left(\mathbf{G}_{\infty}, \alpha_{\infty}\right)$. Existe $m>N$ tal que $\langle a, n\rangle \in B_{m}\left(G_{m}\right)$. Pelo Lema 11.1.5, concluímos que $\langle a, n\rangle \in B_{N}\left(G_{N}\right)$ e, portanto, $\beta_{N}$ é uma função sobrejetiva. Como $\beta_{N}$ é uma bijeção e um homomorfismo é um isomorfismo.

Seja $G$ um grupo abeliano e $\left\{G_{n}\right\}_{n \geq 0}$ uma sequência de subgrupos de $G$ tal que $G_{n} \subset G_{n+1}$ e cuja união é o grupo $G$. Considere que, para todo $n \geq 0$, $\alpha_{n+1}$ é a inclusão de $G_{n}$ em $G_{n+1}$. Definimos a função

$$
\varphi: G \rightarrow\left(G_{\infty}, \alpha_{\infty}\right), \quad \varphi(a)=\langle a, n\rangle \text {, onde } n \text { menor número tal que } a \in G_{n} \text {. }
$$

que está claramente bem definida. Isto é, independe da escolha do índice $n$.

Proposição 11.1.6. A função $\varphi$ é um isomorfismo.

Prova. Mostraremos, primeiro, que $\varphi$ é um homomorfismo. Sejam $a$ e $b$ em $G$. Como a sequência $\left\{G_{n}\right\}_{n \geq 0}$ é encaixada e $\bigcup_{n} G_{n}=G$, existe $m$ tal que $G_{m}$ é o primeiro elemento que contém $a$ e existe $n$, tal que $G_{n}$ é o primeiro elemento que contém $b$. Se $m \leq n$, então $G_{n}$ é o primeiro elemento que contém $a+b$. Além disso, como para todo $m \geq 0, \alpha_{m+1}$ é a inclusão de $G_{m}$ em $G_{m+1}$, temos que

$$
\langle a, m\rangle=\langle a, k\rangle, \text { para todo } k \geq m \text {. }
$$

Portanto,

$$
\varphi(a+b)=\langle a+b, n\rangle=\langle a, n\rangle+\langle b, n\rangle=\varphi(a)+\varphi(b) .
$$

Claramente, $\varphi$ é sobrejetiva. Para mostrar que $\varphi$ é injetiva, suponha que $\varphi(a)=\varphi(b)$. Tomando $m, n$ como acima, temos que

$$
\varphi(a)=\langle a, m\rangle=\langle a, n\rangle=\langle b, n\rangle=\varphi(b) .
$$

Então, existem inteiros $k$ e $\ell$ tais que

$$
\alpha_{n}^{k}(a)=\alpha_{n}^{k}(b)
$$


Portanto, $k=\ell$. Como $\alpha_{n}$ é a inclusão e $\alpha_{n}^{k}(a)=\alpha_{n}^{k}(b)$, concluímos que $a=b$. Mostramos, assim, que $\varphi$ é injetiva. Portanto $\varphi$ é uma bijeção. Concluímos, assim, que $\varphi$ é um isomorfismo.

Agora, considere para cada $n \geq 0$, um subgrupo $H_{n}$ de $G_{n}$ tal que $H_{n} \subset H_{n+1}$. Seja $H=\bigcup_{n} H_{n}$ Definimos as funções:

$$
\widetilde{\alpha}_{n}: G_{n} / H_{n} \rightarrow G_{n+1} / H_{n+1}, \quad \widetilde{\alpha}_{n}\left(a+H_{n}\right)=a+H_{n+1}, \quad a \in G_{n}
$$

$\mathrm{e}$

$$
\begin{aligned}
& \phi: G / H \rightarrow\left((\mathbf{G} / H)_{\infty}, \widetilde{\alpha}_{\infty}\right) \\
& \phi(a+H)=\left\langle a+H_{n}, n\right\rangle, n \text { menor número tal que } a \in G_{n} .
\end{aligned}
$$

A função $\widetilde{\alpha}_{n}$, para todo $n \geq 0$ está claramente bem definida.

Proposição 11.1.7. A função $\phi$ é um isomorfismo.

Prova. Temos que mostrar que $\phi$ é um homomorfismo bijetivo. Primeiro provaremos que é homomorfismo. Sejam $a$ e $b$ em $G$, existe $m \geq 0$, tal que $G_{m}$ é o primeiro elemento que contém $a$ e existe $n$ tal que $G_{n}$ é o primeiro elemento que contém $b$. Suponha que $n \geq m, G_{n}$ é o primeiro elemento que contém $a+b$. Além disso, pela definição da função $\tilde{\alpha}_{n}$, temos que

$$
\left\langle a+H_{m}, m\right\rangle=\left\langle a+H_{k}, k\right\rangle, \text { para todo } k \geq m .
$$

Então

$$
\begin{aligned}
\phi((a+H)+(b+H)) & =\phi((a+b)+H) \\
& =\left\langle(a+b)+H_{n}, n\right\rangle=\left\langle\left(a+H_{n}\right)+\left(b+H_{n}\right), n\right\rangle \\
& =\left\langle a+H_{n}, n\right\rangle+\left\langle b+H_{n}, n\right\rangle=\phi(a+H)+\phi(b+H) .
\end{aligned}
$$

Claramente $\phi$ é uma sobrejeção. Falta, então, mostrar que $\phi$ é injetiva. Sejam $a$ e $b$ em $G$ tais que $a \in G_{n}, b \in G_{k}$ e $\phi(a+H)=\phi(b+H)$. Suponha que $n \geq k$, então

$$
\left\langle a+H_{n}, n\right\rangle=\left\langle b+H_{k}, k\right\rangle .
$$

Portanto, existem inteiros $l, m \geq 0$, tais que

$$
\widetilde{\alpha}^{l}\left(\left(a+H_{n}, n\right)\right)=\widetilde{\alpha}^{m}\left(\left(b+H_{k} \cdot k\right)\right) \quad \text { e } \quad n+l=k+m .
$$


Definimos $M:=n+l=m+k$, temos que

$$
a+H_{M}=b+H_{M}
$$

Como a sequência de subgrupos é encaixada e a união é $H$, segue que

$$
a+H=b+H .
$$

Portanto $\phi$ é injetiva.

Logo, $\phi$ é um isomorfismo.

Definição 11.1.8 (Homomorfismo positivo). Sejam $\left(G, G^{+}\right)$e $\left(H, H^{+}\right)$grupos abelianos ordenados. Um homomorfismo $\varphi: G \rightarrow H$ é positivo se $\varphi\left(G^{+}\right) \subset H^{+}$.

Teorema 11.1.9. Sejam $\left\{\left(G_{n}, G_{n}^{+}\right)\right\}_{n \geq 0}$ uma sequência de grupos abelianos ordenados e $\left\{\alpha_{n}\right\}_{n \geq 0}$ uma sequência de homomorfismos positivos, onde $\alpha_{n}$ : $G_{n} \rightarrow G_{n+1}$. Então

$$
\left\{\langle a, n\rangle \mid n \geq 0, a \in G_{n}^{+}\right\}
$$

é o cone positivo de $\left(G_{\infty}, \alpha_{\infty}\right)$.

Prova. Denotaremos o conjunto $\left\{\langle a, n\rangle \mid n \geq 0, a \in G_{n}^{+}\right\}$por $H$. Temos que mostrar que $H$ satisfaz as condições da Definição 9.2.1 de cone positivo.

1. Como, para cada $n \geq 0, G_{n}^{+}$é um cone positivo, temos que $H$ é um subsemigrupo de $\left(G_{\infty}, \alpha_{\infty}\right)$.

2. Precisamos ver que $H-H=\left(\mathbf{G}_{\infty}, \alpha_{\infty}\right)$. É claro que $H-H \subset\left(\mathbf{G}_{\infty}, \alpha_{\infty}\right)$ Agora, considere $\langle a, n\rangle \in\left(G_{\infty}, \alpha_{\infty}\right)$. Como $G_{n}^{+}$é cone positivo, existem $b$ e $c$ em $G_{n}^{+}$tais que $a=b-c$. Então $\langle b-c, n\rangle \in H-H$.

3. Mostraremos que $H \bigcap(-H)=\{0\}$. Seja $\langle a, n\rangle \in H \bigcap(-H)$. Temos que $\langle a, n\rangle \in H$ e $\langle-a, n\rangle \in H$. Então $a$ e $-a$ estão em $G_{n}^{+}$. Como $G_{n}^{+}$é cone positivo, $a=0$. Portanto,

$$
\langle a, n\rangle=\langle 0, n\rangle=0
$$

Concluímos que $H \cap(-H)=\{0\}$.

Mostramos, assim, que $H$ é o cone positivo de $\left(G_{\infty}, \alpha_{\infty}\right)$. A partir de agora, denotaremos $H$ por $\left(G_{\infty}, \alpha_{\infty}\right)^{+}$. 


\section{2}

\section{O grupo de dimensão de um diagrama de Bratteli}

$\mathrm{Na}$ definição de um diagrama de Bratteli

$$
\mathfrak{B}=\left(V=\left\{V_{n}\right\}_{n}, E, r, s\right)
$$

o conjunto $V_{0}$ era unitário. Tal restrição não será necessária no momento. Portanto, nesta seção o conjunto $V_{0}$ pode ter mais de um elemento.

Iremos obter a partir do diagrama de Bratteli $\mathfrak{B}$ um grupo abeliano ordenado que será a união de uma sequência de grupos abelianos disjuntos, como na seção anterior.

\subsection{1}

A função $h_{\mathfrak{B}_{n}}$

Seja $\mathfrak{B}=\left(V=\left\{V_{n}\right\}_{n \geq 0}, E, r, s\right)$ um diagrama de Bratteli. Definimos, para cada $n \geq 0$, o grupo $G_{n}=\mathbb{Z} V_{n}$, veja a Definição 9.2 .5 , que possui $\mathbb{Z} V_{n}^{+}$ como cone positivo, veja a Proposição 9.2.6. Dessa forma, um elemento $g$ de $\mathbb{Z} V_{n-1}$, pode ser expresso como

$$
g=\sum_{i=1}^{\# V_{n-1}} m_{i} v_{i}^{n-1}, \quad \text { onde } v_{i}^{n-1} \in V_{n-1} \text { e } m_{i} \in \mathbb{Z} .
$$

Fixe $n \geq 1$ e considere a função $h_{\mathfrak{B}_{n}}: \mathbb{Z} V_{n-1} \rightarrow \mathbb{Z} V_{n}$ definida por:

$$
h_{\mathfrak{B}_{n}}\left(\sum_{i=1}^{\# V_{n-1}} m_{i} v_{i}^{n-1}\right)=\sum_{j=1}^{\# V_{n}}\left(\sum_{i=1}^{\# V_{n-1}} \#\left(s^{-1}\left\{v_{i}^{n-1}\right\} \cap r^{-1}\left\{v_{j}^{n}\right\}\right) m_{i}\right) v_{j}^{n} .
$$

Proposição 11.2.1. Para cada $n \geq 1$, a função $h_{\mathfrak{B}_{n}}$ é um homomorfismo positivo.

Prova. Fixe $n \geq 0$. Sejam

$$
g_{1}=\sum_{i=1}^{\# V_{n-1}} m_{i} v_{i}^{n-1} \quad \text { e } \quad g_{2}=\sum_{i=1}^{\# V_{n-1}} \ell_{i} v_{i}^{n-1}
$$


elementos de $\mathbb{Z} V_{n-1}$. Temos que

$$
\begin{aligned}
h_{\mathfrak{B}_{n}}\left(g_{1}+g_{2}\right) & =h_{n}\left(\sum_{i=1}^{\# V_{n-1}}\left(m_{i}+\ell_{i}\right) v_{i}^{n-1}\right) \\
& =\sum_{j=1}^{\# V_{n}}\left(\sum_{i=1}^{\# V_{n-1}} \#\left(s^{-1}\left\{v_{i}^{n-1}\right\} \cap r^{-1}\left\{v_{j}^{n}\right\}\right)\right)\left(m_{i}+\ell_{i}\right) v_{j}^{n} \\
& =\sum_{j=1}^{\# V_{n}}\left(\sum_{i=1}^{\# V_{n-1}} \#\left(s^{-1}\left\{v_{i}^{n-1}\right\} \cap r^{-1}\left\{v_{j}^{n}\right\}\right)\right) m_{i} v_{j}^{n} \\
& +\sum_{j=1}^{\# V_{n}}\left(\sum_{i=1}^{\# V_{n-1}} \#\left(s^{-1}\left\{v_{i}^{n-1}\right\} \cap r^{-1}\left\{v_{j}^{n}\right\}\right)\right) \ell_{i} v_{j}^{n} \\
& =h_{\mathfrak{B}_{n}}\left(g_{1}\right)+h_{\mathfrak{B}_{n}}\left(g_{2}\right) .
\end{aligned}
$$

Então, $h_{\mathfrak{B}_{n}}$ é homomorfismo. Além disso, como

$$
\#\left(s^{-1}\left\{v_{i}^{n-1}\right\} \cap r^{-1}\left\{v_{j}^{n}\right\}\right) \geq 0
$$

temos que $h_{\mathfrak{B}_{n}}\left(\mathbb{Z} V_{n-1}^{+}\right) \subset \mathbb{Z} V_{n}^{+}$. Portanto, $h_{\mathfrak{B}_{n}}$ é um homomorfismo positivo.

Como $n$ é arbitrário, concluímos a demonstração da proposição.

Dado $n \geq 0$, fixe $i$, tal que $1 \leq i \leq n$. Temos que $v_{i}^{n-1} \in V_{n-1} \subset \mathbb{Z} V_{n-1}$ e

$$
h_{\mathfrak{B}_{n}}\left(v_{i}^{n-1}\right)=\sum_{j=1}^{\# V_{n}} \#\left(s^{-1}\left\{v_{i}^{n-1}\right\} \cap r^{-1}\left\{v_{j}^{n}\right\}\right) v_{n, j} .
$$

Mas, \# $\left(s^{-1}\left\{v_{i}^{n-1}\right\} \cap r^{-1}\left\{v_{j}^{n}\right\}\right)>0$, se e somente se, $s^{-1}\left\{v_{i}^{n-1}\right\} \cap r^{-1}\left\{v_{j}^{n}\right\} \neq \emptyset$. Isto é, se existe $e \in E_{n}$, tal que $s(e)=v_{i}^{n-1}$ e $r(e)=v_{j}^{n}$. Então, podemos reescrever $h_{\mathfrak{B}_{n}}\left(v_{i}^{n-1}\right)$ como:

$$
h_{\mathfrak{B}_{n}}\left(v_{i}^{n-1}\right)=\sum_{e \in E_{n}, s(e)=v_{i}^{n-1}} r(e)
$$

Teorema 11.2.2. Seja $V=\left\{V_{n}\right\}_{n \geq 0}$ uma coleção de conjuntos finitos. Se para todo $n \geq 1, h_{n}: \mathbb{Z} V_{n-1} \rightarrow \mathbb{Z} V_{n}$ é um homomorfismo positivo então existe um diagrama de Bratteli $\mathfrak{B}=(V, E, r, s)$, onde $V=\left\{V_{n}\right\}_{n \geq 0}$ e $h_{\mathfrak{B}_{n}}=h_{n}$.

Prova. Definiremos para cada $n \geq 1$, os conjuntos $E_{n}$. Seja $v_{i}^{n-1} \in V_{n-1} \subset$ $\mathbb{Z} V_{n-1}^{+}$, então $h_{n}\left(v_{i}^{n-1}\right)=\sum_{j=1}^{\# V_{n}} m_{i, j} v_{j}^{n}$. Como $h_{n}$ é positivo, temos que $m_{i, j} \geq 0$ para todo $1 \leq j \leq \# V_{n}$. Definimos que existem $m_{i, j}$ arestas ligando o vértice $v_{i}^{n-1}$ ao vértice $v_{j}^{n}$, que denotamos por $e_{i, j, \ell}$, onde $1 \leq \ell \leq m_{i, j}$. Desta forma, para cada $n \geq 0$

$$
E_{n}:=\left\{e_{i, j \cdot \ell} \mid 1 \leq i \leq \# V_{n-1}, 1 \leq j \leq \# V_{n}, 1 \leq \ell \leq m_{i, j}\right\}
$$


Para cada $e_{i, j, l} \in E_{n}$, definimos

$$
s\left(e_{i, j, \ell}\right)=v_{i}^{n-1} \quad \text { e } \quad r\left(e_{i, j, \ell}\right)=v_{j}^{n} .
$$

Como $m_{i, j} \geq 0, s^{-1}(v), r^{-1}(v) \neq \emptyset$ para todo $v \in\left\{V_{n}\right\}_{n \geq 0}$. Então, a coleção

$$
\mathfrak{B}=\left(V=\left\{V_{n}\right\}_{n \geq 0}, E=\left\{E_{n}\right\}_{n \geq 1}, r, s\right)
$$

é um diagrama de Bratteli.

Mostraremos que $h_{\mathfrak{B}_{n}}=h_{n}$. Seja $g=\sum_{i=1}^{\# V_{n-1}} k_{i} v_{i}^{n-1}$, usando que $h_{n}$ é homomorfismo, temos que

$$
\begin{aligned}
h_{n}(g) & =h_{n}\left(\sum_{i=1}^{\# V_{n-1}} k_{i} v_{i}^{n-1}\right) \\
& =\sum_{i=1}^{\# V_{n}} k_{i} h_{n}\left(v_{i}^{n-1}\right)=\sum_{i=1}^{\# V_{n-1}} k_{i}\left(\sum_{j=1}^{\# V_{n}} m_{i, j} v_{j}^{n}\right) .
\end{aligned}
$$

Mas, por definição

$$
m_{i, j}=\#\left(s^{-1}\left\{v_{i}^{n-1}\right\} \cap r^{-1}\left\{v_{j}^{n}\right\}\right)
$$

Então $h_{n}(g)=h_{\mathfrak{B}_{n}}(g)$. Como $g$ é arbitrário, temos que $h_{\mathfrak{B}_{n}}=h_{n}$. Concluímos, assim, a prova do teorema.

Dado um diagrama de Bratteli $\mathfrak{B}=(V, E, r, s)$, considere $m$ e $n$ números naturais tais que $m>n$. Considere a função $h_{\mathfrak{B}_{n, m}}: \mathbb{Z} V_{n} \rightarrow \mathbb{Z} V_{m}$, definida por:

$$
h_{\mathfrak{B}_{n, m}}=h_{\mathfrak{B}_{m}} \circ h_{\mathfrak{B}_{m-2}} \circ \cdots \circ h_{\mathfrak{B}_{n+1}} .
$$

Observação 11.2.3. Seja $k$ tal que $n<k<m$, temos que

$$
h_{\mathfrak{B}_{k, m}} \circ h_{\mathfrak{B}_{n, k}}=h_{\mathfrak{B}_{m, n}} .
$$

Observação 11.2.4. Dado $n \geq 1, h_{\mathfrak{B}_{n, n+1}}=h_{\mathfrak{B}_{n+1}}$.

Dado $n \geq 1$, se o conjunto $V_{n}$ possui $k_{n}$ elementos, então o conjunto $\mathbb{Z} V_{n}$ é isomorfo a $\mathbb{Z}^{k_{n}}$. Assim, os elementos de $\mathbb{Z}^{k_{n}}$ podem ser vistos como vetores coluna e o homomorfismo $h_{\mathfrak{B}_{n}}$ como uma matriz $k_{n} \times k_{n-1}$, que será denotada por $\left(H_{\mathfrak{B}_{n}}\right)=\left(h_{\mathfrak{B}_{n}, i, j}\right)$, onde $\left(h_{\mathfrak{B}_{n}, i, j}\right)$ é o número de arestas em $E_{n}$ que ligam $v_{i}^{n-1}$ a $v_{j}^{n}$. 


\subsection{2}

\section{O grupo de dimensão de um diagrama de Bratteli}

Finalmente estamos prontos para definir o grupo de dimensão de um diagrama de Bratteli. Para isto, lembre que dada uma sequência $\left\{G_{n}\right\}_{n \geq 0}$ de grupos abelianos e uma sequência $\left\{\alpha_{n}\right\}_{n \geq 1}$ de homomorfismos positivos, definimos o grupo $\left(\mathbf{G}_{\infty}, \alpha_{\infty}\right)$.

Definição 11.2.5 (Grupo de dimensão de um diagrama de Bratteli). Seja $\mathfrak{B}=(V, E, r, s)$ um diagrama de Bratteli. Definimos seu grupo de dimensão como o conjunto

$$
D(\mathfrak{B})=\left(\mathbb{Z} V_{\infty}, h_{\infty}\right)
$$

Repare que o Teorema 11.1.9 nos dá um cone positivo para o grupo $D(\mathfrak{B})$. Então o grupo $D(\mathfrak{B})$ é um grupo abeliano ordenado.

Estudaremos o seguinte exemplo.

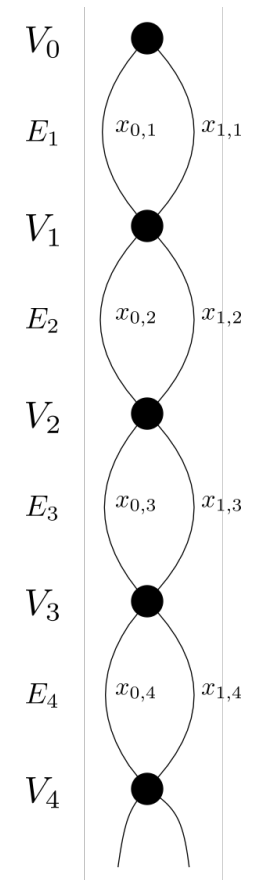

Figura 11.1: Diagrama de Bratteli $\mathfrak{B}$

Considere o diagrama de Bratteli $\mathfrak{B}=(V, E, r, s)$ tal que $\left(X_{\mathfrak{B}}, \varphi_{\mathfrak{B}}\right)$ é conjugado com $\left(\{0,1\}^{\mathbb{N}}, \Phi\right)$, onde $\Phi$ é o odômetro diádico, apresentado na Figura 11.1. Identificaremos o seu grupo de dimensão $D(\mathfrak{B})$. Como para cada $n \geq 0, V_{n}=\left\{v_{n}\right\}$ e $\# E_{n}=2$, temos que

$$
h_{\mathfrak{B}_{n-1}}\left(k v_{n}\right)=k 2 v_{n+1}, \quad k \in \mathbb{Z} .
$$


Observe que a função $h_{\mathfrak{B}_{n}}$ é injetiva, para todo $n \geq 0$. Considere o elemento $\left\langle v_{0}, 0\right\rangle$ de $\left(\mathbb{Z} V_{\infty}, h_{\infty}\right)$, temos que

$$
\begin{aligned}
\left\langle v_{0}, 0\right\rangle & =\left\langle h_{\mathfrak{B}_{1}}\left(v_{0}\right), 1\right\rangle=\left\langle 2 v_{1}, 1\right\rangle=2\left\langle v_{1}, 1\right\rangle=2\left\langle h_{\mathfrak{B}_{2}}\left(v_{1}\right), 2\right\rangle \\
& =4\left\langle v_{2}, 2\right\rangle=\cdots=2^{k}\left\langle v_{k}, k\right\rangle .
\end{aligned}
$$

Definimos o grupo

$$
\mathbb{Z}[1 / 2]:=\left\{\frac{p}{2^{n}} \mid p \in \mathbb{Z}, n \in \mathbb{N}\right\},
$$

que chamaremos de grupo diádico. Definimos, também, a função

$$
\beta: D(\mathfrak{B}) \rightarrow\left\{\frac{p}{2^{n}} \mid p \in \mathbb{Z}, n \in \mathbb{N}\right\}, \quad \beta\left(\left\langle p v_{n}, n\right\rangle\right)=\frac{p}{2^{n}}
$$

Proposição 11.2.6. A função $\beta$ é um isomorfismo.

Prova. Considere dois elementos $\left\langle p v_{n}, n\right\rangle$ e $\left\langle q v_{k}, k\right\rangle$ de $D(\mathfrak{B})$. Mostraremos, primeiro, a injetividade. Suponha que $\beta\left(\left\langle p v_{n}, n\right\rangle\right)=\beta\left(\left\langle q v_{k}, k\right\rangle\right)$ e que $k \leq n$, segue que

$$
\frac{p}{2^{n}}=\frac{q}{2^{k}} \Leftrightarrow p=2^{n-k} q
$$

Então $\left\langle p v_{n}, n\right\rangle=\left\langle 2^{n-k} q v_{n}, n\right\rangle$. Além disso, temos que

$$
h_{\mathfrak{B}_{n}}^{k}\left(p v_{n}\right)=h_{\mathfrak{B}_{n}}^{k}\left(2^{n-k} q v_{n}\right)=2^{k} 2^{n-k} q v_{n+k}=2^{n} q v_{n+k}=h_{\mathfrak{B}}^{n}\left(q v_{k}\right) .
$$

Portanto, $\left(p v_{n}, n\right) \sim\left(q v_{k}, k\right)$ e $\left\langle p v_{n}, n\right\rangle=\left\langle q v_{k}, k\right\rangle$. Assim, $\beta$ é uma função injetiva. Por construção $\beta$ é uma função sobrejetiva.

Só falta mostrar que $\beta$ é um homomorfismo. Sejam $\left\langle p v_{n}, n\right\rangle$ e $\left\langle q v_{k}, k\right\rangle$ dois elementos em $R_{\mathfrak{B}}$. Suponha que $k \leq n$, temos que

$$
\left\langle q v_{k}, k\right\rangle=\left\langle 2^{n-k} q v_{n}, n\right\rangle
$$

Então

$$
\begin{aligned}
\beta\left(\left\langle p v_{n}, n\right\rangle+\left\langle q v_{k}, k\right\rangle\right) & =\beta\left(\left\langle\left(p+2^{n-k} q\right) v_{n}, n\right\rangle\right) \\
& =\frac{p+2^{n-k} q}{2^{n}}=\frac{p}{2^{n}}+\frac{q}{2^{k}} \\
& =\beta\left(\left\langle p v_{n}, n\right\rangle\right)+\beta\left(\left\langle q v_{n}, n\right\rangle\right) .
\end{aligned}
$$

Concluímos, assim que $\beta$ é um homomorfismo e portanto um isomorfismo.

A seguir identificaremos o grupo de dimensão do diagrama de Bratteli $\mathfrak{T}=(W, F, r, s)$ na Figura 11.2. 


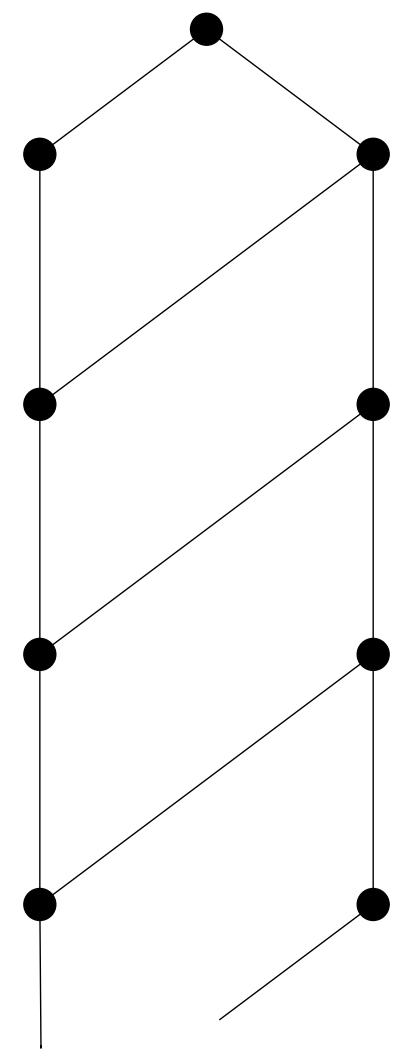

Figura 11.2: Diagrama de Bratteli $\mathfrak{T}=(W, F, r, s)$

Denotamos

$$
W_{0}=\left\{w_{0}^{0}\right\}
$$

e para $n \geq 1$,

$$
W_{n}=\left\{w_{i}^{n} \mid 1 \leq i \leq 2\right\}
$$

Agora, denotamos

$$
E_{1}=\left\{f_{(0,1)^{1}}, f_{(0,2)^{1}}\right\},
$$

e para $n \geq 2$,

$$
E_{n}=\left\{f_{(1,1)^{n}}, f_{(1,2)^{n}}, f_{(2,2)^{n}}\right\} .
$$

Lembre que usando a caracterização por meio de matrizes, podemos representar o homomorfismo $h_{\mathfrak{T}_{n}}$ por uma matriz $H_{\mathfrak{T}_{n}}=\left(h_{\mathfrak{T}_{n, i, j}}\right)$, onde $\left(h_{\mathfrak{T}_{n, i, j}}\right)$ é o número de arestas entre o vértice $v_{j}^{n-1}$ e o vértice $v_{j}^{n}$. Com o intuito de simplificar a notação, denotaremos, para cada $n \geq 0$ a matriz $H_{\mathfrak{T}_{n}}$ por $H_{n}$. Assim,

$$
H_{1}=\left[\begin{array}{l}
1 \\
1
\end{array}\right]
$$


e para todo $n \geq 2$,

$$
H_{n}=\left[\begin{array}{ll}
1 & 1 \\
0 & 1
\end{array}\right] .
$$

Como para todo $n \geq 2$, $\operatorname{det}\left(H_{n}\right)=1$, temos que para todo $n \geq 2$ a matriz $H_{n}$ é invertível e portanto, o homomorfismo $h_{\mathfrak{T}_{n}}$ é um isomorfismo. Considere, para todo $n \geq 0$, a função $\beta_{n}: \mathbb{Z} V_{n} \rightarrow D(\mathfrak{T})$ definida na equação (11.1.1). Como para todo $n \geq 1$, a função $h_{\mathfrak{T}_{n}}$ é um isomorfismo. Usando a condição 2 da Proposição 11.1.4 para $\alpha_{n}=h_{\mathfrak{T}_{n}}$ concluímos que

$$
\beta_{1}: \mathbb{Z} V_{1} \rightarrow D(\mathfrak{T})
$$

é um isomorfismo. Além disso, temos que $\mathbb{Z} V_{1} \cong \mathbb{Z}^{2}$. Concluímos, portanto, que $D(\mathfrak{T}) \cong \mathbb{Z}^{2}$.

Seria intuitivo pensar que o cone positivo de $D(\mathfrak{T})$ é

$$
D(\mathfrak{T})^{+}=\{(i, j) \mid i, j \geq 0\}
$$

Mas não é o que acontece. Pelo Teorema 11.1.9, temos que

$$
D(\mathfrak{T})^{+}=\left\{(i, j) \mid\langle(i, j), 1\rangle=\langle a, n\rangle \text { para algum } n \geq 0 \text { e } a \in \mathbb{Z} V_{n}^{+}\right\} .
$$

Considere o elemento $(-1,1)$ de $D(\mathfrak{T})$. Temos que

$$
H_{2}\left(\left[\begin{array}{c}
-1 \\
1
\end{array}\right]\right)=\left[\begin{array}{l}
0 \\
1
\end{array}\right] .
$$

Portanto

$$
\langle(-1,1), 1\rangle=\left\langle h_{\mathfrak{T}_{2}}(-1,1), 2\right\rangle=\langle(0,1), 2\rangle .
$$

Então, $(-1,1)$ está em $D(\mathfrak{T})^{+}$.

Afirmação 11.2.7. $D(\mathfrak{T})^{+}=\{(i, j) \mid j>0$ ou $j=0$ e $i \geq 0\}$.

Prova. Sejam $i, j \in \mathbb{Z}$. Se $j=0$ e $k \geq 0$ é imediato que $(i, j) \in D(\mathfrak{T})^{\mathbb{N}}$.

Agora, considere que $j>0$ e $n>0$.

$$
H_{n}\left(\left[\begin{array}{l}
i \\
j
\end{array}\right]\right)=\left[\begin{array}{c}
i+j \\
j
\end{array}\right] .
$$

Então, existe $n$ tal que $i+n j>0$. Assim, $h_{\mathfrak{T}_{n}}(i, j) \in D(\mathfrak{T})^{+}$. Assim, $(i, j) \in D(\mathfrak{T})^{\mathbb{N}}$. 
Portanto,

$$
\{(i, j) \mid j>0 \text { ou } j=0 \text { e } i \geq 0\} \subset D(\mathfrak{T})^{+}
$$

Falta mostrar que

$$
D(\mathfrak{T})^{+} \subset\{(i, j) \mid j>0 \text { ou } j=0 \text { e } i \geq 0\}
$$

Dado $(i, j) \in D(\mathfrak{T})^{+}$, denotaremos $(i, j)$ por $\langle(i, j), 1\rangle$. Existem $n$ e $a \in \mathbb{Z} V_{n}^{+}$ tais que $\langle(i, j), 1\rangle=\langle a, n\rangle$. Como $a \in \mathbb{Z} V_{n}^{+}$, temos que

$$
\langle(i, j), 1\rangle=\langle p(1,0), n\rangle+q\langle(0,1), n\rangle, \quad \text { onde } p, q \geq 0 .
$$

Como $H_{n}$ é sempre a mesma matriz para todo $n \geq 2$, escreveremos $H$. Fixe $n \geq 2$, temos que

$$
H^{1-n}=\left[\begin{array}{cc}
1 & 1-n \\
0 & 1
\end{array}\right] \text {. }
$$

Então,

$$
H^{1-n}(1,0)=(1,0) \quad \text { e } \quad H^{1-n}(0,1)=(1-n, 1) .
$$

Portanto

$$
\langle(1,0), n\rangle=\left\langle H^{1-n}(1,0), 1\right\rangle=\langle(1,0), 1\rangle
$$

e

$$
\langle(0,1), n\rangle=\left\langle H^{1-n}(0,1), 1\right\rangle=\langle(1-n, 1), 1\rangle .
$$

Substituindo na equação (11.2.2), obtemos

$$
\langle(i, j), 1\rangle=p\langle(1,0), 1\rangle+q\langle(1-n, 1), 1\rangle
$$

. Como $n \geq 2$, temos que $1-n \leq 0$, então

$$
\langle(i, j), 1\rangle \in\{(i, j) \mid j>0 \text { ou } j=0 \text { e } i \geq 0\} .
$$

Concluímos, assim, que

$$
D\left(\mathfrak{T}^{+}\right)=\{(i, j) \mid j>0 \text { ou } j=0 \text { e } i \geq 0\} .
$$

Finalizamos, então, a demonstração da afirmação. 


\subsection{3}

\section{O grupo de dimensão e a propriedade telescópica}

Agora que já vimos alguns exemplos sobre o grupo de dimensão, pode surgir a pergunta: o que acontece com o grupo de dimensão de um diagrama de Bratteli se aplicarmos a propriedade telescópica a este?

Seja $\mathfrak{B}=(V, E, r, s)$ um diagrama de Bratteli. Considere uma sequência crescente de inteiros $\left\{m_{k}\right\}_{k \geq 0}$ tal que $m_{0}=0$ e o diagrama $\mathfrak{T}=(W, F, \bar{r}, \bar{s})$ obtido a partir de $\mathfrak{B}$ através da subsequência $\left\{m_{k}\right\}_{k \geq 0}$. Considere a função $t: D(\mathfrak{T}) \rightarrow D(\mathfrak{B})$, definida por

$$
t(\langle w, k\rangle)=\left\langle w, m_{k}\right\rangle
$$

Teorema 11.2.8. A função t é um isomorfismo.

Prova. Claramente a função é um homomorfismo. Precisamos mostrar que é uma bijeção. Veremos primeiro, que a função é sobrejetiva. Seja $\langle v, n\rangle$ um elemento de $D(\mathfrak{B})$. Lembre da função $h_{\mathfrak{B}_{n}}: \mathbb{Z} V_{n-1} \rightarrow \mathbb{Z} V_{n}$, definida por:

$$
h_{\mathfrak{B}_{n}}\left(\sum_{i=1}^{\# V_{n}} m_{i} v_{i}^{n-1}\right)=\sum_{j=1}^{\# V_{n}}\left(\sum_{i=1}^{\# V_{n-1}} \#\left(s^{-1}\left\{v_{i}^{n-1} \cap r^{-1}\left\{v_{j}^{n}\right\}\right) m_{i}\right) v_{j}^{n} .\right.
$$

Lembre também que dado $n<m$, definimos

$$
h_{\mathfrak{B}_{n, m}}=h_{\mathfrak{B}_{m}} \circ h_{\mathfrak{B}_{m-1}} \circ \cdots \circ h_{\mathfrak{B}_{n+1}} .
$$

Como para cada vértice $v$, os conjuntos $r^{-1}\{v\}$ e $s^{-1}\{v\}$ são não vazios, temos que para cada $k \geq 0$ existe $a \in \mathbb{Z} V_{m_{k}}$ tal que $\langle v, n\rangle=\left\langle a, m_{k}\right\rangle$. Então,

$$
\langle v, n\rangle=\left\langle a, m_{k}\right\rangle=t(\langle a, k\rangle)
$$

Portanto, $t$ é sobrejetiva.

Mostraremos, agora, que $t$ é injetiva. Sejam $\langle v, k\rangle$ e $\langle w, l\rangle$ em $D(\mathfrak{T})$. Suponha que

$$
t(\langle v, k\rangle)=t(\langle w, l\rangle)
$$

então

$$
\left\langle v, m_{k}\right\rangle=\left\langle w, m_{l}\right\rangle
$$

Portanto, existe inteiro $N>m_{k}, m_{l}$ tal que

$$
h_{\mathfrak{B}_{m_{k}, N}}(v)=h_{\mathfrak{B}_{m_{l}, N}}(w) .
$$

Podemos considerar somente o caso em que $k<l$. Temos que existe $w_{k}$ tal que 
$w=h_{\mathfrak{B}_{m_{k}, m_{l}}}\left(w_{k}\right)$. Então

$$
h_{\mathfrak{B}_{m_{k}, N}}(v)=h_{\mathfrak{B}_{m_{l}, N}}(w)=h_{\mathfrak{B}_{m_{l}, N}}\left(h_{\mathfrak{B}_{m_{k}, m_{l}}}\left(w_{k}\right)\right)=h_{\mathfrak{B}_{m_{k}, N}}\left(w_{k}\right)
$$

Como $h_{\mathfrak{B}_{i}}$ é injetiva para todo $i$, temos que $v=w_{k}, \operatorname{logo}\langle v, k\rangle=\left\langle w_{k}, k\right\rangle$. Além disso, como $w=h_{\mathfrak{B}_{m_{k}}, m_{l}}\left(w_{k}\right)$, temos que $\left\langle w_{k}, k\right\rangle=\langle w, l\rangle$. Portanto,

$$
\langle v, k\rangle=\langle w, l\rangle
$$

Então $t$ é injetiva. Concluímos, assim que a função $t$ é um isomorfismo.

\subsection{4}

\section{O grupo de dimensão e a ordem unitária}

A seguir, veremos alguns resultados sobre a ordem unitária em um grupo de dimensão. Lembre na Definição 9.2.1 o conceito de ordem unitária.

Observação 11.2.9. Seja $\mathfrak{B}=(V, E, r, s)$ um diagrama de Bratteli. Se $V_{0}=\left\{v_{0}\right\}$ então $\left\langle v_{0}, 0\right\rangle$ é uma ordem unitária para o seu grupo de dimensão $D(\mathfrak{B})$.

De fato, seja $\langle v, n\rangle$ um elemento de $D(\mathfrak{B})$, como $V_{0}$ possui somente o elemento $v_{0}$, existe $k \in \mathbb{Z}$ tal que $\langle v, n\rangle=\left\langle k v_{0}, 0\right\rangle$. Temos que

$$
k\left\langle v_{0}, 0\right\rangle-\langle v, n\rangle=k\left\langle v_{0}, 0\right\rangle-\left\langle k v_{0}, 0\right\rangle=\left\langle k v_{0}-k v_{0}, 0\right\rangle=0 \in D(\mathfrak{B})^{+} .
$$

Portanto, $\left\langle v_{0}, 0\right\rangle$ é uma ordem unitária para $D(\mathfrak{B})$.

Quando $V_{0}$ possui mais de um elemento, não podemos garantir que $V_{0}$ contenha algum elemento que seja ordem ordem unitária. Veja o exemplo: No diagrama de Bratteli apresentado na Figura 11.3, $\langle v, 0\rangle$ não é uma ordem unitária para todo $v \in V_{0}$.

Lema 11.2.10. Considere um diagrama de Bratteli $\mathfrak{B}=(V, E, r, s)$ e seu grupo de dimensão $D(\mathfrak{B})$. Se $\langle v, n\rangle$ é uma ordem unitária de $D(\mathfrak{B})^{+}$então dado $\langle w, m\rangle$ em $D(\mathfrak{B})$, existem inteiros $l$ e $N$, tais que

$$
h_{n, N}(l v) \geq h_{m, N}(w) .
$$

Prova. Considere $\langle v, n\rangle$ como uma ordem unitária de $D(\mathfrak{B})^{+}$. Por definição de ordem unitária, dado $\langle w, m\rangle$ em $D(\mathfrak{B})$ existe inteiro $\ell$ tal que

$$
\ell\langle v, n\rangle-\langle w, m\rangle \in D(\mathfrak{B})^{+}
$$




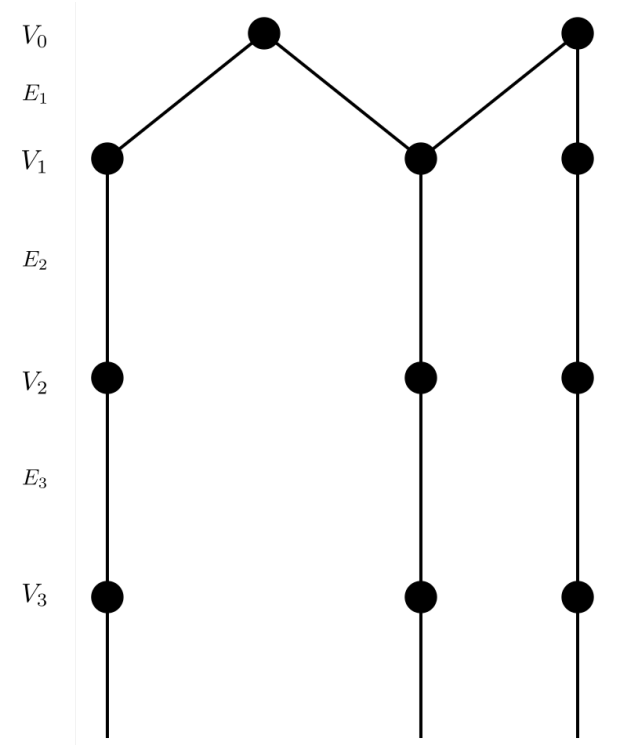

Figura 11.3: Exemplo de diagrama de Bratteli com mais de um elemento em $V_{0}$.

Sabemos que

$$
\langle w, m\rangle=\left\langle h_{m, n}(w), n\right\rangle .
$$

Podemos, então, reescrever a equação (11.2.3) como

$$
\ell\langle v, n\rangle-\left\langle h_{m, n}(w), n\right\rangle=\left\langle\ell v-h_{m, n}(w), n\right\rangle \in D(\mathfrak{B})^{+}
$$

Isso significa que existem inteiro $k$ e $a \in \mathbb{Z} V_{k}^{+}$tal que

$$
\left\langle v-h_{m, n}(w), n\right\rangle=\langle a, k\rangle .
$$

Então existem inteiro $N$, tal que

$$
h_{n, N}\left(\ell v-h_{m, n}(w)\right)=h_{k, N}(a) .
$$

Como $h_{i}$ é positivo para todo $i$, temos que

$$
\begin{aligned}
h_{n, N}\left(\ell v-h_{m, n}(w)\right) & =h_{n, N}(\ell v)-h_{n, N}\left(h_{m, n}(w)\right) \\
& =h_{n, N}(\ell v)-h_{m, N}(w) \in \mathbb{Z} V_{N}^{+} .
\end{aligned}
$$

Então,

$$
h_{n, N}(\ell v) \geq h_{m, N}(w) .
$$

Concluímos, assim, a demonstração do lema.

Teorema 11.2.11. Sejam $\mathfrak{B}=(V, E, r, s)$ um diagrama de Bratteli e $v_{0} \in V_{0}$ 
tal que $\left\langle v_{0}, 0\right\rangle$ é uma ordem unitária para $D(\mathfrak{B})$. Então existem um sub diagrama $\mathfrak{T}=(W, F, \bar{r}, \bar{s})$ tal que $W_{0}=\left\{v_{0}\right\}$ e um isomorfismo ordenado $\alpha$ entre $D(\mathfrak{T})$ e $D(\mathfrak{B})$ tal que $\alpha\left(\left\langle v_{0}, 0\right\rangle\right)=\left\langle v_{0}, 0\right\rangle$.

Prova. Temos que definir os conjuntos $W$ e $F$. Começaremos pelo conjunto $W$. Definimos $W_{0}=\left\{v_{0}\right\}$ e para todo $n \geq 1$,

$$
W_{n}:=\left\{w \in V_{n} \mid \text { existe caminho de } v_{0} \text { para } w\right\} \subset V_{n}
$$

O conjunto $W$ é a coleção $\left\{W_{n}\right\}_{n \geq 0}$

Agora, definimos

$$
F_{n}:=s_{n}^{-1}\left(W_{n-1}\right) \quad \text { e } \quad F=\left\{F_{n}\right\}_{n \geq 1} .
$$

Lembre que, para cada $n \geq 1, s_{n}: E_{N} \rightarrow V_{n-1}$, dessa forma, o conjunto $F$ é formado por todas as arestas que saem dos vértices que estão em $W_{n}$. Assim, Se está em $F$, existem $w$ em $W$ tal que $s(e)=w$ e caminho $p$ de $v_{0}$ a $w$. Dessa forma, a concatenação pe é um caminho de $v_{0}$ a $r(e)$ e, portanto, $r(e)$ está em $W$.

Por último, definimos para cada $n \geq 0$

$$
\bar{r}:=r_{\mid F} \quad \text { e } \quad \bar{s}:=s_{\mid F} .
$$

O diagrama de Bratteli $\mathfrak{T}=(W, F, \bar{r}, \bar{s})$ é um sub diagrama de $\mathfrak{B}$ tal que $W_{0}=\left\{v_{0}\right\}$.

Agora, seja a função definida por:

$$
\alpha: D(\mathfrak{T}) \rightarrow D(\mathfrak{B}), \quad \alpha\left(\langle w, n\rangle_{\mathfrak{T}}\right)=\langle w, n\rangle_{\mathfrak{B}}
$$

Lema 11.2.12. A função $\alpha$ é um isomorfismo ordenado positivo.

Prova. A função $\alpha$ claramente é um homomorfismo. Mostraremos que a função é bijetiva. Também é claro que a função é injetiva. Falta mostrar que a função é sobrejetiva. Dado $v \in V_{n}$, encontraremos $m>n$ e $w \in \mathbb{Z} W_{m}$ tal que

$$
\langle v, n\rangle_{\mathfrak{B}}=\langle w, m\rangle_{\mathfrak{B}}
$$

Usando o Lema 11.2.10, temos que existem inteiros $\ell>0$ e $m>n$ tais que

$$
h_{\mathfrak{B}_{0, m}}\left(\ell v_{0}\right) \geq h_{\mathfrak{B}_{n, m}}(v) .
$$

Podemos escrever, 


$$
h_{\mathfrak{B}_{0, m}}\left(\ell v_{0}\right)=\sum_{v_{m} \in V_{m}} \ell i_{v_{m}} v_{m} \quad \text { e } \quad h_{\mathfrak{B}_{n, m}}(v)=\sum_{v_{m} \in V_{m}} j_{v_{m}} v_{m}
$$

Pela definição da função $h_{\mathfrak{B}_{m, n}}, i_{v_{m}} \geq 0$ para todo $v_{m} \in V_{m}$. Como, $s^{-1}\{v\}$ e $r^{-1}\{v\}$ são não vazios para todo $v$, existe pelo menos um $v_{m} \in V_{m}$ tal que $j_{v_{m}}>0$. Usando as equações (11.2.5) e (11.2.6), temos que

$$
\ell i_{v_{m}} \geq j_{v_{m}}
$$

Como $\ell>0$ e $j_{v_{m}}>0$, obtemos que $i_{v_{m}}>0$. Isto significa que $v_{m} \in W_{m}$. Portanto, $h_{\mathfrak{B}_{n, m}}(v) \in \mathbb{Z} W_{m}$. Além disso,

$$
\langle v, v\rangle_{\mathfrak{B}}=\left\langle h_{\mathfrak{B}_{n, m}}(v), m\right\rangle_{\mathfrak{B}}
$$

Definimos,

$$
w=h_{\mathfrak{B}_{n, m}}(v)
$$

Temos que

$$
\alpha\left(\langle w, m\rangle_{\mathfrak{T}}\right)=\langle v, n\rangle .
$$

Portanto, $\alpha$ é uma função sobrejetiva. Note que $w \in \mathbb{Z} W_{m}^{+}$. Então $\alpha$ é um isomorfismo positivo. Assim, concluímos a prova do Teorema 11.2.11.

Lembre que um diagrama de Bratteli é simples se satisfaz alguma uma das condições (equivalentes) do Teorema 5.3.5. Podemos usar o grupo de dimensão para identificar quando um diagrama de Bratteli é simples.

Teorema 11.2.13. Um diagrama de Bratteli $\mathfrak{B}=(V, E, r, s)$ tal que $V_{0}=\left\{v_{0}\right\}$ é simples se e somente se todo elemento estritamente positivo de $D(\mathfrak{B})$ é uma ordem unitária.

Prova. Suponha que todo elemento estritamente positivo de $D((\mathfrak{B})$ seja uma ordem unitária. Dado $v \in V_{m}$ temos que $\langle v, m\rangle$ é uma ordem unitária. Portanto, pelo Lema 11.2.10 dado $v_{0} \in V_{0}$, existem inteiros positivos $\ell>0$ e $n>m$ tais que

$$
\ell h_{m, n}(v) \geq h_{0, n}\left(v_{0}\right)
$$

Temos que

$$
h_{m, n}(v)=\sum_{v_{n} \in V_{n}} i_{v_{n}} v_{n} \quad \text { e } \quad h_{0, n}\left(v_{0}\right)=\sum_{v_{n} \in V_{n}} j_{v_{n}} v_{n}
$$

Podemos escrever

$$
\ell\left(\sum_{v_{n} \in v_{n}} i_{v_{n}} v_{n}\right) \geq \sum_{v_{n} \in V_{n}} j_{v_{n}} v_{n}
$$


Como $s^{-1}(w) \neq \emptyset$ para todo $w \in V$, temos que $j_{v_{n}}>0$ para todo $v_{n}$ em $V_{n}$. Portanto, também temos que $i_{v_{n}}>0$ para todo $v_{n}$ em $V_{n}$. Isso significa que existe pelo menos um caminho entre $v$ e todo elemento de $V_{n}$. Isto é, o diagrama de Bratteli $\mathfrak{B}$ é simples.

Agora, suponha que o diagrama $\mathfrak{B}$ é simples. Seja $\langle v, m\rangle$ um elemento estritamente positivo de $D(\mathfrak{B})$. Mostraremos que $\langle v, m\rangle$ é uma ordem unitária. Como $\mathfrak{B}$ é simples, pelo item 1 do Teorema 5.3.5, existe $n(m)>m$ tal que existe um caminho entre $v$ e todo elemento de $V_{m}$. Dessa forma,

$$
h_{\mathfrak{B}_{m, n(m)}}(v)=\sum_{v_{n} \in V_{n(m)}} i_{v_{n}} v_{n}, \quad i_{v_{n}}>0 \text { para todo } v_{n} \in V_{n(m)} .
$$

Considere o elemento $\langle w, k\rangle$. Não há problema considerar, $k<m$, quando $k \geq m$, encontramos um representante de $\langle v, m\rangle$ em $V_{k}$ e tomamos $n(k)$. Temos que

$$
h_{\mathfrak{B}_{k, n}}(w)=\sum_{v_{n} \in v_{n}} j_{v_{n}} v_{n}, \quad j_{v_{n}} \geq 0 .
$$

Como $i v_{n}>0$ para todo $v_{n} \in V_{n}$, existe inteiro positivo $L$ tal que

$$
L i_{v_{n}} \geq j_{v_{n}}, \quad \text { para todo } v_{n} \in V_{n}
$$

Assim,

$$
L h_{\mathfrak{B}_{m, n}}(v) \geq h_{\mathfrak{B}_{k, n}}(w) \Leftrightarrow L\langle v, m\rangle \geq\langle w, k\rangle \Leftrightarrow L\langle v, m\rangle-\langle w, k\rangle \in D(\mathfrak{B})^{+} .
$$

Concluímos, assim, que o elemento $\langle v, m\rangle$ é uma ordem unitária. 


\section{O invariante para as relações AF e orbital}

O objetivo deste capítulo é dados um espaço métrico compacto $X$, um homeomorfismo minimal $\varphi: X \rightarrow X$ sem pontos periódicos e um diagrama de Bratteli $\mathfrak{B}$ encontrar os invariantes $D\left(X, R_{\varphi}\right)$ e $D\left(X_{\mathfrak{B}}, R_{\mathfrak{B}}\right)$.

\section{1}

\section{$\mathrm{O}$ invariante para relações-AF}

Lembre que relacionada a uma ação local $\Gamma$ há uma relação de equivalência $R_{\Gamma}$, definida na Seção 8.1, que é denominada de relação de equivalência étale.

$\mathrm{Na}$ Seção 8.2, vimos que a um diagrama de Bratteli $\mathfrak{B}=(V, E, r, s)$ pode ser associado a uma ação local $\Gamma_{\mathfrak{B}}$. No Teorema 8.2.3, demonstramos que $R_{\mathfrak{B}}=R_{\Gamma_{\mathfrak{B}}}$.

Recorde que uma uma relação de equivalência $R$ no espaço $X$ é $A F$ se existe um diagrama de Bratteli $\mathfrak{B}$ tal que $(X, R)$ é isomorfo a $\left(X_{\mathfrak{B}}, R_{\mathfrak{B}}\right)$.

Na Seção 10.1, definimos o invariante $D(X, R)$ para relações de equivalência étale. O objetivo desta seção é, dado um diagrama de Bratteli $\mathfrak{B}=(V, E, r, s)$, explorar a relação entre os invariantes $D\left(X_{\mathfrak{B}}, R_{\mathfrak{B}}\right)$ e $D(\mathfrak{B})$.

Teorema 12.1.1. Seja $\mathfrak{B}=(V, E, r, s)$ um diagrama de Bratteli tal que $V=\left\{v_{0}\right\}$ e $\left(X_{\mathfrak{B}}, R_{\mathfrak{B}}\right)$ sua relação de equivalência $A F$. Então existe um isomorfismo $\alpha: D\left(X_{\mathfrak{B}}, R_{\mathfrak{B}}\right) \rightarrow D(\mathfrak{B})$, tal que

$$
\begin{gathered}
\alpha\left(D\left(X_{\mathfrak{B}}, R_{\mathfrak{B}}\right)^{+}\right)=D(\mathfrak{B})^{+}, \\
\alpha(\langle\mathbf{1}\rangle)=\left\langle v_{0}, 0\right\rangle .
\end{gathered}
$$

Prova. Para provar o lema necessitamos de alguns ingredientes prévios. Fixe $N \geq 1$. Dado um elemento $p \in E_{0, N}$, denotamos por $C(p)$ o cilindro centrado em $p$ e por $C\left(X_{\mathfrak{B}}, \mathbb{Z}\right)$ o grupo formado pelas funções contínuas de $X_{\mathfrak{B}}$ em $\mathbb{Z}$. Definimos o grupo $G_{N}$ como o grupo gerado pelas funções características $\mathcal{X}_{C(p)}$, onde $p \in E_{0, N}$.

Lema 12.1.2. Para todo $N \geq 0, G_{N} \subset G_{N+1} e$

$$
\bigcup_{N} G_{N}=C\left(X_{\mathfrak{B}}, \mathbb{Z}\right)
$$


Prova. Observe que, por definição, $G_{N}$ é o subgrupo de $C\left(X_{\mathfrak{B}}, \mathbb{Z}\right)$ formado pelas funções que são constante nos elementos da partição de $X_{\mathfrak{B}}$ dada por

$$
\left\{C(p) \mid p \in E_{0, N}\right\}
$$

Pelo item (4) da Proposição 5.1.5, podemos escrever

$$
C(p)=\bigcup_{e \in E_{N+1}, s(e)=r(p)} C(p e)
$$

então

$$
\mathcal{X}_{C(p)}=\mathcal{X}_{\cup_{e \in E_{N+1}, s(e)=r(p)} C(p e)}=\sum_{e \in E_{N+1}, s(e)=r(p)} \mathcal{X}_{C(p e)} .
$$

Como a equação (12.1.1) vale para todo $\mathcal{X}_{C(p)} \in G_{N}$ e temos que $\mathcal{X}_{C(p e)} \in G_{N+1}$ para todo $e \in E_{N}$ tal que $s(e)=r(p)$ obtemos que $G_{N} \subset G_{N+1}$. Além disso, pela Proposição 10.1.4, temos que

$$
\bigcup_{N} G_{N}=C\left(X_{\mathfrak{B}}, \mathbb{Z}\right)
$$

Demonstrando assim o lema.

Lembre que que definimos o grupo $B\left(X_{\mathfrak{B}}, R_{\mathfrak{B}}\right)$ como o grupo gerado pelas funções $\mathcal{X}_{\pi_{2}(\gamma)}-\mathcal{X}_{\pi_{1}(\gamma)}$, onde $\gamma=(U, \gamma(U))$ é um homeomorfismo parcial compacto e aberto definido em $X_{\mathfrak{B}}$ e as funções $\pi_{1}$ e $\pi_{2}$ são, respectivamente, as projeções na primeira e na segunda coordenada. Observe que $B\left(X_{\mathfrak{B}}, R_{\mathfrak{B}}\right) \subset$ $C\left(X_{\mathfrak{B}}, \mathbb{Z}\right)$.

Agora, definimos o grupo $H_{N}$ como o grupo gerado por

$$
\left\{\mathcal{X}_{C(p)}-\mathcal{X}_{C(q)} \mid p, q \in E_{0, N}, r(p)=r(q)\right\}
$$

Como $p, q \in E_{0, N}$, temos que $H_{n} \subset G_{N}$.

Lema 12.1.3. Para todo $N \geq 0, H_{N} \subset B\left(X_{\mathfrak{B}}, R_{\mathfrak{B}}\right), H_{N} \subset H_{N+1} e$

$$
\bigcup_{N} H_{N}=B\left(X_{\mathfrak{B}}, R_{\mathfrak{B}}\right)
$$

Prova. Lembre, que dados dois elementos $p$ e $q$ em $E_{0, N}$, definimos o conjunto

$$
\gamma(p, q)=\left\{(\mathbf{x}, \mathbf{y}) \in X_{\mathfrak{B}} \times X_{\mathfrak{B}} \mid \mathbf{x} \in C(p), \mathbf{y} \in C(q), x_{n}=y_{n}, \forall n>N\right\} .
$$

Temos que

$$
\pi_{1}(\gamma(p, q))=C(p) \quad \text { e } \quad \pi_{2}(\gamma(p, q))=C(q)
$$


Pela Proposição 8.2.2, temos que $\gamma(p, q)$ é um homeomorfismo parcial compacto e aberto. Então $\mathcal{X}_{C(p)}-\mathcal{X}_{C(q)}$ é um elemento de $B\left(X_{\mathfrak{B}}, R_{\mathfrak{B}}\right)$. Logo, pela definição de $H_{N}$, temos que $H_{N}$ está contido em $B\left(X_{\mathfrak{B}}, R_{\mathfrak{B}}\right)$. Da mesma forma que fizemos na equação (12.1.1), obtemos que

$$
\mathcal{X}_{C(p)}-\mathcal{X}_{C(q)}=\sum_{e \in E_{N+1}, s(e)=r(p)=r(q)} \mathcal{X}_{C(p e)}-\mathcal{X}_{C(q e)}
$$

obtendo que $H_{N} \subset H_{N+1}$. Finalmente, pelo Lema 10.2.1, segue que

$$
\bigcup_{N} H_{N}=B\left(X_{\mathfrak{B}}, R_{\mathfrak{B}}\right)
$$

Concluímos assim a demonstração do lema.

Por definição, temos que

$$
D\left(X_{\mathfrak{B}}, R_{\mathfrak{B}}\right)=C\left(X_{\mathfrak{B}}, \mathbb{Z}\right) / B\left(X_{\mathfrak{B}}, R_{\mathfrak{B}}\right)
$$

Então,

$$
\bigcup_{N} G_{N} / \bigcup_{N} H_{N} \cong D\left(X_{\mathfrak{B}}, R_{\mathfrak{B}} .\right)
$$

Lema 12.1.4. Para todo $N \geq 0, G_{N} / H_{N} \cong \mathbb{Z} V_{N}$.

Prova. Considere a função $\alpha_{N}: G_{N} \rightarrow \mathbb{Z} V_{N}$, definida por

$$
\alpha_{N}\left(\sum_{p \in E_{0, N}} a_{p} \mathcal{X}_{C(p)}\right)=\sum_{p} a_{p} r(p) .
$$

A seguir, veremos que $\alpha_{N}$ é sobrejetiva e que $\operatorname{Ker}\left(\alpha_{N}\right)=H_{N}$. Isto implica diretamente o lema.

Afirmação 12.1.5. A função $\alpha_{N}$ é sobrejetiva.

Prova. Considere $v=\sum_{v_{n} \in \mathbb{Z} V_{N}} a_{n} v_{n}$ um elemento de $\mathbb{Z} V_{N}$. Como $V_{0}=\left\{v_{0}\right\}$, para cada $v_{n}$ existe pelo menos um caminho de $v_{0}$ até $v_{n}$, que denotaremos por $p_{n}$. Temos que $r\left(p_{n}\right)=v_{n}$, dessa forma

$$
\alpha_{N}\left(\sum_{n} a_{n} \mathcal{X}_{C\left(p_{n}\right)}\right)=\sum_{n} a_{n} r\left(p_{n}\right)=\sum_{n} a_{n} v_{n}=v
$$

Portanto a função $\alpha_{N}$ é sobrejetiva, assim $\alpha_{N}\left(G_{N}\right)=\mathbb{Z} V_{N}$. Portanto, $\alpha_{N}$ é sobrejetiva.

Afirmação 12.1.6. Para todo $N \geq 0, \operatorname{Ker}\left(\alpha_{N}\right)=H_{N}$. 
Dados $p$ e $q$ em $E_{0 . N}$, tais que $r(p)=r(q)$ temos que

$$
\alpha_{N}\left(\mathcal{X}_{C(p)}-\mathcal{X}_{C(q)}\right)=r(p)-r(q)=0
$$

Então $H_{N} \subset \operatorname{Ker}\left(\alpha_{N}\right)$.

Agora, considere um elemento $g \in \operatorname{Ker}\left(\alpha_{N}\right)$, tal que $g \neq 0$. Podemos escrever,

$$
g=\sum_{i=1}^{k} b_{i} \mathcal{X}_{C\left(p_{i}\right)}, \quad b_{i}= \pm 1, \text { para todo } 1 \leq i \leq k .
$$

Onde os elementos $p_{i} \in E_{0, N}$ estão contados com multiplicidade. Temos que

$$
\alpha_{N}(g)=\sum_{i=1}^{k} b_{i} r\left(p_{i}\right)=0 .
$$

Como os elementos $p_{i}$ estão contados com multiplicidade, podemos escrever que

$$
\sum_{i=1}^{k} b_{i} r\left(p_{i}\right)=\sum_{j=1}^{\# V_{n}}\left(b_{j_{1}}+\cdots+b_{j_{l(j}}\right) v_{j},
$$

onde $l(j)=\#\left\{p_{i} \in E_{0, n} \mid r\left(p_{i}\right)=v_{j}\right\}$. Temos então, que

$$
=\sum_{j=1}^{\# V_{n}}\left(b_{j_{1}}+\cdots+b_{j_{l j}}\right) v_{j}=0 .
$$

Para isto ocorrer deve haver, dentre os coeficientes $b_{j_{1}}, \ldots, b_{j_{l(j)}}$, um número igual de coeficientes que são iguais a 1 e a -1 . Desta forma, $g \in H_{N}$. Concluímos que $\operatorname{Ker}\left(\alpha_{N}\right)=H_{N}$.

Pelo Teorema 9.1.4 existe um isomorfismo entre $G_{N} / \operatorname{Ker}\left(\alpha_{N}\right)$ e $\operatorname{Im}\left(\alpha_{N}\right)$. Então existe um isomorfismo entre $G_{N} / H_{N}$ e $\mathbb{Z} V_{N}$, que leva $a+H_{N}$ em $\alpha_{N}(a)$, que denotaremos por $f_{N}$.

Concluímos, assim, a demonstração do Lema 12.1.4.

Considere a função $\tilde{\alpha}_{N}: G_{N} / H_{n} \rightarrow G_{N+1} / H_{N+1}$ definida por

$$
\tilde{\alpha}_{N}\left(a+H_{N}\right)=a+H_{N+1} .
$$

Lembre que

$$
\bigcup_{N} G_{N} / \bigcup_{N} H_{n}=C\left(X_{\mathfrak{B}}, \mathbb{Z}\right) / B\left(X_{\mathfrak{B}}, R_{\mathfrak{B}}\right.
$$

Pela Proposição 11.1.7, existe um isomorfismo

$$
\Phi: C(X, \mathbb{Z}) / B\left(X_{\mathfrak{B}}, R_{\mathfrak{B}}\right) \rightarrow\left((G / H)_{\infty}, \tilde{\alpha}_{\infty}\right)
$$


tal que

$$
\Phi\left(a+B\left(X_{\mathfrak{B}}, R_{\mathfrak{B}}\right)=\left\langle a+H_{N}, N\right\rangle .\right.
$$

Em particular, se $p \in E_{0, N}$, temos que

$$
\Phi\left(\mathcal{X}_{C(p)}+B\left(X_{\mathfrak{B}}, R_{\mathfrak{B}}\right)\right)=\left\langle\mathcal{X}_{C(p)}+H_{N}, N\right\rangle .
$$

Mas, $\mathcal{X}_{C(p)}+H_{N} \in G_{N} / H_{N}$, então podemos levar $\left\langle\mathcal{X}_{C(p)}+H_{N}, N\right\rangle$ em

$$
\left\langle\alpha_{N}\left(\mathcal{X}_{C(p)}\right), N\right\rangle=\langle r(p), N\rangle .
$$

Então, temos um isomorfismo $\alpha: D\left(X_{\mathfrak{B}}, R_{\mathfrak{B}}\right) \rightarrow D(\mathfrak{B})$, que satisfaz

$$
\alpha\left(\mathcal{X}_{C(p)}\right)=\langle r(p), N\rangle \text {. }
$$

Escrevendo $\mathbf{1}=\sum_{e \in E_{1}} \mathcal{X}_{C(e)}$, temos que

$$
\begin{aligned}
\alpha(\langle\mathbf{1}\rangle)=\left(\sum_{e \in E_{1}} \mathcal{X}_{C}(e)+B\left(X_{\mathfrak{B}}, R_{\mathfrak{B}}\right)\right) & =\sum_{e \in E_{1}}\left\langle\alpha_{1}\left(\mathcal{X}_{C(e)}\right), 1\right\rangle \\
& =\sum_{e \in E_{1}}\langle r(e), 1\rangle \\
& =\left\langle\sum_{e \in E_{1}} r(e), 1\right\rangle \\
& =\left\langle h_{\mathfrak{B}_{1}}\left(v_{0}\right), 1\right\rangle \\
& =\left\langle v_{0}, 0\right\rangle .
\end{aligned}
$$

Agora só falta mostrar que

$$
\alpha\left(D\left(X_{\mathfrak{B}}, R_{\mathfrak{B}}\right)^{+}\right)=D(\mathfrak{B})^{+} .
$$

Seja $f \in D\left(X_{\mathfrak{B}}, R_{\mathfrak{B}}\right)^{+}$, então $f$ é uma função positiva em $C\left(X_{\mathfrak{B}}, \mathbb{Z}\right)$ e existe $N \geq 1$ tal que

$$
f=\sum_{p \in E_{0}, N} a_{p} \mathcal{X}_{C(p)}, \quad a_{p} \geq 0 .
$$

Portanto,

$$
\alpha(f)=\alpha\left(\sum_{p \in E_{0}, N} a_{p} \mathcal{X}_{C(p)}\right)=\left\langle\sum_{p \in E_{0, N}} a_{p} r(p), N\right\rangle=\sum_{p \in E_{0, N}} a_{p}\langle r(p), N\rangle .
$$

Como $a_{p} \geq 0$ para todo $p \in E_{0, N}, \alpha(f) \in D(\mathfrak{B})^{+}$e portanto

$$
\alpha\left(D\left(X_{\mathfrak{B}}, R_{\mathfrak{B}}\right)\right)^{+} \subset D(\mathfrak{B})^{+}
$$

Finalmente, considere um elemento de $D(\mathfrak{B})^{+}$. Podemos representá-lo da 
forma $\langle a, N\rangle$ par algum $N \geq 0$ e $a \in \mathbb{Z} V_{N}^{+}$. Então

$$
a=\sum_{v_{n} \in V_{N}} a_{n} v_{n}, \quad a_{n} \geq 0
$$

Para cada $v_{n} \in V_{N}$, existe caminho $p_{n}$ de $v_{0}$ até $v_{n}$, então

$$
\alpha\left(\sum_{n} a_{n} \mathcal{X}_{C\left(p_{n}\right)}\right)=\sum_{n} a_{n} r\left(p_{n}\right)=\sum_{v_{n} \in E_{0, N}} a_{n} v_{n}
$$

Como $a_{n} \geq 0$, o elemento $\sum_{n} a_{n} \mathcal{X}_{C\left(p_{n}\right)}$ está em $D\left(X_{\mathfrak{B}}, R_{\mathfrak{B}}\right)^{+}$e portanto

$$
D(\mathfrak{B})^{+} \subset \alpha\left(D\left(X_{\mathfrak{B}}, R_{\mathfrak{B}}\right)\right)
$$

Concluímos, assim, a demonstração do teorema.

\section{2}

\section{O invariante para o modelo de Bratteli-Vershik}

Lembre da definição de relação orbital associada a um homeomorfismo $\varphi$ na Definição 2.2.6.

Lembre que definimos no Capítulo 6 um diagrama de Bratteli ordenado $\mathfrak{B}=(V, E, r, s \geq)$ e a função de Bratteli-Vershik $\varphi_{\mathfrak{B}}: X_{\mathfrak{B}} \rightarrow X_{\mathfrak{B}}$ tal que $\varphi_{\mathfrak{B}}\left(\mathbf{x}_{\max }\right)=\mathbf{x}_{\min }$ e que leva um elemento $\mathbf{x}=\left(x_{n}\right)_{n} \neq \mathbf{x}_{\max }$ no elemento $\varphi_{\mathfrak{B}}(\mathbf{x})=\left(y_{1}, \ldots, y_{n}, x, \ldots\right)$, onde $y_{n}$ é tal que $r\left(x_{n}\right)=r\left(y_{n}\right)$ e é o elemento seguinte a $x_{n} \geq \mathrm{e}\left(y_{1}, \ldots, y_{n-1}\right)$ é o único caminho em $E_{\min }$ que liga $v_{0}$ a $s\left(y_{n}\right)$. Ao diagrama de Bratteli $\mathfrak{B}$ e a função $\varphi_{\mathfrak{B}}$ associamos a relação de equivalência $R_{\varphi_{\mathfrak{B}}}$. Neste capítulo determinaremos o grupo de dimensão associado ao espaço $\left(X_{\mathfrak{B}}, R_{\varphi_{\mathfrak{B}}}\right)$.

Nesta seção consideraremos que $X$ é um espaço métrico compacto e totalmente desconexo e $\varphi: X \rightarrow X$ é um homeomorfismo minimal sem pontos periódicos. Na Seção 8.3 do Capítulo 8 , vimos que relação orbital $R_{\varphi}$ é uma relação de equivalência étale.

Proposição 12.2.1. O grupo $B\left(X, R_{\varphi}\right)$ é gerado pelas funções da forma $\mathcal{X}_{U}-\mathcal{X}_{\varphi(U)}$, onde $U$ é um subconjunto clopen de $X$.

Prova. Lembre que a ação local associada a função $\varphi$ é a coleção

$$
\Gamma_{\varphi}=\left\{\varphi_{\mid U}^{n} \mid n \in \mathbb{Z} \text { e } U \subset X \text { é clopen }\right\}
$$

Usando o Lema 10.2.1, temos que $B\left(X, R_{\varphi}\right)$ é gerado pelas funções $\mathcal{X}_{\pi_{2}(\gamma)}$ $\mathcal{X}_{\pi_{1}(\gamma)}$, onde $\gamma=\varphi_{\mid U}^{n}=\left(U, \varphi^{n}(U)\right)$, onde $n \in \mathbb{Z}$ e $U$ é clopen. Se $n=0, \gamma$ é a 
função constante igual a 0 . Considere o caso $n \geq 1$. Definimos, para $1 \leq i \leq n$

$$
U_{i}:=\varphi^{i}(U)
$$

Então

$$
\begin{aligned}
\mathcal{X}_{\pi_{2}(\gamma)}-\mathcal{X}_{\pi_{1}(\gamma)} & =\mathcal{X}_{\varphi^{n}(U)}-\mathcal{X}_{U}=-\left(\mathcal{X}_{U}-\mathcal{X}_{\varphi^{n}(U)}\right) \\
& =-\sum_{i=0}^{n}-\mathcal{X}_{\varphi^{i}(U)}-\mathcal{X}_{\varphi^{i+1}(U)} \\
& =-\sum_{i=0}^{n} \mathcal{X}_{U_{i}}-\mathcal{X}_{\varphi\left(U_{i}\right)} .
\end{aligned}
$$

Como $\varphi$ é um homeomorfismo, $U_{i}$ é clopen para todo $1 \leq i \leq n$.

Agora, considere o caso em que $n<0$. Temos que

$$
\mathcal{X}_{\pi_{2}(\gamma)}-\mathcal{X}_{\pi_{1}(\gamma)}=\mathcal{X}_{\varphi^{n}(U)}-\mathcal{X} \varphi^{-n}\left(\varphi^{n}(U)\right)
$$

Como $-n>0$, podemos aplicar o caso feito acima, definindo para cada $1 \leq i \leq n$,

$$
V_{i}:=\varphi^{i}(U),
$$

que é um conjunto clopen, já que $U$ é clopen e $\varphi$ é um homeomorfismo. Concluímos, assim, a demonstração da proposição.

Proposição 12.2.2. Temos que $B\left(X, R_{\varphi}\right)=\left\{f-f \circ \varphi^{-1} \mid f \in C(X, \mathbb{Z})\right\}$.

Prova. Mostraremos, primeiro, que

$$
B\left(X, R_{\varphi}\right) \subset\left\{f-f \circ \varphi^{-1} \mid f \in C(X, \mathbb{Z})\right\} .
$$

Pela Proposição 12.2.1, $B\left(X, R_{\varphi}\right)$ é o conjunto gerado pelas funções $\mathcal{X}_{U}-\mathcal{X}_{\varphi(U)}$, onde $U$ é um subconjunto clopen de $X$. Então, dado $U$ subconjunto clopen de $X$, mostraremos que

$$
\mathcal{X}_{U}-\mathcal{X}_{\varphi(U)} \in\left\{f-f \circ \varphi^{-1} \mid f \in C(X, \mathbb{Z})\right\}
$$

Temos que a função $\mathcal{X}_{U} \in C(X, \mathbb{Z})$, além disso,

$$
\mathcal{X}_{U} \circ \varphi^{-1}=\mathcal{X}_{\varphi(U)}
$$

Então

$$
\mathcal{X}_{U}-\mathcal{X}_{\varphi(U)}=\mathcal{X}_{U}-\mathcal{X}_{U} \circ \varphi^{-1}
$$


Portanto,

$$
B\left(X, R_{\varphi}\right) \subset\left\{f-f \circ \varphi^{-1} \mid f \in C(X, \mathbb{Z})\right\} .
$$

Agora, mostraremos que

$$
\left\{f-f \circ \varphi^{-1} \mid f \in C(X, \mathbb{Z})\right\} \subset B\left(X, R_{\varphi}\right) .
$$

Seja $f \in C(X, \mathbb{Z})$, o conjunto $\left\{f^{-1}(\{n\})\right\}_{n \in f(X)}$ é uma partição de $X$, logo

$$
f=\sum_{n \in f(X)} n \mathcal{X}_{f^{-1}(\{n\})}
$$

Então

$$
\begin{aligned}
f-f \circ \varphi^{-1} & =\sum_{n \in f(X)} n \mathcal{X}_{f^{-1}(\{n\})}-\left(\sum_{n \in f(X)} n \mathcal{X}_{f^{-1}(\{n\})}\right) \circ \varphi^{-1} \\
& =\sum_{n \in f(X)} n\left(\mathcal{X}_{f^{-1}(\{n\})}-\mathcal{X}_{f^{-1}(\{n\})} \circ \varphi^{-1}\right) \\
& =\sum_{n \in f(X)} n\left(\mathcal{X}_{f^{-1}(\{n\})}-\mathcal{X}_{\varphi\left(f^{-1}(\{n\})\right)}\right) .
\end{aligned}
$$

Como $f^{-1}(\{n\})$ é clopen para todo $n \in f(X), f-f \circ \varphi^{-1} \in B\left(X, R_{\varphi}\right)$. Portanto

$$
B\left(X, R_{\varphi}\right)=\left\{f-f \circ \varphi^{-1} \mid f \in C(X, \mathbb{Z})\right\}
$$

Concluímos, assim, a demonstração do lema.

Lembre que um diagrama de Bratteli ordenado é próprio se possui somente um caminho infinito formado somente por arestas máximas e um caminho infinito formado somente por arestas mínimas.

Proposição 12.2.3. Considere $\mathfrak{B}=(V, E, r, s, \geq)$ um diagrama de Bratteli ordenado e próprio e $\varphi_{\mathfrak{B}}$ sua função de Bratteli-Vershik. Então

$$
B\left(X_{\mathfrak{B}}, R_{\mathfrak{B}}\right)=B\left(X, R_{\varphi_{\mathfrak{B}}}\right)
$$

Prova. Lembre que podemos ver uma relação de equivalência $R$ em um espaço $X$ como um conjunto de pares

$$
R=\left\{(x, y) \in X \times X \mid x \sim_{R} y\right\}
$$

Mostraremos primeiro que $B\left(X_{\mathfrak{B}, R_{\mathfrak{B}}}\right) \subset B\left(X, R_{\mathfrak{B}}\right)$. Usando o Teorema 6.2.1, temos que $R_{\mathfrak{B}} \subset R_{\varphi_{\mathfrak{B}}}$. Além disso, temos que $R_{\mathfrak{B}}$ é um conjunto aberto, Assim, se $\gamma$ é um homeomorfismo parcial compacto e aberto em $R_{\mathfrak{B}}$ está contido em $R_{\varphi}$. 
Como, por definição, $B(X, R)$ é o grupo gerado pelas funções $\mathcal{X}_{\pi_{2}(\gamma)}-\mathcal{X}_{\pi_{1}(\gamma)}$, onde $\gamma$ é um homeomorfismo parcial compacto e aberto, temos que

$$
B\left(X_{\mathfrak{B}}, R_{\mathfrak{B}}\right) \subset B\left(X_{\mathfrak{B}}, R_{\varphi_{\mathfrak{B}}}\right)
$$

Para mostrar a inclusão contrária, pela Proposição 12.2.1, podemos considerar um subconjunto clopen $U$ e verificar que

$$
\mathcal{X}_{U}-\mathcal{X}_{\varphi(U)} \in B\left(X_{\mathfrak{B}}, R_{\mathfrak{B}}\right)
$$

Consideraremos dois casos. O primeiro caso é quando $\mathbf{x}_{\max }$ não está no conjunto $U$. Então o homeomorfismo parcial $\gamma=\varphi_{\mid U}$ é compacto e aberto em $R_{\mathfrak{B}}$ e

$$
\mathcal{X}_{U}-\mathcal{X}_{\varphi(U)}=\mathcal{X}_{\pi_{1}(\gamma)}-\mathcal{X}_{\pi_{2}(\gamma)} \in B\left(X_{\mathfrak{B}}, R_{\mathfrak{B}}\right)
$$

Agora, suponha que $\mathbf{x}_{\max } \in U$, então $\mathbf{x}_{\max }$ não está em $X \backslash U, \operatorname{logo} \beta=\varphi_{\mid X \backslash U}$ é um homeomorfismo parcial compacto e aberto. Temos que

$$
\begin{aligned}
\mathcal{X}_{U}-\mathcal{X}_{\varphi(U)} & =\left(1-\mathcal{X}_{X \backslash U}\right)-\left(1-\mathcal{X}_{X \backslash \varphi(U)}\right) \\
& =-\left(\mathcal{X}_{X \backslash U}-\mathcal{X}_{\varphi(X \backslash U)}\right) \\
& =\mathcal{X}_{\pi_{2}(\beta)}-\mathcal{X}_{\pi_{1}(\beta)} \in B\left(X_{\mathfrak{B}}, R_{\mathfrak{B}}\right) .
\end{aligned}
$$

Concluímos, assim, que $B\left(X_{\mathfrak{B}}, R_{\mathfrak{B}}\right)=B\left(X_{\mathfrak{B}}, R_{\varphi_{\mathfrak{B}}}\right)$.

Como $D(X, R)=C(X, \mathbb{Z}) / B(X, R)$, concluímos, pela Proposição 12.2.3, que

$$
D\left(X_{\mathfrak{B}}, R_{\mathfrak{B}}\right)=D\left(X_{\mathfrak{B}}, R_{\varphi_{\mathfrak{B}}}\right) .
$$

Considere a função $\beta: D\left(X_{\mathfrak{B}}, R_{\varphi_{\mathfrak{B}}}\right) \rightarrow D\left(X_{\mathfrak{B}}, R_{\mathfrak{B}}\right)$, definida por

$$
\beta\left(f+B\left(X_{\mathfrak{B}}, R_{\varphi_{\mathfrak{B}}}\right)\right)=f+B\left(X_{\mathfrak{B}}, R_{\mathfrak{B}}\right) .
$$

Lema 12.2.4. A função $\beta$ é um isomorfismo que preserva ordem unitária.

Prova. Considere $f$ uma ordem unitária em $D\left(X_{\mathfrak{B}}, R_{\varphi_{\mathfrak{B}}}\right)^{+}$. Dada $g \in$ $D\left(X_{\mathfrak{B}}, R_{\varphi_{\mathfrak{B}}}\right)$ existe um inteiro positivo $n$ tal que $n f-g \in D\left(X_{\mathfrak{B}}, R_{\varphi_{\mathfrak{B}}}\right)^{+}$. Temos que

$$
\begin{aligned}
\beta\left(n f-g+B\left(X_{\mathfrak{B}}, R_{\varphi_{\mathfrak{B}}}\right)\right. & =n \beta\left(f+B\left(X_{\mathfrak{B}}, R_{\varphi_{\mathfrak{B}}}\right)\right)-\beta\left(g+B\left(X_{\mathfrak{B}}, R_{\varphi_{\mathfrak{B}}} /\right)\right) \\
& =n f-g+B\left(X_{\mathfrak{B}}, R_{\mathfrak{B}}\right) \geq 0 .
\end{aligned}
$$

Portanto $n f-g+B\left(X_{\mathfrak{B}}, R_{\mathfrak{B}}\right) \in D\left(X_{\mathfrak{B}}, R_{\mathfrak{B}}\right)^{+}$. 
Seja $\mathfrak{B}=(V, E, r, s, \geq)$ uma digrama de Bratteli ordenado e próprio tal que $V=\left\{v_{0}\right\}$ e $\varphi_{\mathfrak{B}}$ sua função de Bratteli-Vershik. Sejam $\alpha: D\left(X_{\mathfrak{B}}, R_{\mathfrak{B}}\right) \rightarrow$ $D(\mathfrak{B})$ o isomorfismo do Teorema 12.1 .1 e $\beta$ o isomorfismo definido na equação (12.2.1). Considere a função $\delta: D\left(X_{\mathfrak{B}}, R_{\varphi_{\mathfrak{B}}}\right)$ definida por

$$
\delta=\alpha \circ \beta .
$$

Afirmação 12.2.5. A função $\delta$ é um isomorfismo.

Como $\delta$ é composição de isomorfismos segue que $\delta$ também é um isomorfismo.

Afirmação 12.2.6. $\delta\left(D\left(X_{\mathfrak{B}}, R_{\varphi_{\mathfrak{B}}}\right)^{+}\right)=D(\mathfrak{B})^{+}$.

Considere $f \in D\left(X_{\mathfrak{B}}, R_{\varphi_{\mathfrak{B}}}\right)^{+}$, temos que $f$ é uma função positiva e $f \in B\left(X_{\mathfrak{B}}, R_{\varphi_{\mathfrak{B}}}\right)$. Como $B\left(X_{\mathfrak{B}}, R_{\varphi_{\mathfrak{B}}}\right)=B\left(X_{\mathfrak{B}}, R_{\mathfrak{B}}\right)$, obtemos que

$$
\beta\left(f+B\left(X_{\mathfrak{B}}, R_{\varphi_{\mathfrak{B}}}\right)\right)=f+B\left(X_{\mathfrak{B}}, R_{\mathfrak{B}}\right) \in D\left(X_{\mathfrak{B}}, R_{\mathfrak{B}}\right)^{+} .
$$

Portanto, $\beta\left(D\left(X_{\mathfrak{B}}, R_{\varphi_{\mathfrak{B}}}\right)^{+}\right) \subset D\left(X_{\mathfrak{B}}, R_{\mathfrak{B}}\right)^{+}$. Analogamente, obtemos que $D\left(X_{\mathfrak{B}}, R_{\mathfrak{B}}\right) \subset \beta\left(D\left(X_{\mathfrak{B}}, R_{\varphi_{\mathfrak{B}}}\right)^{+}\right.$. Assim,

$$
\beta\left(D\left(X_{\mathfrak{B}}, R_{\mathfrak{B}}\right)^{+}\right)=D\left(X_{\mathfrak{B}}, R_{\varphi_{\mathfrak{B}}}\right)^{+}
$$

Então

$$
\alpha\left(\beta\left(D\left(X_{\mathfrak{B}}, R_{\mathfrak{B}}\right)^{+}\right)\right)=\alpha\left(D\left(X_{\mathfrak{B}}, R_{\varphi_{\mathfrak{B}}}\right)^{+}\right)=D(\mathfrak{B})^{+} .
$$

Provando, assim, a afirmação.

As construções acima podem ser resumidas no seguinte resultado:

Teorema 12.2.7. Seja $\mathfrak{B}=(V, E, r, s, \geq)$ um diagrama de Bratteli ordenado e próprio tal que $V_{0}=\left\{v_{0}\right\}$ e $\varphi_{\mathfrak{B}}$ sua função de Bratteli-Vershik. Então a função

$$
\delta: D\left(X_{\mathfrak{B}}, R_{\varphi_{\mathfrak{B}}}\right) \rightarrow D(\mathfrak{B})
$$

definida na equação (12.2.1) é um isomorfismo tal que

$$
\delta\left(D\left(X_{\mathfrak{B}}, R_{\varphi_{\mathfrak{B}}}\right)^{+}\right)=D(\mathfrak{B})^{+} \quad e \quad \delta(\langle\mathbf{1}\rangle)=\left\langle v_{0}, 0\right\rangle .
$$

Prova. Só falta provar que

$$
\delta(\langle\mathbf{1}\rangle)=\left\langle v_{0}, 0\right\rangle
$$


Por definição de $\delta$ temos que,

$$
\delta(\langle\mathbf{1}\rangle)=\alpha \circ \beta(\langle\mathbf{1}\rangle)
$$

Pela definição da função $\beta$, temos que $\beta(\langle\mathbf{1}\rangle)=\mathbf{1}$. Pelo Teorema 12.1.1,

$$
\alpha(\langle\mathbf{1}\rangle)=\left\langle v_{0}, 0\right\rangle .
$$

Concluímos, assim, a demonstração do teorema. 


\section{O Teorema de Effros-Handelman-Shen}

No capítulo 11 vimos como, a partir de um diagrama de Bratteli, obter um grupo abeliano ordenado o qual foi denominado de grupo de dimensão associado ao diagrama de Bratteli.

Agora, queremos determinar quando um grupo abeliano ordenado é o grupo de dimensão de um diagrama de Bratteli. Para isso, usaremos o Teorema de Effros-Handelman-Shen.

\section{1}

\section{O Teorema de Effros-Handelman-Shen}

Precisaremos de alguns conceitos preliminares antes de enunciar o Teorema de Effros-Handelman-Shen.

Definição 13.1.1. Um grupo abeliano ordenado $\left(G, G^{+}\right)$é dito perfurado se existem um elemento a e um inteiro positivo $n$ tais que a $\notin G^{+}$e na $\in G^{+}$. Caso contrário $\left(G, G^{+}\right)$é dito sem perfuração.

Definição 13.1.2. Um grupo é dito sem torção se o único elemento que possui ordem finita é o elemento neutro.

Proposição 13.1.3. Se um grupo abeliano ordenado $\left(G, G^{+}\right)$é sem perfuração então ele é sem torção.

Prova. Considere um elemento $g \in G$ que $g$ possui ordem finita, isto é, existe inteiro $n \geq 1$ tal que $n g=0$. Temos que ver que $g$ é o elemento neutro. Como $0 \in G^{+}, n g \in G^{+}$e, por hipótese, $G$ é sem perfuração segue que $g$ está em $G^{+}$. Já que $n-1>0,(n-1) g \in G^{+}$. Como $n g=0$, o elemento $(n-1) g$ é o inverso de $n g$. Pela definição de cone positivo, temos que $G^{+} \cap\left(-G^{+}\right)=0$, então só podemos ter $g=0$. Concluímos que $G$ é livre de torção.

Seja $X$ um conjunto, considere os elementos $x, y, z, w$. Se $x \leq z, y \leq z$, $x \leq * 9+w$ e $y \leq w$, dizemos que $x, y \leq z, w$.

Definição 13.1.4. Um grupo abeliano ordenado $\left(G, G^{+}\right)$satisfaz a interpolação de Riesz se dados quaisquer $a, b, c, d$ em $G$ tais que $a, b \leq c, d$ existe um elemento e em $G$ tal que $a, b \leq e \leq c, d$. 
Dado $G$ um grupo ordenado qualquer, nem sempre podemos comparar quaisquer dois elementos. Repare que se $G$ é um grupo ordenado tal que a sua ordem é total, $G$ satisfaz a interpolação de Riesz, já que se $a, b \leq c, d$, podemos tomar o elemento $e$ como sendo o maior entre $a$ e $b$ ou o menor entre $c$ e $d$.

Considere um conjunto finito $F$. Dados dois elementos $a=\sum_{f \in F} a_{f} f$ e $b=\sum_{f \in F} b_{f} f$ em $\mathbb{Z} F$, por definição, temos que $a \leq b$ se e somente se $b-a \in \mathbb{Z} F^{+}$. Mas,

$$
b-a=\sum_{f \in F} b_{f} f-\sum_{f \in F} a_{f} f=\sum_{f \in F}\left(b_{f}-a_{f}\right) f,
$$

então $b-a \in \mathbb{Z} F^{+}$se e somente se $b_{f}-a_{f} \geq 0$ para todo $f \in F$, isto é, se e somente se $b_{f} \geq a_{f}$ para todo $f \in F$. Como $\mathbb{Z}$ é totalmente ordenado, para todo $f \in F$ o maior número entre $a_{f}$ e $b_{f}$ pode ser determinado.

Observação 13.1.5. Considere $F$ um conjunto finito, então o grupo $\left(\mathbb{Z} F, \mathbb{Z} F^{+}\right)$ satisfaz a interpolação de Riesz.

Prova. Definimos

$$
a=\sum_{f \in F} a_{f} f \in \mathbb{Z} F
$$

e similarmente os elementos $b, c, d$. Se $a, b \leq c, d$, podemos definir

$$
e=\sum_{f \in F} \max \left\{a_{f}, b_{f}\right\} f
$$

Logo, $a, b \leq e \leq c, d$.

Proposição 13.1.6. Considere $A$ um conjunto finito. Então $\mathbb{Z} A$ é enumerável $e\left(\mathbb{Z} A, \mathbb{Z} A^{+}\right)$é sem perfuração e satisfaz a interpolação de Riesz.

Prova. Pela Observação 13.1.5, ( $\left.\mathbb{Z} A, \mathbb{Z} A^{+}\right)$satisfaz a interpolação de Riesz. Como $A$ é finito e $\mathbb{Z}$ é enumerável, segue que $\mathbb{Z} A$ é enumerável. Falta mostrar que $\left(\mathbb{Z} A, \mathbb{Z} A^{+}\right)$é sem perfuração. Seja a um elemento de $\mathbb{Z} A$ que não está em $\mathbb{Z} A^{+}$, então podemos escrever

$$
a=\sum_{k=1}^{\ell} i_{k} a_{k}, \quad \text { existe } j \text { tal que } i_{j}<0
$$

Para todo inteiro positivo $n$, temos $n i_{j}<0$, então na não está em $\mathbb{Z} A^{+}$. Portanto $\left(\mathbb{Z} A, \mathbb{Z} A^{+}\right)$é sem perfuração. 
Teorema 13.1.7 (Effros-Handelman-Shen). Considere um grupo abeliano ordenado $\left(G, G^{+}\right)$. Existe um diagrama de Bratteli $\mathfrak{B}=(V, E, r, s)$ tal que $\left(G, G^{+}\right) \cong\left(D(\mathfrak{B}), D(\mathfrak{B})^{+}\right)$se e somente se $\left(G, G^{+}\right)$satisfaz as seguintes condições:

1. G é enumerável;

2. $\left(G, G^{+}\right)$é sem perfuração;

3. $\left(G, G^{+}\right)$satisfaz a interpolação de Riesz.

Além disso, se $\left(G, G^{+}\right)$satisfaz as três condições acima e possui uma ordem unitária $u$, então $V_{0}$ pode ser escolhido como um conjunto unitário $V_{0}=\left\{v_{0}\right\}$ e o isomorfismo entre $G$ e $D(\mathfrak{B})$ para levar u em $\left\langle v_{0}, 0\right\rangle$.

Prova.

Lema 13.1.8. Seja $\left\{G_{n}\right\}_{n \geq 0}$ uma sequência de grupos, tal que para todo $n \geq 0$, $G_{n}$ é enumerável, sem perfuração e satisfaz a interpolação de Riesz. Suponha que existe uma sequência de homomorfismos $\left\{\alpha_{n}\right\}_{n \geq 1}$ tal que $\alpha_{n}: G_{n-1} \rightarrow G_{n}$, então $\left(G_{\infty}, \alpha_{\infty}\right)$ é enumerável, sem perfuração e satisfaz a interpolação de Riesz.

Prova. Podemos identificar o elemento $\langle a, n\rangle$ com o elemento $a \in G_{n}$. Portanto $\left(G_{\infty}, \alpha_{\infty}\right)$ é enumerável.

Agora, se $\langle a, n\rangle$ não está em $\left(G_{\infty}, \alpha_{\infty}\right)^{+}$, temos que para todo $(b, k)$ tal que $(b, k) \sim(a, n), b$ não está em $\mathbb{Z} G_{k}^{+}$. Logo para todo $\ell>0, \ell\langle b, k\rangle$ não está em $\left(G_{\infty}, \alpha_{\infty}\right)^{+}$. Portanto, $\left(G_{\infty}, \alpha_{\infty}\right)$ é sem perfuração.

Por fim, considere os elementos $\left\langle a_{1}, n_{1}\right\rangle, \quad\left\langle a_{2}, n_{2}\right\rangle, \quad\left\langle b_{1}, m_{1}\right\rangle$ e $\left\langle b_{2}, m_{2}\right\rangle$ tais que $\left\langle a_{1}, n_{1}\right\rangle,\left\langle a_{2}, n_{2}\right\rangle \leq\left\langle b_{1}, m_{1}\right\rangle,\left\langle b_{2}, m_{2}\right\rangle$. Existe inteiro $N>\max \left\{n_{1}, n_{2}, m_{1}, m_{2}\right\}$ tal que

$$
\left\langle a_{i}, n_{i}\right\rangle=\left\langle\alpha_{n_{i}, N}\left(a_{i}\right), N\right\rangle \quad \text { e }\left\langle b_{i}, m_{i}\right\rangle=\left\langle\alpha_{m_{i}, N}\left(b_{i}\right), N\right\rangle \text {, para } 1 \leq i \leq 2 .
$$

Então

$$
\left\langle\alpha_{n_{i}, N}\left(a_{i}\right), N\right\rangle \leq\left\langle\alpha_{m_{i}, N}\left(b_{i}\right), N\right\rangle
$$

Portanto,

$$
\alpha_{n_{1}, N}\left(a_{1}\right), \alpha_{n_{2}, N}\left(a_{2}\right) \leq \alpha_{m_{1}, N}\left(m_{1}\right), \alpha_{m_{2}, N}\left(b_{2}\right)
$$

em $G_{N}$. Como $G_{N}$ satisfaz a interpolação de Riesz é possível encontrar $e \in G_{N}$ tal que

$$
\alpha_{n_{1}, N}\left(a_{1}\right), \alpha_{n_{2}, N}\left(a_{2}\right) \leq e \leq \alpha_{m_{1}, N}\left(m_{1}\right), \alpha_{m_{2}, N}\left(b_{2}\right) .
$$

Concluímos, assim, a demonstração do lema. 
Como para cada $n \geq 0, V_{n}$ é um conjunto finito, usando a Proposição 13.1.6, temos que as propriedades (1), (2), (3) valem para $\mathbb{Z} V_{n}$. Pelo Lema 13.1.8, as propriedades também valem para o grupo $\left(\mathbb{Z} V_{\infty}, h_{\infty}\right)$ que, por definição, é o grupo de dimensão do diagrama de Bratteli $\mathfrak{B}$. Provamos, então a ida do teorema.

\subsection{1}

\section{O Teorema de Shen}

Nesta seção, provaremos o Teorema de Shen que será usado na demonstração da volta do Teorema de Effros-Handelman-Shen.

Proposição 13.1.9. Considere A um conjunto finito. Então todo subgrupo $H$ de $\mathbb{Z} A$ é finitamente gerado.

Prova. Definimos $\# A=n$. A prova será por indução em $n$. No caso $n=1$, $\mathbb{Z} A=\mathbb{Z}$. Como todo subgrupo de $\mathbb{Z}$ é cíclico ou $\{0\}$, temos que $H$ possui somente um gerador ou é $\{0\}$. Assuma que o resultado é válido para algum $k \geq 1$. Mostraremos que o lema é verdadeiro para o caso $n=k+1$. Como $A$ é finito podemos escrever

$$
A=\left\{a_{1}, \ldots, a_{k}, a_{k+1}\right\}
$$

Considere $H$ um subgrupo de $\mathbb{Z} A$ e o conjunto $B=\left\{a_{1}, \ldots, a_{k}\right\}$. Definimos o homomorfismo $f: \mathbb{Z} A \rightarrow \mathbb{Z} B$ tal que

$$
f\left(n_{1} a_{1}+\cdots+n_{k+1} a_{k+1}\right)=n_{1} a_{1}+\cdots+n_{k} a_{k} .
$$

Temos que $f(H)$ é um subgrupo de $\mathbb{Z} B$. Pela hipótese de indução, $f(H)$ é finitamente gerado. Portanto existe um número finito de geradores, digamos, $f\left(h_{1}\right), \ldots, f\left(h_{d}\right)$, onde $h_{1}, \ldots, h_{d}$ são elementos de $H$ e $d \leq k$.

Lema 13.1.10. $\operatorname{Ker}(f)=Z a_{k+1}$.

Prova. Seja $n_{k+1} a_{k+1} \in \mathbb{Z} a_{k+1}$, temos que $f\left(n_{k+1} a_{k+1}\right)=0$. Agora seja $n_{1} a_{1}+\cdots+n_{k+1} a_{k+1} \in \operatorname{Ker}(f)$, então

$$
n_{1} a_{1}+\cdots+n_{k} a_{k}=0
$$

Logo, $n_{i}=0$ para todo $1 \leq i \leq k$ e portanto $n_{1} a_{1}+\cdots+n_{k+1} a_{k+1} \in \mathbb{Z} a_{k+1}$.

Pelo lema acima, $H \cap \operatorname{Ker}(f) \subset \mathbb{Z} a_{k+1}$ é o subgrupo $\{0\}$ ou um grupo cíclico. No primeiro caso, $f_{\mid H}$ é um isomorfismo entre $H$ e $f(H)$ e portanto $H$ é 
gerado pelos elementos $h_{1}, \ldots h_{d}$. Agora, se $H \cap \operatorname{Ker}(f)$ for cíclico, denotamos $h_{d+1}$ como o gerador de $H \cap \operatorname{Ker}(f)$.

O lema a seguir finaliza a demonstração da proposição.

Lema 13.1.11. H é o subgrupo gerado pelos elementos $h_{1}, \ldots, h_{d+1}$.

Prova. Seja $h \in H$, então $f(h) \in f(H)$, logo existem inteiros $n_{1}, \ldots n_{d}$, tais que

$$
f(h)=\sum_{i=1}^{d} n_{i} f\left(h_{i}\right)
$$

Então,

$$
f(h)-\sum_{i=1}^{d} n_{i} f\left(h_{i}\right)=0 \Rightarrow f\left(h-\sum_{i_{1}}^{d} n_{i} h_{i}\right)=0 \Rightarrow h-\sum_{i=1}^{d} n_{i} h_{i} \in \operatorname{Ker}(f) .
$$

Portanto, $h-\sum_{i=1}^{d} n_{i} h_{i} \in H \cap \operatorname{Ker}(f)$, então existe inteiro $n_{d+1}$ tal que $h-\sum_{i=1}^{d} n_{i} h_{i}=n_{d+1} h_{d+1}$. Logo $h=\sum_{i=1}^{d+1} n_{i} h_{i}$. Verificamos, assim, que o lema é verdadeiro.

Proposição 13.1.12. Seja $\left(G, G^{+}\right)$um grupo abeliano ordenado que satisfaz a interpolação de Riesz. Considere $a, b_{1}, \ldots, b_{d}$ elementos de $G^{+}$. Se

$$
a \leq b_{1}+b_{2}+\cdots+b_{d}
$$

então existem $a_{1}, \ldots, a_{d} \in G^{+}$tais que

$$
a_{1}+a_{2}+\cdots+a_{d}=a \quad \text { e } \quad a_{i} \leq b_{i} \text { para todo } 1 \leq i \leq d
$$

Prova. A prova é por indução em $d$. Para o caso $d=1$ basta tomar $a_{1}=a$. Suponha, por indução, que o lema seja verdadeiro para $d=k$. Provaremos que o lema vale para $d=k+1$. Considere $a, b_{1}, \ldots, b_{k}, b_{k+1} \mathrm{em} G^{+}$satisfazendo a hipótese do lema. Temos que

$$
0, a-b_{k+1} \leq a, b_{1}+\cdots+b_{k}
$$

Como a interpolação de Riesz é satisfeita, existe $c$ tal que

$$
0, a-b_{k+1} \leq c \leq a, b_{1}+\cdots+b_{k}
$$

Em particular,

$$
c \leq b_{1}+\cdots+b_{k}
$$


Usando a hipótese de indução em $c$, existem $a_{1}, \ldots, a_{k}$ em $G^{+}$tais que

$$
a_{1}+\cdots+a_{k}=c \quad \text { e } \quad a_{i} \leq b_{i} \text { para todo } 1 \leq i \leq k .
$$

Definimos $a_{k+1}=a-c$. Como, pela equação (13.1.1), $c \leq a$ temos que $a_{k+1} \in G^{+}$. Além disso,

$$
a-c \leq a-\left(a-b_{k+1}\right)=b_{k+1} .
$$

Por fim,

$$
a_{1}+\cdots+a_{k+1}=c+(a-c)=a .
$$

Concluímos, assim a demonstração do lema.

Teorema 13.1.13 (Shen). Considere A um conjunto finito e $\left(G, G^{+}\right)$um grupo abeliano ordenado que é sem perfuração e satisfaz a interpolação de Riesz. Se existe um homomorfismo positivo $\alpha:\left(\mathbb{Z} A, \mathbb{Z} A^{+}\right) \rightarrow\left(G, G^{+}\right)$então existem um conjunto finito $B$ e homomorfismos positivos $\eta:\left(\mathbb{Z} A, \mathbb{Z} A^{+}\right) \rightarrow\left(\mathbb{Z} B, \mathbb{Z} B^{+}\right)$e $\beta:\left(\mathbb{Z} B, \mathbb{Z} B^{+}\right) \rightarrow\left(G, G^{+}\right)$, tais que

$$
\operatorname{Ker}(\eta)=\operatorname{Ker}(\alpha) \quad \text { e } \beta \circ \eta=\alpha
$$

Prova. Começaremos demonstrando um caso particular do teorema.

Proposição 13.1.14 (Shen). Considere A um conjunto finito e $\left(G, G^{+}\right)$um grupo abeliano ordenado, sem perfuração e que satisfaz a interpolação de Riesz. Suponha que existem um homomorfismo positivo

$$
\alpha:\left(\mathbb{Z} A, \mathbb{Z} A^{+}\right) \rightarrow\left(G, G^{+}\right)
$$

e $u \in \mathbb{Z} A$ tal que $\alpha(u)=0$. Então existem um conjunto finito $B$ e homomorfismos positivos

$$
\eta:(\mathbb{Z} A, \mathbb{Z} A)^{+} \rightarrow\left(\mathbb{Z} B, \mathbb{Z} B^{+}\right) \quad \text { e } \beta:\left(\mathbb{Z} B, \mathbb{Z} B^{+}\right) \rightarrow\left(G, G^{+}\right)
$$

tais que $\eta(u)=0$ e $\beta \circ \eta=\alpha$.

Prova. Seja $u=\sum_{a \in A} n_{a} a$. Definimos

$$
n(u)=\max \left\{\left|n_{a}\right| \mid a \in A\right\} \quad \text { e } m(u)=\#\left\{a \in A|| n_{a} \mid=n(u)\right\} .
$$

Consideraremos dois casos. O caso trivial acontece quando $n(u)=0$. Neste caso $u=0$. Assim, basta tomar $B=A, \beta=\alpha$ e $\eta$ como a identidade. 
Então

$$
\eta(u)=\operatorname{id}(0)=0 \quad \text { e } \quad \beta \circ \eta=\alpha \circ \mathrm{id}=\alpha .
$$

Agora, considere que $n(u)>0$. Definimos

$$
A^{+}=\left\{a \in A \mid n_{a}>0\right\} \quad \text { e } \quad A^{-}=\left\{a \in A \mid n_{a}<0\right\}
$$

Repare que podem existir elementos em $A$ que não estão em $A^{+}$nem em $A^{-}$.

Lema 13.1.15. Se $A^{-}=\emptyset$ então $\alpha(a)=0$ para todo $a \in A^{+}$.

Prova. Como $A^{-}=\emptyset$, temos que

$$
0=\alpha(u)=\sum_{a \in A^{+}} n_{a} \alpha(a)
$$

Como $\alpha$ é um homomorfismo positivo, para cada $a \in A^{+}, \alpha(a) \in G^{+}$.

Fixe qualquer $a_{i} \in A^{+}$,

$$
0=\alpha\left(a_{i}-a_{i}\right)=\alpha(u) \Rightarrow \alpha\left(a_{i}\right)-\alpha\left(a_{i}\right)=\sum_{a \in A^{+}} n_{a} \alpha(a) .
$$

Então,

$$
-\alpha\left(a_{i}\right)=n_{a_{i}} \alpha\left(a_{i}\right)-\alpha\left(a_{i}\right)+\sum_{a \in A^{+}, a \neq a_{i}} n_{a} \alpha(a) .
$$

Portanto,

$$
-\alpha\left(a_{i}\right)=\left(n_{a_{i}}-1\right) \alpha\left(a_{i}\right)+\sum_{a \in A^{+}, a \neq a_{i}} n_{a} \alpha(a) .
$$

Como $n_{a_{i}}-1 \geq 0$ e $\alpha(a) \in G^{+}$para todo $a \in A^{+}$, segue que $-\alpha\left(a_{i}\right) \in G^{+}$. Assim, $\alpha\left(a_{i}\right)$ e $-\alpha\left(a_{i}\right)$ estão em $G^{+}$. Pela definição de cone positivo $G^{+} \cap\left(-G^{+}\right)=\{0\}$, temos que $\alpha\left(a_{i}\right)=0$. Como $a_{i}$ é qualquer elemento de $A^{+}$, segue que $\alpha(a)=0$ para todo $a \in A^{+}$.

Provaremos, agora a Proposição 13.1.14 no caso em que $A^{-1}=\emptyset$. Definimos,

$$
B=A-A^{+}, \quad \eta\left(\sum_{a \in A} m_{a} a\right)=\sum_{a \in B} m_{a} a \quad \text { e } \quad \beta=\alpha_{\mid \mathbb{Z} B} .
$$

Dessa forma,

$$
\beta \circ \eta\left(\sum_{a \in A} m_{a} a\right)=\alpha_{\mid \mathbb{Z} B}\left(\sum_{a \in B} m_{a} a\right)=\sum_{a \in B} m_{a} \alpha(a) .
$$

Por outro lado,

$$
\alpha\left(\sum_{a \in A} m_{a} a\right)=\sum_{a \in B} m_{a} \alpha(a)+\sum_{a \in A^{+}} m_{a} \alpha(a)=\sum_{a \in B} m_{a} \alpha(a) .
$$


Portanto $\beta \circ \eta=\alpha$. Como $A^{-}=\emptyset$, temos que

$$
u=\sum_{a \in A^{+}} n_{a} a
$$

Então, $u$ não possui coeficientes que estão em $B$, e, portanto, $\eta(u)=0$. Provamos a proposição para o caso em que $A^{-}=\emptyset$.

O caso em que $A^{+}=\emptyset$ segue substituindo $u$ por $-u$.

Falta considerar o caso em que $A^{+}$e $A^{-}$são ambos não vazios. Para isto, reduziremos ao caso em que $A^{+}$ou $A^{-}$é vazio. Como $A^{+}$e $A^{-}$é não vazio, $\alpha(u)=0$ implica que

$$
\sum_{a \in A^{+}} n_{a} \alpha(a)=\sum_{a \in A^{-}}\left(-n_{a}\right) \alpha(a) \in G^{+}
$$

Faremos o caso em que

$$
n(u)=\max \left\{n_{a} \mid a \in A^{+}\right\} \geq \max \left\{-n_{a} \mid a \in A^{-}\right\} .
$$

O caso contrário é feito substituindo $u$ por $-u$.

Fixe $a_{0}$ em $A^{+}$tal que $n_{a_{0}}=n(u)$. Temos que

$$
n_{a_{0}} \alpha\left(a_{0}\right) \leq \sum_{a \in A^{+}} n_{a} \alpha(a)=\sum_{a \in A^{-}}\left(-n_{a}\right) \alpha(a) \leq n_{a_{0}} \sum_{a \in A^{-}} \alpha(a)
$$

Portanto,

$$
\alpha\left(a_{0}\right) \leq \sum_{a \in A^{-}} \alpha(a)
$$

Como, por hipótese, $\left(G, G^{+}\right)$é sem perfurações, temos que $\alpha\left(a_{0}\right)$ e $\sum_{a \in A^{-}} \alpha(a) \in$ $G^{+}$. Agora, usando a interpolação de Riesz e a Proposição 13.1.12 para

$$
a=a_{0} \text { e } b_{i}=\alpha(a) \text { onde } a \in A^{-} \text {e } 1 \leq i \leq \#\left(A^{-}\right),
$$

encontramos para cada $a \in A^{-}$um $g_{a} \in G^{+}$tal que $g_{a} \leq \alpha(a)$ e

$$
\alpha\left(a_{0}\right)=\sum_{a \in A^{-}} g_{a}
$$

Considere uma cópia conjunto $A^{-}$denotada da seguinte forma:

$$
C_{A}=\left\{c_{a} \mid c_{a}=a \text {, onde } a \in A^{-}\right\} .
$$


Note que se $a_{1} \neq a_{2}$, então $c_{a_{1}} \neq c_{a_{2}}$. Definimos o conjunto auxiliar

$$
B_{1}=\left(A-\left\{a_{0}\right\}\right) \bigcup C_{A}
$$

e as funções $\eta_{1}: \mathbb{Z} A \rightarrow \mathbb{Z} B_{1}$ e $\beta_{1}: \mathbb{Z} B_{1} \rightarrow G$ como se segue:

$$
\eta_{1}(a)=\left\{\begin{array}{l}
\sum_{a \in A^{-}} c_{a}, \quad a=a_{0}, \\
a, \quad a \in A \backslash\left(\left\{a_{0}\right\} \cup A^{-}\right), \\
a+c_{a}, \quad a \in A^{-}
\end{array}\right.
$$

$\mathrm{e}$

$$
\beta_{1}(a)=\left\{\begin{array}{l}
\alpha(a), \quad a \in A \backslash\left\{a_{0}\right\}, \\
\alpha(a)-g_{a}, \quad a \in A^{-}, \\
g_{a}, \quad \text { se } \quad a=c_{a} .
\end{array}\right.
$$

Denotaremos o conjunto $A \backslash\left(\left\{a_{0}\right\} \cup A^{-}\right)$por $A^{\prime}$

Lema 13.1.16. As funções $\eta_{1}$ e $\beta_{1}$ são homomorfismos positivos.

Prova. Mostraremos que $\eta_{1}$ é um homomorfismo positivo. A demonstração de que $\beta_{1}$ é um homomorfismo positivo é análoga. Sejam dois elementos $x_{1}=\sum_{a \in A} m_{a}^{1} a$ e $x_{2}=\sum_{a \in A} m_{a}^{2} a$. Temos que

$$
\begin{aligned}
\eta_{1}\left(x_{1}+x_{2}\right) & =\eta_{1}\left(\sum_{a \in A}\left(m_{a}^{1}+m_{a}^{2}\right) a\right) \\
& =\left(m_{\left(a_{0}\right)}^{1}+m_{\left(a_{0}\right)}^{2}\right) \eta_{1}\left(a_{0}\right)+\sum_{a \in A^{\prime}}\left(m_{a}^{1}-m_{a}^{2}\right) \eta_{1}(a) \\
& +\sum_{a \in A^{-}}\left(m_{a}^{1}+m_{a}^{2}\right) \eta_{1}(a) \\
& =m_{\left(a_{0}\right)}^{1} \eta_{1}\left(a_{0}\right)+\sum_{a \in A^{\prime}} m_{a}^{1} \eta_{1}(a)+\sum_{a \in A^{-}} m_{a}^{1} \eta_{1}(a) \\
& +m_{\left(a_{0}\right)}^{2} \eta_{1}\left(a_{0}\right)+\sum_{a \in A^{\prime}} m_{a}^{2} \eta_{1}(a)+\sum_{a \in A^{-}} m_{a}^{2} \eta_{1}(a) \\
& =\eta_{1}\left(x_{1}\right)+\eta_{1}\left(x_{2}\right) .
\end{aligned}
$$

Portanto, $\eta_{1}$ é um homomorfismo. Mostraremos que $\eta_{1}$ é positivo. Considere um elemento $x=\sum_{a \in A} m_{a} a \in \mathbb{Z} A^{+}$. Por definição de $\mathbb{Z} A^{+}, m_{a} \geq 0$ para todo $a \in A$, temos que

$$
\eta_{1}(x)=\sum_{a \in A, a \neq a_{0}} m_{a} a+\sum_{a \in A^{-}}\left(m_{a_{0}}+m_{a}\right) c_{a}
$$

Como $m_{a} \geq 0$ e $m_{a_{0}}+m_{a}>0$ para todo $a \in A$, temos que $\eta_{1}(x) \in \mathbb{Z} B_{1}^{+}$. Portanto, $\eta_{1}$ é um homomorfismo positivo. 
Lema 13.1.17. $\beta_{1} \circ \eta_{1}=\alpha$.

Prova. Considere $x=\sum_{a \in A} m_{a} a$ um elemento de $\mathbb{Z} A$. Temos que

$$
\begin{aligned}
\beta_{1} \circ \eta_{1}\left(\sum_{a \in A} m_{a} a\right) & =\beta_{1}\left(m_{a_{0}} \sum_{a \in A^{-}} c_{a}+\sum_{a \in A^{\prime}} m_{a} a+\sum_{a \in A^{-}} m_{a_{0}}\left(a+c_{a}\right)\right) \\
& =\beta_{1}\left(\sum_{a \in A^{-}} m_{a} a+\sum_{a \in A^{\prime}} m_{a} a+\sum_{a \in A^{-}}\left(m_{a}+m_{a_{0}}\right) c_{a}\right) \\
& =\sum_{a \in A^{-}} m_{a}\left(\alpha(a)-g_{a}\right)+\sum_{a \in A^{\prime}} m_{a} \alpha(a)+\sum_{a \in A^{-}}\left(m_{a}+m_{a_{0}}\right) g_{a} \\
& =\sum_{a \in A^{-}}\left(m_{a} \alpha(a)-m_{a} g_{a}+m_{a} g_{a}+m a_{0} g_{a}\right)+\sum_{a \in A^{\prime}} m_{a} \alpha(a) \\
& =\sum_{a \in A^{-}} m_{a} \alpha(a)+\sum_{a \in A^{-}} m_{a_{0}} g_{a}+\sum_{a \in A^{\prime}} m_{a} \alpha(a) \\
& =\sum_{a \in A^{-}} m_{a} \alpha(a)+m_{a_{0}} \alpha\left(a_{0}\right)+\sum_{a \in A^{\prime}} m_{a} \alpha(a) \\
& =\sum_{a \in A} m_{a} \alpha(a)=\alpha(x) .
\end{aligned}
$$

Provamos, assim, o lema.

Agora, a expectativa é que encontrássemos que $\eta_{1}(u)=0$. Porém, isso não irá ocorrer necessariamente. Veremos qual é a solução.

Lema 13.1.18. Considere a função $\eta_{1}$, o elemento u e os números $m(u)$ e $n(u)$. Se verifica que $n\left(\eta_{1}(u)\right) \leq n(u)$. Se $n\left(\eta_{1}(u)\right)=n(u)$, então $m\left(\eta_{1}(u)\right)<m(u)$.

Prova. Temos que

$$
\begin{aligned}
\eta_{1}(u) & =\eta_{1}\left(n_{a_{0}} a_{0}+\sum_{a \in A^{+}, a \neq a_{0}} n_{a} a+\sum_{a \in A^{-}} n_{a} a\right) \\
& =\left(n_{a_{0}} \eta_{1}\left(a_{0}\right)+\sum_{a \in A^{+}, a \neq a_{0}} n_{a} \eta_{1}(a)+\sum_{a \in A^{-}} n_{a} \eta_{1}(a)\right) \\
& =\left(n_{a_{0}} c_{a}+\sum_{a \in A^{+}, a \neq a_{0}} n_{a} a+\sum_{a \in A^{-}} n_{a}\left(a+c_{a}\right)\right) \\
& =\sum_{a \in A^{+} \cup A^{-}, a \neq a_{0}} n_{a} a+\sum_{a \in A^{-}}\left(n_{a}+n_{a_{0}}\right) c_{a} .
\end{aligned}
$$

Repare que não há mais o termo $a_{0}$ nem o coeficiente $n_{a_{0}}$. Analisaremos os termos que restaram. Os coeficientes do primeiro somatório não se alteraram. Os coeficientes do segundo somatório são $n_{a}, n_{a_{0}}$, onde $a \in A^{-}$. Como, neste caso, $a \in A^{-}$e $n(u)=n_{a_{0}}$, temos que $0>n_{a} \geq-n_{a_{0}}$. Então para cada $c_{a}$, temos que $0 \leq n_{a}+n_{a_{0}}<n_{a_{0}}$. Portanto $n\left(\eta_{1}(u)\right) \leq n(u)$. Além disso, como não há mais o termo $a_{0}$, então, se $n\left(\eta_{1}(u)\right)=n(u)$ segue que $m\left(\eta_{1}(u)\right)<m(u)$. 
Definimos, $\beta_{0}=\alpha$ e indutivamente, o conjunto $B_{i}$ e os homomorfismo positivos $\eta_{i}:\left(\mathbb{Z} B_{i-1}, \mathbb{Z} B_{i-1}^{+}\right) \rightarrow\left(\mathbb{Z} B_{i}, \mathbb{Z} B_{i}^{+}\right), \beta_{k}:\left(\mathbb{Z} B_{i}, \mathbb{Z} B_{i}^{+}\right) \rightarrow\left(G, G^{+}\right)$, tais que

$$
\beta_{i} \circ \eta_{k}=\beta_{k-1}
$$

Pelo Lema 13.1.18,

$$
n\left(\eta_{i} \circ \cdots \circ \eta_{1}(u)\right) \leq n(u) .
$$

Então, existe $k>0$, tal que

$$
n\left(\eta_{i} \circ \cdots \circ \eta_{1}(u)\right)=0
$$

ou na etapa $k$, temos que $A^{+}$ou $A^{-}$é o conjunto vazio. Em qualquer um dos casos, obtemos que

$$
\eta_{i} \circ \cdots \circ \eta_{1}(u)=0
$$

Definimos, então

$$
B=B_{k}, \quad \eta=\eta_{k} \circ \cdots \circ \eta_{1} \text { e } \beta=\beta_{k}
$$

Então,

$$
\eta(u)=\eta_{k} \circ \cdots \circ \eta_{1}(u)=0
$$

Além disso,

$$
\begin{aligned}
\beta \circ \eta & =\beta_{k} \circ\left(\eta_{k} \circ \eta_{k-1} \circ \cdots \circ \eta_{1}\right)=\left(\beta_{k} \circ \eta_{k}\right) \circ \eta_{k-1} \circ \cdots \circ \eta_{1} \\
& =\left(\beta_{k-1} \circ \eta_{k-1}\right) \circ \eta_{k-2} \circ \cdots \circ \eta_{1}=\cdots=\beta_{1} \circ \eta_{1}=\alpha .
\end{aligned}
$$

Concluímos, assim, a demonstração da proposição.

Estamos prontos para fazer a demonstração do Teorema 13.1.13.

Como $\operatorname{Ker}(\alpha)$ é um subgrupo de $\mathbb{Z} A$ e $A$ é um conjunto finito, pela Proposição $13.1 .9, \operatorname{Ker}(\alpha)$ é finitamente gerado. Observe que se $\operatorname{Ker}(\alpha)$ é gerado somente por um elemento $u$, o problema já está resolvido. Suponha que os geradores de $\operatorname{Ker}(\alpha)$ sejam $u_{1}, \ldots, u_{k}$. Como $\alpha$ é um homomorfismo positivo e $\alpha\left(u_{1}\right)=0$, usando a Proposição 13.1 .14 encontramos conjunto $B_{1} \mathrm{e}$ homomorfismo positivos $\eta_{1}: \mathbb{Z} A \rightarrow \mathbb{Z} B_{1}$ e $\beta_{1}: \mathbb{Z} B_{1} \rightarrow G$ tais que $\beta_{1} \circ \eta_{1}=\alpha$ e $\eta_{1}\left(u_{1}\right)=0$.

Lema 13.1.19. Para cada $1<i \leq k$, existem conjuntos $B_{i}$ e homomorfismos positivos $\eta_{i}: \mathbb{Z} B_{i-1} \rightarrow \mathbb{Z} B_{i}$ e $\beta_{i}: \mathbb{Z} B_{i} \rightarrow G$ tais que $\beta_{i} \circ \eta_{i}=\beta_{i-1}$ e

$$
\eta_{i}\left(\eta_{i-1} \circ \cdots \circ \eta_{1}\left(u_{i}\right)\right)=0
$$


Em particular, se $j<i$

$$
\eta_{i}\left(\eta_{i-1} \circ \cdots \circ \eta_{1}\left(u_{j}\right)\right)=0
$$

Prova. A demonstração será por indução. A função $\beta_{1}$ é um homomorfismo positivo positivo. Além disso,

$$
\beta_{1}\left(\eta_{1}\left(u_{2}\right)\right)=\alpha\left(u_{2}\right)=0
$$

já que, por hipótese, $u_{2}$ é um dos geradores de $\operatorname{Ker}(\alpha)$. Usando a Proposição 13.1.14, existem conjunto $B_{2}$ e homomorfismo positivos $\eta_{2}: \mathbb{Z} B_{1} \rightarrow \mathbb{Z} B_{2}$ e $\beta_{2}: \mathbb{Z} B_{2} \rightarrow G$ tais que

$$
\beta_{2} \circ \eta_{2}=\beta_{1} \quad \text { e } \quad \eta_{2} \circ \eta_{1}\left(u_{2}\right)=0
$$

Suponha que para algum $1<i<k$ já definimos o conjunto $B_{i}$ e os homomorfismos $\eta_{i}: \mathbb{Z} B_{i-1} \rightarrow \mathbb{Z} B_{i}$ e $\beta_{i}: \mathbb{Z} B_{i} \rightarrow G$ tais que

$$
\beta_{i} \circ \eta_{i}=\beta_{i-1} \quad \text { e } \quad \eta_{i} \circ \cdots \circ \eta_{1}\left(u_{i}\right)=0 .
$$

Temos que $\beta_{i}$ é um homomorfismo positivo e que

$$
\beta_{i} \circ\left(\eta_{i} \circ \eta_{i-1} \circ \cdots \circ \eta_{1}\left(u_{i}\right)\right)=\beta_{i-1} \circ\left(\eta_{i-1} \circ \ldots \eta_{1}\left(u_{i}\right)\right)=\cdots=\alpha\left(u_{i}\right)=0 .
$$

Usando a Proposição 13.1.14, encontramos conjunto $B_{i+1}$ e homomorfismos positivos $\eta_{i+1}: \mathbb{Z} B_{i} \rightarrow \mathbb{Z} B_{i+1}$ e $\beta_{i}: \mathbb{Z} B_{i+1} \rightarrow G$ tais que

$$
\beta_{i+1} \circ \eta_{i+1}=\beta_{i} \quad \text { e } \quad \eta_{i+1} \circ \cdots \circ \eta_{1}\left(u_{i+1}\right)=0 .
$$

concluímos, assim, a demonstração do lema.

Temos o seguinte diagrama:

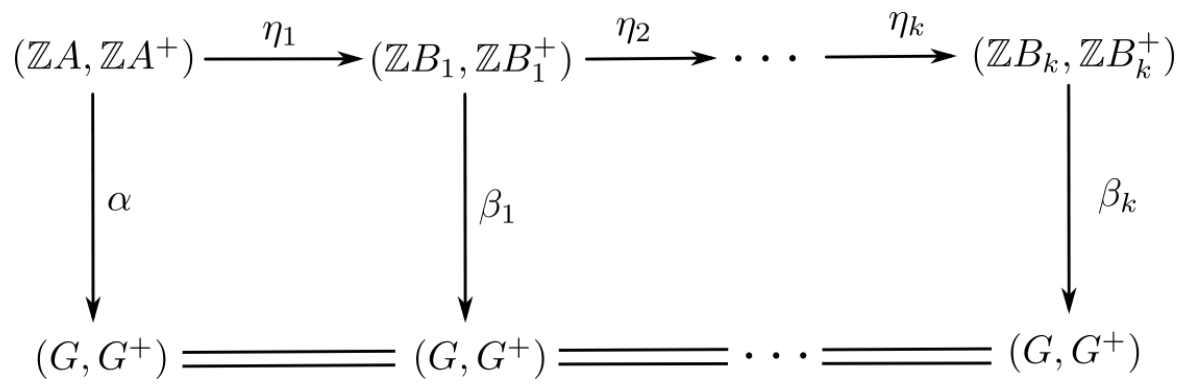

Figura 13.1: Diagrama comutativo 
Definimos, então, $B=B_{k}, \eta=\eta_{k} \circ \cdots \circ \eta_{1}$ e $\beta=\beta_{k}$.

Lema 13.1.20. $\beta \circ \eta=\alpha$.

Prova. A demonstração será por indução em $k$. O caso $k=1$ segue diretamente do Lema 13.1.14. Suponha, por indução que a afirmação seja verdadeira para $k=n$. Mostraremos que a afirmação é verdadeira para $k=n+1$. Considere a composição

$$
\begin{aligned}
\beta \circ \eta & =\beta_{n+1} \circ\left(\eta_{n+1} \circ \eta_{n} \circ \cdots \circ \eta_{1}\right)=\left(\beta_{n+1} \circ \eta_{n+1}\right) \circ\left(\eta_{n} \circ \cdots \circ \eta_{1}\right) \\
& =\beta_{n} \circ \eta_{n} \circ \cdots \circ \eta_{1}=\alpha .
\end{aligned}
$$

Finalizamos a demonstração do lema.

Falta mostrar que $\operatorname{Ker}(\alpha)=\operatorname{Ker}(\eta)$. Como $\beta \circ \eta=\alpha$, temos que $\operatorname{Ker}(\eta) \subset \operatorname{Ker}(\alpha)$. Agora mostraremos a inclusão contrária. O grupo $\operatorname{Ker}(\alpha)$ é gerado pelos elementos $u_{i}, 1 \leq i \leq k$ e pela definição da função $\eta$ cada um desses elementos estão em $\operatorname{Ker}(\eta)$, segue que $\operatorname{Ker}(\alpha) \subset \operatorname{Ker}(\eta)$. Portanto, assim que $\operatorname{Ker}(\alpha)=\operatorname{Ker}(\eta)$. Concluímos assim a demonstração do lema.

\subsection{2}

\section{Fim da prova do Teorema de Effros-Handelman-Shen}

Finalmente, estamos prontos para concluir a demonstração do Teorema 13.1.7. Falta mostrar a volta do teorema.

Suponha que $\left(G, G^{+}\right)$satisfaz a hipótese do teorema. Como, por hipótese, $G$ é enumerável e $G^{+} \subset G$, temos que $G^{+}$também é enumerável. Consideramos uma enumeração

$$
G^{+} \backslash\{0\}=\left\{g_{0}, g_{1}, \ldots\right\} .
$$

Definiremos, indutivamente, sequências de conjuntos finitos $\left\{A_{n}\right\}_{n \geq 0}$, $\left\{B_{n}\right\}_{n \geq 0}$, tais que $B_{n-1} \subset A_{n}$ e sequências de homomorfismos positivos $\left\{\alpha_{n}\right\}_{n \geq 0},\left\{\beta_{n}\right\}_{n \geq 0},\left\{\eta_{n}\right\}_{n \geq 0}$ e $\left\{\theta_{n}\right\}_{n \geq 1}$ onde

$$
\begin{aligned}
\alpha_{n} & :\left(\mathbb{Z} A_{n}, \mathbb{Z} A_{n}^{+}\right) \rightarrow\left(G, G^{+}\right), \\
\beta_{n} & :\left(\mathbb{Z} B_{n}, \mathbb{Z} B_{n}^{+}\right) \rightarrow\left(G, G^{+}\right), \\
\eta_{n} & :\left(\mathbb{Z} A_{n}, \mathbb{Z} A_{n}^{+}\right) \rightarrow\left(\mathbb{Z} B_{n}, \mathbb{Z} B_{n}^{+}\right), \\
\theta_{n} & :\left(\mathbb{Z} B_{n-1}, \mathbb{Z} B_{n-1}^{+}\right) \rightarrow\left(\mathbb{Z} A_{n}, \mathbb{Z} A_{n}^{+}\right) .
\end{aligned}
$$

Tais que

$$
\beta_{n} \circ \eta_{n}=\alpha_{n-1} \quad \text { e } \quad \alpha_{n} \circ \theta_{n-1}=\beta_{n-1} .
$$


Considere uma sequência de elementos distintos $\left\{a_{n}\right\}_{n \geq 0}$ Definimos

$$
A_{0}=B_{0}:=\left\{a_{0}\right\}, \quad \eta_{0}=\text { id } \quad \text { e } \quad \alpha_{0}\left(i a_{0}\right)=\beta_{0}\left(i a_{0}\right)=i g_{0}, \quad i \in \mathbb{Z}
$$

Como $g_{0} \in G^{+}, \alpha_{0}$ e $\beta_{0}$ são positivas. Pela definição, $\alpha_{0}$ é uma função injetiva e portanto, $\operatorname{Ker}\left(\alpha_{0}\right)=\operatorname{Ker}\left(\eta_{0}\right)$. Além disso, $\beta_{0} \circ \eta_{0}=\alpha_{0}$.

Agora suponha que $A_{k}, B_{k}, \beta_{k}, \eta_{k}$ estão definidos para todo $0 \leq k \leq n$, tais que $B_{i-1} \subset A_{i}$ para todo $1 \leq 1 \leq i \leq k$. Também assuma que definimos as funções $\theta_{k}$ como a inclusão de $\mathbb{Z} B_{k-1}$ em $\mathbb{Z} A_{k}$ para todo $0 \leq k \leq n$. Definimos

$$
A_{n+1}:=\left\{a_{n+1}\right\} \cup B_{n}
$$

e a função $\theta_{n+1}$ como a inclusão de $\mathbb{Z} B_{n}$ em $\mathbb{Z} A_{n+1}$. Considere a função $\alpha_{n+1}:\left(\mathbb{Z} A_{n+1}, \mathbb{Z} A_{n+1}^{+}\right) \rightarrow\left(G, G^{+}\right)$, definida por

$$
\alpha_{n+1}\left(i a_{n+1}+\sum_{b \in B_{N}} i_{b} b\right)=i g_{n+1}+\sum_{b \in B_{n}} i_{b} \beta_{n}(b)
$$

Como $g_{n+1} \in G^{+}$e $\beta_{n}$ é um homomorfismo positivo, temos que $\alpha_{n+1}$ é um homomorfismo positivo. Usando o Teorema 13.1.13, obtemos um conjunto $B_{n+1}$ e homomorfismos positivos $\beta_{n+1}: \mathbb{Z} B_{n+1} \rightarrow G$ e $\eta_{n+1}: \mathbb{Z} A_{n+1} \rightarrow \mathbb{Z} B_{n+1}$ tais que $\beta_{n+1} \circ \eta_{n+1}=\beta_{n}$ e que $\operatorname{Ker}\left(\eta_{n+1}\right)=\operatorname{Ker}\left(\beta_{n}\right)$. Desta forma, temos o seguinte diagrama comutativo:

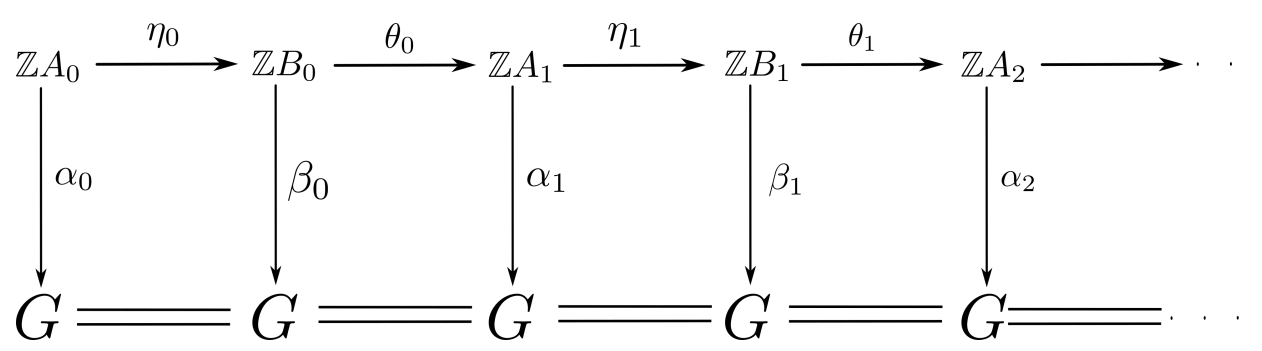

Figura 13.2: Diagrama comutativo

Para melhor compreensão, definimos as sequências auxiliares de conjuntos $\left\{C_{n}\right\}_{n \geq 0}$, onde $C_{2 n}=A_{n}$ e $C_{2 n+1}=B_{n}$ para todo $n \geq 0$ e de funções $\left\{f_{n}\right\}_{n \geq 0}$, onde $f_{2 n}=\eta_{n}$ e $f_{2 n+1}=\theta_{n}$. Obtemos que para todo $n \geq 1$ a função $f_{n}: \mathbb{Z} C_{n-1} \rightarrow \mathbb{Z} C_{n}$ é um homomorfismo positivo. Usando o Teorema 11.2.2, temos que existe um diagrama de Bratteli $\mathfrak{B}=(V, E, r, s)$, onde $V=\left\{C_{n}\right\}_{n \geq 0}$ e $h_{n}=f_{n}$ para todo $n \geq 0$.

Por definição de grupo de dimensão, o conjunto $\left(C_{\infty}, f_{\infty}\right)$ é o grupo de dimensão do diagrama $\mathfrak{B}$, que denotamos por $D(\mathfrak{B})$. Lembre que $D(\mathfrak{B})$ é 
formado por todas as classes de equivalência da relação dada por

$(a, n) \sim(b, m)$ se e só se existem $k, \ell \in \mathbb{Z}^{+}$tais que $f^{k}(a)=f^{\ell}(b)$.

Para finalizar a demonstração falta mostrar que $\left(G, G^{+}\right)$e $\left(D(\mathfrak{B}), D(\mathfrak{B})^{+}\right)$ são isomorfos.

Definimos a função $H: D(\mathfrak{B}) \rightarrow G$, por

$$
\begin{aligned}
H(\langle a, 2 n\rangle) & =\alpha_{n}(a), \quad \text { para } a \in \mathbb{Z} C_{2 n}=\mathbb{Z} A_{n}, \\
H(\langle b, 2 n+1\rangle) & =\beta_{n}(b), \quad \text { para } b \in \mathbb{Z} C_{2 n+1}=\mathbb{Z} B_{n} .
\end{aligned}
$$

Como para todo $n \geq 0, \alpha_{n}$ e $\beta_{n}$ são homomorfismos positivos, $H$ também é positivo.

Afirmação 13.1.21. Seja $a \in \mathbb{Z} A_{n}$ então

$$
\left.\langle a, 2 n\rangle=\rangle \eta_{n}(a), 2 n\right\rangle
$$

Prova. Por definição, $f_{n+1}=\eta_{n}$ e $\eta_{n}(a) \in \mathbb{Z} B_{n}$ então

$$
\left.\langle a, 2 n\rangle=\rangle \eta_{n}(a), 2 n\right\rangle
$$

Verificamos, então, que a afirmação é verdadeira

Afirmação 13.1.22. Seja $b \in \mathbb{Z} B_{n}$ então

$$
\left.\langle b, 2 n+1\rangle=\rangle \theta_{n}(b), 2 n+2\right\rangle .
$$

Prova. Como, por definição, $f_{n+1}=\theta_{n}$ e $\theta_{n}$ é a inclusão de $\mathbb{Z} B_{n}$ em $\mathbb{Z} A_{n+1}$, temos que $\theta_{n}(b) \in \mathbb{Z} A_{n+1}$ e

$$
\langle b, 2 n+1\rangle=\left\langle\theta_{n}(b), 2 n+2\right\rangle .
$$

Provando, assim, a afirmação.

Mostraremos agora que $H$ é bijetiva. Para a injetividade, veremos que $\operatorname{Ker}(H)=\{0\}$. Pela Afirmação 13.1.22, é suficiente considerar o caso em que para algum $a \in \mathbb{Z} A_{n}, H(\langle a, 2 n\rangle)=0$. Pela definição da função $H$, temos que

$$
H(\langle a, 2 n\rangle)=\alpha_{n}(a)
$$

$\log 0$

$$
a \in \operatorname{Ker}\left(\alpha_{n}\right)=\operatorname{Ker}\left(\eta_{n}\right)
$$


Então, pela Afirmação 13.1.21

$$
\langle a, 2 n\rangle=\left\langle\eta_{n}(a), 2 n+1\right\rangle=0 .
$$

Portanto, $H$ é injetiva.

Para mostrar que a função $H$ é sobrejetiva é suficiente mostrar que $G^{+} \subset$ $H\left(D(\mathfrak{B})^{+}\right)$, já que pela definição de cone positivo, temos que $G=G^{+}-G^{+}$. Considere $g \in G^{+}$, temos que $g$ é igual a 0 ou é igual a $g_{n}$, para algum $n \geq 0$. Se $g$ é igual a 0 , temos que $H(0)=g$. Caso $g \neq 0$, então

$$
g=g_{n}=\alpha_{n}\left(a_{n}\right)=h\left(\left\langle a_{n}, 2_{n}\right)\right\rangle .
$$

Como $a_{n} \in \mathbb{Z} A_{n}^{+}$, em qualquer um dos casos temos que $g$ é a imagem de algum elemento de $D(\mathfrak{B})^{+}$. Concluímos que $H$ é sobrejetiva, logo um isomorfismo.

Usando que $G^{+} \subset h\left(D(\mathfrak{B})^{+}\right)$e que $H$ é positivo, temos que $H$ é um isomorfismo ordenado. Portanto $\left(G, G^{+}\right)$e $\left(D(\mathfrak{B}), D(\mathfrak{B})^{+}\right)$são isomorfos. Concluímos, assim, a demonstração do Teorema de Effros-Handelman-Shen. 


\section{O Teorema de Bratteli-Elliot-Krieger}

Sejam $R$ e $Q$ relações de equivalência étale nos conjuntos de Cantor $X$ e $Y$, respectivamente. Pelo Teorema 10.2.2, temos que se $(X, R)$ e $(Y, Q)$ são equivalentes então os invariantes $D(X, R)$ e $D(Y, Q)$ são isomorfos. O Teorema de Bratteli-Elliot-Krieger determina quando, para relações de equivalência AF os invariantes são invariantes completos, isto é, $(X, R)$ e $(Y, Q)$ são equivalentes se, e só se, $D(X, R)$ e $D(Y, Q)$ são isomorfos.

\section{1}

\section{Teorema de Bratteli-Elliot-Krieger}

Antes de enunciarmos o Teorema de Bratteli-Elliot-Krieger precisamos definir um entrelaçamento entre dois diagramas de Bratteli.

De forma geral, um entrelaçamento entre os diagramas de Bratteli $\mathfrak{B}$ e $\mathfrak{T}$ é um diagrama de Bratteli $\mathcal{Q}$ que quando considerada a subsequência dos níveis pares se transforma no diagrama de Bratteli $\mathfrak{B}$ e quando considerada a subsequência dos níveis ímpares se transforma no diagrama de Bratteli $\mathfrak{T}$.

Definição 14.1.1. Seja $\mathfrak{B}=\left(V=\left\{V_{n}\right\}_{n \geq 0}, E=\left\{E_{n}\right\}_{n \geq 1}, r=r_{n n \geq 1}, s=\right.$ $\left.\left\{s_{n}\right\}_{n \geq 1}\right)$ um diagrama de Bratteli. Definimos $E_{m, n}\left(v_{i}^{n}, v_{j}^{m}\right)$ como o conjunto formado por todos os caminhos que ligam $v_{i}^{n}$ a $v_{j}^{m}$.

Definição 14.1.2 (Entrelaçamento). Considere dois diagramas de Bratteli $\mathfrak{B}=(V, E, r, s)$ e $\mathfrak{T}=(W, F, \bar{r}, \bar{s})$. Um entrelaçamento é formado por uma sequência crescente de inteiros $\left\{n_{m}\right\}_{m \geq 0}$ com $n_{0}=0$ e $n_{1}=1$, uma sequência de conjuntos finitos não vazios $\left\{A_{m}\right\}_{m \geq 1}$ e sequências de funções $\left\{p_{m}\right\}_{m \geq 1}$ e $\left\{q_{m}\right\}_{m \geq 1}$, tais que A coleção

$$
\left(Q=\left\{Q_{m}\right\}_{m \geq 0}, A=\left\{A_{m}\right\}_{m \geq 1}, p=\left\{p_{m}\right\}_{m \geq 1}, q=\left\{q_{m}\right\}_{m \geq 1}\right)
$$


forma um diagrama Bratteli, onde

$$
\begin{aligned}
& A_{1}=F_{1} \\
& Q_{m}=V_{n_{m}}, \quad \text { se m é par, } \\
& Q_{m}=W_{n_{m}}, \quad \text { se } m \text { é impar } \\
& p_{m}: A_{m} \rightarrow W_{n_{m-1}}, \quad q_{m}: A_{m} \rightarrow V_{n_{m}} \quad \text { se mé par, } \\
& p_{m}: A_{m} \rightarrow V_{n_{m-1}}, \quad q_{m}: A_{m} \rightarrow W_{n_{m}} \quad \text { se mé émpar },
\end{aligned}
$$

que verificam as seguintes condições:

1. Para cada $m \geq 0$ par e cada

$$
\left(v_{i}^{n_{m}}, v_{j}^{n_{m+2}}\right) \in V_{n_{m}} \times V_{n_{m+2}}
$$

existe uma bijeção entre os conjuntos $A_{m, m+2}\left(v_{i}^{n_{m}}, v_{j}^{n_{m+2}}\right) \quad e$ $E_{n_{m}, n_{m+2}}\left(v_{i}^{n_{m}}, v_{j}^{n_{m+2}}\right)$,

2. Para cada $m \geq 0$ impar e cada

$$
\left(w_{i}^{n_{m}}, w_{j}^{n_{m+2}}\right) \in W_{n_{m}} \times W_{n_{m+2}}
$$

existe uma bijeção entre os conjuntos $\quad A_{m, m+2}\left(w_{i}^{n_{m}}, w_{j}^{n_{m+2}}\right) \quad e$ $F_{n_{m}, n_{m+2}}\left(w_{i}^{n_{m}}, w_{j}^{n_{m+2}}\right)$.

Denotaremos um entrelaçamento entre dois diagramas de Bratteli $\mathfrak{B}$ e $\mathfrak{T}$ por $\mathfrak{Q}(\mathfrak{B}, \mathfrak{T})$.

Considere o diagrama de Bratteli $\mathfrak{E}$ apresentado na Figura 14.1.

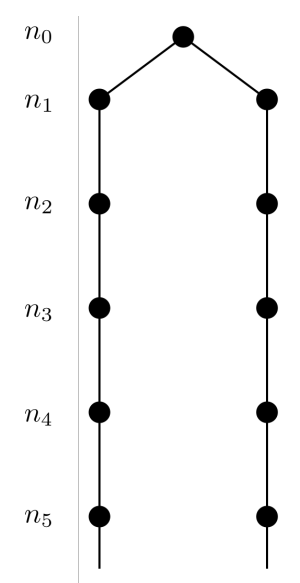

Figura 14.1: Diagrama de Bratteli E.

Temos que $\mathfrak{E}=\mathfrak{Q}(\mathfrak{E}, \mathfrak{E})$. Considere o diagrama de Bratteli gerado passando $\mathfrak{E}$ à subsequência dos níveis pares, que denotaremos por $\mathfrak{B}$ e o 
diagrama de Bratteli gerado passando $\mathfrak{E}$ passando a sequência dos níveis ímpares, que denotaremos por $\mathfrak{T}$, temos que $\mathfrak{B}=\mathfrak{T}=\mathfrak{E}$. A Figura 14.2 representa $\mathfrak{Q}(\mathfrak{B}, \mathfrak{T})$. O diagrama de Bratteli formado pelas arestas azuis é o diagrama $\mathfrak{B}$ e o formado pelas arestas vermelhas é o diagrama $\mathfrak{T}$.

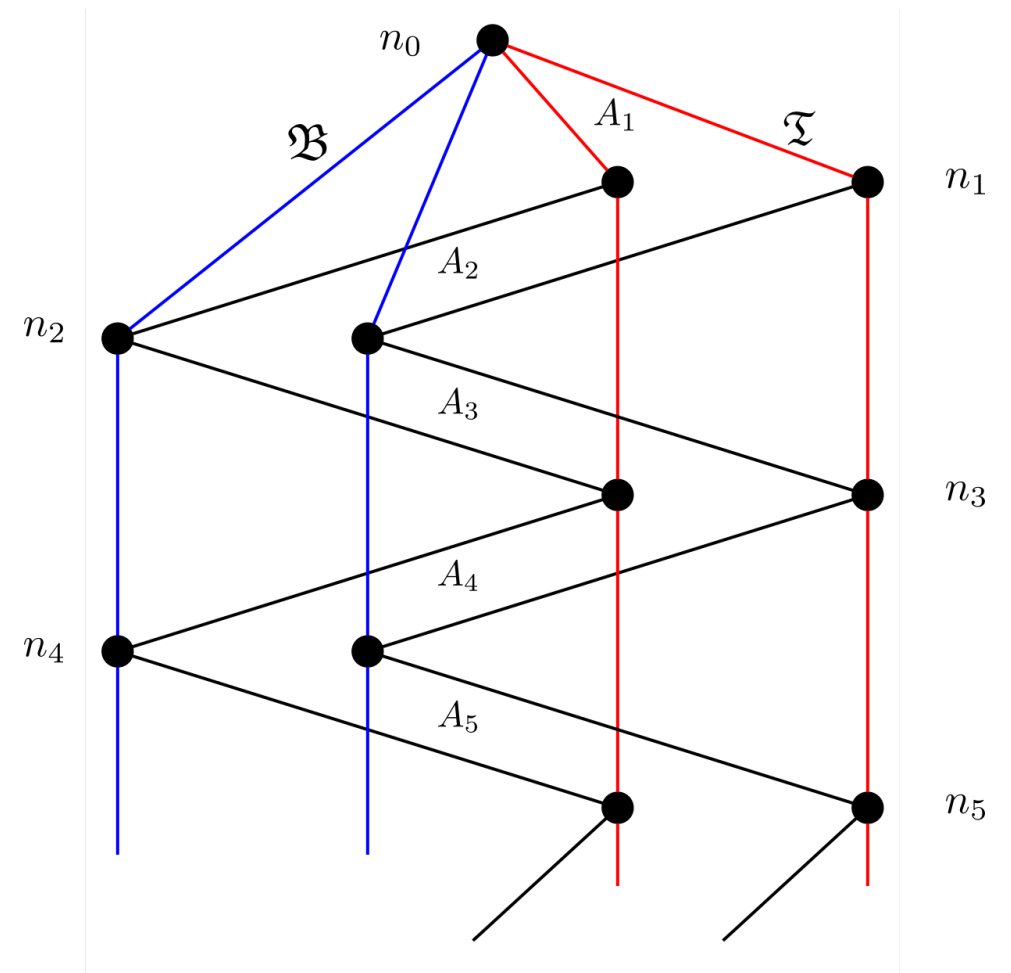

Figura 14.2: $\mathfrak{Q}(\mathfrak{B}, \mathfrak{T})$.

Iremos determinar quando existe um entrelaçamento entre dois diagramas de Bratteli.

Teorema 14.1.3 (Elliott-Bratteli-Krieger). Considere os diagramas de Bratteli $\mathfrak{B}=(V, E, r, s)$ e $\mathfrak{T}=(W, F, \bar{r}, \bar{s})$ e suas respectivas relações de equivalência AF, $\left(X_{\mathfrak{B}}, R_{\mathfrak{B}}\right)$ e $\left(X_{\mathfrak{T}}, R_{\mathfrak{T}}\right)$. As seguintes afirmações são equivalentes:

1. Existe um entrelaçamento entre $\mathfrak{B} e \mathfrak{T}$.

2. Existe um isomorfismo entre $\left(X_{\mathfrak{B}}, R_{\mathfrak{B}}\right)$ e $\left(X_{\mathfrak{T}}, R_{\mathfrak{T}}\right)$.

3. Existe um isomorfismo $\alpha$ ordenado entre os grupos de dimensão $D(\mathfrak{B}) e$ $D(\mathfrak{T})$ tal que $\alpha\left(\left\langle v_{0}, 0\right\rangle\right)=\left\langle w_{0}, 0\right\rangle$.

Prova. Mostraremos que as afirmações são equivalentes.

$(1) \Rightarrow(2)$ : Seja $\mathfrak{Q}$ um entrelaçamento entre $\mathfrak{B}$ e $\mathfrak{T}$. Portanto, $\mathfrak{B}$ e $\mathfrak{T}$ pode ser obtidos passando $\mathfrak{Q}$ a uma subsequência. Pelo Teorema 8.2.11, $\left(X_{\mathfrak{B}}, R_{\mathfrak{B}}\right) \cong\left(X_{\mathfrak{Q}}, R_{\mathfrak{Q}}\right)$ e $\left(X_{\mathfrak{T}}, R_{\mathfrak{T}}\right) \cong\left(X_{\mathfrak{Q}}, R_{\mathfrak{Q}}\right)$. Portanto $\left(X_{\mathfrak{B}}, R_{\mathfrak{B}}\right) \cong\left(X_{\mathfrak{T}}, R_{\mathfrak{T}}\right)$.

$(2) \Rightarrow(3)$ : Esta implicação é exatamente o conteúdo do Teorema 10.2.2. 
$(3) \Rightarrow(1)$ : Para produzir um entrelaçamento devemos definir uma sequência crescente de inteiros $\left\{n_{m}\right\}_{m \geq 0}$ e uma sequência de conjuntos $\left\{A_{m}\right\}_{m \geq 1}$, que será a coleção de arestas do diagrama de Bratteli do entrelaçamento, satisfazendo as propriedades da definição. Lembre que para $\ell<k$, a função $h_{\mathfrak{B}_{\ell, k}}: \mathbb{Z} V_{\ell} \rightarrow \mathbb{Z} V_{k}$ é definida por

$$
h_{\mathfrak{B}_{\ell, k}}=h_{\mathfrak{B}_{k}} \circ h_{\mathfrak{B}_{k-1}} \circ \cdots \circ h_{\mathfrak{B}_{m}} \circ h_{\mathfrak{B}_{m+1}}
$$

onde para cada $n \geq 1$ a função $h_{\mathfrak{B}_{n}}: \mathbb{Z} V_{n-1} \rightarrow \mathbb{Z} V_{n}$, é tal que

$$
h_{\mathfrak{B}_{n}}\left(v_{i}^{n-1}\right)=\sum_{j=1}^{\# V_{n}} \#\left(s^{-1}\left\{v_{i}^{n-1}\right\} \cap r^{-1}\left\{v_{j}^{n}\right\}\right) v_{j}^{n} .
$$

Por definição, a função $h_{\mathfrak{B}_{n_{m}, n_{m}+2}}$ determina o número de caminhos entre um vértice do nível $n_{m}$ e cada vértice no nível $n_{m+2}$. Analogamente, para a função $h_{\mathfrak{T}_{n_{m}, n_{m+2}}}$. Portanto, encontrar a sequência de conjuntos $\left\{A_{m}\right\}_{m \geq 0}$ satisfazendo os itens (1) e (2) da definição de entrelaçamento é o mesmo que demonstrar o seguinte resultado:

Proposição 14.1.4. Existe uma sequência crescente de números naturais $\left\{n_{m}\right\}_{m \geq 0}$ e uma sequência de homomorfismos positivos $\left\{f_{m}\right\}_{m \geq 1}$, onde

$$
\begin{aligned}
& f_{m}: \mathbb{Z} V_{n_{m-1}} \rightarrow \mathbb{Z} W_{n_{m}}, \quad \text { se } m \text { é ímpar }, \\
& f_{m}: \mathbb{Z} W_{n_{m-1}} \rightarrow \mathbb{Z} V_{n_{m}}, \quad \text { se } m \text { é par }
\end{aligned}
$$

tais que

- Se m é impar, se verifica que $f_{m} \circ f_{m-1}=h_{\mathfrak{T}_{n_{m-1}, n_{m}+1}}$ e que $\left\langle f_{m}(v), n_{m}\right\rangle=$ $\alpha\left(\left\langle v, n_{m}\right\rangle\right)$, para todo $v \in V_{n_{m-1}}$.

- Se m é par, se verifica que $f_{m} \circ f_{m-1}=h_{\mathfrak{B}_{n_{m-1}, n_{m+1}}}$ e que $\left\langle f_{m}(w), n_{m}\right\rangle=$ $\alpha^{-1}\left(\left\langle w, n_{m}\right\rangle\right)$, para todo $w \in W_{n_{m-1}}$.

Assuma que a proposição é verdadeira. Seja $m \geq 1$ e ímpar. Considere $v \in \mathbb{Z} V_{n_{m-1}}$, então $f_{m}(v) \in \mathbb{Z} W_{n_{m}}$. Podemos, então, escrever que

$$
f_{m}(v)=\sum_{w^{n_{m}} \in W_{n_{m}}}=a_{n_{m}} w^{n_{m}}, \quad a_{n_{m}} \geq 0
$$

Definimos, então que existem $a_{n_{m}}$ arestas ligando o vértice $v$ ao vértice $w^{n_{m}}$. Dessa forma, $f_{m} \circ f_{m-1}$ determina o número de arestas que ligam um vértice de $\mathbb{Z} W_{n_{m-1}}$ aos vértices de $\mathbb{Z} W_{n_{m+1}}$. Como pelo Lema 14.1.4,

$$
f_{m} \circ f_{m-1}=h_{\mathfrak{T}_{n_{m-1}, n_{m+1}}}
$$


a condição 2 é satisfeita. Se $m$ é par obteremos que a condição 1 é satisfeita.

Dito isto, provaremos a Proposição 14.1.4.

Prova. Construiremos as sequências indutivamente. Começamos definindo $n_{0}=0, n_{1}=1$ e $f_{1}=h_{\mathfrak{T}_{0}}$. Como $W_{0}=V_{0}$, temos que $f_{1}: \mathbb{Z} V_{0} \rightarrow \mathbb{Z} W_{1}$. Agora, suponha por indução que já construímos $n_{0}, n_{1}, \ldots, n_{m}$, e a sequência de homomorfismo positivos $f_{1}, f_{2}, \ldots, f_{m}$.

Suponha que $m$ é par. Encontraremos $n_{m+1}$ e o homomorfismo positivo $f_{m+1}$.

Lema 14.1.5. Existe $n>n_{m}$ tal que para cada $v \in \mathbb{Z} V_{n_{m}}$ existe $w_{v} \in \mathbb{Z} W_{n}$ que satisfaz

$$
\alpha(\langle v, m\rangle)=\left\langle w_{v}, n\right\rangle
$$

Prova. Considere $v$ um elemento de $V_{n_{m}}$, então $v \in \mathbb{Z} V_{n_{m}}^{+}$e, por definição, $\left\langle v, n_{m}\right\rangle \in D(\mathfrak{B})^{+}$. Como $\alpha$ é um homomorfismo positivo, temos que $\alpha\left(\left\langle v, n_{m}\right\rangle\right) \in D(\mathfrak{T})^{+}$. Então existem $a_{v}$ e $n_{v}$ tais que $a_{v} \in \mathbb{Z} W_{n_{v}}^{+}$e

$$
\alpha\left(\left\langle v, n_{m}\right\rangle\right)=\left\langle a_{v}, n_{v}\right\rangle
$$

Dentre todas as possibilidades escolhemos o par $\left(a_{v}, n_{v}\right)$ tal que $n_{v}$ seja mínimo. Definimos

$$
n:=\max \left\{n_{v}, n_{m} \mid v \in V_{n_{m}}\right\}+1 .
$$

Sabemos que, pela definição da relação de equivalência

$$
\left\langle a_{v}, n_{v}\right\rangle=\left\langle h_{n_{v}, n_{v}+k}\left(a_{v}\right), n_{v}+k\right\rangle \quad \text { para todo } k \geq 0 .
$$

Dessa forma, para todo $v \in \mathbb{Z} V_{n_{m}}$, existe $w_{v} \in \mathbb{Z} W_{n}$ tal quer $\alpha\left(\left\langle v, n_{m}\right\rangle\right)=$ $\left\langle w_{v}, n\right\rangle$. Concluímos, assim, a demonstração do lema.

Agora, considere a função $\bar{g}: V_{n_{m}} \rightarrow \mathbb{Z} W_{n}^{+}$, definida por

$$
\bar{g}(v):=h_{\mathfrak{T}_{n_{v}, n}}\left(a_{v}\right)=w_{v} \in \mathbb{Z} W_{n}^{+} .
$$

Podemos estender a função $\bar{g}$ de maneira única para um homomorfismo positivo $g: \mathbb{Z} V_{n_{m}} \rightarrow \mathbb{Z} W_{n}$.

Lema 14.1.6. Para todo $v \in V_{n_{m}}, \alpha\left(\left\langle v, n_{m}\right\rangle\right)=\langle g(v), n\rangle$. Além disso, para todo $k>n, \alpha\left(\left\langle v, n_{m}\right\rangle\right)=\left\langle h_{\mathfrak{T}_{n, k}} \circ g(v), n\right\rangle$.

Prova. Seja $v \in V_{n_{m}}$, então

$$
\alpha\left(\left\langle v, n_{m}\right\rangle\right)=\left\langle a_{v}, n_{v}\right\rangle=\left\langle h_{\mathfrak{T}_{n_{v}, n}}\left(a_{v}\right), n\right\rangle=\langle g(v), n\rangle .
$$


Além disso, seja $k>n$ e considere a composição $h_{\mathfrak{T}_{n, k}} \circ g$. Como

$$
h_{\mathfrak{T}_{n v}, k}=h_{\mathfrak{T}_{n, k}} \circ h_{\mathfrak{T}_{n_{v}, n}}=h_{\mathfrak{T}_{n_{v}, k}},
$$

Então,

$$
\begin{aligned}
\left\langle h_{\mathfrak{T}_{n, k}} \circ(v), k\right\rangle=\left\langle h_{\mathfrak{T}_{n, k}} \circ h_{\mathfrak{T}_{n v}, n}\left(a_{v}\right), k\right\rangle & =\left\langle h_{\mathfrak{T}_{n v}, n}\left(a_{v}\right), n\right\rangle \\
& =\alpha\left(\left\langle v, n_{m}\right\rangle .\right.
\end{aligned}
$$

Finalizamos, assim, a prova do lama

Gostaríamos de mostrar que dado $w \in W_{n_{m-1}}, g \circ f_{m}(w)=h_{\mathfrak{T}_{n_{m-1}, n}}(w)$. Porém, isto pode não ocorrer. O resultado válido é o seguinte:

Lema 14.1.7. Para cada $w \in \mathbb{Z} W_{n_{m-1}}$, temos que $\left\langle h_{\mathfrak{T}_{n_{m-1}, n}}(w), n\right\rangle=\langle g \circ$ $\left.f_{m}(w), n\right\rangle$.

Prova. Como $m$ é par e pela definição da função $g, f_{m}(w) \in \mathbb{Z} V_{n_{m}}$. Usando o

Lema 14.1.6 temos que

$$
\left\langle g\left(f_{m}(w)\right), n\right\rangle=\alpha\left(\left\langle f_{m}(w), n_{m}\right\rangle\right) .
$$

Pela hipótese de indução e como $m$ é par segue que

$$
\left\langle f_{m}(w), n\right\rangle=\alpha^{-1}\left(\left\langle w, n_{m-1}\right\rangle\right)
$$

Usando as equações (14.1.1) e (14.1.2), obtemos que

$$
\left\langle g\left(f_{m}(w)\right), n\right\rangle=\alpha\left(\alpha^{-1}\left(\left\langle w, n_{m-1}\right)\right)=\left\langle w, n_{m-1}\right\rangle .\right.
$$

Sabemos que, por definição,

$$
\left\langle w, n_{m-1}\right\rangle=\left\langle h_{\mathfrak{T}_{n_{m-1}, n}}(w), n\right\rangle
$$

Usando as equações (14.1.3) e (14.1.4), temos que

$$
\left\langle g\left(f_{m}(w)\right), n\right\rangle=\left\langle h_{\mathfrak{T}_{n_{m-1}, n}}(w), n\right\rangle .
$$

Finalizamos, assim, a demonstração do lema.

Pelo Lema 14.1.7, para cada $w \in \mathbb{Z} W_{n}$, os elementos $g \circ f_{m}(w)$ e $h_{\mathfrak{T}_{n_{m-1}, n}}(w)$ determinam o mesmo elemento em $D(\mathfrak{T})$. Então, para cada $w \in \mathbb{Z} W_{n}$, existe $\ell_{w}$ tal que

$$
h_{\mathfrak{T}_{n, \ell}}\left(g \circ f_{m}(w)\right)=h_{\mathfrak{T}_{n, \ell w}}\left(h_{\mathfrak{T}_{n_{m-1}, n}}(w)\right)=h_{\mathfrak{T}_{n_{m-1}, \ell w}}(w) .
$$


Escolheremos, para cada $w \in \mathbb{Z} W_{n}, \ell_{w}$ mínimo com essa propriedade. Definimos

$$
n_{m+1}=\max \left\{\ell_{w} \mid w \in W_{n_{m-1}}\right\}+1
$$

e

$$
f_{m+1}=h_{\mathfrak{T}_{n, n} m+1} \circ g .
$$

Pelo Lema 14.1.6, temos que para todo $v \in V_{n_{m+1}}$

$$
\left\langle f_{m+1}(v), n_{m+1}\right\rangle=\alpha\left(\left\langle v, n_{m}\right\rangle\right)
$$

Lema 14.1.8. $f_{m+1} \circ f_{m}=h_{\mathfrak{T}_{n_{m-2}, n_{m+1}}}$.

Prova. Seja $w \in W_{n-1}$, temos que

$$
\begin{aligned}
f_{m+1} \circ f_{m}(w) & =\left(h_{\mathfrak{T}_{n, n} m+1} \circ g\right) \circ f_{m}(w)=\left(h_{\mathfrak{T}_{\ell_{w}, n_{m+1}}} \circ h_{\mathfrak{T}_{n, \ell}}\right) \circ g \circ f_{m}(w) \\
& =h_{\mathfrak{T}_{\ell_{w}, n_{m+1}}} \circ h_{\mathfrak{T}_{n, \ell w}} \circ\left(g \circ f_{m}(w)\right)=h_{\mathfrak{T}_{\ell_{w}, n_{m+1}}} \circ h_{\mathfrak{T}_{n_{m}-1}, \ell_{w}}(w) \\
& =h_{\mathfrak{T}_{n_{m-1}, n_{m+1}}}(w)
\end{aligned}
$$

Provando, assim, o lema.

O caso em que $m$ é ímpar é feito de forma análoga. Concluímos, assim, a demonstração do lema.

Demonstramos, portanto, a existência de um entrelaçamento entre os diagramas $\mathfrak{B}$ e $\mathfrak{T}$. 


\section{5}

\section{Equivalência orbital forte}

Na hipótese do Teorema de Bratteli-Elliot-Krieger a relação de equivalência considerada é a relação $A F$. Pelo Teorema 7.1.13, dados um conjunto de Cantor $X$ e um homeomorfismo minimal $\varphi: X \rightarrow X$ podemos associar ao espaço $\left(X, R_{\varphi}\right)$ um diagrama de Bratteli $\mathfrak{B}$ tal que $X$ e $X_{\mathfrak{B}}$ são conjugados e as relações de equivalência $R_{\mathfrak{B}}$ e $R_{\varphi}$ estão relacionadas.

Lembre que dados dois espaços métricos $X$ e $Y$ e duas relações de equivalência $R$ e $Q$ orbitais em, respectivamente, $X$ e $Y$, dizemos que um homeomorfismo $h: X \rightarrow Y$ é uma equivalência orbital entre $R$ e $Q$ se, e só se, $h\left(\langle x\rangle_{R}\right)=\langle h(x)\rangle_{Q}$. Neste capítulo definiremos o que é uma equivalência orbital forte e classificaremos sistemas de Cantor minimais segundo equivalência orbital forte.

\section{1}

\section{Cociclos orbitais}

Proposição 15.1.1. Sejam dois conjuntos de Cantor $X$ e $Y$ e homeomorfismos $\varphi: X \rightarrow X$ e $\psi: Y \rightarrow Y$ sem pontos periódicos. Considere as relações orbitais $R_{\varphi}$ e $R_{\psi}$. Um homeomorfismo $h: X \rightarrow Y$ é uma equivalência orbital entre $R_{\varphi}$ e $R_{\psi}$ se e somente se existem funções $m_{h}: X \rightarrow \mathbb{Z}$ e $n_{h}: Y \rightarrow \mathbb{Z}$ tais que

$$
h \circ \varphi(x)=\psi^{m_{h}(x)} \circ h(x), \quad \text { para todo } x \in X
$$

$e$

$$
h^{-1} \circ \psi(y)=\varphi^{n_{h}(y)} \circ h^{-1}(y), \quad \text { para todo } y \in Y \text {. }
$$

As funções $m_{h}$ e $n_{h}$ são denominadas de cociclos orbitais. Repare que a continuidade dos cociclos orbitais não é exigida.

Prova. Suponha que $h: X \rightarrow Y$ é uma equivalência orbital. Como $x \sim_{R_{\varphi}} \varphi(x)$, temos que $h(x) \sim_{R_{\psi}} h(\varphi(x))$. Pela definição da relação $R_{\psi}$, existe um inteiro $k$ tal que

$$
\psi^{k}(h(x))=h(\varphi(x))
$$

Definimos $m_{h}(x)=\left\{k_{1}, \ldots, k_{\ell}\right\}$ como o conjunto formado dos inteiros que satisfazem a equação (15.1.1). Mostraremos que este conjunto contém um único elemento e o definiremos como $m_{h}(x)$. Suponha que $m_{h}(x)$ contém dois inteiros 
$k_{1}>k_{2}$ em $m_{h}(x)$. Então $\psi^{k_{1}}(h(x))=\psi^{k_{2}}(h(x))$. Assim,

$$
\psi^{k_{1}-k_{2}}(h(x))=h(x)
$$

Por hipótese, $\psi$ não possui pontos periódicos, então $k_{1}=k_{2}$ contradizendo a hipótese. Portanto, $m_{h}(x)$ contém um único elemento e podemos definir a função $m_{h}: X \rightarrow X$ que satisfaz

$$
h \circ \varphi(x)=\psi^{m_{h}(x)} \circ h(x) \quad \text { para todo } x \in X .
$$

Agora, suponha que $y \sim_{R_{\psi}} \psi(y)$, como $h$ é uma equivalência orbital, temos que $h^{-1}(y) \sim_{R_{\varphi}} h^{-1}(\psi(y))$. Pela definição da relação $R_{\varphi}$, existe um inteiro $\ell$ tal que

$$
\varphi^{\ell}\left(h^{-1}(y)\right)=h^{-1}(h(x)) .
$$

Definimos $n_{h}=\left\{\ell_{1}, \ell_{2}, \ldots, \ell_{k}\right\}$, que de forma análoga ao que fizemos anteriormente mostramos que possui um único elemento. Podemos, então, definir a função $n_{h}: Y \rightarrow \mathbb{Z}$ que satisfaz

$$
h^{-1} \circ \psi(y)=\varphi^{n_{h}(y)} \circ h^{-1}(y), \quad \text { para todo } y \in Y .
$$

Para a recíproca, mostraremos que se existem as funções $m_{h}: X \rightarrow \mathbb{Z}$ e $n_{h}: Y \rightarrow \mathbb{Z}$ como na hipótese, temos que $h\left(\langle x\rangle_{R_{\varphi}}\right)=\langle h(x)\rangle_{\varphi}$. Para isso, veremos que o primeiro conjunto está contido no segundo e vice-versa.

Lema 15.1.2. $h\left(\langle x\rangle_{R_{\varphi}}\right) \subset\langle h(x)\rangle_{R_{\psi}}$.

Prova. Seja $y \in h\left(\langle x\rangle_{R_{\varphi}}\right)$, então existe $z \in\langle x\rangle_{\varphi}$ tal que $h(z)=y$, e, portanto, $h^{-1}(y)=z$. Por definição de $R_{\varphi}$, existe inteiro $k$ tal que $\varphi^{k}(z)=x$. Então,

$$
\varphi^{k}(z)=\varphi^{k}\left(h^{-1}(y)\right)=x
$$

Por hipótese,

$$
\psi^{m_{h}(z)} \circ h(z)=h \circ \varphi(z)
$$

portanto,

$$
\psi^{m_{h}(z)}(y)=h \circ \varphi \circ h^{-1}(y) .
$$

Compondo a $k$ vezes a função $\psi^{m_{h}(z)}$, obtemos

$$
\left(\psi^{m_{h}(z)}\right)^{k}=h \circ \varphi^{k} \circ h^{-1} .
$$

Usando a equação (15.1.3), temos que

$$
\psi^{k m_{h}(z)}(y)=h \circ \varphi^{k} \circ h^{-1}(y)=h \circ \varphi^{k}(z)=h(x),
$$


portanto $y \in\langle h(x)\rangle_{R_{\psi}}$.

A demonstração de que $\langle h(x)\rangle_{R_{\psi}} \subset h\left(\langle x\rangle_{R_{\varphi}}\right)$ é análoga. Portanto, $h\left(\langle x\rangle_{R_{\varphi}}\right)=\langle h(x)\rangle_{R_{\psi}}$ para todo $x \in X$. Concluímos, então, que $h$ é uma equivalência orbital entre as relações $R_{\varphi}$ e $R_{\psi}$.

Lema 15.1.3. Sejam $X$ e $Y$ conjuntos de Cantor. Considere $\varphi: X \rightarrow X e$ $\psi: Y \rightarrow Y$ homeomorfismos minimais e $h: X \rightarrow Y$ uma equivalência orbital com cociclos orbitais $m_{h}: X \rightarrow \mathbb{Z}$ e $n_{n}: Y \rightarrow \mathbb{Z}$. As seguintes afirmações são verdadeiras.

1. Para todo $k \in \mathbb{Z}$, os conjuntos $m_{h}^{-1}(\{k\})$ e $n_{h}^{-1}(\{k\})$ são fechados.

2. Se $\left\{x_{i}\right\}_{i \geq 0}$ é uma sequência que converge para algum ponto $x \in X$, então o único ponto de acumulação finito da sequência $\left\{m_{h}\left(x_{i}\right)\right\}_{i \geq 0}$ é $m_{h}(x)$.

3. Se $\left\{y_{i}\right\}_{i \geq 0}$ é uma sequência que converge para algum ponto $y \in Y$, então o único ponto de acumulação finito da sequência $\left\{y_{h}\left(y_{i}\right)\right\}_{i \geq 0}$ é $m_{h}(y)$.

4. Se a função $m_{h}$ é descontínua em algum ponto $x \in X$, então $m_{h}$ é ilimitada em qualquer vizinhança de $x$.

5. Se a função $n_{h}$ é descontínua em algum ponto $y \in Y$, então $n_{h}$ é ilimitada em qualquer vizinhança de $y$.

Prova. As demonstrações serão realizadas para a função $m_{h}$. A prova para a função $n_{h}$ é análoga. Mostraremos, primeiro, que para todo $k \in \mathbb{Z}$, o conjunto $m_{h}^{-1}(\{k\})$ é fechado. Para isso, veremos que se $\left\{x_{i}\right\}_{i \geq 0}$ é uma sequência em $m_{h}^{-1}(\{k\})$ que converge para um ponto $x$, provaremos que $x \in m_{h}^{-1}(\{k\})$. Como para todo $i>0, x_{i} \in m_{k}^{-1}(\{k\})$, pela definição de cociclo orbital, temos, para todo $i \geq 0$, que

$$
h \circ \varphi\left(x_{i}\right)=\psi^{k} \circ h\left(x_{i}\right)
$$

Então

$$
\lim _{i \rightarrow \infty} h \circ \varphi\left(x_{i}\right)=\lim _{i \rightarrow \infty} \psi^{k} \circ h\left(x_{i}\right) .
$$

Por hipótese, as funções $h, \varphi$ e $\psi$ são contínuas, então

$$
h \circ \varphi(x)=\psi^{k} \circ h(x) .
$$

Portanto, $x \in m_{h}^{-1}(\{k\})$. Concluímos, então que que o conjunto $m_{h}^{-1}(\{k\})$ é fechado.

Agora, assuma que a função $m_{h}$ é descontínua em algum ponto $x \in X$. Então existe sequência $\left\{z_{n}\right\}_{n \geq 1}$ tal que $z_{n} \rightarrow x$ e $\left\{m_{h}\left(z_{n}\right)\right\}_{n \geq 1}$ não converge 
para $m_{h}(x)$. Considere o conjunto

$$
m_{h}\left(\left\{z_{n}\right\}_{n \geq 1}\right):=\left\{m_{h}\left(z_{n}\right) \mid n \in \mathbb{N}\right\}
$$

Se $m_{h}\left(\left\{z_{n}\right\}_{n \geq 1}\right)$ é um conjunto infinito, temos que toda vizinhança de $x$ é limitada. Agora, considere o caso em que

$$
m_{h}\left(\left\{z_{n}\right\}_{n \geq 1}\right)=\left\{L_{1}, \ldots, L_{j}\right\}
$$

Neste caso, existem $n_{0}$ e $1 \leq K \leq j$, tal que $z_{n}=L_{k}$ para todo $n \geq n_{0}$. Pelo item 1 o conjunto $m_{j}^{-1}\left(\left\{L_{K}\right\}\right)$ é fechado, então $x \in m_{j}^{-1}\left(\left\{L_{K}\right\}\right)$ e, portanto, $m_{h}\left(z_{n}\right) \rightarrow m_{h}(x)$, o que é uma contradição.

Teorema 15.1.4 (Boyle-Tomiyama). Sejam $X$ e $Y$ conjuntos de Cantor. Se $\varphi: X \rightarrow X$ e $\psi: Y \rightarrow Y$ são homeomorfismos minimais então as seguintes afirmações são equivalentes:

1. Existe uma equivalência orbital $h: X \rightarrow Y$ que possui cociclos contínuos.

2. Existe um isomorfismo $h:\left(X, R_{\varphi}\right) \rightarrow\left(Y, R_{\psi}\right)$.

3. $(X, \varphi)$ é topologicamente conjugado a $(Y, \psi)$ ou $\left(Y, \psi^{-1}\right)$.

Prova. Provaremos, somente, que $(1) \Leftrightarrow(2)$ e que $(3) \Rightarrow(2)$. Em [2] pode ser encontrada a demonstração completa do resultado.

$(1) \Rightarrow(2)$ : Suponha que os cociclos $m_{h}$ e $n_{h}$ são contínuos. Mostraremos, que $h \times h$ é um isomorfismo entre $R_{\varphi}$ e $R_{\psi}$. Para isto, provaremos que $h \times h$ é um homeomorfismo quando considerada a topologia étale.

Já que a função $m_{h}$ é contínua e o conjunto $\mathbb{Z}$ é discreto, para todo $x \in X$, existe um conjunto clopen $U_{x}$, onde a função $m_{h}$ é constante. Dado $n \in \mathbb{Z}$, temos que

$$
h \times h\left(U_{x}, \varphi^{n}\left(U_{x}\right)\right)=\left(h\left(U_{x}\right), h\left(\varphi^{n}\left(U_{x}\right)\right) .\right.
$$

Por hipótese, $m_{h}$ é constante em $U_{x}$, então existe $k \in \mathbb{Z}$ tal que $h\left(\varphi^{n}\left(U_{x}\right)\right)=$ $\psi^{k}\left(h\left(U_{x}\right)\right)$, então,

$$
h \times h\left(U_{x}, \varphi^{n}\left(U_{x}\right)\right)=\left(h\left(U_{x}\right), \psi^{k}\left(h\left(U_{x}\right)\right)\right) .
$$

Como a função $h$ é um homeomorfismo, o conjunto $\left(h\left(U_{x}\right), \psi^{k}\left(h\left(U_{x}\right)\right)\right.$ é um aberto de $R_{\psi}$. Provamos, assim, que $h^{-1} \times h^{-1}$ é contínua. Analogamente, como $n_{h}$ é contínua e $\mathbb{Z}$ é um conjunto discreto, para todo $y \in Y$ existe um conjunto clopen $V_{y}$, onde a função $n_{h}$ é constante. Assim, dado $m \in \mathbb{Z}$, existe um inteiro 
$\ell$ tal que $h^{-1}\left(\psi^{m}\left(V_{Y}\right)\right)=\varphi^{\ell}\left(h^{-1}\left(V_{Y}\right)\right)$. Portanto,

$$
h^{-1} \times h^{-1}\left(V_{y}, \psi^{m}\left(V_{Y}\right)\right)=\left(h^{-1}\left(V_{Y}\right), \varphi^{\ell}\left(h^{-1}\left(V_{y}\right)\right)\right) .
$$

Como $h$ é um homeomorfismo o conjunto $\left(h^{-1}\left(V_{Y}\right), \varphi^{\ell}\left(h^{-1}\left(V_{y}\right)\right)\right)$ é um aberto de $R_{\varphi}$. Concluímos, então que $h \times h$ e $h^{-1} \times h^{-1}$, além disto, como $h$ é um homeomorfismo e uma equivalência orbital, temos que $h \times h: R_{\varphi} \rightarrow R_{\psi}$ é uma bijeção. Portanto $h \times h: R_{\varphi} \rightarrow R_{\psi}$ é um homeomorfismo e portanto um isomorfismo entre $\left(X, R_{\varphi}\right)$ e $\left(Y, R_{\psi}\right)$.

$(2) \Rightarrow(1)$ : Suponha, agora, que existe um isomorfismo $h$ entre $\left(X, R_{\varphi}\right)$ e $\left(Y, R_{\psi}\right)$, isto é, $h: X \rightarrow Y$ é um homeomorfismo e $h \times h: R_{\varphi} \rightarrow R_{\psi}$ é um isomorfismo quando considerada a topologia étale.

Como $h \times h: R_{\varphi} \rightarrow R_{\psi}$ é um homeomorfismo, temos que $h$ é um equivalência orbital. Mostraremos que o cociclo $m_{h}$ é contínuo. A demonstração de que o cociclo $n_{h}$ é contínuo é análoga. Considere $x \in X$ e $U \subset X$ clopen que contém $x$. Como $h, \varphi$ e $\psi$ são homeomorfismos temos que os conjuntos $h(\varphi(U))$ e $\psi^{m_{h}(x)}(h(U))$ são abertos. Além disso,

$$
V_{m_{h}(x)}:=h(\varphi(U)) \bigcap \psi^{m_{h}(x)}(h(U)) \neq \emptyset .
$$

Portanto, o conjunto

$$
\varphi^{-1} \circ h^{-1}\left(V_{m_{h}(x)}\right) \text {. }
$$

é um aberto em que a função $m_{h}$ é limitada. Pelo Lema 15.1.3, a função $m_{h}$ é contínua.

$(3) \Rightarrow(2):$ suponha que $h: X \rightarrow Y$ é uma conjugação entre $(X, \varphi)$ e $(Y, \psi)$, mostraremos que $h$ e um isomorfismo entre $\left(X, R_{\varphi}\right)$ e $\left(Y, R_{\psi}\right)$. Seja $(x, z) \in R_{\varphi}$, existe $n \in \mathbb{Z}$ tal que $z=\varphi^{n}(x)$. Como $h$ é uma conjugação, temos que

$$
h(x)=h\left(\varphi^{n}(x)\right)=\psi^{n}(h(x)) .
$$

Então $(h(x), h(y)) \in R_{\psi}$. Agora, seja $(a, b) \in R_{\psi}$, temos que existe $k \in \mathbb{Z}$ tal que $b=\psi^{k}(a)$. Como $h$ é uma conjugação, segue que

$$
h^{-1}(b)=h^{-1}\left(\psi^{k}(a)\right)=\varphi^{k}\left(h^{-1}(a) .\right.
$$

Então, $\left(h^{-1}(a), h^{-1}(b)\right) \in R_{\varphi}$. Portanto, $h \times h\left(R_{\varphi}\right)=R_{\psi}$, em particular, $h \times h$ é uma sobrejeção. Como $h$ é injetiva, a função $h \times h$ também é injetiva. Falta mostrar que $h \times h$ e $h^{-1} \times h^{-1}$ são contínuas. Dado $U$ subconjunto clopen de 
$X$ e $n \in \mathbb{Z}$, considere o aberto $\left(U, \varphi^{n}(U)\right)$. Como $h$ é uma conjugação

$$
h \times h\left(U \cdot \varphi^{n}(U)\right)=\left(h(U), h\left(\varphi^{n}(U)\right)=\left(h(U), \psi^{n}(h(U))\right) .\right.
$$

Como $h$ é um homeomorfismo, o conjunto $h(U)$ é um subconjunto clopen de $Y$. Assim, a função $h^{-1} \times h^{-1}$ é contínua. A demonstração de que $h \times h$ é contínua é análoga. Concluímos que $h \times h: R_{\varphi} \rightarrow R_{\psi}$ é um homeomorfismo. Portanto $h$ é um isomorfismo entre $(X, \varphi)$ e $(Y, \psi)$.

$(3) \Rightarrow(1)$ : pelo mesmo argumento usado na implicação $(3) \Rightarrow(2)$, mostramos que $h: X \rightarrow Y$ é uma equivalência orbital. Precisamos mostrar que os cociclos são contínuos, No caso em que $h$ é uma conjugação topológica os cociclos são constantes, portanto são contínuos.

Concluímos a demonstração do teorema.

\section{2}

\section{Equivalência orbital forte e classificação}

Lembre, mais uma vez, que dados um espaço métrico $X$ e uma relação de equivalência $R$ em $X$, podemos considerar $R$ como o seguinte conjunto

$$
R=\left\{(x, y) \in X \times X \mid x \sim_{R} y\right\}
$$

Definição 15.2.1 (Equivalência orbital forte). Considere $X$ e $Y$ conjuntos de Cantor e $\varphi: X \rightarrow X$ e $\psi: Y \rightarrow Y$ homeomorfismos minimais. Uma equivalência orbital $h: X \rightarrow Y$ entre $R_{\varphi}$ e $R_{\psi}$ tal que os cociclos orbitais possuem cada um, no máximo, um ponto de descontinuidade, é dita forte.

Seja $\mathfrak{B}=(V, E, r, s)$ um diagrama de Bratteli. Lembre que na Seção 5.2 vimos que é possível obter um novo diagrama de Bratteli $\mathfrak{T}=(W, F, \overline{r, \bar{s}})$ a partir de $\mathfrak{B}$ através de uma subsequência. Além disso, definimos a função de blocos $f_{\mathfrak{B}, \mathfrak{T}}: X_{\mathfrak{B}} \rightarrow X_{\mathfrak{T}}$. Pela Proposição 5.2.2, $f_{\mathfrak{B}, \mathfrak{T}}$ é um homeomorfismo.

Pelo Teorema 5.3.5, se a relação de equivalência $R_{\mathfrak{B}}$ é minimal, então existe um diagrama de Bratteli $\mathfrak{T}=(W, F, \bar{r}, \bar{s})$, obtido através de $\mathfrak{B}$ a partir de uma subsequência, tal que $\mathfrak{T}$ possui todas as arestas conectadas. Isto é, para todo $n>0$, todo vértice de $W_{n}$ está conectado por meio de alguma aresta em $E_{n}$ com todo vértice de $W_{n-1}$.

Teorema 15.2.2. Seja $\mathfrak{B}=(V, E, r, s)$ uma diagrama de Bratteli. Suponha que os elementos $\mathbf{x}^{1}, \ldots, \mathbf{x}^{I}, \mathbf{y}^{1}, \ldots, \mathbf{y}^{I}$ estão em $X_{\mathfrak{B}}$ e que se considerarmos dois a dois distintos, eles não estão relacionados por $R_{\mathfrak{B}}$. Se $R_{\mathfrak{B}}$ é minimal, então existe um homeomorfismo $h: X_{\mathfrak{B}} \rightarrow X_{\mathfrak{B}}$ tal que: 
1. $h\left(\mathbf{x}^{\mathbf{i}}\right)=\mathbf{y}^{\mathbf{i}}$, para todo $1 \leq i \leq I$.

2. $h \times h: R_{\mathfrak{B}} \rightarrow R_{\mathfrak{B}}$ é um homeomorfismo quando considerada a topologia étale de $R_{\mathfrak{B}}$.

Prova. Precisaremos de um resultado preliminar.

Lema 15.2.3. Seja $\mathfrak{B}=(V, E, r, s)$ é um diagrama de Bratteli que possui todas as arestas conectadas. Se existem $\mathbf{x}^{1}, \ldots, \mathbf{x}^{I}, \mathbf{y}^{1}, \ldots, \mathbf{y}^{I}$ em $X_{\mathfrak{B}}$, tais que qualquer par formado por elementos desta lista não está em $R_{\mathfrak{B}}$, então é possivel obter a partir de $\mathfrak{B}$ através de uma subsequência um diagrama de Bratteli $\mathfrak{T}$ tal que para todo $n \geq 1$ existem pelo menos $3 I$ arestas distintas em $E_{n}$ e que as arestas entre vértices de níveis consecutivos nos elementos $f_{\mathfrak{B}, \mathfrak{T}}\left(\mathbf{x}^{1}\right), \ldots, f_{\mathfrak{B}, \mathfrak{T}}\left(\mathbf{x}^{I}\right), f_{\mathfrak{B}, \mathfrak{T}}\left(\mathbf{y}^{1}\right), \ldots, f_{\mathfrak{B}, \mathfrak{T}}\left(\mathbf{y}^{I}\right)$ são distintas.

Prova. Lembre que denotamos um elemento de $X_{\mathfrak{B}}$ como $\mathbf{x}=\left(x_{n}\right)_{n \geq 1}$. Faremos o caso $I=1$. Por hipótese, $\mathbf{x}^{1}=\left(x_{n}^{1}\right)_{n \geq 1}$ e $\mathbf{y}^{1}=\left(y_{n}^{1}\right)_{n \geq 1}$ não estão relacionados por $R_{\mathfrak{B}}$, então existe uma sequência crescente $\left\{\ell_{k}\right\}_{k \geq 1}$, tal que $x_{\ell_{k}}^{1} \neq y_{\ell_{k}}^{1}$. Definimos $n_{0}=0 \mathrm{e}$

$$
n_{1}=\min \left\{\ell_{k}>0 \mid \text { existe } e \in E_{\ell_{k}} \text { tal que } s(e)=s\left(x_{\ell_{k}}^{1}\right) \text { ou } s(e)=s\left(y_{\ell_{k}}^{1}\right)\right\} \text {. }
$$

Indutivamente, definimos

$$
n_{m}=\min \left\{\ell_{k}>n_{m-1} \mid \text { existe } e \in E_{\ell_{k}} \text { tal que } s(e)=s\left(x_{\ell_{k}}^{1}\right) \text { ou } s(e)=s\left(y_{\ell_{k}}^{1}\right)\right\} \text {. }
$$

A sequência $\left\{n_{m}\right\}_{m \geq 0}$ é crescente. Considere que $\mathfrak{T}=(W, F, \bar{r}, \bar{s})$ é obtido a partir de $\mathfrak{B}$ através da sequência $\left\{n_{m}\right\}_{m \geq 0}$.

Fixe $n \geq 0$. Por definição, $W_{n}=E_{n_{m-1}, n_{m}}$. Note que, a quantidade de arestas em $W_{n}$ é dada por

$$
\sum_{\substack{1 \leq i \leq \# V_{n_{m-1}} \\ 1 \leq j \leq \# v_{N_{m-1}+1}}}\left(\# E\left(v_{i}^{n_{m-1}}, v_{j}^{n_{m-1}+1}\right) \# s^{-1}\left(v_{j}^{n_{m-1}+1}\right) \prod_{n_{m-1}+2 \leq t \leq n_{m}} \# s^{-1}\left(v_{i_{t}}^{t}\right)\right)
$$

Por definição da sequência $\left\{n_{m}\right\}_{m \geq 0}$, existe $v_{i_{n_{m}-1}}^{n_{m}-1}$ tal que $\# s^{-1}\left(v_{i_{n_{m}-1}}^{n_{m}-1}\right)>1$, portanto, $\# W_{n}>\# E_{k}$ para todo $n_{m-1} \leq k \leq n_{m}$. Dessa forma, o diagrama obtido possui um número maior de arestas em cada nível do que $\mathfrak{B}$. Repetimos o processo acima quantas vezes forem necessárias até obter um diagrama de Bratteli que possui pelo menos $3 I$ arestas distintas em cada nível. Pela definição da subsequência $\left\{n_{m}\right\}_{m \geq 0}$ as arestas entre vértices de níveis consecutivos dos elementos $f_{\mathfrak{B}, \mathfrak{T}}\left(\mathbf{x}^{1}\right), \ldots, f_{\mathfrak{B}, \mathfrak{T}}\left(\mathbf{x}^{I}\right), f_{\mathfrak{B}, \mathfrak{T}}\left(\mathbf{y}^{1}\right), \ldots, f_{\mathfrak{B}, \mathfrak{T}}\left(\mathbf{y}^{I}\right)$ são distintas. Finalizamos, assim, a demonstração do lema. 
Por hipótese, a relação $R_{\mathfrak{B}}$ é minimal. Então é possível obter um novo diagrama de Bratteli $\mathfrak{T}$ através de $\mathfrak{B}$ a partir de uma subsequência, tal que $\mathfrak{T}$ possui todas as arestas conectadas e pelo menos $3 I$ arestas distintas em $E_{n}$ e que arestas entre vértices níveis consecutivos nos elementos $f_{\mathfrak{B}, \mathfrak{T}}\left(\mathbf{x}^{1}\right), \ldots, f_{\mathfrak{B}, \mathfrak{T}}\left(\mathbf{x}^{I}\right), f_{\mathfrak{B}, \mathfrak{T}}\left(\mathbf{y}^{1}\right), \ldots, f_{\mathfrak{B}, \mathfrak{T}}\left(\mathbf{y}^{I}\right)$ são distintas.

Suponha que existe um homeomorfismo $g: X_{\mathfrak{T}} \rightarrow X_{\mathfrak{T}}$ tal que

$-g\left(f_{\mathfrak{B}, \mathfrak{T}}\left(\mathbf{x}^{i}\right)\right)=\mathbf{y}^{i}$, para todo $1 \leq i \leq I$.

- $g \times g: R_{\mathfrak{T}} \rightarrow R_{\mathfrak{T}}$ é um homeomorfismo quando considerada a topologia étale de $R_{\mathfrak{T}}$.

Definimos a função

$$
h=f_{\mathfrak{B}, \mathfrak{T}}^{-1} \circ g \circ f_{\mathfrak{B}, \mathfrak{T}} .
$$

Temos que $h: X_{\mathfrak{B}} \rightarrow X_{\mathfrak{B}}$ é um homeomorfismo e satisfaz as condições do teorema. Faremos, então, o caso que $\mathfrak{B}$, possui todas as arestas conectadas, pelo menos $3 I$ arestas em $E_{n}$ e que as arestas entre vértices de níveis consecutivos nos elementos $\mathbf{x}^{1}, \ldots, \mathbf{x}^{I}, \mathbf{y}^{1}, \ldots, \mathbf{y}^{I}$ são distintas.

Começaremos escolhendo para cada $1 \leq i \leq I$ um elemento $\mathbf{z}^{i} \in X_{\mathfrak{B}}$. Já que $\mathfrak{B}$ possui todas as arestas conectadas é possível fazer a seguinte escolha de arestas:

- Para todo $1 \leq i \leq I, z_{1}^{i}=y_{1}^{i}$.

- Se $n \geq 2$ e par, escolhemos para cada $1 \leq i \leq I$, uma aresta $z_{n}^{i}$ distinta de $x_{n}^{i}$ e $y_{n}^{i}$, tal que

$$
s\left(z_{n}^{i}\right)=s\left(y_{n}^{i}\right) \quad \text { e } \quad r\left(z_{n}^{i}\right)=r\left(x_{n}^{i}\right) .
$$

- Para $n \geq 2$ ímpar, escolhemos para cada $1 \leq i \leq I$, uma aresta $z_{n}^{i}$ distinta de $x_{n}^{i}$ e $y_{n}^{i}$, tal que

$$
s\left(z_{n}^{i}\right)=s\left(x_{n}^{i}\right) \quad \text { e } \quad r\left(z_{n}^{i}\right)=r\left(y_{n}^{i}\right)
$$

Assim, para todo $1 \leq i \leq I, \mathbf{z}^{i}$ é um elemento em $X_{\mathfrak{B}}$.

Para cada $n \geq 1$ ímpar, considere a permutação $h_{0}$ em $E_{n-1, n+1}$ que permuta os pares de arestas $\left(x_{n}^{i}, x_{n+1}^{i}\right)$ e $\left(z_{n}^{i}, z_{n+1}^{i}\right)$ e não altera os outros pares. Para estender o domínio de $h_{0}$ para $X_{\mathfrak{B}}$, definimos

$$
h_{0}\left(e_{1}, e_{2}, \ldots\right)=\left(h_{0}\left(e_{1}, e_{2}\right), h_{0}\left(e_{3}, e_{4}\right), \ldots\right) \text {. }
$$


Para cada $n \geq 1$ par, considere a permutação $h_{1}$ em $E_{n-1, n+1}$ que permuta os pares $\left(z_{n}^{i}, z_{n+1}^{i}\right)$ e $\left(y_{n}^{i}, y_{n+1}^{i}\right)$ e não altera os outros pares. Estendemos o domínio de $h_{1}$ para $X_{\mathfrak{B}}$ de forma análoga ao que fizemos para $h_{0}$.

Repare que as funções $h_{0}$ e $h_{1}$ trocam um par de arestas em dois níveis consecutivos ou não o altera. Assim, para $k=0,1$,

$$
h_{k} \circ h_{k}(\mathbf{x})=\mathbf{x} .
$$

Portanto, a função inversa de $h_{k}$ é ela mesma e $h_{k}$ é uma bijeção.

Lema 15.2.4. Para $k=0,1$, a função $h_{k}: X_{\mathfrak{B}} \rightarrow X_{\mathfrak{B}}$ é contínua.

Prova. Sejam $n \geq 1$ e $p \in E_{0, n}$. Na Definição 5.1 .4 definimos um cilindro $C(p)$ de tamanho $n$ centrado em $p=\left(e_{1}, \ldots, e_{n}\right)$. Pela Proposição 5.1.5 os cilindros de tamanho par formam uma base da topologia de $X_{\mathfrak{B}}$. Fixe $k$ e $n \geq 1$ par. Mostraremos que $h_{k}^{-1}(C(p))$ é um aberto em $X_{\mathfrak{B}}$. Temos que

$$
h_{k}^{-1}(C(p))=h_{k}\left(C\left(\left(e_{1}, e_{2}, \ldots, e_{n}\right)\right)\right)=C\left(h_{k}\left(e_{1}, e_{2}\right), \ldots, h_{k}\left(e_{n-1}, e_{n}\right)\right)
$$

Portanto, a função $h_{k}$ é contínua.

Como $k=0,1$ a função $h_{k}$ é uma bijeção contínua e que $h_{k}^{-1}=h_{k}$. Portanto $h_{k}$ é um homeomorfismo. Definimos o homeomorfismo

$$
h:=h_{1} \circ h_{0} .
$$

Mostraremos agora que a função $h$ satisfaz as condições do teorema.

Fixe $1 \leq i \leq I$. Por definição da função $h$,

$$
\begin{aligned}
h\left(\mathbf{x}^{\mathbf{i}}\right) & =h_{1}\left(h_{0}\left(x_{1}^{i}, x_{2}^{i}, x_{3}^{i} \ldots\right)\right)=h_{1}\left(z_{1}^{i}, z_{2}^{i}, z_{3}^{i}, \ldots\right) \\
& =\left(z_{1}^{i}, y_{2}^{i}, y_{3}^{i}, \ldots\right)=\left(y_{1}^{i}, y_{2}^{i}, y_{3}^{i} \ldots\right)=\mathbf{y}^{i}
\end{aligned}
$$

Assim, a primeira condição do teorema é satisfeita.

Para mostrar que $h \times h: R_{\mathfrak{B}} \rightarrow R_{\mathfrak{B}}$ é um homeomorfismo quando considerada a topologia étale de $R_{\mathfrak{B}}$ precisamos mostrar que $h \times h\left(R_{\mathfrak{B}}\right)=R_{\mathfrak{B}}$, $h \times h$ é uma bijeção e que $h \times h$ e $(h \times h)^{-1}$ são contínuas.

Mostraremos, primeiro, que $h \times h\left(R_{\mathfrak{B}}\right) \subset R_{\mathfrak{B}}$. Para isto, considere $(\mathbf{a}, \mathbf{b}) \in h \times h\left(R_{\mathfrak{B}}\right)$. Existe $(\mathbf{x}, \mathbf{y}) \in R_{\mathfrak{B}}$ tal que $h(\mathbf{x})=\mathbf{a}$ e $h(\mathbf{y})=\mathbf{b}$. Como $(\mathbf{x}, \mathbf{y}) \in R_{\mathfrak{B}}$, existe $N>0$ tal que $x_{n}=y_{n}$ para todo $n \geq N$. Pela definição da função $h$,

$$
a_{n}=(h(\mathbf{x}))_{n}=(h(\mathbf{y}))_{n}=b_{n} \quad \text { para todo } n \geq N+1 .
$$


Então, $(\mathbf{a}, \mathbf{b}) \in R_{\mathfrak{B}}$.

Para provar a inclusão contrária, considere $(\mathbf{a}, \mathbf{b}) \in R_{\mathfrak{B}}$. Já que $h$ é sobrejetora, existem $\mathbf{x}$ e $\mathbf{y}$ em $X_{\mathfrak{B}}$, tais que $h(\mathbf{x})=\mathbf{a}$ e $h(\mathbf{y})=\mathbf{b}$. Como $(\mathbf{a}, \mathbf{b}) \in R_{\mathfrak{B}}$, existe $N>0$ tal que $a_{n}=b_{n}$ para todo $n \geq N$. Pela definição da função $h, x_{n}=y_{n}$ para todo $n \geq N$. Portanto, $(\mathbf{x}, \mathbf{y}) \in R_{\mathfrak{B}}$.

Provamos, então, que $h \times h: R_{\mathfrak{B}} \rightarrow R_{\mathfrak{B}}$ é uma função sobrejetora. Além disso, como $h$ é uma função injetora, temos que $h \times h$ também é. Portanto, $h \times h$ é uma bijeção.

Falta mostrar que $h \times h$ e $(h \times h)^{-1}$ são contínuas. Como $(h \times h)^{-1}=h \times h$, é suficiente provar que $(h \times h)^{-1}$ é continua. Lembre que na Seção 8.1 vimos que uma base da topologia étale de $R_{\mathfrak{B}}$ é formada por conjuntos da forma

$$
\gamma(p, q)=\left\{(\mathbf{x}, \mathbf{y}) \mid \mathbf{x} \in C(p), \mathbf{y} \in C(q) \text { e } x_{n}=y_{n} \text { para todo } n>N\right\}
$$

onde $p, q \in E_{0, N}$. Pela definição da função $h$, se $\mathbf{x} \in C(p)$, então $h(\mathbf{x}) \in C(h(p))$. Além disso, temos que $(h(\mathbf{x}))_{n}=(h(\mathbf{y}))_{n}$ para todo $n>N$. Portanto,

$$
h \times h(\gamma(p, q))=\left\{(\mathbf{x}, \mathbf{y}) \mid \mathbf{x} \in C(h(p)), \mathbf{y} \in C(h(q)) \text { e } x_{n}=y_{n} \forall n>N\right\},
$$

que é um aberto em $R_{\mathfrak{B}}$. Portanto $h \times h$ e $(h \times h)^{-1}$ são contínuas, e, portanto, homeomorfismos. Concluímos, assim, a demonstração do teorema.

Teorema 15.2.5. Considere conjuntos de Cantor $X$ e $Y$ e homeomorfismo minimais $\varphi: X \rightarrow X$ e $\psi: Y \rightarrow Y$. Existe uma equivalência orbital forte entre $(X, \varphi)$ e $(Y, \psi)$ se, e só se, existe um isomorfismo ordenado que preserva ordem unitária entre os invariantes $D\left(X, R_{\varphi}\right)$ e $D\left(Y, R_{\psi}\right)$.

Prova. Suponha que existe uma equivalência orbital forte $h: X \rightarrow Y$ com cociclos orbitais associados os cociclos $m$ e $n$, isto é,

$$
h \circ \varphi(x)=\psi^{m(x)} \circ h(x), \quad \text { para todo } x \in X
$$

e

$$
h^{-1} \circ \psi(y)=\varphi^{n(y)} \circ h^{-1}(y), \quad \text { para todo } y \in Y \text {. }
$$

Há dois casos de acordo com a continuidade das funções $m$ e $n$. Consideraremos, primeiro, o caso em que os cociclos $m$ e $n$ são, ambos, contínuos. Neste caso, pelo Teorema $15.1 .4, h$ é um isomorfismo entre $\left(X, R_{\varphi}\right)$ e $\left(Y, R_{\psi}\right)$. Usando o Teorema 10.2.2, existe um isomorfismo ordenado que preserva ordem unitária entre $D\left(X, R_{\varphi}\right)$ e $D\left(Y, R_{\psi}\right)$. 
Agora, suponha que $x_{0}$ e $y_{0}$ são os únicos pontos de descontinuidade das funções $m$ e $n$. Pelo Teorema 7.1.13, existem um diagrama de Bratteli ordenado, simples e próprio $\mathfrak{B}=(V, E, r, s, \geq)$ e um homeomorfismo $g_{X}: X_{\mathfrak{B}} \rightarrow X$ tal que

$$
g_{X} \circ \varphi_{\mathfrak{B}}=\varphi \circ g_{X}
$$

onde $\varphi_{\mathfrak{B}}$ é a função de Bratteli-Vershik associada a $\mathfrak{B}$ e $g_{X}\left(\mathbf{x}_{\max }\right)=x_{0}$.

Analogamente, existem um diagrama de Bratteli ordenado, simples e próprio $\mathfrak{T}$ e um homeomorfismo $g_{Y}: X_{\mathfrak{T}} \rightarrow Y$ tal que

$$
g_{Y} \circ \psi_{\mathfrak{T}}=\psi \circ g_{Y},
$$

onde $\psi_{\mathfrak{T}}$ é a função de Bratteli-Vershik associada a $\mathfrak{T}$ e $g_{Y}\left(\mathbf{y}_{\max }\right)=y_{0}$.

Lema 15.2.6. A função

$$
H: X_{\mathfrak{B}} \rightarrow X_{\mathfrak{T}}, \quad H:=g_{Y}^{-1} \circ h \circ g_{X}
$$

é uma equivalência orbital entre as relações $R_{\varphi_{\mathfrak{B}}}$ e $R_{\psi_{\mathfrak{T}}}$ com cociclos orbitas $m \circ g_{X}$ e $n \circ g_{Y}$.

Prova. Seja $\mathbf{x} \in X_{\mathfrak{B}}$. Temos que

$$
\begin{aligned}
H \circ \varphi_{\mathfrak{B}}(\mathbf{x}) & =g_{Y}^{-1} \circ h \circ g_{X} \circ \varphi_{\mathfrak{B}}(\mathbf{x})=g_{Y}^{-1} \circ h \circ \varphi \circ g_{X}(\mathbf{x}) \\
& =g_{Y}^{-1} \circ \psi^{m\left(g_{X}(\mathbf{x})\right)} \circ h\left(g_{X}(\mathbf{x})\right)=\psi^{m\left(g_{X}(\mathbf{x})\right)} \circ g_{Y}^{-1} \circ h \circ g_{X}(\mathbf{x}) \\
& =\psi_{\mathfrak{T}}^{m\left(g_{X}(\mathbf{x})\right)} \circ H(\mathbf{x}) .
\end{aligned}
$$

Analogamente, mostramos que dado $\mathbf{y} \in X_{\mathfrak{T}}$

$$
H^{-1} \circ \psi_{\mathfrak{T}}(\mathbf{y})=\varphi_{\mathfrak{B}}^{n\left(g_{Y}(\mathbf{y})\right)} \circ H^{-1}(\mathbf{y})
$$

Pela proposição 15.1.1, a função $H$ é uma equivalência orbital entre as relações $R_{\varphi_{\mathfrak{B}}}$ e $R_{\psi_{\mathfrak{T}}}$ com cociclos orbitais associados $m \circ g_{X}$ e $n \circ g_{Y}$. Concluímos, assim, a demonstração do lema

Pelo Lema 12.2.4 temos que

$$
D\left(X, R_{\varphi}\right) \cong D\left(X_{\mathfrak{B}}, R_{\varphi_{\mathfrak{B}}}\right) \quad \text { e } \quad D\left(Y, R_{\psi}\right) \cong D\left(X_{\mathfrak{T}, R_{\psi_{\mathfrak{T}}}}\right)
$$

Mostraremos, então que

$$
D\left(X_{\mathfrak{B}}, R_{\varphi_{\mathfrak{B}}}\right) \cong D\left(X_{\mathfrak{T}, R_{\psi_{\mathfrak{T}}}}\right) .
$$


Proposição 15.2.7. Se $h: X_{\mathfrak{B}} \rightarrow X_{\mathfrak{T}}$ é uma equivalência orbital forte com $\mathbf{x}_{\max }$ e $\mathbf{y}_{\max }$ pontos de descontinuidade dos cociclos $m: X_{\mathfrak{B}} \rightarrow \mathbb{Z}$ e $n: X_{\mathfrak{T}} \rightarrow \mathbb{Z}$, respectivamente, então

$$
D\left(X_{\mathfrak{B}}, R_{\varphi_{\mathfrak{B}}}\right) \cong D\left(X_{\mathfrak{T}, R_{\psi_{\mathfrak{T}}}}\right)
$$

Prova. Precisaremos de alguns resultados.

Lema 15.2.8. Para todo $\mathrm{x} \in X_{\mathfrak{B}}$ e $k \geq 1$,

$$
h\left(\varphi_{\mathfrak{B}}^{k}(\mathbf{x})\right)=\psi_{\mathfrak{T}}^{m(\mathbf{x})+m\left(\varphi_{\mathfrak{B}}(\mathbf{x})\right)+\cdots+m\left(\varphi_{\mathfrak{B}}^{k-1}(\mathbf{x})\right)}(h(\mathbf{x}))
$$

Prova. A demonstração será por indução. O caso $k=1$ já foi provado na Proposição 15.1.1. Considere que afirmação seja verdadeira para $k=\ell$. Mostraremos que também é verdadeira para $k=\ell+1$. Temos que

$$
h\left(\varphi_{\mathfrak{B}}^{\ell+1}(\mathbf{x})\right)=\psi_{\mathfrak{T}}^{m\left(\varphi_{\mathfrak{B}}^{\ell}(\mathbf{x})\right)}\left(h\left(\varphi_{\mathfrak{B}}^{\ell}(\mathbf{x})\right)\right)
$$

Pela hipótese de indução,

$$
h\left(\varphi_{\mathfrak{B}}^{\ell}(\mathbf{x})\right)=\psi_{\mathfrak{T}}^{m(\mathbf{x})+\cdots+m\left(\varphi_{\mathfrak{B}}^{\ell-1}(\mathbf{x})\right)}(h(\mathbf{x}))
$$

Então,

$$
\begin{aligned}
h\left(\varphi_{\mathfrak{B}}^{\ell+1}(\mathbf{x})\right) & =\psi_{\mathfrak{T}}^{m\left(\varphi_{\mathfrak{B}}^{\ell}(\mathbf{x})\right)}\left(\psi_{\mathfrak{T}}^{m(\mathbf{x})+\cdots+m\left(\varphi_{\mathfrak{B}}^{\ell-1}(\mathbf{x})\right)}(h(\mathbf{x}))\right) \\
& \left.=\psi_{\mathfrak{T}}^{\left.m(\mathbf{x})+\cdots+m\left(\varphi_{\mathfrak{B}}^{\ell-1}(\mathbf{x})\right)+m\left(\varphi_{\mathfrak{B}}^{\ell}(\mathbf{x})\right)\right)}(h(\mathbf{x}))\right) .
\end{aligned}
$$

Concluímos, assim, a demonstração do afirmação.

Considere os conjuntos

$$
R_{1}:=\left\{(\mathbf{x}, \mathbf{y}) \in R_{\varphi_{\mathfrak{B}}} \mid h \times h \text { é contínua em }(\mathbf{x}, \mathbf{y})\right\}
$$

e

$$
R_{2}:=\left\{(\mathbf{x}, \mathbf{y}) \in R_{\psi_{\mathfrak{\tau}}} \mid h^{-1} \times h^{-1} \text { é contínua em }(\mathbf{x}, \mathbf{y})\right\} .
$$

Lema 15.2.9. $R_{1}=R_{\mathfrak{B}}$ e $R_{2}=R_{\mathfrak{T}}$.

Prova. Mostraremos que $R_{1}=R_{\mathfrak{B}}$. Para isto, mostraremos que para cada $k \geq 1$, a função $h \times h$ é descontínua em $\left(\mathbf{x}, \varphi_{\mathfrak{B}}\right)$ se, só se, $\left(\mathbf{x}, \varphi_{\mathfrak{B}} \notin R_{\mathfrak{B}}\right)$. A demonstração de que $R_{2}=R_{\mathfrak{T}}$ é análoga.

Para cada $k \geq 1$, considere a função

$$
m_{k}: X \rightarrow \mathbb{Z}, \quad m_{k}(\mathbf{x})=m(\mathbf{x})+m\left(\varphi_{\mathfrak{B}}(\mathbf{x})\right)+\cdots+m\left(\varphi_{\mathfrak{B}}^{k-1}(\mathbf{x})\right)
$$


Já que a função $\varphi_{\mathfrak{B}}$ não possui pontos periódicos, $m_{k}$ é contínua se, e só se, $\mathbf{x}_{\max }$ não está no conjunto $\left\{\mathbf{x}, \varphi_{\mathfrak{B}}(\mathbf{x}), \ldots, \varphi_{\mathfrak{B}}^{k-1}(\mathbf{x})\right\}$. Pelo Lema 15.2.8, $h \times h$ é descontínua em $\left(\mathbf{x}, \varphi_{\mathfrak{B}}^{k}(\mathbf{x})\right)$ se, e só se,

$$
\mathbf{x}_{\max } \in\left\{\mathbf{x}, \varphi_{\mathfrak{B}}(\mathbf{x}), \ldots, \varphi_{\mathfrak{B}}^{k-1}(\mathbf{x})\right\}
$$

Isto significa que existe $1 \leq i \leq k-1$ tal que $\varphi_{\mathfrak{B}}^{i}(\mathbf{x})=\mathbf{x}_{\max }$. Logo, pela definição da função $\varphi_{\mathfrak{B}}$, temos que existe $\ell$ tal que $x_{j}=x_{\max _{j}}$ para todo $j \geq \ell$. Portanto

$$
\mathbf{x} \sim_{R_{\mathfrak{B}}} \varphi_{\mathfrak{B}}^{i}(\mathbf{x})=\mathbf{x}_{\max }
$$

Como $\varphi_{\mathfrak{B}}^{i}(\mathbf{x})=\mathbf{x}_{\max }$, temos que $\varphi_{\mathfrak{B}}^{i+1}(\mathbf{x})=\mathbf{x}_{\min }$. Com isso, usando que $k \geq i+1$, obtemos que $\left(\mathbf{x}_{\min }, \varphi_{\mathfrak{B}}^{k}(\mathbf{x})\right) \in R_{\mathfrak{B}}$. Portanto, $\left(\mathbf{x}, \varphi_{\mathfrak{B}}^{k}(\mathbf{x})\right)$ não está em $R_{\mathfrak{B}}$. Assim, $h \times h$ é descontínua em $\left(\mathbf{x}, \varphi_{\mathfrak{B}}^{k}(\mathbf{x})\right)$ se e somente se $\left(\mathbf{x}, \varphi_{\mathfrak{B}}^{k}(\mathbf{x})\right) \notin R_{\mathfrak{B}}$. Portanto, $R_{1}=R_{\mathfrak{B}}$.

Lema 15.2.10. $h \times h\left(R_{1}\right)=R_{2}$.

Prova. Suponha que $h \times h$ é contínua em algum ponto, de forma que também é contínua em alguma vizinhança $U$ desse ponto. Podemos supor que a vizinhança $U$ é compacta e aberta. Como $h \times h$ é continua em $U$, temos que $h \times h(U)$ é conjunto compacto e aberto. Assim, a função

$$
h \times h_{\mid U}: U \rightarrow h \times h(U)
$$

é uma bijeção contínua, de forma que $h \times h\left(R_{1}\right)=R_{2}$.

Pelos Lemas 15.2 .9 e 15.2.10, $h \times h$ determina um isomorfismo entre $\left(X, R_{\mathfrak{B}}\right)$ e $\left(Y, R_{\mathfrak{T}}\right)$. Pelo Teorema 10.2.2, $D\left(X_{\mathfrak{B}}, R_{\mathfrak{B}}\right)$ e $D\left(X_{\mathfrak{T}}, R_{\mathfrak{T}}\right)$ são isomorfos. Agora, pelo Lema 12.2.4, $D\left(X_{\mathfrak{B}}, R_{\mathfrak{B}}\right)$ é isomorfo a $D\left(X_{\mathfrak{B}}, R_{\varphi_{\mathfrak{B}}}\right)$ e $D\left(X_{\mathfrak{T}}, R_{\mathfrak{T}}\right)$ é isomorfo a $D\left(X_{\mathfrak{T}}, R_{\psi_{\mathfrak{T}}}\right)$. Então, $D\left(X_{\mathfrak{B}}, R_{\varphi_{\mathfrak{B}}}\right)$ e $D\left(X_{\mathfrak{T}}, R_{\psi_{\mathfrak{T}}}\right)$ são isomorfos. Concluímos, assim, a demonstração da proposição.

Finalizamos, então, a ida do teorema.

Agora, mostraremos a volta. Suponha que existe um isomorfismo ordenado entre $D\left(X, R_{\varphi}\right)$ e $D\left(X, R_{\psi}\right)$. Mais uma vez pelo Teorema 7.1.13, identificamos $\left(X, R_{\varphi}\right) \operatorname{com}\left(X_{\mathfrak{B}}, R_{\varphi_{\mathfrak{B}}}\right)$ e $\left(Y, R_{\psi}\right) \operatorname{com}\left(X_{\mathfrak{T}}, R_{\psi_{\mathfrak{T}}}\right)$, onde $\mathfrak{B}$ e $\mathfrak{T}$ são diagramas de Bratteli ordenados, simples e próprios. Como $\mathfrak{B}$ e $\mathfrak{T}$ são simples, as relações $R_{\mathfrak{B}}$ e $R_{\mathfrak{T}}$ são minimais. Considere que $\mathbf{x}_{\max }, \mathbf{x}_{\min }, \mathbf{y}_{\max }$ e $\mathbf{y}_{\min }$ são, respectivamente, os únicos caminhos maximais e minimais de $\mathfrak{B}$ e $\mathfrak{T}$. Pelo Lema 12.2.4, temos que

$$
D\left(X_{\mathfrak{B}}, R_{\mathfrak{B}}\right) \cong D\left(X_{\mathfrak{B}}, R_{\varphi_{\mathfrak{B}}}\right) \quad \text { e } \quad D\left(X_{\mathfrak{T}}, R_{\mathfrak{T}}\right) \cong D\left(X_{\mathfrak{T}}, R_{\psi_{\mathfrak{T}}}\right)
$$


Pelo Teorema de Bratteli-Elliot-Krieger, existe uma função $h: X_{\mathfrak{B}} \rightarrow X_{\mathfrak{T}}$ que determina um isomorfismo entre $\left(X_{\mathfrak{B}}, R_{\mathfrak{B}}\right)$ e $\left(X_{\mathfrak{T}}, R_{\mathfrak{T}}\right)$. Agora, considere os caminhos $\mathbf{x}^{1}=h\left(\mathbf{x}_{\max }\right), \mathbf{x}^{2}=h\left(\mathbf{x}_{\min }\right), \mathbf{y}^{1}=\mathbf{y}_{\min }$ e $\mathbf{y}^{2}=\mathbf{y}_{\min }$. Como a relação $R_{\mathfrak{T}}$ é minimal existe um homeomorfismo $\bar{h}: X_{\mathfrak{T}} \rightarrow X_{\mathfrak{T}}$ satisfazendo as condições do Teorema 15.2.2. Então a função $\bar{h} \circ h$ determina um isomorfismo entre $\left(X_{\mathfrak{B}}, R_{\mathfrak{B}}\right)$ e $\left(X_{\mathfrak{T}}, R_{\mathfrak{T}}\right)$ que leva $\mathbf{x}_{\max }$ em $\mathbf{y}_{\max }$ e $\mathbf{x}_{\min }$ e $\mathbf{y}_{\min }$ Pelo item 3 do Teorema 6.2.1, $\bar{h} \circ h$ é uma equivalência orbital entre $\left(X, R_{\varphi_{\mathfrak{B}}}\right)$ e $\left(X_{\mathfrak{T}}, R_{\psi_{\mathfrak{T}}}\right)$. Além disso, considere um caminho $\mathbf{x} \neq \mathbf{x}_{\max }$. Como $\varphi_{\mathfrak{B}}\left(\mathbf{x}_{\max }\right)=\mathbf{x}_{\min }$, temos que $\left(\mathbf{x}, \varphi_{\mathfrak{B}}(\mathbf{x})\right) \in R_{\mathfrak{B}}$, enquanto $\left(\mathbf{x}_{\max }, \varphi_{\mathfrak{B}}\left(\mathbf{x}_{\max }\right)\right) \notin R_{\mathfrak{B}}$. Como $\bar{h} \circ h$ é um isomorfismo entre $\left(X_{\mathfrak{B}}, R_{\mathfrak{B}}\right)$ e $\left(X_{\mathfrak{T}}, R_{\mathfrak{T}}\right)$, temos que

$$
(\bar{h} \circ h) \times(\bar{h} \circ h)\left(\mathbf{x}, \varphi_{\mathfrak{B}}(\mathbf{x})\right) \in R_{\mathfrak{T}} .
$$

Mas,

$$
(\bar{h} \circ h) \times(\bar{h} \circ h)\left(\mathbf{x}_{\max }, \mathbf{x}_{\min }\right)=\left(\bar{h} \circ h\left(\mathbf{x}_{\max }\right), \bar{h} \circ h\left(\mathbf{x}_{\min }\right)\right)=\left(\mathbf{y}_{\max }, \mathbf{y}_{\min }\right) \notin R_{\mathfrak{T}} .
$$

Portanto, a equivalência orbital $\bar{h} \circ h$ é contínua em todo ponto diferente de $\mathbf{x}_{\max }$. Pelo Teorema 15.1.4, $m$ é contínua em todo ponto distinto de $\mathbf{x}_{\max }$. Analogamente, mostramos que $n$ é contínua em todo ponto diferente de $\mathbf{y}_{\max }$. Concluímos, então, que $\bar{h} \circ h$ é uma equivalência orbital forte. 


\section{Equivalência orbital versus conjugação}

Sejam $X$ e $Y$ espaços métricos e $\varphi: X \rightarrow X$ e $\psi: Y \rightarrow Y$ homeomorfismos. Observamos que toda conjugação topológica entre $(X, \varphi)$ e $(Y, \psi)$ é, também, uma equivalência orbital. Provamos que se $X$ e $Y$ são espaços métricos compactos e conexos e os homeomorfismos $\varphi$ e $\psi$ não possuem pontos periódicos, então existe uma equivalência orbital entre $(X, \varphi)$ e $(Y, \psi)$ se, e só se, $(X, \varphi)$ é topologicamente conjugado a $(Y, \psi)$ ou a $\left(Y, \psi^{-1}\right)$.

Para o caso em que $X$ e $Y$ são conjuntos de Cantor e os homeomorfismos $\varphi$ e $\psi$ são minimais provamos que $(X, \varphi)$ é topologicamente conjugado com $(Y, \psi)$ ou com $\left(Y, \psi^{-1}\right)$ se, e só se, existe uma equivalência orbital entre $(X, \varphi)$ e $(Y, \psi)$ com cociclos contínuos.

Pode surgir a pergunta: há algum caso em que uma equivalência orbital não é uma conjugação? A resposta é sim. Apresentaremos um exemplo em que isto ocorre neste capítulo.

\section{1}

\section{Equivalência orbital versus conjugação}

Conceitos de teoria ergódica necessários podem ser encontrados em [7]. Aqui, apresentamos as definições mais importantes para o nosso objetivo.

Definição 16.1.1. Um sistema $(X, T)$ é dito unicamente ergódico se existe uma única medida $\mu$ invariante de probabilidade.

Os odômetros são exemplos de sistemas unicamente ergódicos.

No caso do espaço $\{0,1\}^{\mathbb{N}}$ os conjuntos mensuráveis são os cilindros. Dado $[\mathbf{x}]_{k}$ um cilindro de tamanho $k$ em $\{0,1\}^{\mathbb{N}}$, definimos a medida

$$
\mu\left([\mathbf{x}]_{k}\right)=\frac{1}{2^{k}} .
$$

A medida $\mu$ é a única medida invariante de probabilidade para o odômetro diádico.

Um homeomorfismo $f: \mathbf{S}^{1} \rightarrow \mathbf{S}^{1}$ que preserva orientação com número de rotação irracional, sem pontos periódicos e que não é topologicamente conjugado com alguma rotação irracional é chamado de homeomorfismo de Denjoy. Um sistema de Denjoy é um homeomorfismo de Denjoy restrito ao 
seu único conjunto de Cantor invariante. Mais sobre estes sistemas pode ser encontrado em [5].

Em [4] pode ser encontrada a demonstração do seguinte resultado:

Proposição 16.1.2. Se $(X, \varphi)$ é um sistema de Cantor unicamente ergódico, então existe uma equivalência orbital entre $(X, \varphi)$ e um sistema dinâmico formado por um odômetro ou entre $(X, \varphi)$ e um sistema de Denjoy.

Definição 16.1.3. Sejam $(X, \mathcal{S}, \mu)$ um espaço de probabilidade e $T$ uma transformação que preserva medida. T é fracamente misturadora se para todo par de conjuntos mensuráveis $A$ e $B$, existe $n \in \mathbb{Z}$ tal que

$$
\mu\left(T^{-n}(A) \bigcap A\right)>0 \quad \text { e } \quad \mu\left(T^{-n}(A) \bigcap B\right)>0 .
$$

O odômetros não são fracamente misturadores. Veremos para o odômetro diádico $\Phi$. Considere os conjuntos [0] e [1], temos que $\Phi([0])=[1]$ e $\Phi([1])=[0]$ e que $[0] \cap[1]=\emptyset$. Então, pra todo $n \in \mathbb{Z}$ um dos conjuntos $\Phi^{-n}([0]) \cap[0]$, $\Phi^{-n}([0]) \cap[1]$ é vazio e, portanto, possui medida nula.

Um exemplo de transformação fracamente misturada é a transformação de Chacón que foi criada por R.V. Chacón em 1969 [3]. Em [1] pode ser encontrada uma construção da transformação de Chacón. Aqui apresentaremos a primeira etapa da construção.

Primeiro, dividimos o intervalo $[0,1]$ nos intervalos disjuntos $\left[0, \frac{2}{3}\right)$ e $\left[\frac{2}{3}, 1\right]$. Dividimos o intervalo $\left[0, \frac{2}{3}\right)$ em três partes iguais e dividimos o intervalo $\left[\frac{2}{3}, 1\right]$ nos intervalos $\left[\frac{2}{3}, \frac{8}{9}\right)$ e $\left[\frac{8}{9}, 1\right]$. Este primeiro passo está ilustrado na Figura 16.1.

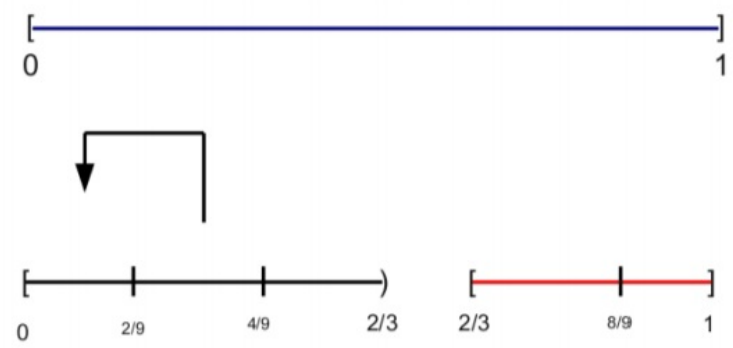

Figura 16.1: Primeiro passo da primeira etapa da Construção da transformação de Chacón.[1] 
Agora, faremos uma torre com os intervalos destacados como na Figura 16.2 .

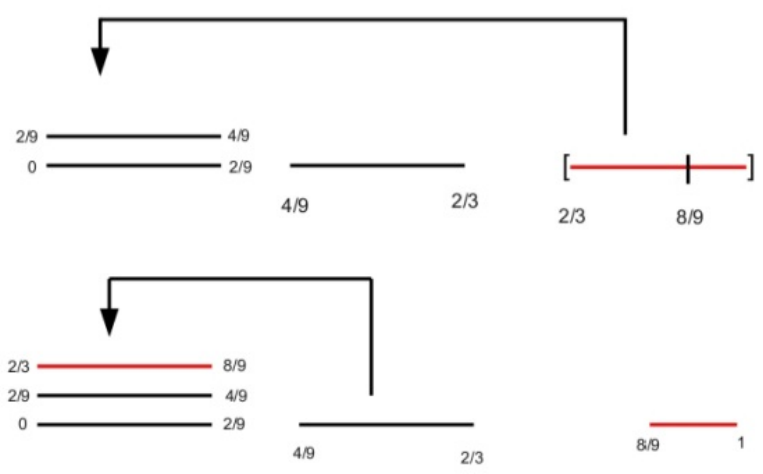

Figura 16.2: Segundo passo da primeira etapa da construção da transformação de Chacón.[1]

Ao final da primeira etapa temos uma torre de intervalos com quatro níveis e um intervalo separado. Na primeira etapa, definimos uma função que leva o primeiro andar torre para o andar seguinte e assim por diante, como ilustrado na Figura 16.3.
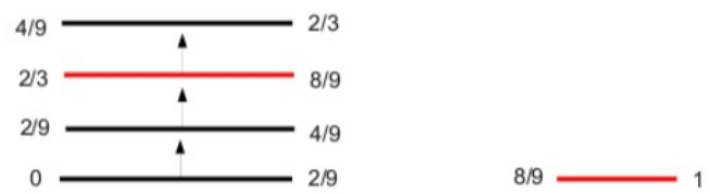

Figura 16.3: Fim da primeira etapa da construção da transformação de Chacón. $[1]$

A transformação de Chacón é um sistema de Cantor unicamente ergódico (veja [1]) e, portanto, existe uma equivalência orbital entre ela e um odômetro ou a um sistema de Denjoy. Neste caso, a equivalência orbital será com um odômetro, especificamente o odômetro definido em $\{0,1,2\}^{\mathbb{N}}$. Como, o odômetro não é fracamente misturador e a transformação de Chacón é, não existe uma conjugação entre a tranformação de Chacón e o odômetro. 


\section{Referências bibliográficas}

[1] AGYEMAN-PREMPEH, C.. Cutting and stacking in ergodic theory. Master's thesis, University of North Carolina at Chapel Hill, 2010.

[2] BOYLE, M.; TOMIYAMA, J.. Bounded topological orbit equivalence and $\mathbf{c}^{*}$-algebras. Journal of the Mathematical Society of Japan, 50(2):317329, 1998.

[3] CHACON, R. V.. Weakly mixing transformations which are not strongly mixing. Proceedings of the American Mathematical Society, 22(3):559-562, 1969.

[4] GIORDANO, T.; PUTNAM, I. F. ; SKAU, C. F.. Topological orbit equivalence and $c^{*}$-crossed products. J. Reine Angew. Math., 469:51111, 1995.

[5] HASSELBLATT, B.; KATOK, A.. Recurrence and equidistribuition on the circle. In: A First Course in Dynamics: with a Panorama of Recent Developments, chapter 4, p. 96-142. Cambridge University Press, 2014.

[6] PUTNAM, I. F.. Cantor minimal systems, volumen 70 de University Lecture Notes. American Mathematical Soc., 2018.

[7] SILVA, C. E.. Mixing notions. In: Invitation to Ergodic Theory, volumen 42 de Student Mathematical Library, chapter 6, p. 201-234. American Mathematical Soc., 2008. 